\title{
Estimating Solid-Liquid Phase Change Enthalpies and Entropies
}

\author{
James S. Chickos ${ }^{\text {a) }}$ \\ Department of Chemistry, University of Missouri-St. Louis, St. Louis Missouri 63121
}

William E. Acree, Jr.

Department of Chemistry, University of North Texas, Denton, Texas 76203

Joel F. Liebman

Department of Chemistry and Biochemistry, University of Maryland Baltimore County, Baltimore, Maryland 21250

Received January 7, 1999; revised manuscript received July 10, 1999

\begin{abstract}
A group additivity method based on molecular structure is described that can be used to estimate solid-liquid total phase change entropy $\left(\Delta_{0}^{T_{\text {fus }}} S_{\text {tpce }}\right)$ and enthalpy $\left(\Delta_{0}^{T_{\text {fus }}} H_{\text {tpce }}\right)$ of organic molecules. The estimation of these phase changes is described and numerous examples are provided to guide the user in evaluating these properties for a broad range of organic structures. A total of 1858 compounds were used in deriving the group values and these values are tested on a database of 260 additional compounds. The absolute average and relative errors between experimental and calculated values for these 1858 compounds are $9.9 \mathrm{~J} \cdot \mathrm{mol}^{-1} \cdot \mathrm{K}^{-1}$ and $3.52 \mathrm{~kJ} \cdot \mathrm{mol}^{-1}$, and 0.154 and 0.17 for $\Delta_{0}^{T_{\text {fus }}} S_{\text {tpce }}$ and $\Delta_{0}^{T_{\text {fus }}} H_{\text {tpce }}$, respectively. For the 260 test compounds, standard deviations of $\pm 13.0 \mathrm{~J} \cdot \mathrm{mol}^{-1} \cdot \mathrm{K}^{-1}\left(\Delta_{0}^{T_{\text {fus }}} S_{\text {tpce }}\right)$ and $\pm 4.88 \mathrm{~kJ} \mathrm{~mol}^{-1}\left(\Delta_{0}^{T_{\text {fus }}} H_{\text {tpce }}\right)$ between experimental and calculated values were obtained. Estimations are provided for both databases. Fusion enthalpies for some additional compounds not included in the statistics are also included in the tabulation. (C) 1999 American Institute of Physics and American Chemical Society. [S0047-2689(99)00106-3]
\end{abstract}

\section{Contents}

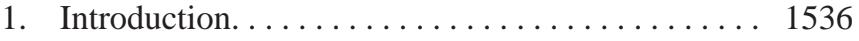

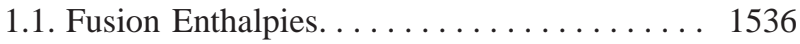

1.2. Fusion Entropies. . . . . . . . . . . 1536

2. Estimation of Total Phase Change Entropy

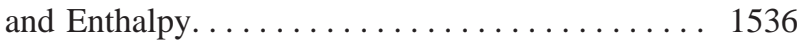

2.1. Derivation of Group Values. ........... 1536

3. Estimations of Hydrocarbons. . . . . . . . . . . . 1538

3.1. Acyclic and Aromatic Hydrocarbons....... 1538

3.1.1. Styrene. . . . . . . . . . . . . . . 1538

3.1.2. 1-Heptene. . . . . . . . . . . . . 1538

3.1.3. Perylene. . . . . . . . . . . . . . . . . . 1538

3.2. Nonaromatic Cyclic and Polycyclic

Hydrocarbons. . . . . . . . . . . . . . . . . . . . 1538

3.2.1. 10,10,13,13-Tetramethyl-1,5-

cyclohexadecadiyne. . . . . . . . 1539

3.2.2. Bullvalene. . . . . . . . . . . . . . 1539

3.2.3. Acenaphthylene. . . . . . . . . . . . 1539

4. Estimations of Hydrocarbon Derivatives....... . 1539

4.1. Acyclic and Aromatic Hydrocarbon

Derivatives.

${ }^{a)}$ Electronic mail: jscums1@jinx.ums1.edu

(C)1999 by the U.S. Secretary of Commerce on behalf of the United States. All rights reserved. This copyright is assigned to the American Institute of Physics and the American Chemical Society.

Reprints available from ACS; see Reprints List at back of issue.
4.1.1. Decachlorobiphenyl. . . . . . . . . . . 1540

4.1.2. N-acetyl-L-alanine amide. . . . . . . . 1540

4.1.3. Trifluoroacetonitrile. . . . . . . . . . . 1540

4.1.4. Isoquinoline. . . . . . . . . . . . 1540

4.2. Cyclic and Polycyclic Hydrocarbon

Derivatives... . . . . . . . . . . . . 1540

4.2.1. 2-Chlorodibenzodioxin. . . . . . . . . . 1540

4.2.2. 6,8,9-Trimethyladenine. . . . . . . . 1540

4.2.3. Lenacil. . . . . . . . . . . . . . . 1540

4.2.4. Cortisone. ................ 1541

4.3. Polymers. . . . . . . . . . . . . . . . . . . 1541

5. The Group Coefficient in Cycloalkyl Derivatives. 1541

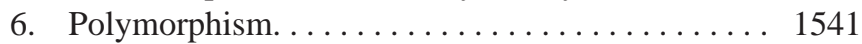

7. Statistics of the Correlation. . . . . . . . . . . . . 1542

7.1. Database Compounds. . . . . . . . . . . . . . . . 1542

7.2. Test Compounds. . . . . . . . . . . . . . . . . 1543

8. Acknowledgments. . . . . . . . . . . . . . . 1673

9. References...................... 1673

\section{List of Tables}

1. (a) Contributions of the hydrocarbon portion of acyclic and aromatic molecules............ 1543

1. (b) Contributions of the cyclic hydrocarbon portions of the molecule. . . . . . . . . . . . 1543

2. (a) Contributions of the functional group portion of the molecule................... 1544

2. (b) Contributions of functional groups as part of 
a ring. ......................... 1546

3. Estimations of total phase change entropies and enthalpies of hydrocarbons............... 1547

4. Estimations of total phase change entropies and enthalpies

A. Substituted aromatic and aliphatic molecules;

B. substituted cyclic molecules............... 1547

5. Experimental and calculated total phase change enthalpy and entropy of database............ 1548

6. Experimental and calculated total phase change enthalpies and entropies of fusion of polymers.... 1641

7. Calculated and experimental phase change enthalpies and entropies of test solids......... 1645

8. References to Tables 5, 6, and 7.......... 1668

\section{List of Figures}

1. Fusion entropy of the $n$-alkanes as a function of the number of methylene groups........... 1537

2. Total phase change entropies of the $n$-alkanes as a function of the number of methylene groups.... 1537

3. A comparison of calculated and experimental $\Delta_{0}^{T_{\text {fus }}} S_{\text {tpce }}$ of 1858 database compounds...........

4. A histogram illustrating the distribution of errors in estimating $\Delta_{0}^{T_{\text {fus }}} S_{\text {tpce }}$ of the database compounds........................

5. A comparison of calculated and experimental $\Delta_{0}^{T_{\text {fus }}} S_{\text {tpce }}$ of 260 test compounds..................

6. A histogram illustrating the distribution of errors in estimating $\Delta_{0}^{T_{\text {fus }}} S_{\text {tpce }}$ of 260 test compounds....

\section{Introduction}

\subsection{Fusion Enthalpies}

Fusion enthalpy is an important physical property of the solid state. The magnitude of the fusion enthalpy influences solute solubility in both an absolute sense and in its temperature dependence. This property plays an important factor in determining molecular packing in crystals and can be useful in correcting thermochemical data to a standard state when combined with other thermodynamic properties.

The discrepancy in numbers between the many new organic solids prepared and the few thermochemical measurements reported annually has encouraged the development of empirical relationships that can be used to estimate properties such as fusion enthalpy. We have found that techniques for estimating fusion enthalpies can play several useful roles. ${ }^{1-3}$ Perhaps most importantly, they provide a numerical value that can be used in cases when there are no experimental data. Estimations are also useful in selecting the most probable experimental value in cases where two or more values are in significant disagreement. Given the choice between an estimated or experimental value, selection of the experimental value is clearly preferable. However, large discrepancies between estimated and calculated values can also identify systems exhibiting dynamic or associative properties. Some molecular systems exhibit phase transitions that occur in the solid state that are related to the onset of mo- lecular motion. Others, such as liquid crystals exhibit nonisotropic molecular motion in the liquid phase. ${ }^{4}$ Both have associated with these phenomena, additional phase transitions that attenuate the enthalpy and entropy associated with fusion. A large positive discrepancy in the difference between estimated and experimentally measured fusion enthalpy is a good indication of this behavior.

\subsection{Fusion Entropies}

Very few general techniques have been developed for directly estimating fusion enthalpies, in part, as a consequence of the complex phase behavior exhibited by some compounds. Fusion enthalpies have been most frequently calculated from fusion entropies and the experimental melting temperature of the solid $T_{\text {fus }}$. One of the earliest estimation techniques is the use of Walden's Rule. ${ }^{5}$ The application of Walden's Rule provides a remarkably good approximation of $\Delta_{\text {fus }} H_{m}$, if one considers that the estimation is independent of molecular structure and based on only two parameters. Recent modifications of this rule have also been reported. ${ }^{6,7}$ Walden's Rule:

$$
\begin{aligned}
\Delta_{\text {fus }} H_{m}\left(T_{\text {fus }}\right) / T_{\text {fus }} & \approx 13 \mathrm{cal} \cdot \mathrm{K}^{-1} \cdot \mathrm{mol}^{-1} \\
& =54.4 \mathrm{~J} \mathrm{~mol}{ }^{-1} \mathrm{~K}^{-1} .
\end{aligned}
$$

A general method for estimating fusion entropies based on the principles of group additivity has been reported recently. ${ }^{8-10}$ This method has been developed from the assumption that unlike fusion enthalpy and entropy, the total phase change entropy associated in going from a rigid solid at $0 \mathrm{~K}$ to an isotropic liquid at the melting point, $T_{\text {fus }}$, is a group property and that this property can be estimated by standard group additivity techniques. The total phase change entropy has been defined as the sum of the entropy associated with all the phase changes occurring in the condensed phase prior to and including melting. The assumption that the total phase change entropy is a more reliable group property than fusion entropy is readily apparent from an examination of these two properties as a function of the number of methylene groups for the $n$-alkanes. This is illustrated in Figs. 1 and 2. Many alkanes have additional phase transitions with significant entropy components that influence the magnitude of the fusion entropy. This leads to the nonlinear behavior illustrated in Fig. 1. When these components are added together, the total phase change entropy shows a much better linear correlation. Some odd-even alternation as a function of the number of carbon atoms is evident similar to what is observed in the melting points of these compounds

\section{Estimation of Total Phase Change Entropy and Enthalpy}

\subsection{Derivation of Group Values}

Initial group values for a methyl and methylene group were derived from the intercept (one half the intercept) and 


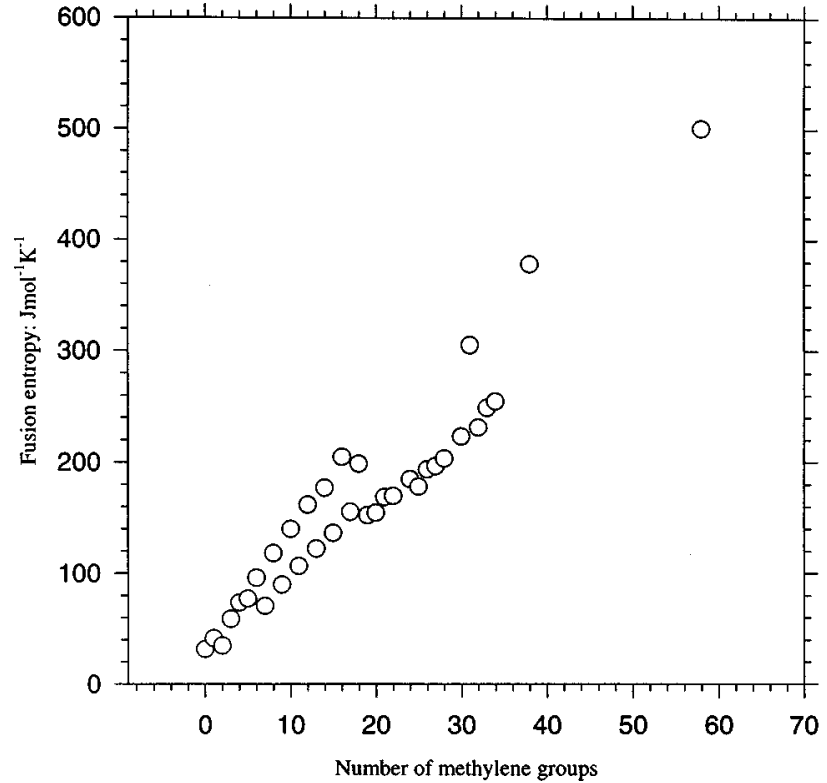

FIG. 1. Fusion entropy of the $n$-alkanes as a function of the number of methylene groups.

slope of the line of Fig. 2, respectively. Group values for carbon in other common environments were initially derived from experimental data of compounds with appropriate structures using these two group values. Subsequent refinements were possible as additional experimental data became available. Once values were assigned for most carbon groups, these values were allowed to vary until the value of the function:

$$
\sum_{i=1}^{n}\left[\Delta_{0}^{T_{\text {fus }}} S(\text { expt })-\Delta_{0}^{T_{\text {fus }}} S(\text { calcd })\right]^{2}
$$

did not change significantly upon successive iterations. Group values for the functional groups were derived in a similar fashion. Using group values for carbon established from the hydrocarbons, values for the functional groups in Tables 1 and 2 were derived. Once initial values for these groups were established, a similar least squares minimization of all the values were performed.

The total phase change entropy, $\Delta_{0}^{T_{\text {fus }}} S_{\text {tpce }}$, in most cases provides a good estimate of the entropy of fusion, $\Delta_{\text {fus }} S_{m}\left(T_{\text {fus }}\right)$. If there are no additional solid phase transitions then $\Delta_{0}^{T_{\text {fus }}} S_{\text {tpce }}$ becomes numerically equal to $\Delta_{\text {fus }} S_{m}\left(T_{\text {fus }}\right)$. From the experimental melting point and $\Delta_{\text {fus }} S_{m}\left(T_{\text {fus }}\right)$, it is possible to approximate the total phase change enthalpy, $\Delta_{0}^{T_{\text {fus }}} H_{\text {tpce }}$. Similarly, if there are no additional phase transitions then the total phase change enthalpy, $\Delta_{0}^{T_{\text {fus }}} H_{\text {tpce }}$, becomes numerically equivalent to the fusion enthalpy, $\Delta_{\text {fus }} H_{m}\left(T_{\text {fus }}\right)$.

A listing of the group parameters that can be used to estimate these phase change properties is presented in Tables 1 and 2. The group values in these tables have been updated from previous versions by the inclusion of new experimental data in the parameterizations. ${ }^{8,9}$ Before describing the appli-

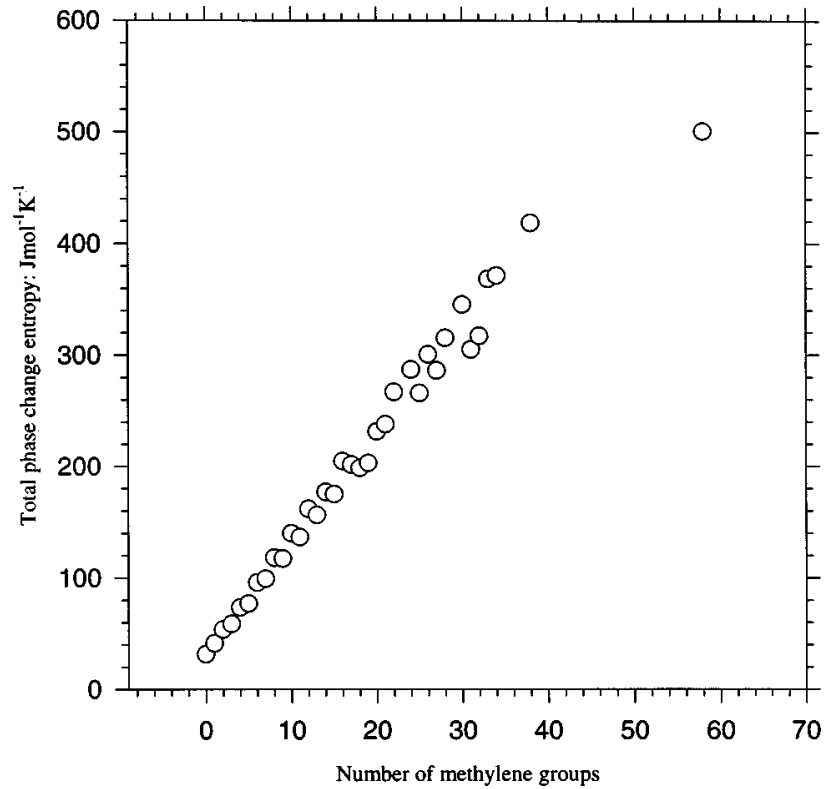

FIG. 2. Total phase change entropies of the $n$-alkanes as a function of the number of methylene groups.

cation of these parameters in the estimation of $\Delta_{0}^{T_{\text {fus }}} S_{\text {tpce }}$ and $\Delta_{0}^{T_{\text {fus }}} H_{\text {tpce }}$, the conventions used to describe these group values need to be defined. Primary, secondary, tertiary, and quaternary centers, as found on atoms of carbon, silicon, and their congeners, are defined solely on the basis of the number of hydrogens attached to the central atom, 3, 2, 1, 0, respectively. It should be noted that the experimental melting point along with an estimated value of $\Delta_{0}^{T_{\text {fus }}} S_{\text {tpce }}$ is necessary to estimate the fusion enthalpy of a compound. In addition, compounds whose liquid phase is not isotropic at the melting point are not modeled properly by these estimations. Those compounds forming liquid crystal or cholesteric phases as well amphiphilic compounds are currently overestimated by these parameters. A large discrepancy between the estimated total phase change enthalpy and experimental fusion enthalpy is a good indication of undetected solid-solid phase transitions or anisotropic liquid behavior.

The parameters used for estimating $\Delta_{0}^{T_{\text {fus }}} S_{\text {tpce }}$ of hydrocarbons and the hydrocarbon portions of more complex molecules are listed in Table 1 . The group value, $G_{i}$, associated with a molecular fragment is identified in the third column of the table. The group coefficients, $C_{i}$, are listed in column 4 of the table. These group coefficients are used to modify $G_{i}$ whenever a functional group is attached to the carbon in question. Functional groups are defined in Table 2. Group values reported in parenthesis are based on only a limited database (arbitrarily chosen as less then seven entries) and should be considered as tentative assignments. All values of $C_{i}$ and $C_{k}$ that are not specifically defined in Tables 1 and 2 are to be assumed equal to 1.0. The group coefficient for a methylene group in Table $1, C_{\mathrm{CH}_{2}}$, is applied differently from the rest and its application is discussed below. Introduction of this coefficient is new and differentiates this pro- 
tocol from earlier versions. The application of this group coefficient as well as the entire protocol is illustrated in the examples given in Tables 3 and 4 .

\section{Estimations of Hydrocarbons}

\subsection{Acyclic and Aromatic Hydrocarbons}

Estimation of $\Delta_{0}^{T_{\text {fus }}} S_{\text {tpce }}$ for acyclic and aromatic hydrocarbons (aah) can be achieved by summing the group values consistent with the structure of the molecule as illustrated by the following equation:

$$
\begin{gathered}
\Delta_{0}^{T_{\text {fus }}} S_{\text {tpce }}(\text { a } a h)=\sum_{i} n_{i} G_{i}+n_{\mathrm{CH}_{2}} C_{\mathrm{CH}_{2}} G_{\mathrm{CH}_{2}} ; \\
C_{\mathrm{CH}_{2}}=1.31 \text { when } n_{\mathrm{CH}_{2}} \geqslant \sum n_{i} ; \\
i \neq \mathrm{CH}_{2} \text { otherwise } C_{\mathrm{CH}_{2}}=1 .
\end{gathered}
$$

The group coefficient for a methylene group $C_{\mathrm{CH}_{2}}$ is used whenever the total number of consecutive methylene groups in a molecule $n_{\mathrm{CH}_{2}}$ equals or exceeds the sum of the other remaining groups $\Sigma n_{i}$. This applies to both hydrocarbons and all derivatives. In oligomers, and polymers, the decision as to whether to include this group coefficient should be based on the structure of the repeating unit. Some examples illustrating the use of both the groups in Table 1(a) and Eq. (2) are given in Table 3 and additional discussion regarding the use of $C_{\mathrm{CH}_{2}}$ is provided in the discussion that pertains to polymers below. Entries for each estimation in Table 3 include the melting point $T_{\text {fus }}$ and all transition temperatures $T_{t}$ for which there is a substantial enthalpy change. The estimated and experimental (in parentheses) phase change entropies follow. Similarly, the total phase change enthalpy calculated as the product of $\Delta_{0}^{T_{\text {fus }}} S_{\text {tpce }}$ and $T_{\text {fus }}$ is followed by the experimental total phase change enthalpy (or fusion enthalpy). Finally, details in estimating $\Delta_{0}^{T_{\text {fus }}} S_{\text {tpce }}$ for each compound are included as the last entry for each compound.

\subsubsection{Styrene}

The estimation of the fusion entropy of styrene is an example of an estimation of a typical aromatic hydrocarbon. Identification of the appropriate groups in Table 1(a) results in an entropy of fusion of $52.5 \mathrm{~J} \cdot \mathrm{mol}^{-1} \cdot \mathrm{K}^{-1}$ and together with the experimental melting point, an enthalpy of fusion of $12.6 \mathrm{~kJ} \cdot \mathrm{mol}^{-1}$ is estimated. This can be compared to the experimental value of $11.0 \mathrm{~kJ} \cdot \mathrm{mol}^{-1}$. It should be pointed out that the group values for aromatic molecules are purely additive while the group values for other cyclic $s p^{2}$ atoms, summarized in Table 1(b), are treated as corrections to the ring equation. This will be discussed in more detail below.

\subsubsection{1-Heptene}

The fusion entropy of 1-heptene is obtained in a similar fashion. In this case, the number of consecutive methylene groups in the molecule exceeds the sum of the remaining terms in the estimation and this necessitates the use of the group coefficient $C_{\mathrm{CH}_{2}}$ of 1.31 . For a molecule such as 3-heptene (estimation not shown), the group coefficient of 1.31 would not be applied. For a molecule such as 3-decene (also not shown), the group coefficient of 1.31 would be applied only to the five consecutive methylene groups. The remaining methylene group at carbon 2 would be treated normally $\left(C_{\mathrm{CH}_{2}}=1.0\right)$ and would not be counted in $\Sigma n_{i}$.

\subsubsection{Perylene}

Estimation of the phase change entropy of perylene provides an example of a molecule containing both peripheral and internal quaternary $s p^{2}$ carbon atoms adjacent to an $s p^{2}$ atom. The carbon atoms in graphite are another example of internal quaternary $s p^{2}$ carbon atoms. In the application of these group values to obtain the phase change properties of other aromatic molecules, it is important to remember that the aromatic portion of a molecule is defined in these estimations as molecules containing only benzenoid carbons and the corresponding nitrogen heterocycles. While a molecule like 1,2-benzacenaphthene (fluoranthene) would be considered aromatic, the five membered ring in acenaphtylene, according to this definition is not. Estimation of $\Delta_{0}^{T_{\text {fus }}} S_{\text {tpce }}$ for acenaphthylene will be illustrated below.

\subsection{Nonaromatic Cyclic and Polycyclic Hydrocarbons}

The protocol established for estimating $\Delta_{0}^{T_{\text {fus }}} S_{\text {tpce }}$ of unsubstituted cyclic hydrocarbons uses Eq. (3) to evaluate this term for the parent cycloalkane, $\Delta_{0}^{T_{\text {fus }}} S_{\text {tpce }}($ ring $)$. For substituted and polycyclic cycloalkanes,

$$
\Delta_{0}^{T_{\text {fus }}} S_{\text {tpce }}(\text { ring })=[33.4]+[3.7][n-3] ;
$$

$$
n=\text { number of ring atoms, }
$$

$$
\Delta_{0}^{T_{\text {fus }}} S_{\text {tpce }}(\text { ring })=[33.4] N+[3.7][R-3 N] ;
$$

$R=$ total number of ring atoms; $N=$ number of rings,

the results of Eqs. (3) or (4), respectively, are then corrected for the presence of substitution and hybridization patterns in the ring that differ from the standard cyclic secondary $s p^{3}$ pattern found in the parent monocyclic alkanes, $\Delta_{0}^{T_{\text {fus }}} S_{\text {tpce }}$ (corr $)$. These correction terms can be found in Table 1(b). Once these corrections are included in the estimation, any additional acyclic groups present as substitutents on the ring are added to the results of Eqs. (3) or (4) and $\Delta_{0}^{T_{\text {fus }}} S_{\text {tpce }}($ corr $)$. These additional acyclic and/or aromatic terms $\left[\Delta_{0}^{T_{\text {fus }}} S_{\text {tpce }}(a a h)\right]$ are added according to the protocol discussed above in the use of Eq. (2). The following ex- 
amples of Table 3 illustrate the use of Eq. (3) and (4) according to Eq. (5) to estimate the total phase change entropy of cyclic molecules $\Delta_{0}^{T_{\text {fus }}} S_{\text {tpce }}$ (total):

$$
\begin{aligned}
\Delta_{0}^{T_{\text {fus }}} S_{\text {tpce }}(\text { total })= & \Delta_{0}^{T_{\text {fus }}} S_{\text {tpce }}(\text { ring })+\Delta_{0}^{T_{\text {fus }}} S_{\text {tpce }}(\text { corr }) \\
& +\Delta_{0}^{T_{\text {fus }}} S_{\text {tpce }}(\text { a } a h) .
\end{aligned}
$$

\subsubsection{0,10,13,13-Tetramethyl-1,5-cyclohexadecadiyne}

The estimation of $\Delta_{0}^{T_{\text {fus }}} S_{\text {tpce }}$ for 10,10,13,13-tetramethyl1,5-cyclohexadecadiyne illustrates the use of Eq. (5) for a monocyclic alkyne. Once the hexadecane ring is estimated $([33.4]+13[3.7])$, correcting for the presence of two cyclic quaternary $s p^{3}$ carbon atoms $(2[-34.6])$, four cyclic $s p$ carbon atoms $(4[-4.7])$ and four methyl groups $(4[17.6])$ completes this estimation.

\subsubsection{Bullvalene}

Bullvalene, a tricyclic hydrocarbon, provides an example of the use of Eqs. (4) and (5). The minimum number of bonds that need to be broken to form a completely acyclic molecule is used to determine the number of rings. In this case it is three. Application of Eq. (4) to bullvalene $[3[33.4]+3.7[10-9]]$ provides $\Delta_{0}^{T_{\text {fus }}} S_{\text {tpce }}$ (ring). Addition of the contributions of the four cyclic tertiary $s p^{3}$ carbons and the six tertiary $s p^{2}$ carbons to the results of Eq. (4), $\Delta_{0}^{T_{\text {fus }}} S_{\text {tpce }}$ (corr), completes the estimation.

\subsubsection{Acenaphthylene}

Estimation of $\Delta_{0}^{T}$ fus $S_{\text {tpce }}$ and $\Delta_{0}^{T_{\text {fus }}} H_{\text {tpce }}$ for acenaphthylene completes this section on cyclic hydrocarbons. Molecules that contain rings fused to aromatic rings but are not completely aromatic, according to the definition provided above, are estimated by first calculating $\Delta_{0}^{T_{\text {fus }}} S_{\text {tpce }}$ (ring) for the contributions of the nonaromatic ring according to Eqs. (3) or (4). The atoms of the nonaromatic ring should be selected on the basis of the smallest number of ring atoms that account for all the nonaromatic carbons. This is then followed by addition of the adjustments for the nonsecondary $s p^{3}$ ring carbons, the contributions of the remaining aromatic groups and any other substitutents that may be present. In acenaphthylene, the contribution of the five membered ring $\left\{\Delta_{0}^{T_{\text {fus }}} S_{\text {tpce }}(\right.$ ring $\left.):[33.4]+2[3.7]\right\}$ is first adjusted for each nonsecondary $s p^{3}$ carbon atom in the ring $\left\{\Delta_{0}^{T_{\text {fus }}} S_{\text {tpce }}(\right.$ corr $\left.):+2[-1.6]+3[-12.3]\right\}$, and then the remainder of the aromatic portion of the molecule is added $\left\{\Delta_{0}^{T_{\text {fus }}} S_{\text {tpce }}(a a h):[-7.5]+6[7.4]\right\}$. In a molecule such as [2,2]-meta-cyclophane (estimation not shown), the acyclic ring the chosen to contain the fewest ring atoms, ten carbons in this instance. The six aromatic ring atoms that make up a portion of the ten membered ring are considered as cyclic $s p^{2}$ carbon atoms (four quaternary $s p^{2}$ and two tertiary $s p^{2}$ carbons). Addition of the contributions of the six remaining aromatic tertiary carbon atoms not included in the aliphatic ring completes this estimation.

\section{Estimations of Hydrocarbon Derivatives}

Estimations involving derivatives of hydrocarbons are performed in a fashion similar to hydrocarbons. The estimation consists of three parts: the contribution of the hydrocarbon component, that of the carbon(s) bearing the functional group(s), $\Sigma_{i} n_{i} C_{i} G_{i}$, and the contribution of the functional group(s) $\Sigma_{k} n_{k} C_{j} G_{k}$. The symbols $n_{i}, n_{k}$ refer to the number of groups of type $i$ and $k$. Acyclic and cyclic compounds are treated separately as before. For acyclic and aromatic molecules, the hydrocarbon portion is estimated using Eq. (2); cyclic or polycyclic molecules are estimated using Eqs. (3) and (4), respectively. Similarly, the contribution of the carbon(s) bearing the functional group(s) is evaluated from Tables 1 (a) or 1(b) modified by the appropriate group coefficient $C_{i}$ as will be illustrated below. The group values of the functional groups $G_{k}$ are listed in Tables 2(a) and 2(b). The corresponding group coefficient $C_{j}$ is equal to one for all functional groups except those identified otherwise in Table 2(a). Selection of the appropriate value of $C_{j}$ from Table 2(a) is based on the total number of functional groups and is discussed below. Functional groups that make up a portion of a ring are listed in Table 2(b). The use of these values in estimations will be illustrated separately. Equations (6) and (7) summarize the protocol developed to estimate $\Delta_{0}^{T_{\text {fus }}} S_{\text {tpce }}($ total) for acyclic and aromatic derivatives and for cyclic and polycyclic hydrocarbon derivatives, respectively,

$$
\begin{aligned}
\Delta_{0}^{T_{\text {fus }}} S_{\text {tpce }}(\text { total })= & \Delta_{0}^{T_{\text {fus }}} S_{\text {tpce }}(\text { aah })+\sum_{i} n_{i} C_{i} G_{i} \\
& +\sum_{k} n_{k} C_{j} G_{k}, \\
\Delta_{0}^{T_{\text {fus }}} S_{\text {tpce }}(\text { total })= & \Delta_{0}^{T_{\text {fus }}} S_{\text {tpce }}(\text { ring })+\Delta_{0}^{T_{\text {fus }}} S_{\text {tpce }}(\text { corr }) \\
& +\sum_{i} n_{i} C_{i} G_{i}+\sum_{k} n_{k} C_{j} G_{k}
\end{aligned}
$$

where

$$
C_{j}=\sum_{k} n_{k} .
$$

In view of the large number of group values listed in Tables 2(a) and 2(b), selection of the appropriate functional group(s) is particularly important. Four functional groups in Table 2(a), chlorine, the hydroxyl and carboxyl group, and trisubstituted amides are dependent on the total substitution pattern in the molecule. Coefficients for these four groups $C_{j}$ are available for molecules containing up to six functional groups. Selection of the appropriate value of $C_{j}$ for one of these four functional groups is based on the total number of functional groups in the molecule. Estimations of the fusion entropy of polymers suggests that the group coefficient for $C_{6}$ in Table 2(b), is adequate for molecules containing more than a total of six functional groups. ${ }^{19}$ 


\subsection{Acyclic and Aromatic Hydrocarbon Derivatives}

The estimations for decachlorobiphenyl, N-acetyl-Lalanine amide, 2,2,2-trifluoroacetonitrile, and isoquinoline, shown in Table 4(a), illustrate the estimations of substituted aromatic and acyclic hydrocarbon derivatives.

\subsubsection{Decachlorobiphenyl}

Decachlorobiphenyl is an example of an estimation of a polysubstituted aromatic molecule. Selection of the value for a quaternary aromatic $s p^{2}$ carbon from Table 1(a) depends on the nature of the functional group attached to carbon. If the functional group at the point of attachment is $s p^{2}$ hybridized or contains nonbonding electrons, the value for a "peripheral aromatic $s p^{2}$ carbon adjacent to an $s p^{2}$ atom" is selected. Otherwise a "peripheral aromatic $s p^{2}$ carbon adjacent to an $s p^{3}$ atom" is used. The remainder of the estimation follows the guidelines outlined above with the exception that chlorine is one of the four functional groups whose group coefficient $C_{j}$ depends on the degree of substitution ( $C_{6}$ is used in this example).

\subsection{2. $\mathrm{N}$-acetyl-L-alanine amide}

The estimation of $\Delta_{0}^{T_{\text {fus }}} S_{\text {tpce }}$ for N-acetyl-L-alanine amide follows directly from Eq. (6). The molecule contains both a primary and secondary amide linkage. The asymmetric center is a tertiary carbon that contains two functional groups attached to it and as such its contribution is attenuated by the group coefficient for a tertiary carbon. Addition of the contributions of the two methyl groups completes the estimation.

\subsubsection{Trifluoroacetonitrile}

The estimation of $\Delta_{0}^{T}$ fus $S_{\text {tpce }}$ for trifluoroacetonitrile illustrates an example of a molecule containing fluorine. The group value for a fluorine on a trifluoromethyl group in Table 2(a) is given per fluorine atom. The contribution of the quaternary carbon atom when attached to functional groups is attenuated by the group coefficient $C_{i}$. Inclusion of the group value for a thiol completes this estimation. When fluorine is combined with the functional groups listed in Table 2(b), the group coefficient chosen should be based on the presence of fluorine as a single functional group, regardless of the number of fluorine atoms present. For example, a molecule such as trifluoromethanol would be considered to contain two functional groups.

\subsubsection{Isoquinoline}

The estimation of isoquinoline illustrates an example of another aromatic molecule. The only exception in this case is the need to substitute the group value for a heterocyclic aromatic amine. Otherwise the same protocol is followed as in the estimation of naphthalene (not shown).

\subsection{Cyclic and Polycyclic Hydrocarbon Derivatives}

The protocol for estimating the total phase change properties of cyclic and polycyclic molecules also follows from the procedure described above for the corresponding cyclic hydrocarbons. In cyclic molecules, the substituent or functional group may be attached to the ring or it may be part of the ring. If the functional group is part of the ring, the group values listed in Table 2(b) are to be used. The procedure first involves estimating $\Delta_{0}^{T_{\text {fus }}} S_{\text {tpce }}$ for the corresponding hydrocarbon ring, then correcting for the heterocyclic component(s), and if necessary, correcting the ring carbons attached to the cyclic functional group by the appropriate group coefficients. This is illustrated in Table 4(b) by the following examples.

\subsubsection{2-Chlorodibenzodioxin}

The dioxane ring of 2-chlorodibenzodioxin is treated as being a derivative of cyclohexane. According to Eq. (7), the ring equation is first used to estimate the contributions of the cyclohexane ring. This ring contains two cyclic ether oxygens and four quaternary cyclic $s p^{2}$ carbon atoms and must be modified accordingly. The remaining eight carbon atoms are treated as aromatic carbons and values appropriate to their substitution pattern are chosen. The addition of the contribution of the chlorine completes the estimation.

\subsubsection{6,8,9-Trimethyladenine}

6,8,9-Trimethyladenine is estimated in a similar fashion. The ring equation [Eq. (3)] is used first to generate the contribution of the five membered heterocyclic ring. In this instance the ring has been modified by the addition of a cyclic $s p^{2}$ hybridized nitrogen atom and a nitrogen which comprises part of a cyclic tertiary amine. Both ring substitutions require appropriate corrections. The hybridization and substitution of the remaining three cyclic carbon atoms of the five membered ring have likewise been changed from the pattern found in cyclopentane and appropriate changes must also be included in $\Delta_{0}^{T_{\text {fus }}} S_{\text {tpce }}$ (corr). The remaining four ring atoms comprise a portion of an aromatic ring; their contributions can be added directly. The two nitrogen atoms make up a portion of the heterocyclic aromatic ring along with a quaternary and tertiary aromatic $s p^{2}$ carbon atom. The quaternary aromatic $s p^{2}$ carbon atom is attached to an exocyclic nitrogen atom with a lone pair of electrons and consequently, the quaternary aromatic carbon is treated as being adjacent to an $s p^{2}$ center. The contributions of the tertiary aromatic $s p^{2}$ carbon atom, the methyl groups, and the acyclic secondary amine complete the estimation.

\subsubsection{Lenacil}

Estimations of Lenacil (3-cyclohexyl-6,7-dihydro-1Hcyclopentapyrimidine-2,4-(3H,5H)-dione) require some thought in properly identifying the functional groups in the molecule. The functional group that makes up a portion of the pyrimidine-2,4-dione ring in this molecule cannot be 
found directly in Table 2(b). It must therefore be simplified and this simplification can be accommodated in various ways. The ring can be considered to be a combination of either an adjacent cyclic imide (-CONRCO-) and cyclic amide nitrogen $(-\mathrm{NH}-)$, a cyclic urea $(-\mathrm{NRCONH}-)$ and amide carbonyl (-CO-), or a cyclic secondary and tertiary amide. An examination of the available groups in Table 2(b) will reveal that although group values for cyclic imides are available (-NRCONH-, $-\mathrm{NRCONR}-)$, there is no appropriate group available for an $\mathrm{N}$-substituted cyclic nitrogen of an amide. Similarly, group values for a cyclic urea and amide carbonyl are not available. The most appropriate group values that are available are for cyclic amides. Once the appropriate group is identified, the procedure follows the same protocol as established for other polycyclic molecules.

\subsubsection{Cortisone}

The estimation of the fusion enthalpy of cortisone illustrates an example of an estimation of a complex polycyclic compound. This tetracyclic 17 atom ring system contains three cyclic quaternary centers $(3[-34.6])$, three cyclic tertiary $s p^{3}$ centers, $(3[-14.7])$, a cyclic tertiary $s p^{2}$ center which is attached to a functional group [1.92][-1.6], a quaternary $s p^{2}$ center $([-12.3])$ as well as two cyclic carbonyl group (2[-1.4]). Addition of these modifications to the ring equation $(4[33.4]+5[3.7])$ estimates the contributions of the ring. Addition of the contributions of the substituents which include three hydroxyls ((3)(12.1)[1.7]), two methyls (2[17.6]), a methylene ([7.1]), and a carbonyl group of an acyclic ketone ([4.6]) completes the estimation. The molecule contains five functional groups, hence $\mathrm{C}_{5}$ for a hydroxyl group is used.

\subsection{Polymers}

In addition to the estimation of $\Delta_{0}^{T_{\text {fus }}} S_{\text {tpce }}$ of small molecules, the parameters of Tables 1 and 2 can be used to predict $\Delta_{0}^{T_{\text {fus }}} S_{\text {tpce }}$ and $\Delta_{0}^{T_{\text {fus }}} H_{\text {tpce }}$ (when the experimental melting point is known) of crystalline oligomers and linear polymers. Since the parameters in Tables 1 and 2 differ slightly from those reported previously, ${ }^{19}$ the predictions of Eqs. (2)(6) likewise produce slightly different results. However a similar overall correlation (slightly improved) between experimental and calculated results is obtained using the updated parameters. The protocol used to evaluate $\Delta_{0}^{T_{\text {fus }}} S_{\text {tpce }}$ of polymers is exactly the same as outlined above. In this instance, the entropic value is calculated on the basis of the structure of the repeat unit of the polymer. Best correlations are obtained when the group coefficient $C_{k}$ chosen is based on the number of functional groups present on the repeat unit and on the two nearest neighbors. The polymer $\left(\mathrm{CH}_{2} \mathrm{O}\right)_{n}$, is treated as an infinite chain with $n_{0}=n_{\mathrm{CH}_{2}}$. For a molecule such as $\mathrm{CH}_{3} \mathrm{O}\left(\mathrm{CH}_{2} \mathrm{CH}_{2} \mathrm{O}\right)_{n} \mathrm{CH}_{3}$, the number of methylene groups in the repeat unit exceeds the number of oxygens and therefor the group coefficient for a methylene group should be used. As $n$ becomes smaller, a point will be reached when the molecule no longer represents an oligomer. In this in- stance the group coefficient for a methylene group should be dropped. This should occur when $n$ becomes less than the number of other groups that make up the remainder of the molecule. In the case just described, this would occur when $n$ becomes less than three.

The column entries in Tables 6 and 7 are identical (these data were not used in generating the group values of Tables 1 and 2) and are described below. Calculated and experimental values of $\Delta_{0}^{T_{\text {fus }}} S_{\text {tpce }}$ for a series of linear polymers are provided in Table 6.

\section{The Group Coefficient in Cycloalkyl Derivatives}

The protocol in determining whether to use the group coefficient $C_{\mathrm{CH}_{2}}$ depends on whether the number of consecutive methylene groups exceeds the sum of the remaining groups excluding other methylene groups in the count. In an estimation of a cyclic derivative, the contribution of the ring is determined by Eq. (3) or (4) along with other terms necessary to correct for substitution and hybridization changes. This will vary depending on the nature of the ring and its substitution patterns. Fewer terms are necessary to estimate the total phase change entropy of ethylcyclohexane than ethylcyclohexadiene, even though in principle, both contain the same number of groups. To avoid any ambiquity in determining when to use this group coefficient, the number of groups associated with a ring structure should be determined by the size of the ring and the number of substituents or functional groups attached to the ring. For example, a molecule such as 2,5-di- $n$-undecyloxy-1,4-benzoquinone, contains 10 adjacent methylene groups. These methylene groups should be compared to the total number of other groups on the molecule. This includes two carbonyls, two methyl groups, two ether oxygens, and four $s p^{2}$ hybridized carbon atoms, adding up to a total of 10. Since these two numbers are equal, the group coefficient should be applied to both undecyl groups.

\section{Polymorphism}

In some cases, particularly with some pharmaceuticals, different fusion enthalpies and melting points have been reported for the same material. For example, fusion enthalpies of $18284\left(428.2 \mathrm{~K}^{20}\right.$ and $23810 \mathrm{~J} \mathrm{~mol}^{-1}(430.3 \mathrm{~K})^{21}$ have been reported for codeine. While one of these values may be in error, the two values may represent accurate physical properties of different crystalline modifications of codeine. The value estimated by the group additivity approach described above generally gives total phase change entropies and enthalpies associated with the most stable modification at the melting point. A recent review article summarizes pharmaceuticals known to exhibit polymorphism. ${ }^{22}$ 


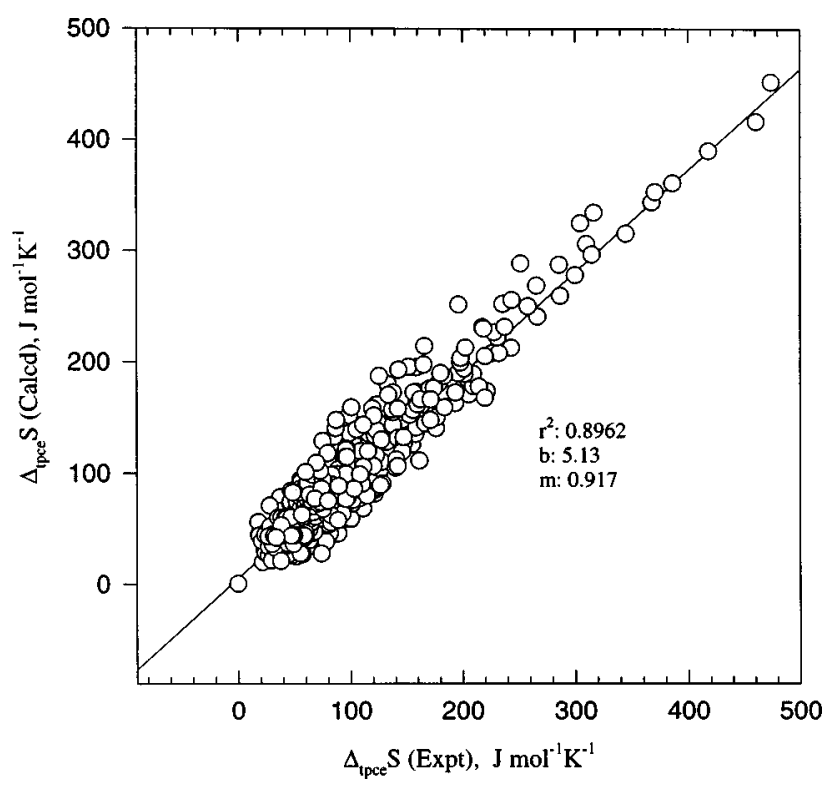

FIG. 3. A comparison of calculated and experimental $\Delta_{0}^{T_{\text {fus }}} S_{\text {tpce }}$ of 1858 database compounds.

\section{Statistics of the Correlation}

\subsection{Database Compounds}

The group values included in Tables 1 and 2 were generated from the fusion entropies of a total of 1858 compounds. Melting and transition temperatures (column 1), experimental enthalpies associated with all solid-solid and solid-liquid phase changes ( $\Delta H_{\mathrm{pce}}$, column 2), the corresponding phase change entropies $\left(\Delta S_{\text {pce }}\right.$, column 3$)$, the total experimental phase change entropy (column 4) and enthalpy (columns 6), and the corresponding values estimated from the group values of Tables 1 and 4 (columns 5 and 7) for each of these compounds are given in Table 5. A summary of each calculation is also included in the form of the alphanumeric terms

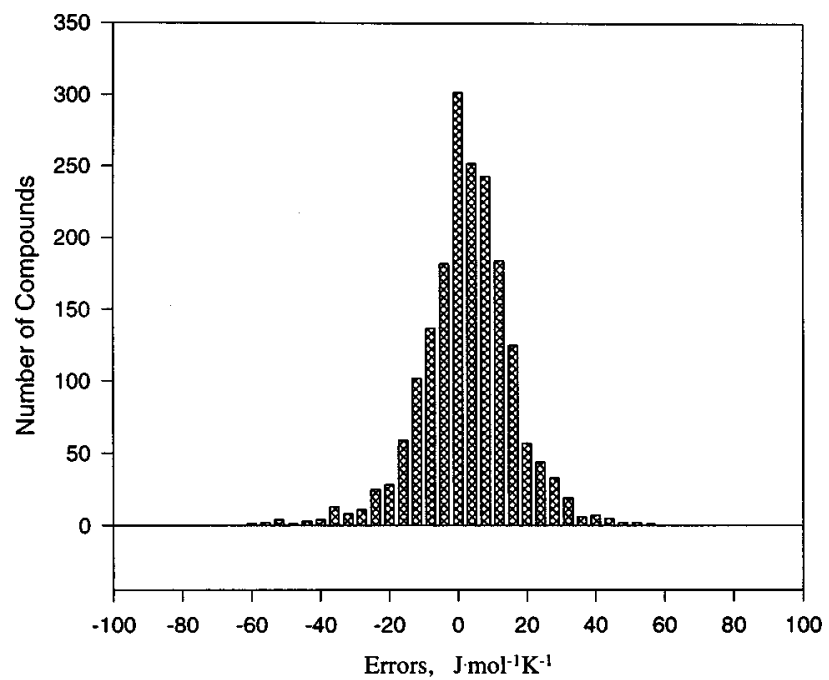

FIG. 4. A histogram illustrating the distribution of errors in estimating $\Delta_{0}^{T_{\text {fus }}} S_{\text {tpce }}$ of the database compounds.

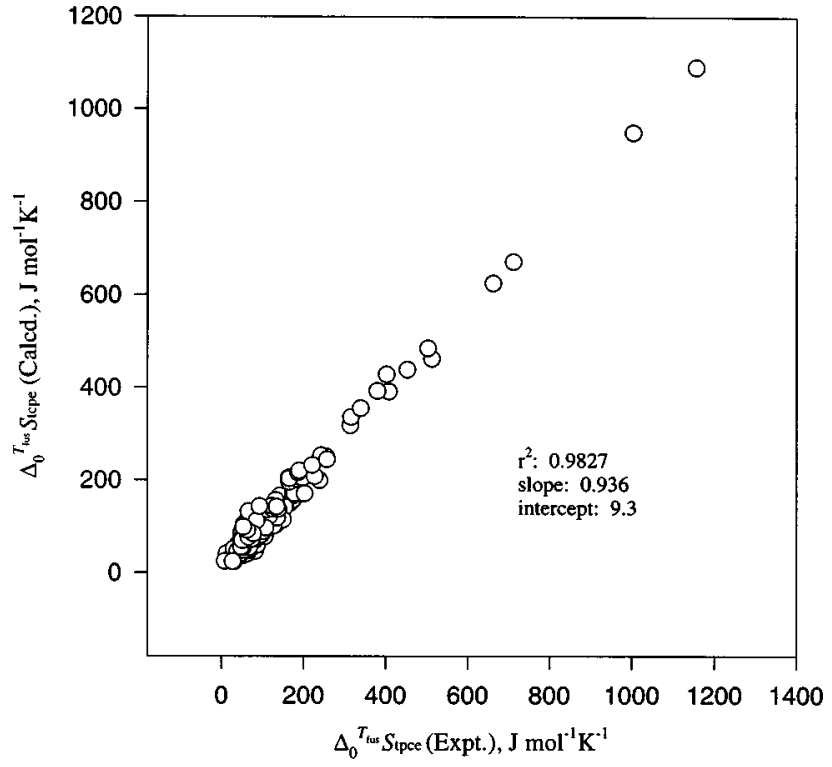

FIG. 5. A comparison of calculated and experimental $\Delta_{0}^{T_{\text {fus }}} S_{\text {tpce }}$ of 260 test compounds.

used in each calculation. These alphanumeric terms are defined in Tables 1 and 2 for each group (in parenthesis). Table 5 also includes a number of compounds that were not included in deriving either the statistics or the group values. Reasons for this are noted in the table. An asterisk following the molecular formula in the table identifies these materials. Experimental and calculated total phase change entropies for the database are compared in Fig. 3. The correlation was characterized by the slope $m$, intercept $b$, and correlation coefficient $\left(r^{2}\right)$ given in the figure. A histogram of the errors associated with this correlation is shown in Fig. 4. The absolute average and relative errors between experimental and

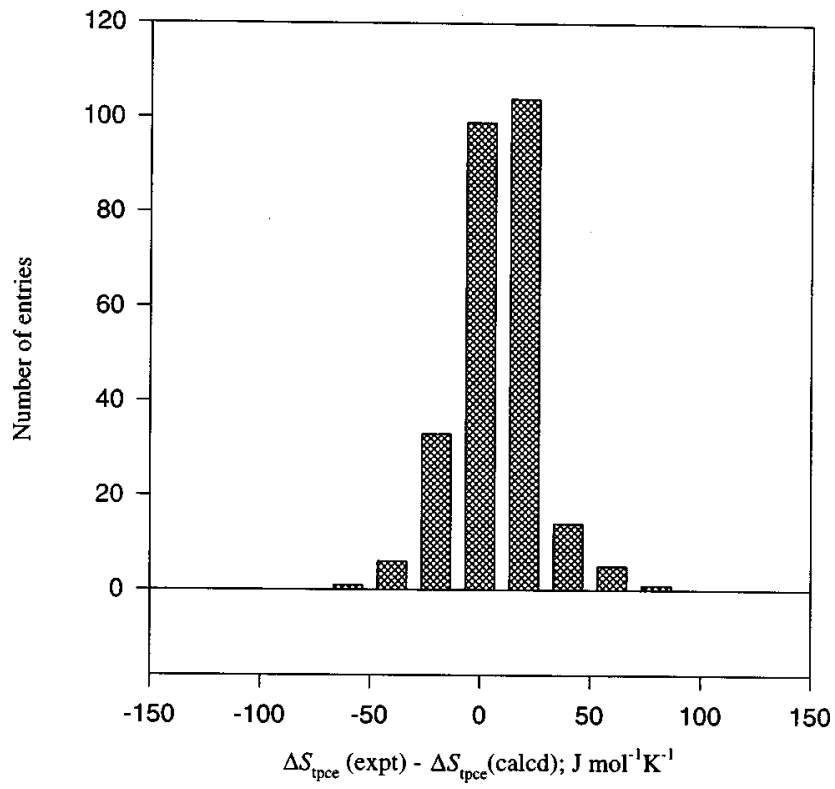

FIG. 6. A histogram illustrating the distribution of errors in estimating $\Delta_{0}^{T_{\text {fus }}} S_{\text {tpce }}$ of 260 test compounds. 
calculated $\Delta_{0}^{T_{\text {fus }}} S_{\text {tpce }}$ and $\Delta_{0}^{T_{\text {fus }}} H_{\text {tpce }}$ values for these 1858 compounds are $9.9 \mathrm{~J} \cdot \mathrm{mol}^{-1} \cdot \mathrm{K}^{-1}$ and $3.52 \mathrm{~kJ} \cdot \mathrm{mol}^{-1}$, and 0.154 and 0.17 , respectively. The standard deviations between experimental and calculated values for $\Delta_{0}^{T_{\text {fus }}} S_{\text {tpce }}$ and $\Delta_{0}^{T_{\text {fus }}} H_{\text {tpce }}$ are $\pm 13.0 \mathrm{~J} \cdot \mathrm{mol}^{-1} \cdot \mathrm{K}^{-1}$ and $\pm 4.88 \mathrm{~kJ} \cdot \mathrm{mol}^{-1}$, respectively. An additional 60 compounds exhibited errors exceeding 3 s.d. and were excluded from the correlations and from Figs. 3 and 4. These compounds are included in Tables 5 and 7.

\subsection{Test Compounds}

In addition to the 1858 compounds that make up the database, an additional 260 compounds have been used as test materials to provide an unbiased evaluation of the reliability of the group values given in Tables 1 and 2 . These fusion enthalpies include compounds obtained from more recent searches of the literature and are reported in Table 7. The data included in Table 7 are in the same format as the data in Table 5. The correlation between experimental and calculated values for the test compounds is shown in Fig. 5. The standard deviations between experimental and calculated values for $\Delta_{0}^{T_{\text {fus }}} S_{\text {tpce }}$ and $\Delta_{0}^{T_{\text {fus }}} H_{\text {tpce }}$ were $\pm 18.4 \mathrm{~J} \cdot \mathrm{mol}^{-1}$ $\cdot \mathrm{K}^{-1}$ and $\pm 7.2 \mathrm{~kJ} \cdot \mathrm{mol}^{-1}$, respectively. The absolute average and relative errors between experimental and calculated $\Delta_{0}^{T_{\text {fus }}} S_{\text {tpce }}$ and $\Delta_{0}^{T_{\text {fus }}} H_{\text {tpce }}$ values for these 260 compounds were $13.9 \mathrm{~J} \cdot \mathrm{mol}^{-1} \cdot \mathrm{K}^{-1}$ and $5.28 \mathrm{~kJ} \cdot \mathrm{mol}^{-1}$, and 0.181 and 0.194 , respectively. In addition to these 260 compounds, some recently acquired data are also included in Table 7 . As before, compounds not included in the correlations are identified by an asterisk following their molecular formula (see Tables 5, 6, and 7). References to Tables 5, 6, and 7 are listed in Table 8.

TABLE 1. (a) Contributions of the hydrocarbon portion of acyclic and aromatic molecules

\begin{tabular}{|c|c|c|c|c|c|}
\hline \multirow{2}{*}{$\begin{array}{l}\text { Acyclic and aromatic carbon groups } \\
\text { primary } s p^{3}\end{array}$} & \multicolumn{3}{|c|}{$\begin{array}{c}\text { Group value } \mathrm{a}^{\mathrm{a}} \\
G_{i}\left(\mathrm{~J} \cdot \mathrm{mol}^{-1} \cdot \mathrm{K}^{-1}\right)\end{array}$} & \multicolumn{2}{|c|}{$\begin{array}{c}\text { Group coefficients }^{\mathrm{a}} \\
C_{i}\end{array}$} \\
\hline & $\mathrm{CH}_{3}-$ & 17.6 & $(A 1)$ & & \\
\hline secondary $s p^{3}$ & $>\mathrm{CH}_{2}$ & 7.1 & $(A 2)$ & $1.31^{\mathrm{b}}$ & $(B 2)$ \\
\hline tertiary $s p^{3}$ & $-\mathrm{CH}<$ & -16.4 & $(A 3)$ & 0.60 & (B3) \\
\hline quaternary $s p^{3}$ & $>\mathrm{C}<$ & -34.8 & $(A 4)$ & 0.66 & $(B 4)$ \\
\hline secondary $s p^{2}$ & $=\mathrm{CH}_{2}$ & 17.3 & $(A 5)$ & & \\
\hline tertiary $s p^{2}$ & $=\mathrm{CH}-$ & 5.3 & $(A 6)$ & 0.75 & $(B 6)$ \\
\hline quaternary $s p^{2}$ & $=\mathrm{C}(\mathrm{R})-$ & -10.7 & $(A 7)$ & & \\
\hline tertiary $s p$ & $\mathrm{H}-\mathrm{C} \equiv$ & 14.9 & $(A 8)$ & & \\
\hline quaternary $s p$ & $-\mathrm{C} \equiv$ & -2.8 & (A9) & & \\
\hline aromatic tertiary $s p^{2}$ & $=\mathrm{C}_{a} \mathrm{H}-$ & 7.4 & $(A 10)$ & & \\
\hline $\begin{array}{l}\text { quaternary aromatic } s p^{2} \text { carbon } \\
\text { adjacent to an } s p^{3} \text { atom }\end{array}$ & $=\mathrm{C}_{a}(\mathrm{R})-$ & -9.6 & $(A 11)$ & & \\
\hline $\begin{array}{l}\text { peripheral quaternary aromatic } s p^{2} \\
\text { carbon adjacent to an } s p^{2} \text { atom }\end{array}$ & $=\mathrm{C}_{a}(\mathrm{R})-$ & -7.5 & $(A 12)$ & & \\
\hline $\begin{array}{l}\text { internal quaternary aromatic } s p^{2} \\
\text { carbon adjacent to an } s p^{2} \text { atom }\end{array}$ & $=\mathrm{C}_{a}(\mathrm{R})-$ & -0.7 & (A13) & & \\
\hline
\end{tabular}

${ }^{a}$ The alphanumeric terms, $A 1, A 2, B 2, \ldots$ are a device used to identify each group value in the estimations provided in Tables 7,8 , and 9 .

${ }^{b}$ The group coefficient of 1.31 for $\mathrm{C}_{\mathrm{CH}_{2}}$ is applied only when the number of consecutive methylene groups equals or exceeds the sum of the remaining groups; see Eq. 2 in text.

TABLE 1. (b) Contributions of the cyclic hydrocarbon portions of the molecule

\begin{tabular}{|c|c|c|c|c|c|}
\hline Contributions of cyclic carbons & \multicolumn{3}{|c|}{$\begin{array}{l}\text { Group value }\left(G_{i}\right) \\
\left(\mathrm{J} \cdot \mathrm{mol}^{-1} \cdot \mathrm{K}^{-1}\right)\end{array}$} & \multicolumn{2}{|c|}{$\begin{array}{l}\text { Group coefficient } \\
\qquad C_{i}\end{array}$} \\
\hline \multicolumn{6}{|c|}{ Ring equations for nonaromatic cyclic compounds } \\
\hline \multicolumn{6}{|c|}{$\Delta S_{\text {ring }}=[33.4(A 14)]+[3.7(A 15)][n-3] ; \quad n=$ number of ring atoms } \\
\hline \multicolumn{6}{|c|}{ Ring equation for nonaromatic polycyclic compounds } \\
\hline \multicolumn{4}{|c|}{ 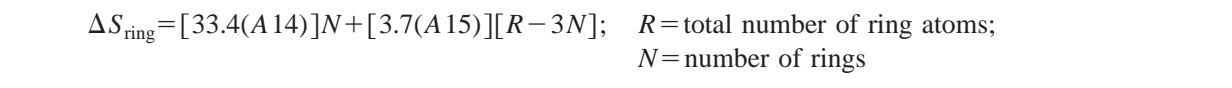 } & & \\
\hline cyclic tertiary $s p^{3}$ & $>\mathrm{C}_{\mathrm{c}} \mathrm{H}(R)$ & -14.7 & $(A 16)$ & & \\
\hline cyclic quaternary $s p^{3}$ & $>\mathrm{C}_{c}(R)_{2}$ & -34.6 & $(A 17)$ & & \\
\hline cyclic tertiary $s p^{2}$ & $=\mathrm{C}_{c} \mathrm{H}_{-}$ & -1.6 & $(A 18)$ & 1.92 & $(B 18)$ \\
\hline cyclic quaternary $s p^{2}$ & $=\mathrm{C}_{c}(R)-$ & -12.3 & $(A 19)$ & & \\
\hline cyclic quaternary $s p$ & $=\mathrm{C}_{c}=; R-\mathrm{C}_{c} \equiv$ & -4.7 & $(A 20)$ & & \\
\hline
\end{tabular}


TABLE 2. (a) Contributions of the functional group portion of the molecule

\begin{tabular}{|c|c|c|c|c|c|c|c|c|}
\hline \multirow{2}{*}{ Functional groups ${ }^{\mathrm{a}}$} & \multirow{2}{*}{\multicolumn{3}{|c|}{$\begin{array}{l}\text { Group value }\left(G_{k}\right)^{\mathrm{a}} \\
\mathrm{J} /(\mathrm{mol} \mathrm{K})\end{array}$}} & \multicolumn{5}{|c|}{$\begin{array}{c}\text { Group coefficient }\left(C_{k}\right)^{\mathrm{b}} \\
k\end{array}$} \\
\hline & & & & 2 & 3 & 4 & 5 & 6 \\
\hline bromine & $\mathrm{R}-\mathrm{Br}$ & 17.5 & $(A 21)$ & & & & & \\
\hline chlorine & $\mathrm{R}-\mathrm{Cl}$ & 10.8 & $(A 22)$ & $\begin{array}{c}1.5 \\
(B 22)\end{array}$ & $\begin{array}{c}1.5 \\
(C 22)\end{array}$ & $\begin{array}{c}1.5 \\
(D 22)\end{array}$ & $\begin{array}{c}1.5 \\
(E 22)\end{array}$ & $\begin{array}{c}1.5 \\
(F 22)\end{array}$ \\
\hline fluorine on an $s p^{2}$ carbon & $=\mathrm{CRF}$ & 19.5 & $(A 23)$ & & & & & \\
\hline fluorine on an aromatic carbon & $=\mathrm{CF}-$ & 16.6 & $(A 24)$ & & & & & \\
\hline 3-fluorines on an $s p^{3}$ carbon & $\mathrm{CF}_{3}-\mathrm{R}$ & 13.3 & $(A 25)$ & & & & & \\
\hline 2-flurorines on an $s p^{3}$ carbon & $\mathrm{R}-\mathrm{CF}_{2}-\mathrm{R}$ & 16.4 & $(A 26)$ & & & & & \\
\hline 1-fluorine on an $s p^{3}$ carbon & $\mathrm{R}-\mathrm{CF}-(\mathrm{R})_{2}$ & 12.7 & $(A 27)$ & & & & & \\
\hline fluorine on a ring carbon & $-\mathrm{CHF}-$ & {$[17.5]$} & $(A 28)$ & & & & & \\
\hline & $-\mathrm{CF}_{2}-$ & {$[17.5]$} & $(A 28)$ & & & & & \\
\hline iodine & $\mathrm{R}-\mathrm{I}$ & 19.4 & $(A 29)$ & & & & & \\
\hline hydroxyl group & $\mathrm{R}-\mathrm{OH}$ & 1.7 & $(A 30)$ & $\begin{array}{c}10.4 \\
(B 30)\end{array}$ & $\begin{array}{c}9.7 \\
(C 30)\end{array}$ & $\begin{array}{c}13.1 \\
(D 30)\end{array}$ & $\begin{array}{c}12.1 \\
(E 30)\end{array}$ & $\begin{array}{l}13.1 \\
(F 30)\end{array}$ \\
\hline phenol & $=\mathrm{C}-(\mathrm{OH})-$ & 20.3 & $(A 31)$ & & & & & \\
\hline ether & $\mathrm{R}-\mathrm{O}-\mathrm{R}$ & 4.71 & $(A 32)$ & & & & & \\
\hline peroxide, 1 & $\mathrm{R}-\mathrm{O}-\mathrm{O}-\mathrm{R}$ & {$[10.6]$} & $(A 33)$ & & & & & \\
\hline aldehyde & $\mathrm{R}-\mathrm{CH}(=\mathrm{O})$ & 21.5 & $(A 34)$ & & & & & \\
\hline ketone & $\mathrm{R}-\mathrm{C}(=\mathrm{O})-(\mathrm{R})$ & 4.6 & $(A 35)$ & & & & & \\
\hline carboxylic acid & $\mathrm{R}-\mathrm{C}(=\mathrm{O}) \mathrm{OH}$ & 13.4 & $(A 36)$ & $\begin{array}{c}1.21 \\
(B 36)\end{array}$ & $\begin{array}{l}2.25 \\
(C 36)\end{array}$ & $\begin{array}{l}2.25 \\
(D 36)\end{array}$ & $\begin{array}{l}2.25 \\
(E 36)\end{array}$ & $\begin{array}{l}2.25 \\
(F 36)\end{array}$ \\
\hline formate ester & $\mathrm{R}-\mathrm{OCH}(=\mathrm{O})$ & {$[4.2]$} & $(A 37)$ & & & & & \\
\hline ester & $\mathrm{R}-\mathrm{C}(=\mathrm{O}) \mathrm{O}-\mathrm{R}$ & 7.7 & $(A 38)$ & & & & & \\
\hline anhydride & $\mathrm{R}-\mathrm{C}(=\mathrm{O}) \mathrm{OC}(=\mathrm{O})-\mathrm{R}$ & {$[10.0]$} & $(A 39)$ & & & & & \\
\hline acyl chloride & $\mathrm{R}-\mathrm{C}(=\mathrm{O}) \mathrm{Cl}$ & {$[25.8]$} & $(A 40)$ & & & & & \\
\hline aromatic heterocyclic amine & $=\mathrm{N}-$ & {$[10.9]$} & $(A 41)$ & & & & & \\
\hline acyclic $s p^{2}$ nitrogen & $=\mathrm{N}-$ & {$[-1.8]$} & $(A 42)$ & & & & & \\
\hline tertiary amine & $\mathrm{R}-\mathrm{N}\left(\mathrm{R}_{2}\right)$ & -22.2 & $(A 43)$ & & & & & \\
\hline secondary amine & $\mathrm{R}-\mathrm{NH}-\mathrm{R}$ & -5.3 & $(A 44)$ & & & & & \\
\hline primary amine & $\mathrm{R}-\mathrm{NH}_{2}$ & 21.4 & $(A 45)$ & & & & & \\
\hline azide & $\mathrm{R}-\mathrm{N}_{3}$ & {$[-32.5]$} & $(A 46)$ & & & & & \\
\hline tertiary amine $N$-nitro & $\mathrm{R}_{2}-\mathrm{N}-\left(\mathrm{NO}_{2}\right)$ & 5.39 & $(A 47)$ & & & & & \\
\hline aliphatic secondary amine $N$-nitro & $\mathrm{R}-\mathrm{NH}-\left(\mathrm{NO}_{2}\right)$ & {$[-4.59]$} & $(A 48)$ & & & & & \\
\hline aromatic tertiary amine- $N$-nitro & $\mathrm{R}-\mathrm{NH}-\left(\mathrm{NO}_{2}\right)$ & {$[-41.7]$} & $(A 49)$ & & & & & \\
\hline nitro group & $\mathrm{R}-\mathrm{NO}_{2}$ & 17.7 & $(A 50)$ & & & & & \\
\hline$N$-nitro & $>\mathrm{N}-\left(\mathrm{NO}_{2}\right)$ & 39.8 & $(A 51)$ & & & & & \\
\hline N-nitroso & $>\mathrm{N}-\mathrm{N}=\mathrm{O}$ & {$[28.6]$} & $(A 52)$ & & & & & \\
\hline oxime & $=\mathrm{N}-\mathrm{OH}$ & {$[13.6]$} & $(A 53)$ & & & & & \\
\hline azoxy nitrogen & $\mathrm{N}=\mathrm{N}(\rightarrow \mathrm{O})-$ & {$[6.8]$} & $(A 54)$ & & & & & \\
\hline nitrate ester & $\mathrm{R}-\mathrm{ONO}_{2}$ & {$[24.4]$} & $(A 55)$ & & & & & \\
\hline nitrile & $\mathrm{R}-\mathrm{C} \equiv \mathrm{N}$ & 17.7 & $(A 56)$ & & & & & \\
\hline isocyanide & $\mathrm{R}-\mathrm{NC}$ & {$[17.5]$} & $(A 57)$ & & & & & \\
\hline isocyanate & $\mathrm{R}-\mathrm{N}=\mathrm{C}=\mathrm{O}$ & {$[23.1]$} & $(A 58)$ & & & & & \\
\hline tertiary amides & $\mathrm{R}-\mathrm{C}(=\mathrm{O}) \mathrm{NR}_{2}$ & -11.2 & $(A 59)$ & & & & & \\
\hline secondary amides & $\mathrm{R}-\mathrm{C}(=\mathrm{O}) \mathrm{NH}-\mathrm{R}$ & 1.5 & $(A 60)$ & & & & & \\
\hline primary amide & $\mathrm{R}-\mathrm{CONH}_{2}$ & 27.9 & $(A 61)$ & & & & & \\
\hline $\mathrm{N}, \mathrm{N}$-dialkylformamide, 1 & $\mathrm{HC}(=\mathrm{O}) \mathrm{NR}_{2}$ & {$[6.9]$} & $(A 62)$ & & & & & \\
\hline tetra substituted urea & $\mathrm{R}_{2} \mathrm{NC}(=\mathrm{O}) \mathrm{NR}_{2}$ & {$[-19.3]$} & $(A 63)$ & & & & & \\
\hline 1,1,3-trisubst urea & $\mathrm{R}_{2} \mathrm{NC}(=\mathrm{O}) \mathrm{NH}-\mathrm{R}$ & {$[0.2]$} & $(A 64)$ & $\begin{array}{l}-12.8 \\
(B 64)\end{array}$ & $\begin{array}{c}-24 \\
(C 64)\end{array}$ & $\begin{array}{c}6 \\
(D 64)\end{array}$ & & \\
\hline 1,1-disubstituted urea & $\mathrm{R}_{2} \mathrm{NC}(=\mathrm{O}) \mathrm{NH}_{2}$ & {$[19.5]$} & $(A 65)$ & & & & & \\
\hline 1,3-disubstituted urea & $\mathrm{RNHC}(=\mathrm{O}) \mathrm{NH}-\mathrm{R}$ & {$[1.5]$} & $(A 66)$ & & & & & \\
\hline mono substituted urea & $\mathrm{R}-\mathrm{NHC}(=\mathrm{O}) \mathrm{NH}_{2}$ & {$[22.5]$} & $(A 67)$ & & & & & \\
\hline N,N-disubstituted carbamate & $\mathrm{R}-\mathrm{OC}(=\mathrm{O}) \mathrm{NR}_{2}$ & -23.12 & $(A 68)$ & & & & & \\
\hline N-substituted carbamate & $\mathrm{R}-\mathrm{OC}(=\mathrm{O}) \mathrm{NH}-\mathrm{R}$ & 10.6 & $(A 69)$ & & & & & \\
\hline carbamate & $\mathrm{R}-\mathrm{OC}(=\mathrm{O}) \mathrm{NH}_{2}$ & {$[27.9]$} & $(A 70)$ & & & & & \\
\hline imide & $\mathrm{R}-\mathrm{C}(=\mathrm{O}) \mathrm{NHC}(=\mathrm{O})-\mathrm{R}$ & {$[7.7]$} & $(A 71)$ & & & & & \\
\hline phosphine & $\mathrm{R}_{3}-\mathrm{P}$ & {$[-20.7]$} & $(A 72)$ & & & & & \\
\hline phosphine oxide & $\mathrm{R}_{3}-\mathrm{P}=\mathrm{O}$ & {$[-32.7]$} & $(A 73)$ & & & & & \\
\hline phosphate ester & $\mathrm{P}(=\mathrm{O})(\mathrm{O}-\mathrm{R})_{4}$ & {$[-10.0]$} & $(A 74)$ & & & & & \\
\hline phosphonate ester & $\mathrm{R}-\mathrm{P}(=\mathrm{O})(\mathrm{O}-\mathrm{R})_{2}$ & {$[-14.0]$} & $(A 75)$ & & & & & \\
\hline phosphonic acid & $\mathrm{R}-\mathrm{P}=\mathrm{O}(\mathrm{OH})_{2}$ & {$[7.7]$} & $(A 76)$ & & & & & \\
\hline phosphonyl halide & $\mathrm{R}-\mathrm{P}(=\mathrm{O}) \mathrm{X}_{2}$ & {$[4.8]$} & $(A 77)$ & & & & & \\
\hline
\end{tabular}


TABLE 2. (a) Contributions of the functional group portion of the molecule-Continued

\begin{tabular}{|c|c|c|c|c|c|c|c|c|}
\hline \multirow{3}{*}{$\frac{\text { Functional groups }^{\mathrm{a}}}{\text { phosphoramidate ester }}$} & \multirow{2}{*}{\multicolumn{3}{|c|}{$\begin{array}{l}\text { Group value }\left(G_{k}\right)^{\mathrm{a}} \\
\mathrm{J} /(\mathrm{mol} \mathrm{K})\end{array}$}} & \multicolumn{5}{|c|}{$\begin{array}{c}\text { Group coefficient }\left(C_{k}\right)^{\mathrm{b}} \\
k\end{array}$} \\
\hline & & & & 2 & 3 & 4 & 5 & 6 \\
\hline & $(\mathrm{R}-\mathrm{O})_{2} \mathrm{P}(=\mathrm{O}) \mathrm{NH}-\mathrm{R}$ & {$[-0.7]$} & $(A 78)$ & & & & & \\
\hline phosphorothioate ester & $(\mathrm{R}-\mathrm{O})_{3} \mathrm{P}(=\mathrm{S})$ & 1.1 & $(A 79)$ & & & & & \\
\hline phosphorodithioate ester & $\mathrm{R}-\mathrm{S}-\mathrm{P}(=\mathrm{S})(\mathrm{O}-\mathrm{R})_{2}$ & -9.6 & $(A 80)$ & & & & & \\
\hline phosphonothioate ester & $\mathrm{R}-\mathrm{P}(=\mathrm{S})(\mathrm{O}-\mathrm{R})_{2}$ & {$[5.2]$} & $(A 81)$ & & & & & \\
\hline phosphoroamidothioate ester & $\mathrm{R}-\mathrm{NHP}(=\mathrm{S})(\mathrm{O}-\mathrm{R})_{2}$ & {$[16.0]$} & $(A 82)$ & & & & & \\
\hline phosphoroamidodithioate ester & $\mathrm{NH}_{2} \mathrm{P}(=\mathrm{S})(\mathrm{S}-\mathrm{R})(\mathrm{O}-\mathrm{R})$ & {$[6.9]$} & $(A 83)$ & & & & & \\
\hline sulfides & $\mathrm{R}-\mathrm{S}-\mathrm{R}$ & 2.1 & $(A 84)$ & & & & & \\
\hline disulfides & $\mathrm{R}-\mathrm{SS}-\mathrm{R}$ & 9.6 & $(A 85)$ & & & & & \\
\hline thiols & $\mathrm{R}-\mathrm{SH}$ & 23.0 & $(A 86)$ & & & & & \\
\hline sulfoxide & $\mathrm{R}-\mathrm{S}(\rightarrow \mathrm{O})-\mathrm{R}$ & {$[14.1]$} & $(A 87)$ & & & & & \\
\hline sulfones & $\mathrm{R}-\mathrm{S}(\rightarrow \mathrm{O})_{2}-\mathrm{R}$ & 0.3 & A88) & & & & & \\
\hline sulfonate ester & $\mathrm{R}-\mathrm{S}(\rightarrow \mathrm{O})_{2} \mathrm{O}-\mathrm{R}$ & {$[7.9]$} & $(A 89)$ & & & & & \\
\hline 1,2-disubstituted thiourea & $\mathrm{R}-\mathrm{NHC}(=\mathrm{S}) \mathrm{NH}-\mathrm{R}$ & {$[14.4]$} & $(A 90)$ & & & & & \\
\hline monosubst thiourea & $\mathrm{R}-\mathrm{NHC}(=\mathrm{S})+\mathrm{NH}_{2}$ & {$[23.1]$} & $(A 91)$ & & & & & \\
\hline thioamide & $\mathrm{R}-\mathrm{C}(=\mathrm{S}) \mathrm{NH}_{2}$ & {$[30.0]$} & $(A 92)$ & & & & & \\
\hline N,N disubstituted thiocarbamate & $\mathrm{R}-\mathrm{S}(\mathrm{C}=\mathrm{O}) \mathrm{N}-\mathrm{R}_{2}$ & {$[5.6]$} & $(A 93)$ & & & & & \\
\hline $\mathrm{N}, \mathrm{N}$-disubstituted sulfonamide & $\mathrm{R}-\mathrm{S}(\rightarrow \mathrm{O})_{2} \mathrm{~N}-\mathrm{R}_{2}$ & {$[-11.3]$} & $(A 94)$ & & & & & \\
\hline N-substituted sulfonamide & $\mathrm{R}-\mathrm{S}(\rightarrow \mathrm{O})_{2} \mathrm{NH}-\mathrm{R}$ & 6.3 & $(A 95)$ & & & & & \\
\hline sulfonic acid & $\mathrm{R}-\mathrm{S}(\rightarrow \mathrm{O})_{2} \mathrm{OH}$ & {$[1.8]$} & $(A 145)$ & & & & & \\
\hline sulfonamide & $\mathrm{R}-\mathrm{S}(\rightarrow \mathrm{O})_{2} \mathrm{NH}_{2}$ & {$[28.4]$} & (A96) & & & & & \\
\hline trisubstituted aluminum & $\mathrm{R}_{3}-\mathrm{Al}$ & {$[-24.7]$} & $(A 97)$ & & & & & \\
\hline trisubstituted arsenic & $\mathrm{R}_{3}-\mathrm{As}$ & {$[-6.5]$} & $(A 98)$ & & & & & \\
\hline trisubstituted boron & $\mathrm{R}_{3}-\mathrm{B}$ & {$[-17.2]$} & (A99) & & & & & \\
\hline trisubstituted bismuth & $\mathrm{R}_{3}-\mathrm{Bi}$ & {$[-14.5]$} & $(A 100)$ & & & & & \\
\hline trisubstituted galium & $\mathrm{R}_{3}-\mathrm{Ga}$ & {$[-11.9]$} & $(A 101)$ & & & & & \\
\hline tetrasubstituted germanium & $\mathrm{R}_{4}-\mathrm{Ge}$ & {$[-35.2]$} & $(A 102)$ & & & & & \\
\hline disubstituted germanium & $\mathrm{R}_{2} \mathrm{GeH}_{2}$ & {$[-14.7]$} & $(A 103)$ & & & & & \\
\hline disubstituted mercury & $\mathrm{R}_{2}-\mathrm{Hg}$ & {$[8.4]$} & $(A 104)$ & & & & & \\
\hline trisubstituted indium & $\mathrm{R}_{3}-\mathrm{In}$ & {$[-19.3]$} & $(A 105)$ & & & & & \\
\hline tetrasubstituted lead & $\mathrm{R}_{4}-\mathrm{Pb}$ & {$[-30.2]$} & $(A 106)$ & & & & & \\
\hline trisubstituted antimony & $\mathrm{R}_{3}-\mathrm{Sb}$ & {$[-12.7]$} & $(A 107)$ & & & & & \\
\hline disubstituted selenium & $\mathrm{R}_{2}-\mathrm{Se}$ & {$[6.0]$} & $(A 108)$ & & & & & \\
\hline quaternary silicon & $\mathrm{R}_{4}-\mathrm{Si}$ & -27.1 & (A109) & & & & & \\
\hline quaternary tin & $\mathrm{R}_{4}-\mathrm{Sn}$ & -24.2 & $(A 110)$ & & & & & \\
\hline disubstituted zinc & $\mathrm{R}_{2}-\mathrm{Zn}$ & {$[11.1]$} & $(A 111)$ & & & & & \\
\hline disubstituted telluride & $\mathrm{R}_{2}-\mathrm{Te}$ & {$[-2.2]$} & $(A 140)$ & & & & & \\
\hline trisubstituted germanium & $\mathrm{R}_{3}-\mathrm{GeH}$ & {$[-27.8]$} & $(A 141)$ & & & & & \\
\hline disubstituted arsinic acid & $\mathrm{R}_{2}-\mathrm{AsO}_{2} \mathrm{H}$ & {$[-24]$} & $(A 142)$ & & & & & \\
\hline trisubstituted thallium & $\mathrm{R}_{3}-\mathrm{Th}$ & {$[1]$} & $(A 143)$ & & & & & \\
\hline disubstituted cadmium & $\mathrm{R}_{2}-\mathrm{Cd}$ & {$[-2]$} & $(A 144)$ & & & & & \\
\hline
\end{tabular}

${ }^{\mathrm{a}} \mathrm{R}$ : any alkyl or aryl group unless specified otherwise; $\mathrm{X}$ : any halogen; units: $\mathrm{J}_{\mathrm{mol}}^{-1} \mathrm{~K}^{-1}$.

${ }^{\mathrm{b}}$ Unassigned values beneath each of the group coefficients; $C_{k}$ can be assumed to be 1 . 
TABLE 2. (b) Contributions of functional groups as part of a ring

\begin{tabular}{|c|c|c|c|}
\hline \multicolumn{2}{|l|}{$\begin{array}{l}\text { Heteroatoms and functional groups } \\
\text { comprising a portion of a ring }\end{array}$} & \multicolumn{2}{|c|}{ Group value $\left(G_{k}\right)^{\mathrm{a}}$} \\
\hline cyclic ether & $R-\mathrm{O}-R$ & 1.2 & $(A 112)$ \\
\hline cyclic peroxide & $R-\mathrm{OO}-R$ & {$[27.7]$} & $(A 113)$ \\
\hline cyclic ketone & $R-C(=O)-R$ & -1.4 & $(A 114)$ \\
\hline cyclic ester & $R-C(=O) O-R$ & 3.1 & $(A 115)$ \\
\hline cyclic carbonate & $R-O C(=O) O-R$ & {$[1.3]$} & $(A 116)$ \\
\hline cyclic anhydride & $R-C(=O)-O-C(=O)-R$ & 2.3 & $(A 117)$ \\
\hline cyclic $s p^{2}$ nitrogen & $R=\mathrm{N}-R$ & 0.5 & $(A 118)$ \\
\hline cyclic tertiary amine & $R_{2}>\mathrm{N}-\mathrm{R}$ & -19.3 & $(A 119)$ \\
\hline cyclic tertiary amine-N-nitro, & $R_{2}>\mathrm{N}-\left(\mathrm{NO}_{2}\right)-\mathrm{R}$ & -27.1 & $(A 120)$ \\
\hline cyclic tertiary amine-N-nitroso & $R_{2}>\mathrm{N}-(\mathrm{N}=\mathrm{O})-\mathrm{R}$ & -27.1 & $(A 120)$ \\
\hline cyclic secondary amine & $R_{2}>\mathrm{NH}$ & 2.2 & $(A 121)$ \\
\hline cyclic tertiary amine $\mathrm{N}$-oxide & $R_{2}>\mathrm{N}(\rightarrow \mathrm{O})-\mathrm{R}$ & {$[-22.2]$} & $(A 122)$ \\
\hline cyclic azoxy group & $R=\mathrm{N}(\rightarrow \mathrm{O})-R$ & {$[2.9]$} & $(A 123)$ \\
\hline cyclic sec amide & $R-\mathrm{C}(=\mathrm{O}) \mathrm{NH}-R$ & 2.7 & $(A 124)$ \\
\hline cyclic tertiary amide & $R-\mathrm{C}(=\mathrm{O}) \mathrm{N}<\mathrm{R} R$ & -21.7 & $(A 125)$ \\
\hline cyclic tertiary amide & $\mathrm{R}-\mathrm{C}(=O) N<R_{2}$ & {$[-9]$} & $(A 146)$ \\
\hline cyclic carbamate & $R-\mathrm{OC}(=\mathrm{O}) \mathrm{N}-\mathrm{R} R$ & {$[-5.2]$} & $(A 126)$ \\
\hline cyclic carbamate & $R-\mathrm{OC}(=\mathrm{O}) \mathrm{N}-\mathrm{H} R$ & {$[19.7]$} & $(A 125)$ \\
\hline cyclic urea & $R-\mathrm{NC}(=\mathrm{O}) \mathrm{N}<\mathrm{R} R$ & {$[-40.6]$} & $(A 127)$ \\
\hline N-substituted cyclic imide & $R-\mathrm{C}(=\mathrm{O}) \mathrm{N}(\mathrm{R}) \mathrm{C}(=\mathrm{O})-R$ & {$[1.1]$} & $(A 128)$ \\
\hline cyclic imide & $R-\mathrm{C}(=\mathrm{O}) \mathrm{N}(\mathrm{H}) \mathrm{C}(=\mathrm{O})-R$ & {$[1.4]$} & $(A 129)$ \\
\hline cyclic phosphorothioate & $R-\mathrm{O}-\mathrm{P}(=\mathrm{S})<(\mathrm{OR})(\mathrm{O} R)$ & {$[-15.6]$} & $(A 130)$ \\
\hline cyclic sulfide & $R-\mathrm{S}-R$ & 2.9 & $(A 131)$ \\
\hline cyclic disulfide & $R-\mathrm{SS}-R$ & {$[-6.4]$} & $(A 132)$ \\
\hline cyclic disulfide S-oxide & $R-\mathrm{SS}(\rightarrow \mathrm{O})-R$ & [1.9] & $(A 133)$ \\
\hline cyclic sulphone & $R-\mathrm{S}(\rightarrow \mathrm{O})_{2}-R$ & {$[-10.4]$} & $(A 134)$ \\
\hline cyclic thiocarbonate & $R-\mathrm{OC}(=\mathrm{O}) \mathrm{S}-R$ & {$[14.2]$} & $(A 135)$ \\
\hline cyclic sulfate & $R-\mathrm{OS}(\rightarrow \mathrm{O})_{2} \mathrm{O}-R$ & 0.9 & $(A 136)$ \\
\hline cyclic N-substituted sulphonamide & $R-\mathrm{S}(\rightarrow \mathrm{O})_{2} \mathrm{NH}-R$ & {$[-0.4]$} & $(A 137)$ \\
\hline cyclic thiocarbamate & $R-\mathrm{S}-(\mathrm{C}=\mathrm{O}) \mathrm{NH} R$ & {$[13.9]$} & $(A 138)$ \\
\hline cyclic quaternary silicon & $\mathrm{R}_{2}>\mathrm{Si}<R_{2}$ & -34.7 & $(A 139)$ \\
\hline
\end{tabular}

${ }^{\mathrm{a}} \mathrm{R}$ : any alkyl or aryl group unless specified otherwise; values in brackets are tentative assignments; units: $\mathrm{J}_{\text {mol }}{ }^{-1} \mathrm{~K}^{-1}$.

${ }^{\mathrm{b}}$ The $R$ groups that are a part of the ring structure are designated by italics. 
TABLE 3. Estimations of total phase change entropies and enthalpies of hydrocarbons $\mathrm{s}^{\mathrm{a}}$

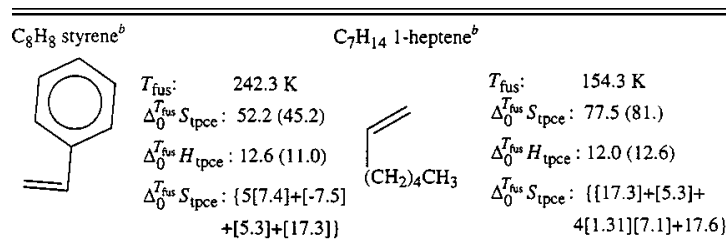
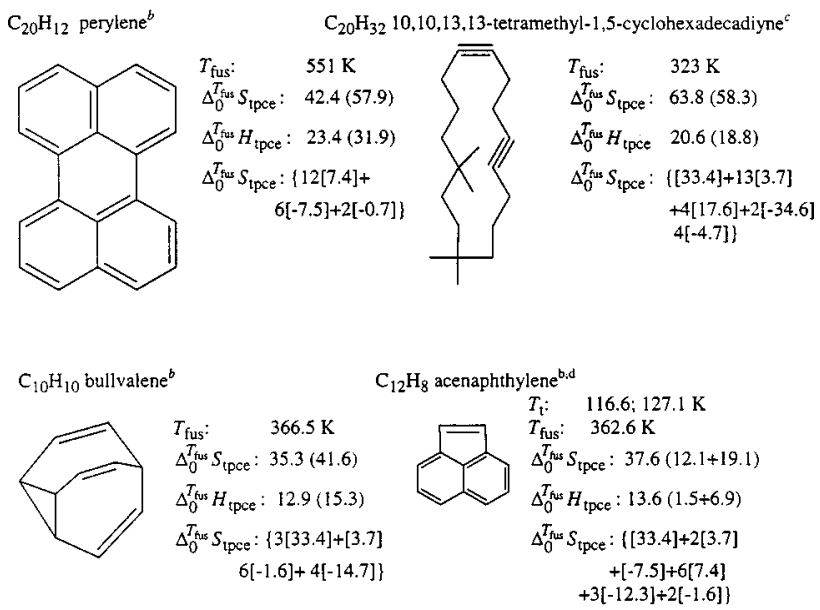

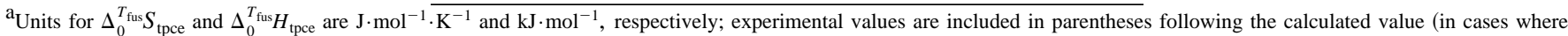
additional solid-solid transitions are involved, the first term given is the total property associated with the transition(s) and the second term represents the fusion property).

${ }^{\mathrm{b}}$ Reference 11.

${ }^{\mathrm{c}}$ Reference 12

$\mathrm{d}_{\text {Reference } 13}$

TABLE 4. Estimations of total phase change entropies and enthalpies

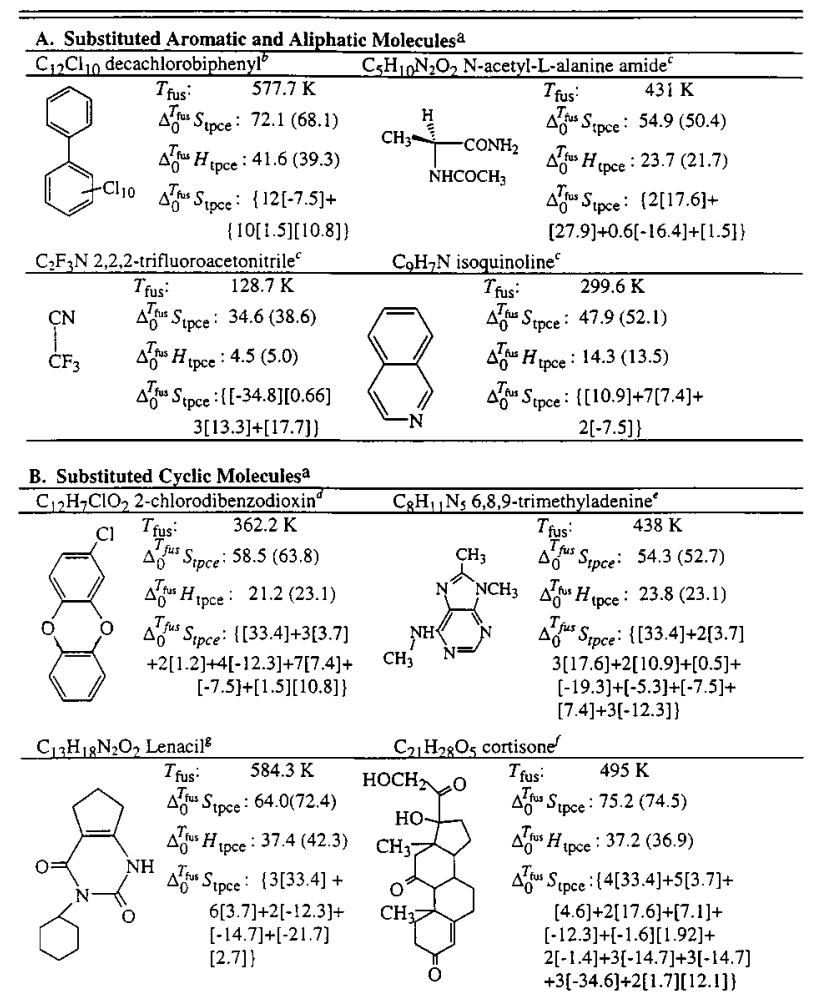

${ }^{\mathrm{a}}$ Units for $\Delta_{0}^{T_{\text {fus }}} S_{\text {tpce }}$ and $\Delta_{0}^{T_{\text {fus }}} H_{\text {tpce }}$ are $\mathrm{J} \cdot \mathrm{mol}^{-1} \cdot \overline{\mathrm{K}^{-1}}$ and $\mathrm{kJ} \cdot \mathrm{mol}^{-1}$, respectively; experimental values are given in parentheses.

$\mathrm{b}_{\text {Reference } 14 .}$

${ }^{\mathrm{c}}$ Reference 11.

${ }^{\mathrm{d}}$ Reference 15

$\mathrm{e}_{\text {Reference } 16 .}$

$\mathrm{f}_{\text {Reference } 17 .}$

$\mathrm{g}_{\text {Reference } 18}$ 
TABLE 5. Experimental and calculated total phase change enthalpy and entropy of database ${ }^{\mathrm{a}}$

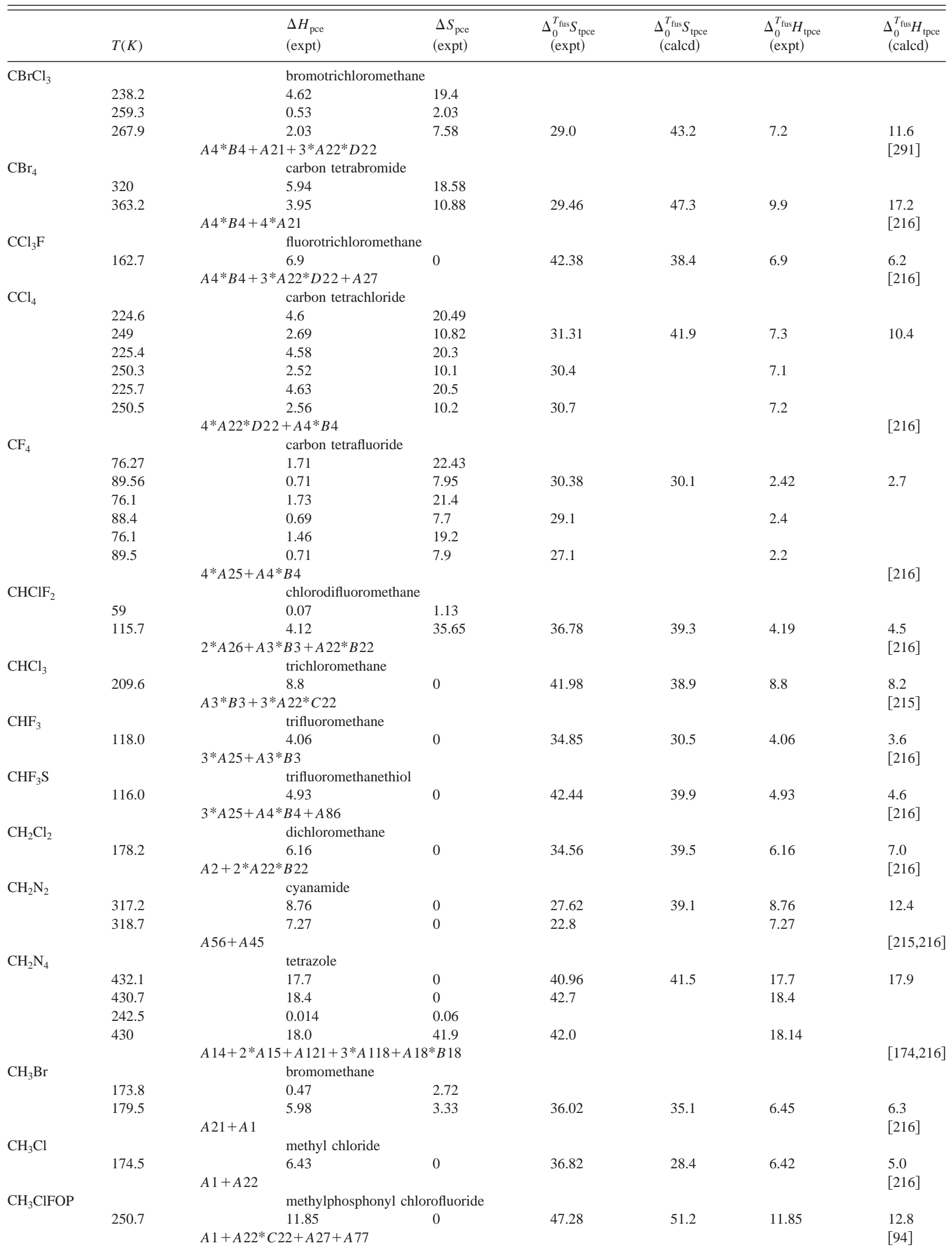


PHASE CHANGE ENTHALPIES AND ENTROPIES

Table 5. Experimental and calculated total phase change enthalpy and entropy of database-Continued

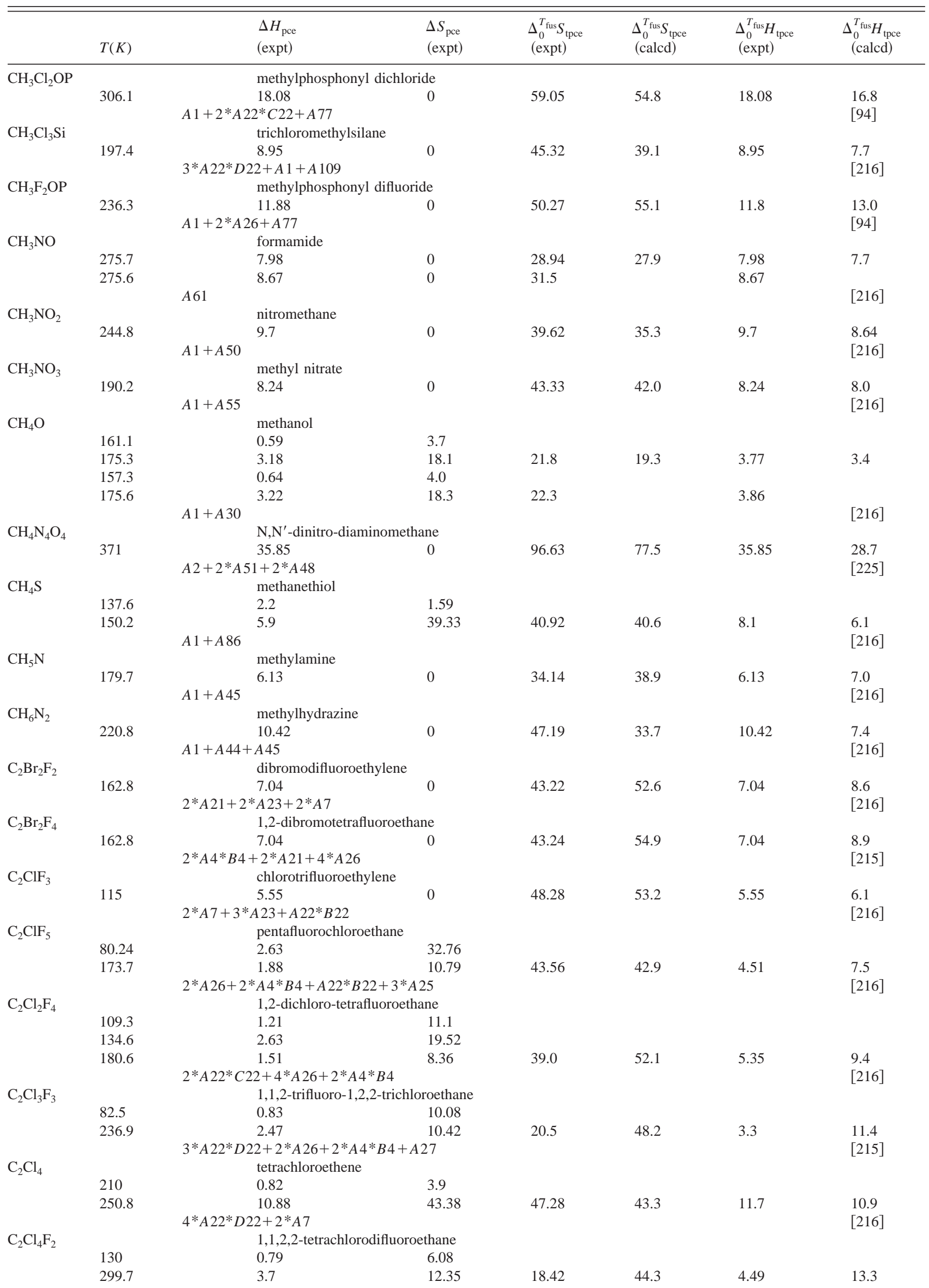


Table 5. Experimental and calculated total phase change enthalpy and entropy of database-Continued

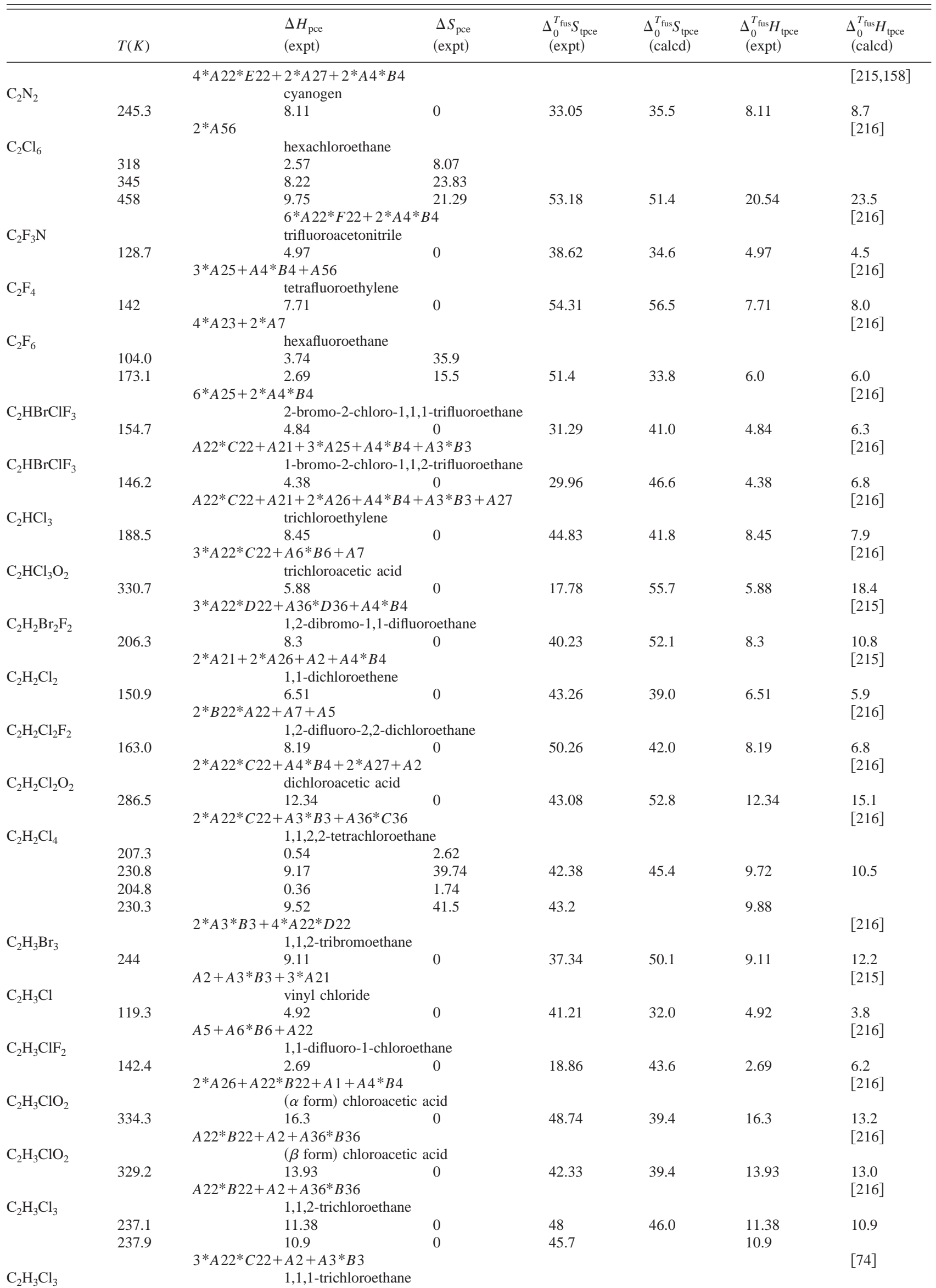


Table 5. Experimental and calculated total phase change enthalpy and entropy of database-Continued

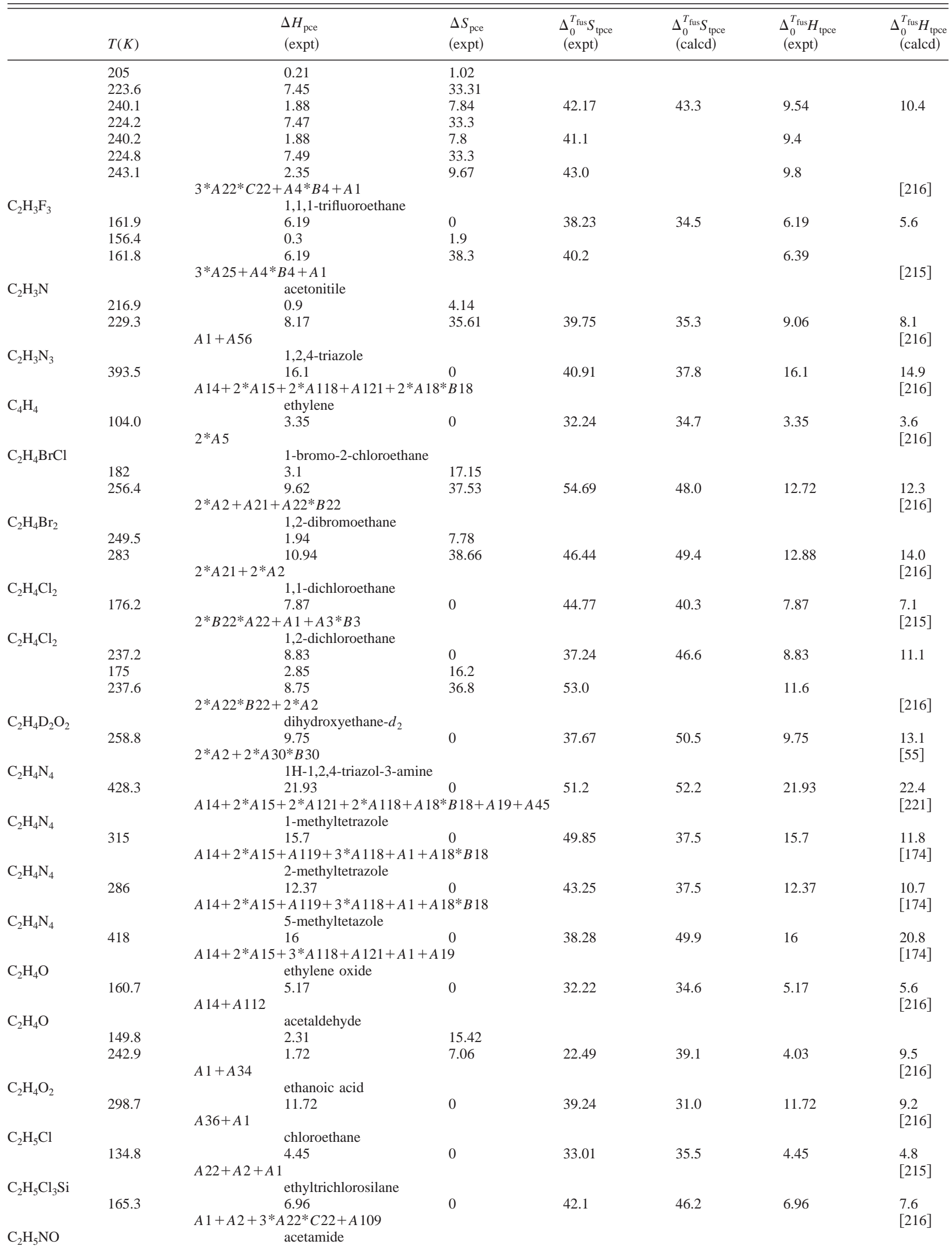


Table 5. Experimental and calculated total phase change enthalpy and entropy of database-Continued

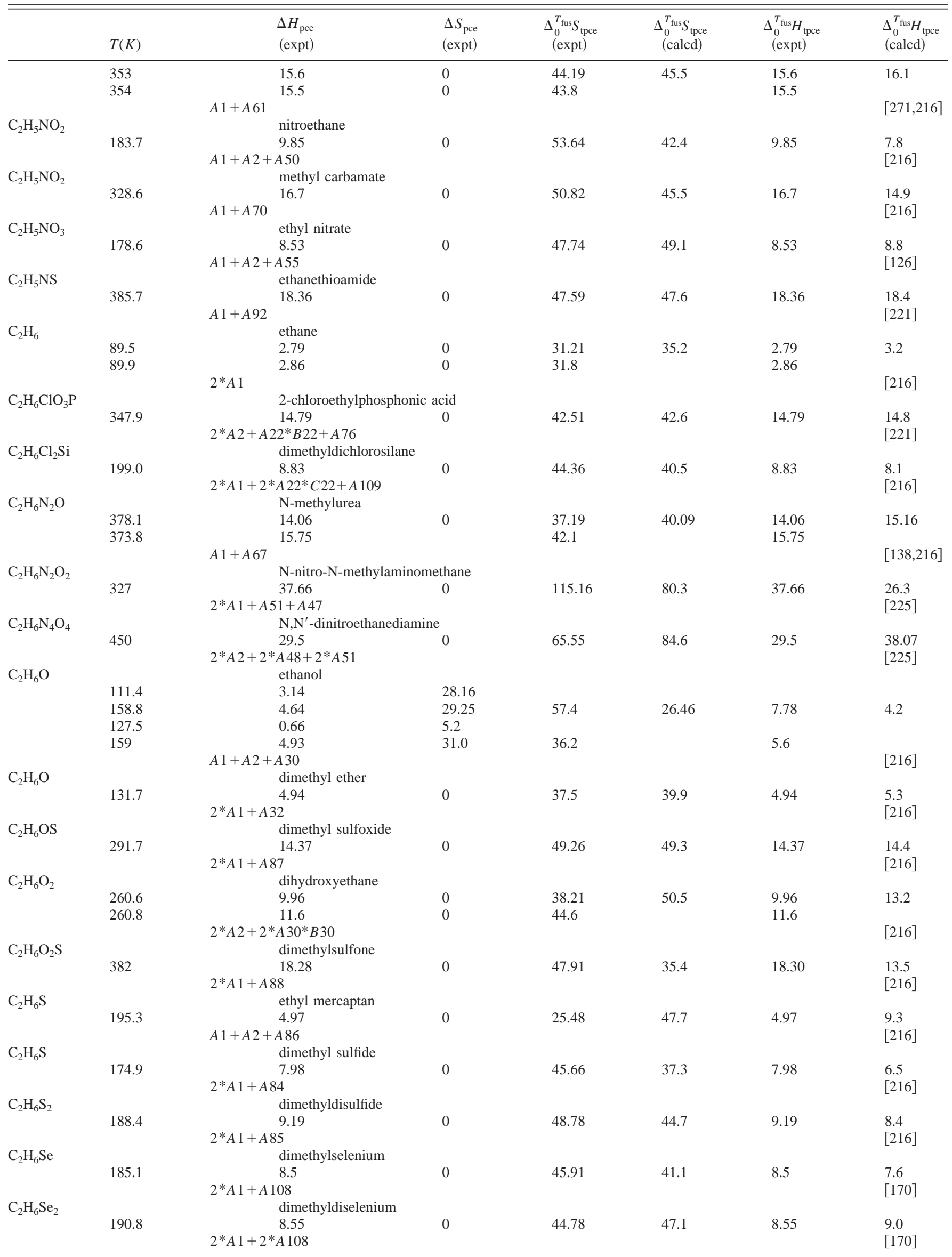


Table 5. Experimental and calculated total phase change enthalpy and entropy of database-Continued

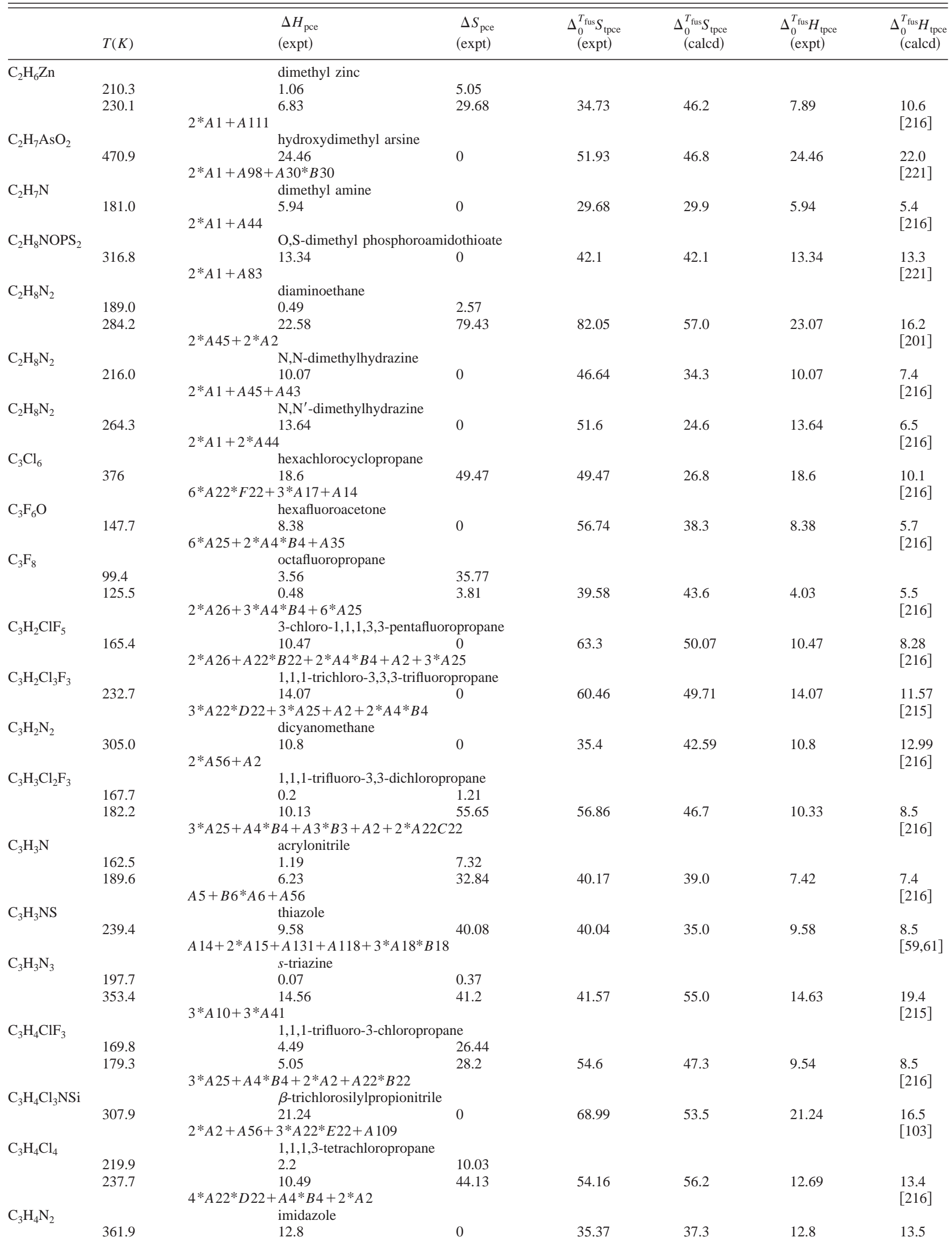


Table 5. Experimental and calculated total phase change enthalpy and entropy of database-Continued

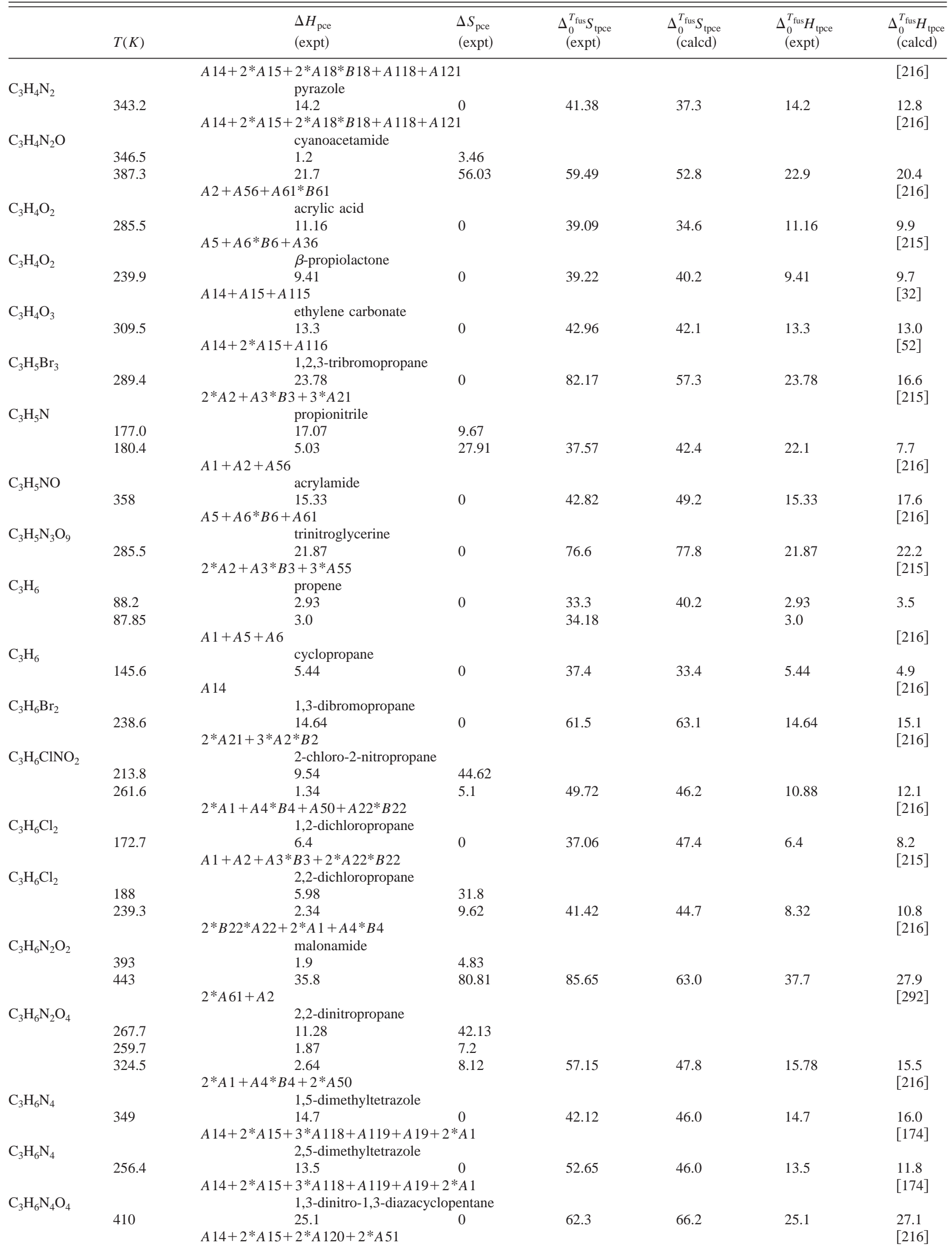


Table 5. Experimental and calculated total phase change enthalpy and entropy of database-Continued

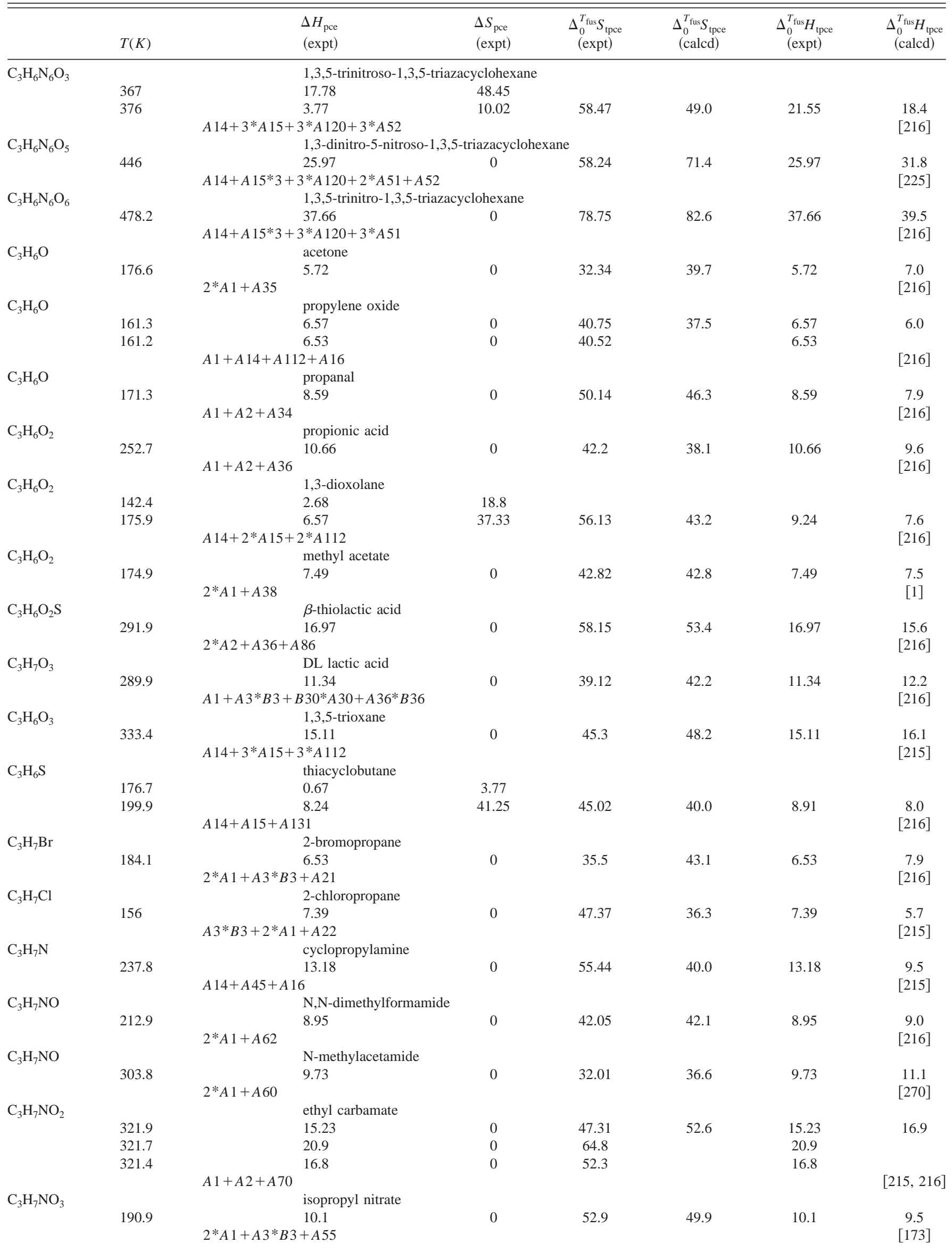


Table 5. Experimental and calculated total phase change enthalpy and entropy of database-Continued

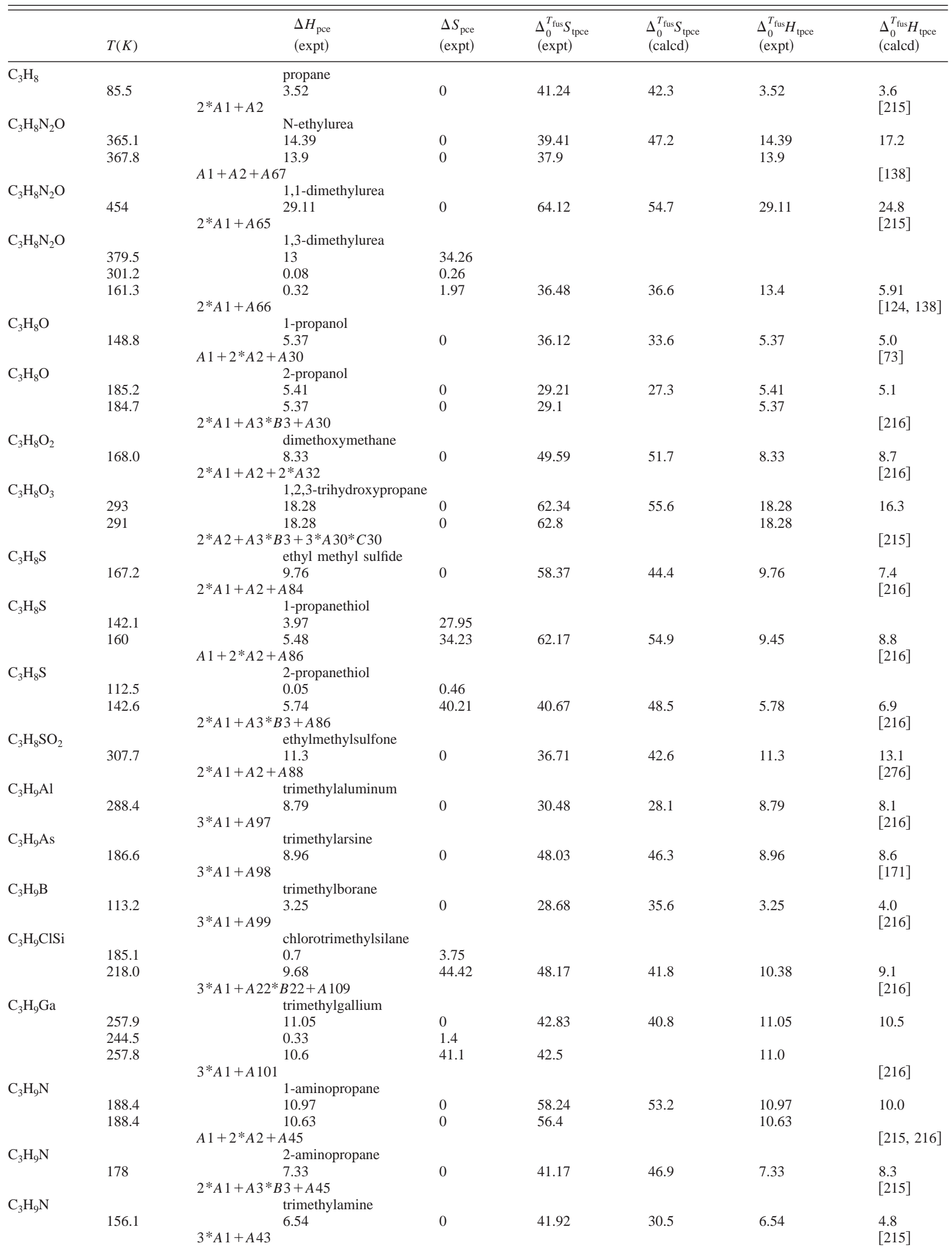


Table 5. Experimental and calculated total phase change enthalpy and entropy of database-Continued

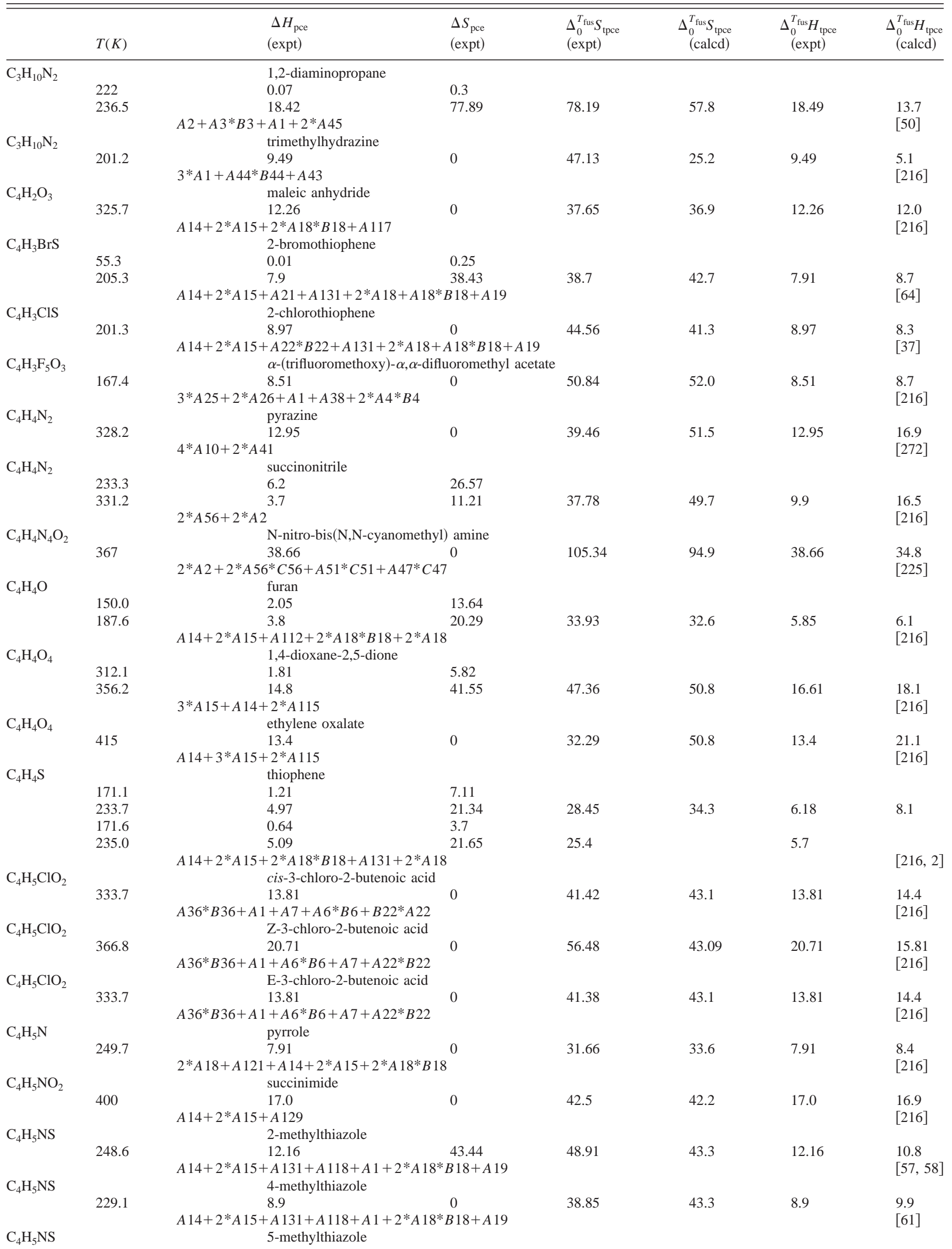


Table 5. Experimental and calculated total phase change enthalpy and entropy of database-Continued

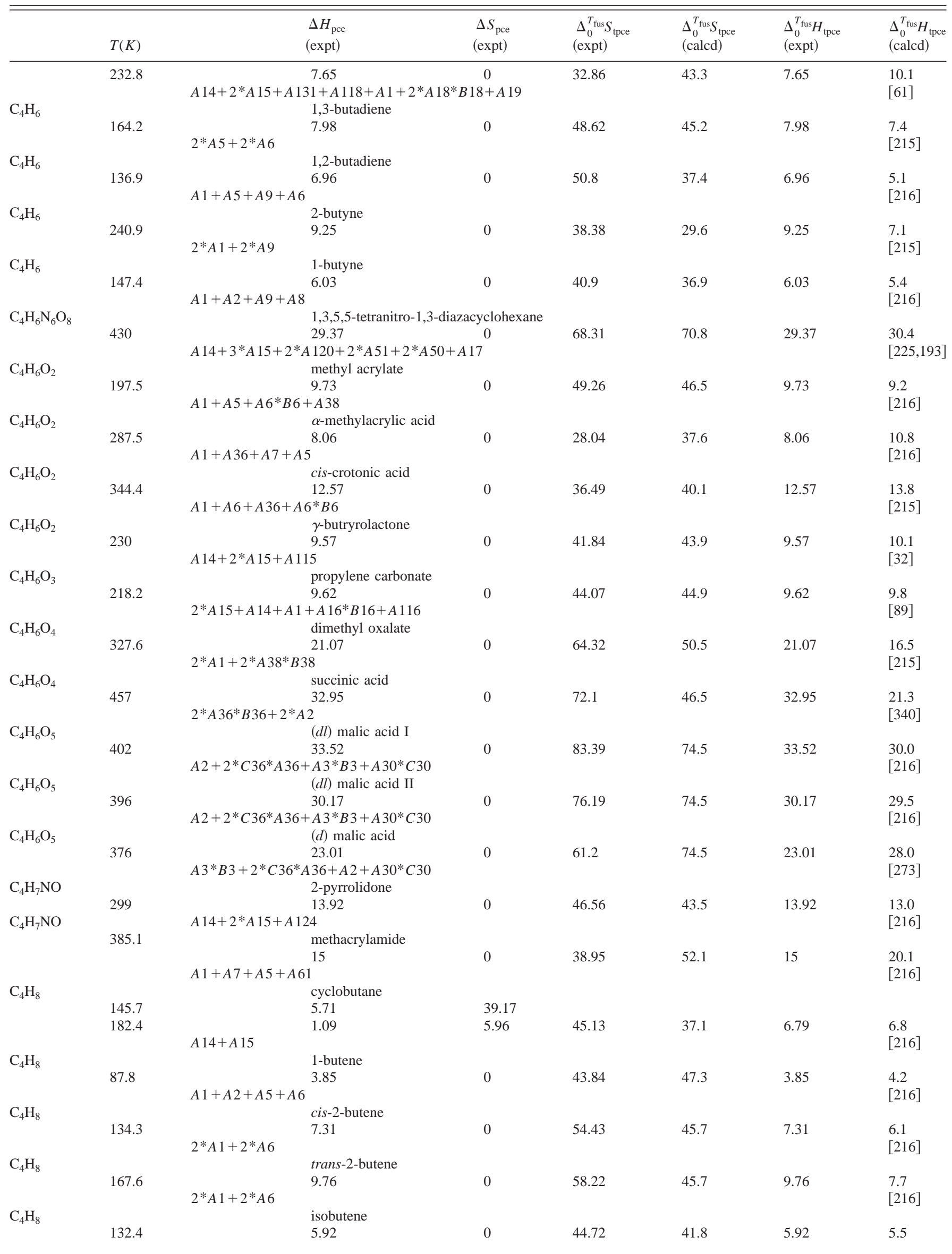


Table 5. Experimental and calculated total phase change enthalpy and entropy of database-Continued

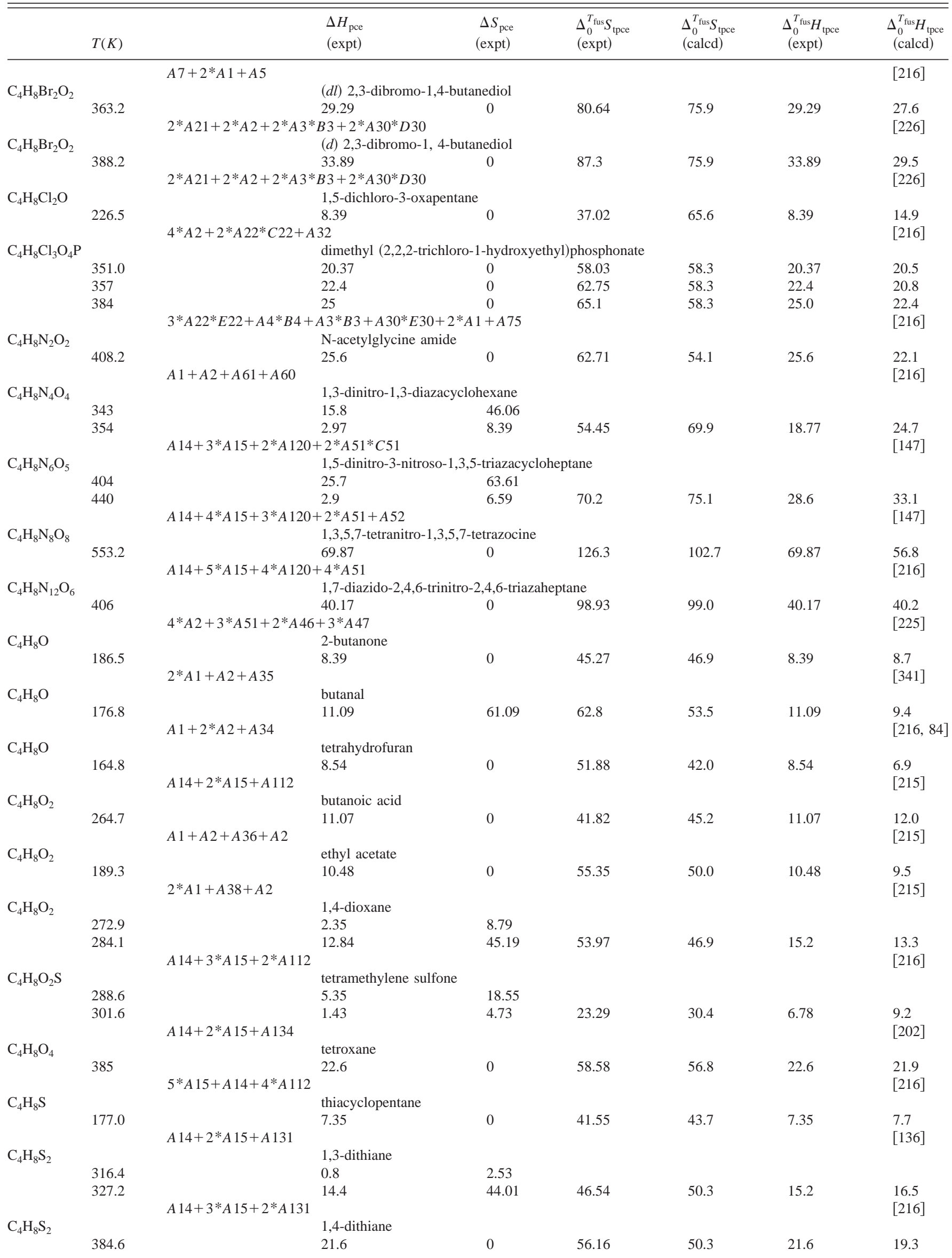


Table 5. Experimental and calculated total phase change enthalpy and entropy of database-Continued

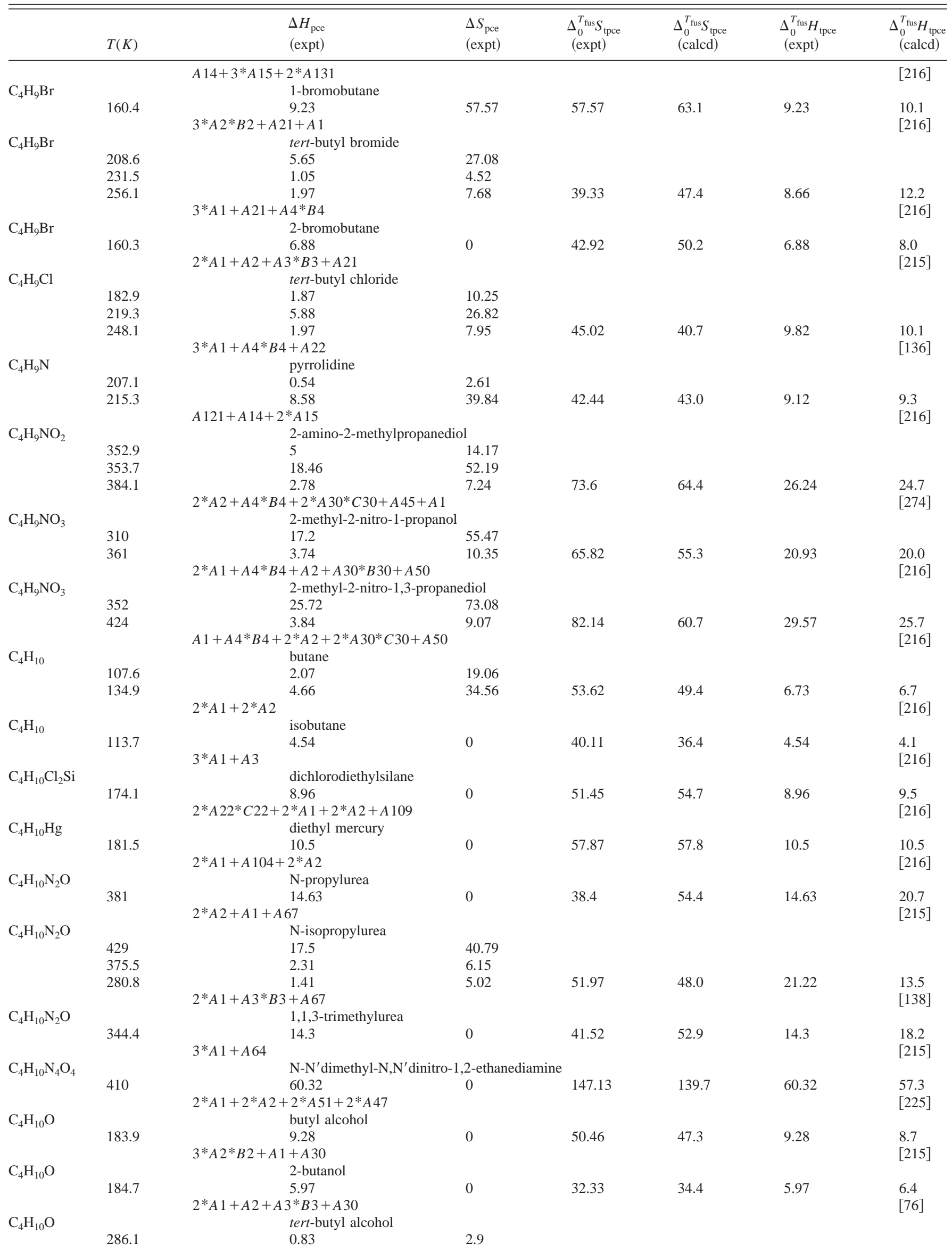


Table 5. Experimental and calculated total phase change enthalpy and entropy of database-Continued

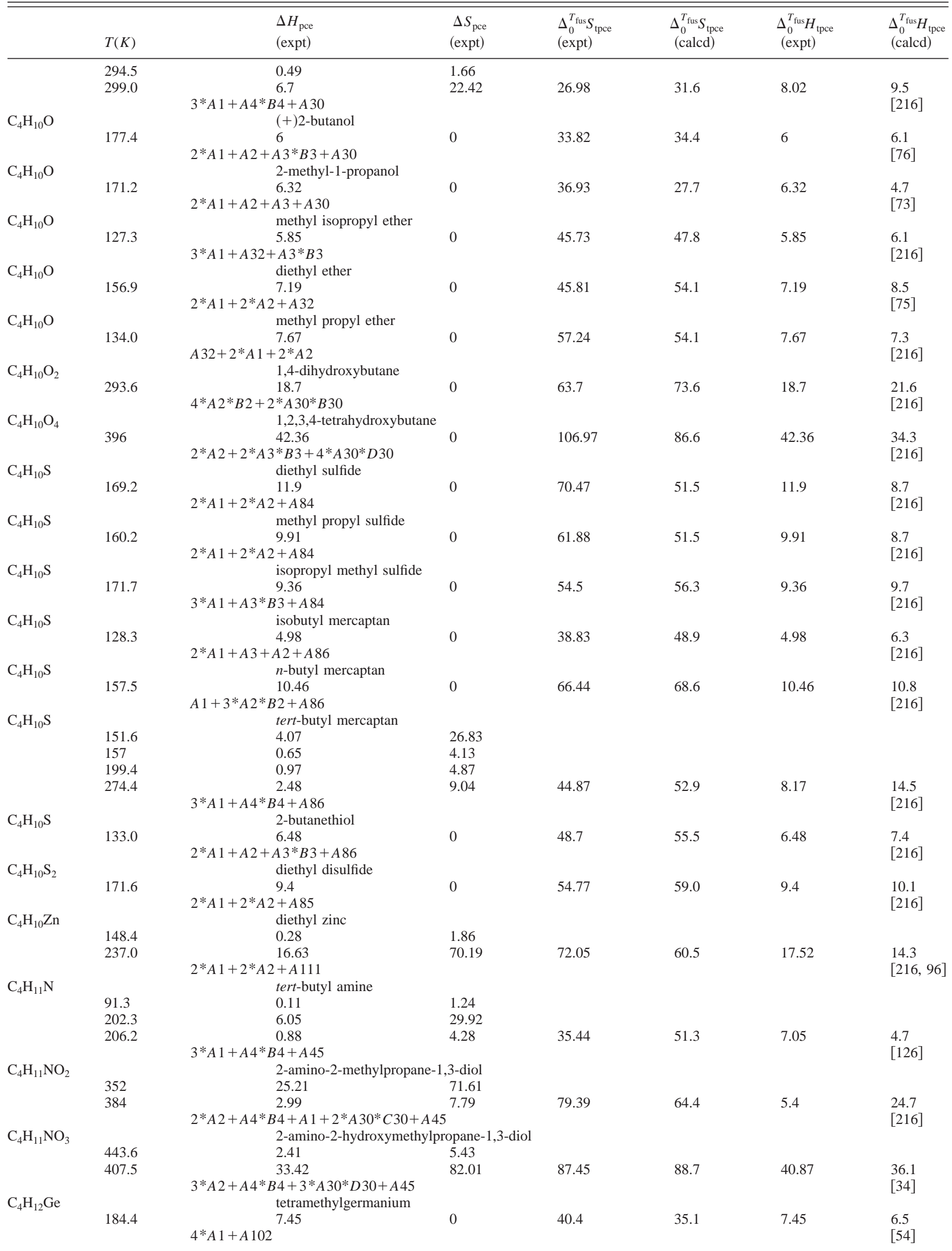


Table 5. Experimental and calculated total phase change enthalpy and entropy of database-Continued

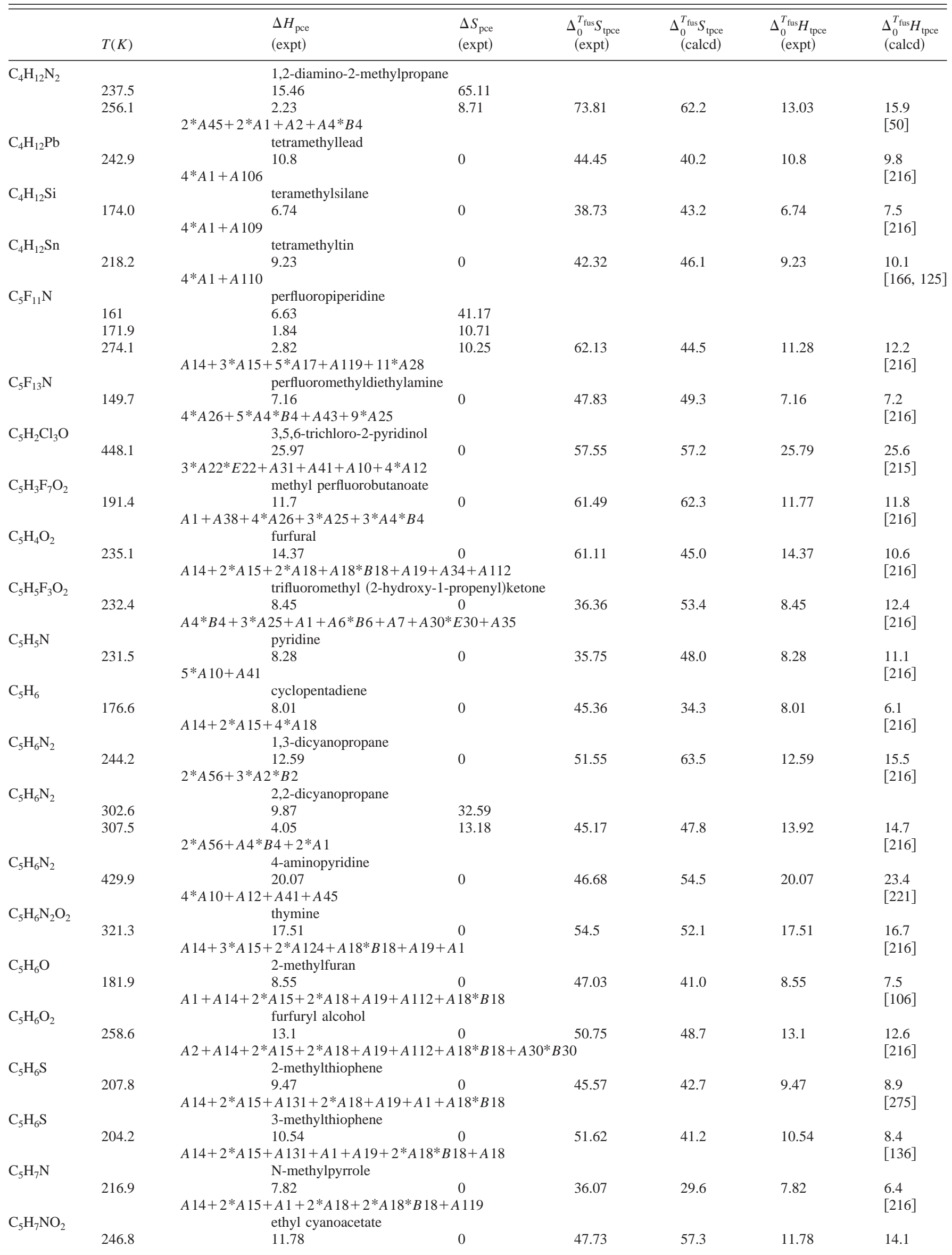


Table 5. Experimental and calculated total phase change enthalpy and entropy of database-Continued

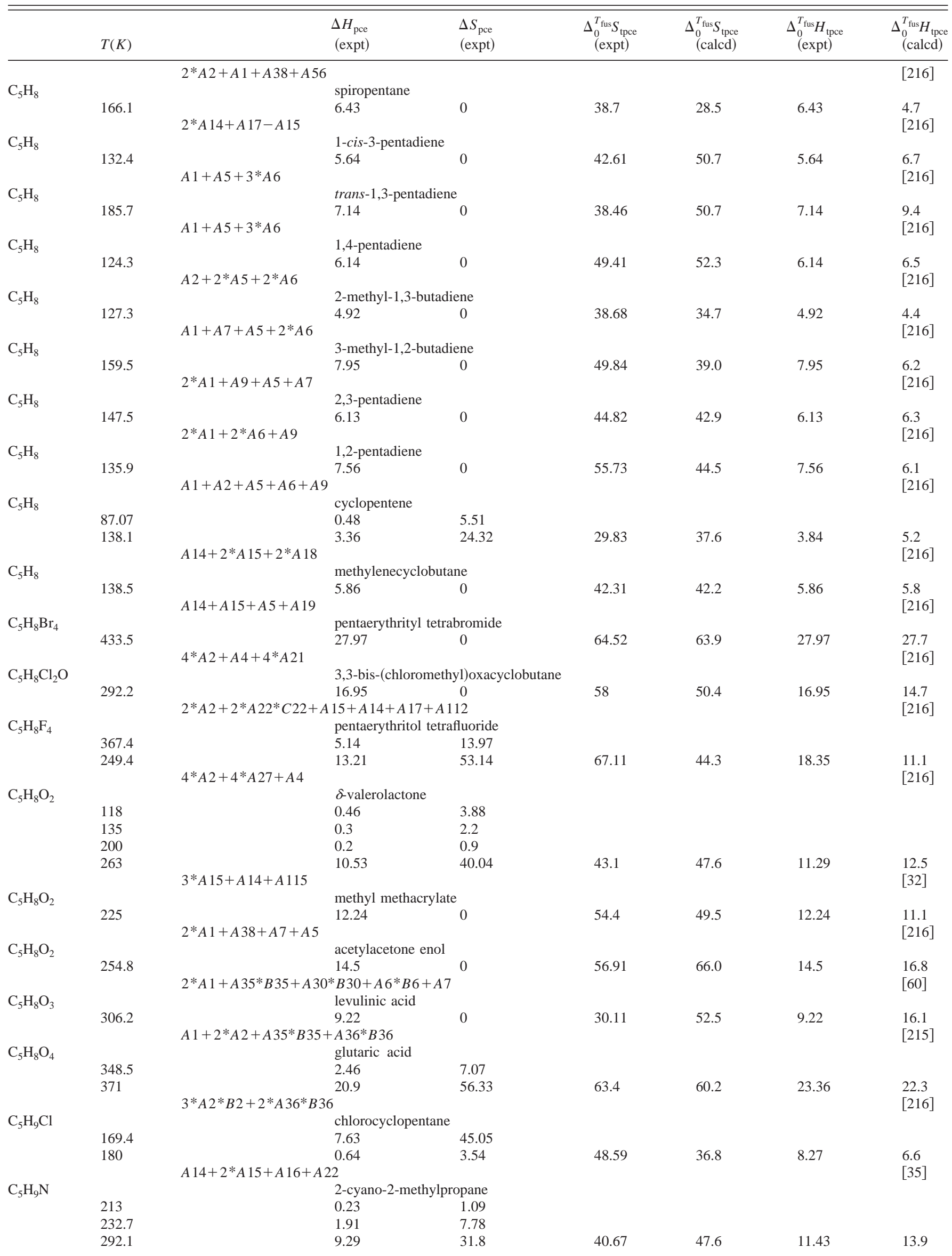


Table 5. Experimental and calculated total phase change enthalpy and entropy of database-Continued

\begin{tabular}{|c|c|c|c|c|c|c|c|}
\hline & $T(K)$ & $\begin{array}{l}\Delta H_{\text {pce }} \\
\text { (expt) }\end{array}$ & $\begin{array}{l}\Delta S_{\text {pce }} \\
\text { (expt) }\end{array}$ & $\begin{array}{c}\Delta_{0}^{T_{\text {fus }}} S_{\text {tpce }} \\
(\text { expt })\end{array}$ & $\begin{array}{c}\Delta_{\text {fus }}^{T_{\text {tus }}} S_{\text {pce }} \\
\text { (calcd) }\end{array}$ & $\begin{array}{c}\Delta_{\text {fus }}^{T_{\text {tus }}} H_{\text {tpe }} \\
(\text { expt })\end{array}$ & $\begin{array}{c}\Delta_{0}^{T_{\text {fus }}} H_{\text {tpce }} \\
\text { (calcd) }\end{array}$ \\
\hline \multirow{3}{*}{$\mathrm{C}_{5} \mathrm{H}_{10} \mathrm{ClNO}$} & & $3 * A 1+A 4 * B 4+A 56$ & & & & & {$[216]$} \\
\hline & & 2-chloro-N-isopropylacetan & & & & & \\
\hline & 351.3 & $\begin{array}{r}26.05 \\
2 * 05\end{array}$ & 0 & 74.15 & 50.1 & 26.05 & 17.6 \\
\hline \multirow{3}{*}{$\mathrm{C}_{5} \mathrm{H}_{9} \mathrm{NO}$} & & $\begin{array}{c}2 * A 1+A 2+A 3 * B 3+A 60+A 22 * B 22 \\
\text { 2-piperidone }\end{array}$ & & & & & \\
\hline & 342.3 & 16.1 & 0 & 47.02 & 47.2 & 16.1 & 16.2 \\
\hline & & $A 14+3 * A 15+A 124$ & & & & & [227] \\
\hline \multirow{4}{*}{$\mathrm{C}_{5} \mathrm{H}_{10}$} & & cyclopentane & & & & & \\
\hline & 122 & 4.9 & 40.13 & & & & \\
\hline & 138 & 0.34 & 2.49 & & & & \\
\hline & 179.7 & $A 14+2 * A 15^{0.6}$ & 3.35 & 45.96 & 40.8 & 5.84 & $\begin{array}{l}7.3 \\
{[216]}\end{array}$ \\
\hline \multirow{3}{*}{$\mathrm{C}_{5} \mathrm{H}_{10}$} & & cis-2-pentene & & & & & \\
\hline & 121.8 & 7.11 & 0 & 58.39 & 52.8 & 7.11 & 6.4 \\
\hline & & $2 * A 1+A 2+2 * A 6$ & & & & & [215] \\
\hline \multirow{2}{*}{$\mathrm{C}_{5} \mathrm{H}_{10}$} & & trans-2-pentene & & & & & \\
\hline & 133.0 & $\begin{array}{r}8.35 \\
2 * A 1+A 2+2 * A 6\end{array}$ & 0 & 62.82 & 52.8 & 8.35 & $\begin{array}{l}7.0 \\
{[215]}\end{array}$ \\
\hline \multirow[t]{2}{*}{$\mathrm{C}_{5} \mathrm{H}_{10}$} & & 1-pentene & & & & & \\
\hline & 107.9 & 5.81 & 55 & 53.82 & 54.4 & 5.81 & 5.9 \\
\hline \multirow{3}{*}{$\mathrm{C}_{5} \mathrm{H}_{10}$} & & $A 1+2 * A 2+A 5+A 6$ & & & & & [215] \\
\hline & 104.7 & $\begin{array}{l}\text { 3-methyl-1-butene } \\
5.36\end{array}$ & 0 & 51.19 & 41.4 & 5.36 & \\
\hline & & $2 * A 1+A 3+A 5+A 6$ & & 1.1. & & & [216] \\
\hline \multirow[t]{3}{*}{$\mathrm{C}_{5} \mathrm{H}_{10}$} & & 2-methyl-2-butene & & & & & \\
\hline & 139.4 & 7.60 & 0 & 54.47 & 59.4 & 7.60 & 8.3 \\
\hline & & $3 * A 1+A 7+A 5$ & & & & & [216] \\
\hline \multirow{2}{*}{$\mathrm{C}_{5} \mathrm{H}_{10}$} & & 2-methyl-1-butene & & & & & \\
\hline & 135.6 & 7.91 & 0 & 58.34 & 48.9 & 7.91 & 6.6 \\
\hline \multirow[t]{3}{*}{$\mathrm{C}_{5} \mathrm{H}_{10}$} & & $\begin{array}{c}2 * A 1+A 2+A 7+A 5 \\
\text { methylcyclobutane }\end{array}$ & & & & & [216] \\
\hline & 138.6 & 5.76 & 0 & 41.56 & 39.9 & 5.76 & 5.5 \\
\hline & & $\begin{array}{c}A 1+A 14+A 15+A 16 \\
\text { N-acetyl-L-alanine amide }\end{array}$ & & & & & [215] \\
\hline \multirow{2}{*}{$\mathrm{C}_{5} \mathrm{H}_{10} \mathrm{~N}_{2} \mathrm{O}_{2}$} & 431 & 21.7 & 0 & 50.35 & 54.9 & 21.7 & \\
\hline & & $2 * A 1+A 3 * B 3+A 61+A 60$ & & & & & {$[216]$} \\
\hline \multirow{3}{*}{$\mathrm{C}_{5} \mathrm{H}_{10} \mathrm{~N}_{2} \mathrm{O}_{2} \mathrm{~S}$} & & 5-methyl N-(methylcarbam & yloxy)th & & & & \\
\hline & 352.7 & 21.73 & 0 & 61.61 & 53.0 & 21.73 & \\
\hline & & $3 * A 1+A 69+A 42+A 7+A 84$ & & & & & [221] \\
\hline \multirow[t]{2}{*}{$\mathrm{C}_{5} \mathrm{H}_{10} \mathrm{~N}_{2} \mathrm{O}_{3}$} & & alanylglycine (with decomp & & & & & \\
\hline & 508.0 & $\begin{array}{c}56.6 \\
A 1+A 2+A 3 * B 3+A 45+A 36 * C 36+A 66\end{array}$ & 0 & 111.43 & 67.7 & 56.6 & $\begin{array}{l}34.4 \\
{[216]}\end{array}$ \\
\hline \multirow[t]{4}{*}{$\mathrm{C}_{5} \mathrm{H}_{10} \mathrm{~N}_{4} \mathrm{O}_{4}$} & & 1,3-dinitro-1,3-diazacycloh & ptane & & & & \\
\hline & 369 & 21.8 & 59.08 & & & & \\
\hline & 374 & 2.8 & 7.49 & 66.57 & 73.6 & 24.6 & 27.5 \\
\hline & & $A 14+4 * A 15+2 * A 120+2 * A 51$ & & & & & [147] \\
\hline \multirow{4}{*}{$\mathrm{C}_{5} \mathrm{H}_{10} \mathrm{O}$} & & pivaldehyde & & & & & \\
\hline & 158.5 & 0.5 & 3.15 & & & & \\
\hline & 183.9 & 4.81 & 26.15 & & & & \\
\hline & 272.1 & 2.52 & 9.26 & 38.56 & 51.4 & 7.83 & 14.0 \\
\hline & & $3 * A 1+A 4 * B 4+A 34$ & & & & & [163] \\
\hline $\mathrm{C}_{5} \mathrm{H}_{10} \mathrm{O}$ & & 3-pentanone & & & & & \\
\hline & 118.5 & 0.11 & 0.96 & & & & \\
\hline & 180 & 0.01 & 0.04 & & & & \\
\hline & 234.2 & 11.59 & 49.5 & 50.5 & 54.0 & 11.71 & 12.7 \\
\hline & & $2 * A 1+2 * A 2+A 35$ & & & & & [341] \\
\hline $\mathrm{C}_{5} \mathrm{H}_{10} \mathrm{O}$ & & 2-pentanone & & & & & \\
\hline & 110 & 2.09 & 2.18 & & & & \\
\hline & 196.3 & $\begin{array}{r}10.63 \\
2 * A 1+2 * A 2+A 35\end{array}$ & 54.14 & 56.32 & 54.0 & 12.72 & 10.6 \\
\hline $\mathrm{C}_{5} \mathrm{H}_{10} \mathrm{O}$ & & cyclopentanol & & & & & \\
\hline & 202.8 & 3.71 & 18.28 & & & & \\
\hline & 257.4 & 1.54 & 5.98 & 24.27 & 27.8 & 5.24 & 7.2 \\
\hline $\mathrm{C}_{5} \mathrm{H}_{1} \mathrm{O}$ & & $A 14+2 * A 15+A 16+A 30$ & & & & & [216] \\
\hline${ }_{5} \mathrm{H}_{10} \mathrm{U}$ & 180.0 & $\begin{array}{l}\text { isopropyl methyl ketone } \\
9.34\end{array}$ & 0 & 519 & 47.5 & 9.34 & \\
\hline & & $3 * A 1+A 3 * B 3+A 35$ & & & & & {$[216]$} \\
\hline $\mathrm{C}_{5} \mathrm{H}_{10} \mathrm{O}_{2}$ & 239.5 & $\begin{array}{l}\text { pentanoic acid } \\
14.16\end{array}$ & 0 & 59.14 & 58.9 & 14.16 & 14.1 \\
\hline & & $A 1+3 * A 2 * B 2+A 36$ & & & & & {$[216]$} \\
\hline
\end{tabular}


Table 5. Experimental and calculated total phase change enthalpy and entropy of database-Continued

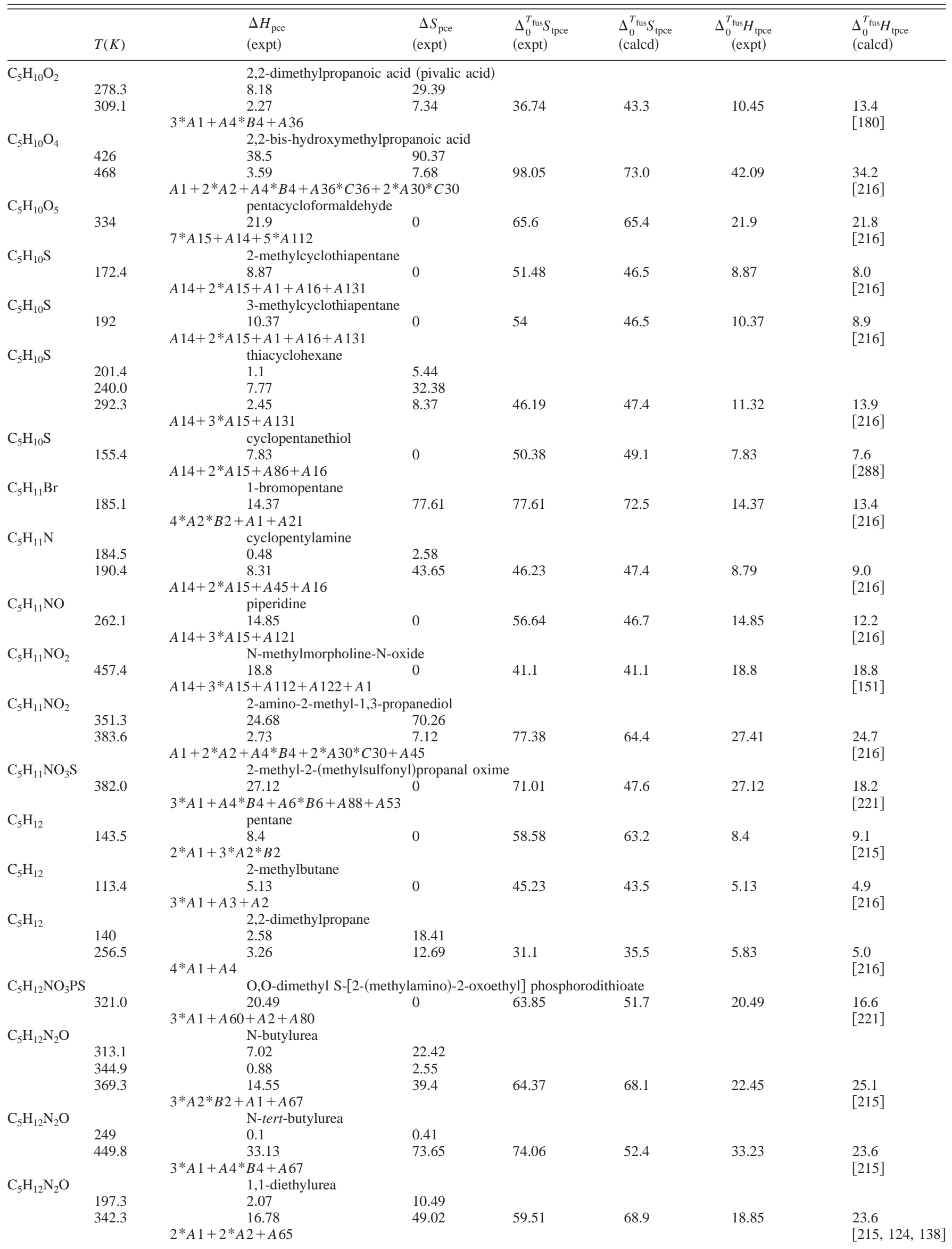


Table 5. Experimental and calculated total phase change enthalpy and entropy of database-Continued

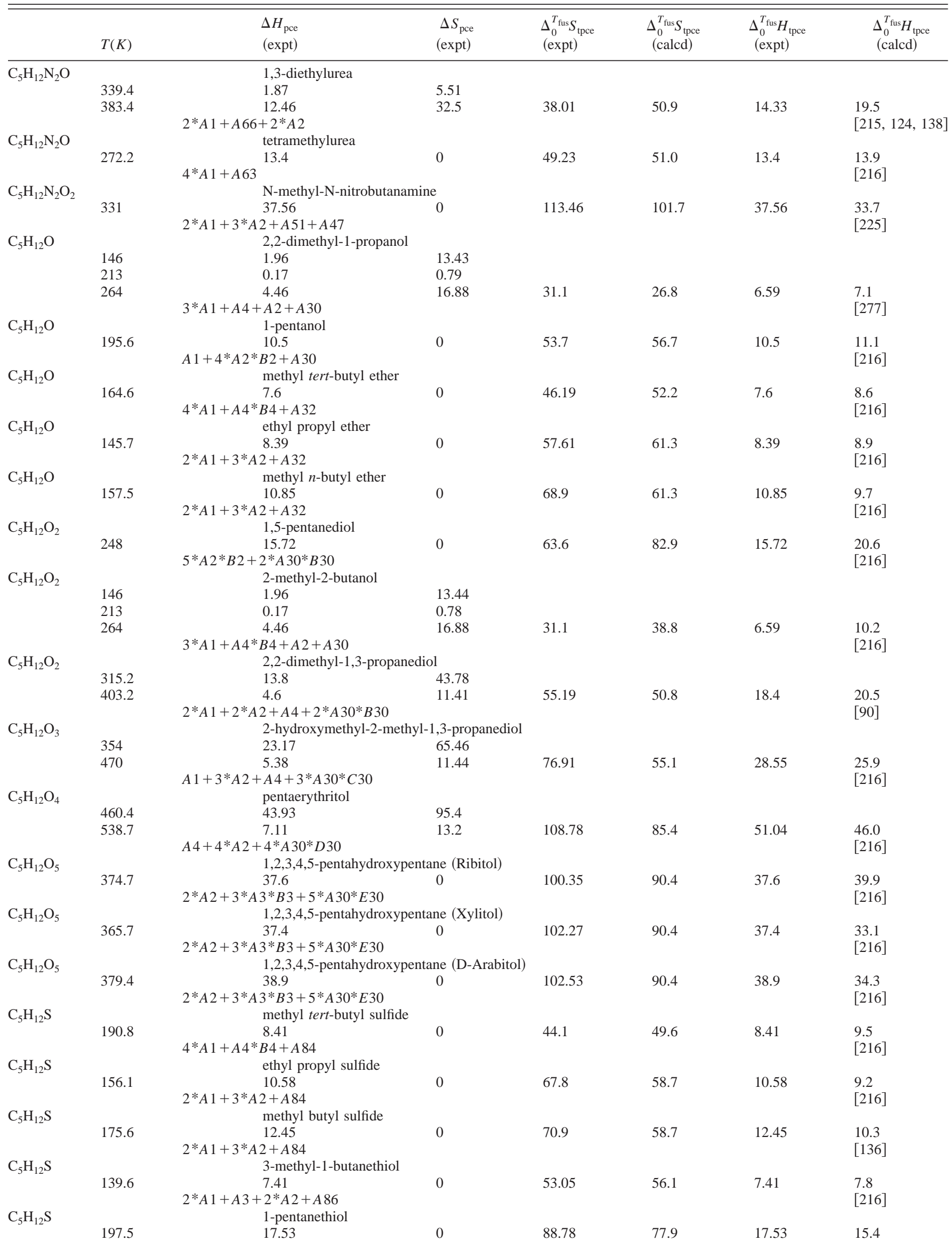


Table 5. Experimental and calculated total phase change enthalpy and entropy of database-Continued

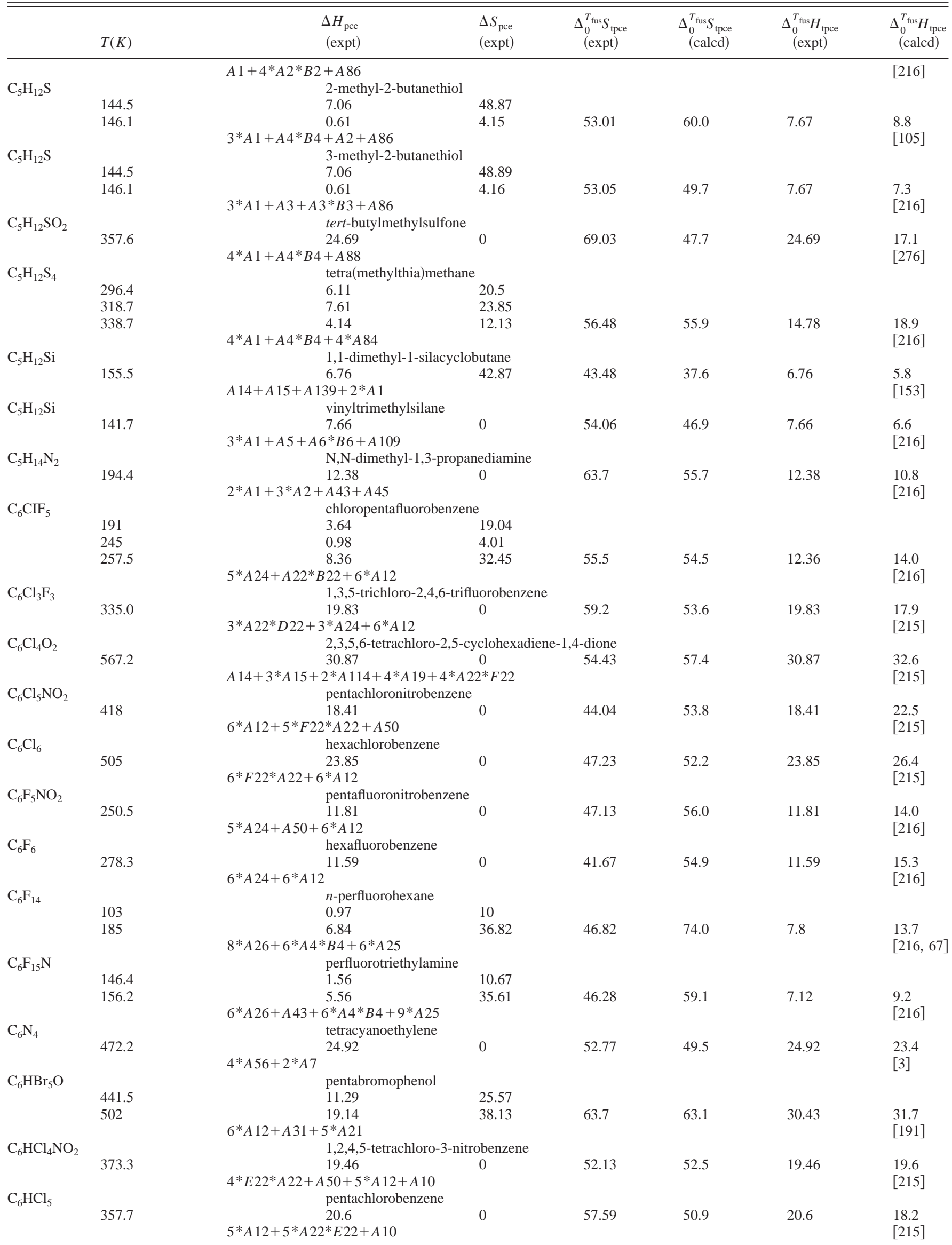


Table 5. Experimental and calculated total phase change enthalpy and entropy of database-Continued

\begin{tabular}{|c|c|c|c|c|c|c|c|}
\hline & $T(K)$ & $\begin{array}{l}\Delta H_{\mathrm{pce}} \\
(\text { expt) }\end{array}$ & $\begin{array}{l}\Delta S_{\text {pce }} \\
(\text { expt })\end{array}$ & $\begin{array}{l}\Delta_{0}^{T_{\text {fus }}} S_{\text {tpce }} \\
\quad(\operatorname{expt})\end{array}$ & $\begin{array}{l}\Delta_{0}^{T_{\text {fus }}} S_{\text {tpce }} \\
\quad(\text { calcd })\end{array}$ & $\begin{array}{l}\Delta_{0}^{T_{\text {fus }}} H_{\text {tpce }} \\
\quad(\operatorname{expt})\end{array}$ & $\begin{array}{l}\Delta_{0}^{T_{\text {fus }}} H_{\text {tpce }} \\
\text { (calcd) }\end{array}$ \\
\hline $\mathrm{C}_{6} \mathrm{HCl}_{5} \mathrm{O}$ & & pentachlorophenol & & & & & \\
\hline & 462.5 & $\begin{array}{c}17.15 \\
5 * A 22 * F 22+A 31+6 * A 12\end{array}$ & 0 & 37.08 & 56.3 & 17.15 & $\begin{array}{l}26.0 \\
{[215,191]}\end{array}$ \\
\hline $\mathrm{C}_{6} \mathrm{HF}_{5}$ & 225.7 & $\begin{array}{r}\text { pentafluorobenzene } \\
10.88 \\
5 * A 24+5 * A 12+A 10\end{array}$ & 0 & 48.24 & 53.2 & 10.88 & $\begin{array}{l}12.0 \\
{[215]}\end{array}$ \\
\hline $\mathrm{C}_{6} \mathrm{HF}_{5} \mathrm{O}$ & $\begin{array}{l}287 \\
310.6\end{array}$ & $\begin{aligned} \text { pentafluorophenol } \\
1.16 \\
16.41 \\
6 * A 12+A 31+5 * A 24\end{aligned}$ & $\begin{array}{l}4.04 \\
52.83\end{array}$ & 56.87 & 58.5 & 17.57 & $\begin{array}{l}18.2 \\
{[72]}\end{array}$ \\
\hline $\mathrm{C}_{6} \mathrm{H}_{2} \mathrm{Br}_{4}$ & $\begin{array}{l}306.8 \\
453.1\end{array}$ & $\begin{aligned} & 1,2,4,5 \text {-tetrabromobenzene } \\
& 0.34 \\
& 27.88 \\
& 4 * A 21+4 * A 12+2 * A 10\end{aligned}$ & $\begin{array}{l}1.09 \\
61.53\end{array}$ & 62.62 & 55.1 & 28.22 & $\begin{array}{l}25.0 \\
{[216]}\end{array}$ \\
\hline $\mathrm{C}_{6} \mathrm{H}_{2} \mathrm{Cl}_{4}$ & 320 & $\begin{array}{l}\text { 1,2,3,4-tetrachlorobenzene } \\
17 \\
4 * A 12+2 * A 10+4 * A 22 * D 22\end{array}$ & 0 & 53.13 & 49.6 & 17 & $\begin{array}{l}15.9 \\
{[215]}\end{array}$ \\
\hline $\mathrm{C}_{6} \mathrm{H}_{2} \mathrm{Cl}_{4}$ & 421.2 & $\begin{aligned} & 1,2,4,5 \text {-tetrachlorobenzene } \\
& 24.1 \\
& 4 * A 12+2 * A 10+4 * A 22 * D 22\end{aligned}$ & 0 & 57.22 & 49.6 & 24.1 & $\begin{array}{l}20.9 \\
{[215]}\end{array}$ \\
\hline $\mathrm{C}_{6} \mathrm{H}_{2} \mathrm{Cl}_{4}$ & 323.9 & $\begin{aligned} 1,2,3,5 \text {-tetrachlorobenzene } \\
19 \\
4 * A 12+2 * A 10+4 * A 22 * D 22 \\
\text { pentachloroaniline }\end{aligned}$ & 0 & 58.66 & 49.6 & 19 & $\begin{array}{l}16.1 \\
{[215]}\end{array}$ \\
\hline & 505.8 & $\begin{array}{c}18.7 \\
6 * A 12+5^{*} A 22^{*} F 22+A 45\end{array}$ & 0 & 36.97 & 57.4 & 18.7 & $\begin{array}{l}29.0 \\
{[215]}\end{array}$ \\
\hline $\mathrm{C}_{6} \mathrm{H}_{2} \mathrm{~F}_{4}$ & 233.3 & $\begin{array}{l}\text { 1,2,3,4-tetrafluorobenzene } \\
10.93 \\
4 * A 12+2 * A 10+4 * A 24\end{array}$ & 0 & 46.85 & 51.4 & 10.93 & $\begin{array}{l}12.0 \\
{[65]}\end{array}$ \\
\hline $\mathrm{C}_{6} \mathrm{H}_{2} \mathrm{~F}_{4}$ & 226.9 & $\begin{aligned} 1,2,3,5 \text {-tetrafluorobenzene } \\
10.67 \\
4 * A 12+2 * A 10+4 * A 24\end{aligned}$ & 0 & 47.01 & 51.4 & 10.67 & $\begin{array}{l}11.7 \\
{[65]}\end{array}$ \\
\hline $\mathrm{C}_{6} \mathrm{H}_{2} \mathrm{~F}_{4}$ & 277 & $\begin{aligned} 1,2,4,5 \text {-tetrafluorobenzene } \\
15.05 \\
4 * A 12+2 * A 10+4 * A 24 \\
\text { pentafluoroaniline }\end{aligned}$ & 0 & 54.31 & 51.4 & 15.05 & $\begin{array}{l}14.3 \\
{[65]}\end{array}$ \\
\hline & $\begin{array}{l}287.4 \\
306.8\end{array}$ & $\begin{array}{c}3.94 \\
14.27 \\
5 * A 24+A 45+6 * A 12\end{array}$ & $\begin{array}{l}13.71 \\
46.51\end{array}$ & 60.22 & 59.6 & 18.21 & $\begin{array}{l}18.3 \\
{[216]}\end{array}$ \\
\hline $\mathrm{C}_{6} \mathrm{H}_{3} \mathrm{BrCl}_{2} \mathrm{O}$ & 343.4 & $\begin{array}{l}\text { 4-bromo-2,5-dichlorophenol } \\
22.11 \\
2 * A 10+4 * A 12+2 * A 22 * D 22+A 21+A 31\end{array}$ & 0 & 64.39 & 55.0 & 22.11 & $\begin{array}{l}18.9 \\
{[221]}\end{array}$ \\
\hline $\mathrm{C}_{6} \mathrm{H}_{3} \mathrm{Br}_{3} \mathrm{O}$ & 366.2 & $\begin{array}{l}\text { 2,4,6-tribromophenol } \\
18.52 \\
4 * A 12+2 * A 10+3 * A 21+A 31\end{array}$ & 0 & 50.57 & 57.8 & 18.52 & $\begin{array}{l}21.2 \\
{[215]}\end{array}$ \\
\hline $\mathrm{C}_{6} \mathrm{H}_{3} \mathrm{Cl}_{3}$ & 326.9 & $\begin{array}{c}\text { 1,2,3-trichlorobenzene } \\
20.5 \\
3 * A 10+3 * A 12+3 * A 22 * C 22\end{array}$ & 0 & 62.71 & 48.4 & 20.5 & $\begin{array}{l}15.8 \\
{[215]}\end{array}$ \\
\hline $\mathrm{C}_{6} \mathrm{H}_{3} \mathrm{Cl}_{3}$ & 336.7 & $\begin{array}{r}1,3,5 \text {-trichlorobenzene } \\
18.2 \\
3 * A 10+3 * A 12+3 * A 22 * C 22\end{array}$ & 0 & 54.05 & 48.4 & 18.2 & $\begin{array}{l}16.3 \\
{[215]}\end{array}$ \\
\hline $\mathrm{C}_{6} \mathrm{H}_{3} \mathrm{Cl}_{3} \mathrm{O}$ & 340.3 & $\begin{aligned} & 2,4,5 \text {-trichlorophenol } \\
& 21.59 \\
& 4 * A 12+2 * A 10+3 * A 22 * D 22+A 31\end{aligned}$ & 0 & 63.44 & 53.7 & 21.59 & $\begin{array}{l}18.3 \\
{[221]}\end{array}$ \\
\hline $\mathrm{C}_{6} \mathrm{H}_{3} \mathrm{Cl}_{4} \mathrm{~N}$ & 337.2 & $\begin{array}{l}\text { 2-chloro-6-(trichloromethyl)py } \\
20.3 \\
3 * A 10+A 41+A 11+A 12+A 4 * B 4+4 * A 22 \\
1,3,5 \text {-trinitrobenzene }\end{array}$ & $\begin{array}{l}\text { ridine } \\
0 \\
2 * E 22\end{array}$ & 60.2 & 58.0 & 20.3 & $\begin{array}{l}19.6 \\
{[216]}\end{array}$ \\
\hline $6-6+3+3 \sigma_{6}$ & $\begin{array}{l}370 \\
380.3\end{array}$ & $\begin{array}{c}1.9 \\
14.8 \\
3 * A 10+3 * A 12+3 * A 50 * C 50\end{array}$ & $\begin{array}{l}5.13 \\
38.95\end{array}$ & 44.08 & 53.0 & 16.71 & $\begin{array}{l}20.2 \\
{[216]}\end{array}$ \\
\hline $\mathrm{C}_{6} \mathrm{H}_{3} \mathrm{~N}_{3} \mathrm{O}_{7}$ & 394.1 & $\begin{array}{c}\text { picric acid } \\
17.1 \\
2 * A 10+4 * A 12+3 * A 50+A 31\end{array}$ & 0 & 43.39 & 58.4 & 17.1 & $\begin{array}{l}23.0 \\
{[216]}\end{array}$ \\
\hline $\mathrm{C}_{6} \mathrm{H}_{3} \mathrm{~N}_{3} \mathrm{O}_{8}$ & 454.8 & $\begin{array}{c}2,4,6 \text {-trinitroresorcinol } \\
33.5 \\
A 10+5 * A 12+2 * A 31+3 * A 50\end{array}$ & 0 & 73.66 & 63.7 & 33.5 & $\begin{array}{l}29.0 \\
{[216]}\end{array}$ \\
\hline $\mathrm{C}_{6} \mathrm{H}_{4} \mathrm{BrCl}$ & & 1,2-bromochlorobenzene & & & & & \\
\hline
\end{tabular}


Table 5. Experimental and calculated total phase change enthalpy and entropy of database-Continued

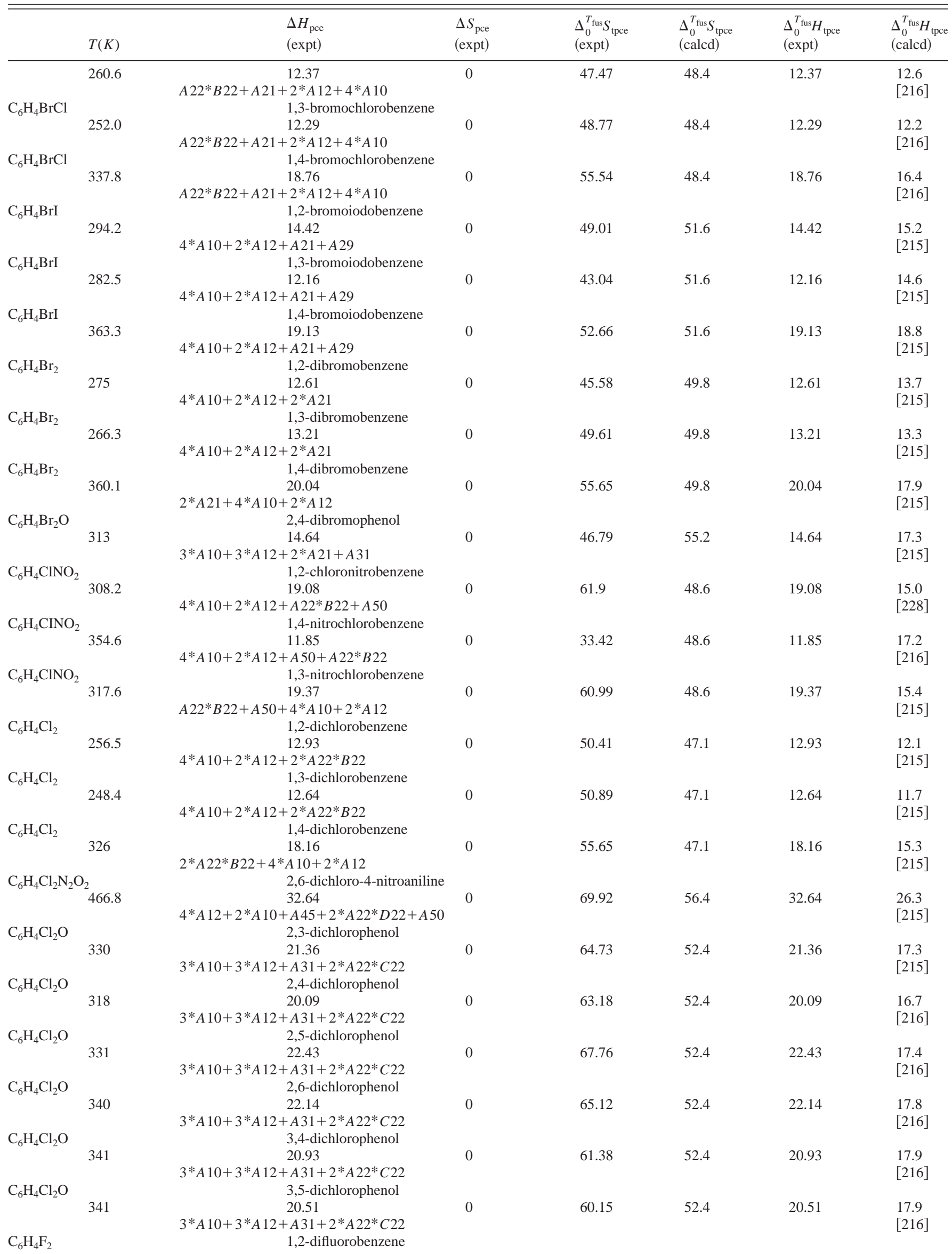


Table 5. Experimental and calculated total phase change enthalpy and entropy of database-Continued

\begin{tabular}{|c|c|c|c|c|c|c|c|}
\hline & $T(K)$ & $\begin{array}{l}\Delta H_{\mathrm{pce}} \\
(\operatorname{expt})\end{array}$ & $\begin{array}{l}\Delta S_{\text {pce }} \\
(\operatorname{expt})\end{array}$ & $\begin{array}{l}\Delta_{0}^{T_{\text {fus }}} S_{\text {tpce }} \\
\quad(\operatorname{expt})\end{array}$ & $\begin{array}{l}\Delta_{0}^{T_{\text {fus }}} S_{\text {tpce }} \\
\quad(\text { calcd })\end{array}$ & $\begin{array}{l}\Delta_{0}^{T_{\text {fus }}} H_{\text {tpce }} \\
\quad(\text { expt })\end{array}$ & $\begin{array}{l}\Delta_{0}^{T_{\text {fus }}} H_{\text {tpce }} \\
\quad \text { (calcd) }\end{array}$ \\
\hline & 226 & $\begin{array}{c}11.05 \\
4 * A 10+2 * A 12+2 * A 24\end{array}$ & 0 & 48.95 & 48.0 & 11.05 & $\begin{array}{l}10.8 \\
{[216]}\end{array}$ \\
\hline $\mathrm{C}_{6} \mathrm{H}_{4} \mathrm{~F}_{2}$ & & 1,3-difluorobenzene & & & & & \\
\hline & 186.8 & 0.83 & 4.43 & & & & \\
\hline & 204.0 & $\begin{array}{c}8.58 \\
2 * A 24+2 * A 12+4 * A 10\end{array}$ & 42.05 & 46.48 & 48.0 & 9.4 & $\begin{array}{l}9.8 \\
{[216]}\end{array}$ \\
\hline $\mathrm{C}_{6} \mathrm{H}_{4} \mathrm{I}_{2}$ & 296.6 & $\begin{array}{c}\text { 1,2-diiodobenzene } \\
14.01 \\
2 * A 29+2 * A 12+4 * A 10\end{array}$ & 0 & 47.24 & 53.5 & 14.01 & $\begin{array}{l}15.9 \\
{[215]}\end{array}$ \\
\hline $\mathrm{C}_{6} \mathrm{H}_{4} \mathrm{I}_{2}$ & 307.4 & $\begin{aligned} & \text { 1,3-diiodobenzene } 15.93 \\
& 2 * A 29+2 * A 12+4 * A 10\end{aligned}$ & 0 & 51.82 & 53.5 & 15.93 & $\begin{array}{l}16.4 \\
{[215]}\end{array}$ \\
\hline $\mathrm{C}_{6} \mathrm{H}_{4} \mathrm{I}_{2}$ & 402 & $\begin{array}{r}\text { 1,4-diiodobenzene } \\
22.37 \\
2 * A 29+2 * A 12+4 * A 10\end{array}$ & 0 & 55.65 & 53.5 & 22.37 & $\begin{array}{l}21.5 \\
{[215]}\end{array}$ \\
\hline $\mathrm{C}_{6} \mathrm{H}_{4} \mathrm{~N}_{2} \mathrm{O}_{4}$ & 396.1 & $\begin{aligned} & \text { 1,2-dinitrobenzene } \\
& 22.84 \\
& 4 * A 10+2 * A 12+2 * A 50\end{aligned}$ & 0 & 57.66 & 50.2 & 22.84 & $\begin{array}{l}19.9 \\
{[216]}\end{array}$ \\
\hline $\mathrm{C}_{6} \mathrm{H}_{4} \mathrm{~N}_{2} \mathrm{O}_{4}$ & 363.2 & $\begin{aligned} & \text { 1,3-dinitrobenzene } \\
& 17.36 \\
& 4 * A 10+2 * A 12+2 * A 50\end{aligned}$ & 0 & 47.82 & 50.2 & 17.36 & $\begin{array}{l}18.2 \\
{[215]}\end{array}$ \\
\hline $\mathrm{C}_{6} \mathrm{H}_{4} \mathrm{~N}_{2} \mathrm{O}_{4}$ & 446.7 & $\begin{aligned} & \text { 1,4-dinitrobenzene } \\
& 28.12 \\
& 4 * A 10+2 * A 12+2 * A 50\end{aligned}$ & 0 & 62.93 & 50.2 & 28.12 & $\begin{array}{l}22.4 \\
{[215]}\end{array}$ \\
\hline $\mathrm{C}_{6} \mathrm{H}_{4} \mathrm{~N}_{2} \mathrm{O}_{5}$ & 417 & $\begin{array}{l}\text { 2,3-dinitrophenol } \\
26.24 \\
3 * A 10+3 * A 12+2 * A 50+A 31\end{array}$ & 0 & 62.93 & 55.5 & 26.24 & $\begin{array}{l}23.2 \\
{[216]}\end{array}$ \\
\hline $\mathrm{C}_{6} \mathrm{H}_{4} \mathrm{~N}_{2} \mathrm{O}_{5}$ & 388 & $\begin{array}{l}\text { 2,4-dinitrophenol } \\
24.17 \\
3 * A 10+3 * A 12+2 * A 50+A 31 \\
2,5 \text {-dinitrophenol }\end{array}$ & 0 & 62.29 & 55.5 & 24.17 & $\begin{array}{l}21.6 \\
{[216]}\end{array}$ \\
\hline & 381 & $\begin{array}{c}23.73 \\
3 * A 10+3 * A 12+2 * A 50+A 31\end{array}$ & 0 & 62.28 & 55.5 & 23.73 & $\begin{array}{l}21.2 \\
{[216]}\end{array}$ \\
\hline $\mathrm{C}_{6} \mathrm{H}_{4} \mathrm{~N}_{2} \mathrm{O}_{5}$ & 336 & $\begin{array}{rl}\text { 2,6-dinitrophenol } & \\
19.58 \\
3 * A 10+3 * A & 12+2 * A 50+A 31 \\
3,4-d i n i t r o p h e n o l\end{array}$ & 0 & 58.27 & 55.5 & 19.58 & $\begin{array}{l}18.7 \\
{[216]}\end{array}$ \\
\hline $\mathrm{C}_{6} \mathrm{H}_{4} \mathrm{O}_{2}$ & 407 & $\begin{array}{c}25.37 \\
3 * A 10+3 * A 12+2 * A 50+A 31 \\
p \text {-benzoquinone }\end{array}$ & 0 & 62.33 & 55.5 & 25.37 & $\begin{array}{l}22.6 \\
{[216]}\end{array}$ \\
\hline $\mathrm{C}_{6} \mathrm{H}_{5} \mathrm{Br}$ & 388 & $\begin{array}{c}18.45 \\
3 * A 15+A 14+4 * A 18 * B 18+2 * A 114 \\
\text { bromobenzene }\end{array}$ & 0 & 47.56 & 29.4 & 18.45 & $\begin{array}{l}11.4 \\
{[215]}\end{array}$ \\
\hline & 242.4 & $\begin{array}{c}10.7 \\
5 * A 10+A 12+A 21\end{array}$ & 0 & 44.2 & 47.1 & 10.7 & $\begin{array}{l}11.4 \\
{[216]}\end{array}$ \\
\hline $\mathrm{C}_{6} \mathrm{H}_{5} \mathrm{BrO}$ & 336 & $\begin{array}{r}\text { 4-bromophenol } \\
16.57 \\
A 21+4 * A 10+2 * A 12+A 31 \\
\text { chlorobenzene }\end{array}$ & 0 & 49.32 & 52.5 & 16.57 & $\begin{array}{l}17.6 \\
{[216]}\end{array}$ \\
\hline & 227.9 & $\begin{array}{r}9.55 \\
5 * A 10+A 22+A 12\end{array}$ & 0 & 41.92 & 40.4 & 9.55 & $\begin{array}{l}9.2 \\
{[216]}\end{array}$ \\
\hline $\mathrm{C}_{6} \mathrm{H}_{5} \mathrm{ClO}$ & 276 & $\begin{array}{l}\text { 2-chlorophenol } \\
0.09\end{array}$ & 0.33 & & & & \\
\hline $\mathrm{C}_{6} \mathrm{H}_{5} \mathrm{ClO}$ & 283 & $\begin{array}{c}12.52 \\
4 * A 10+2 * A 12+A 22 * B 22+A 31 \\
\text { 3-chlorophenol }\end{array}$ & 44.24 & 44.57 & 51.2 & 12.61 & $\begin{array}{l}14.5 \\
{[215]}\end{array}$ \\
\hline & 305.8 & $\begin{array}{c}14.91 \\
4 * A 10+2 * A 12+A 22 * B 22+A 31\end{array}$ & 0 & 48.76 & 51.2 & 14.91 & $\begin{array}{l}15.6 \\
{[215]}\end{array}$ \\
\hline $\mathrm{C}_{6} \mathrm{H}_{5} \mathrm{ClO}$ & 315.9 & $\begin{array}{c}\text { 4-chlorophenol } \\
14.07 \\
4 * A 10+2 * A 12+A 22 * B 22+A 31\end{array}$ & 0 & 44.54 & 51.2 & 14.07 & $\begin{array}{l}16.2 \\
{[215]}\end{array}$ \\
\hline $\mathrm{C}_{6} \mathrm{H}_{5} \mathrm{Cl}_{2} \mathrm{~N}$ & & 2,6-dichloro-4-benzenamine & & & & & \\
\hline $\mathrm{C}_{6} \mathrm{H}_{5} \mathrm{Cl}_{3} \mathrm{Si}$ & 467.2 & $\begin{array}{c}29.48 \\
3 * A 10+3 * A 12+2 * A 22 * C 22+A 45 \\
\text { phenyltrichlorosilane }\end{array}$ & 0 & 63.11 & 53.5 & 29.48 & $\begin{array}{l}25.0 \\
{[221]}\end{array}$ \\
\hline $\mathrm{C}_{6} \mathrm{H}_{5} \mathrm{~F}$ & 233.4 & $\begin{array}{c}11.66 \\
5 * A 10+A 11+3 * A 22 * D 22+A 109 \\
\text { fluorobenzene }\end{array}$ & 0 & 49.96 & 48.9 & 11.66 & $\begin{array}{l}11.4 \\
{[216]}\end{array}$ \\
\hline
\end{tabular}


Table 5. Experimental and calculated total phase change enthalpy and entropy of database-Continued

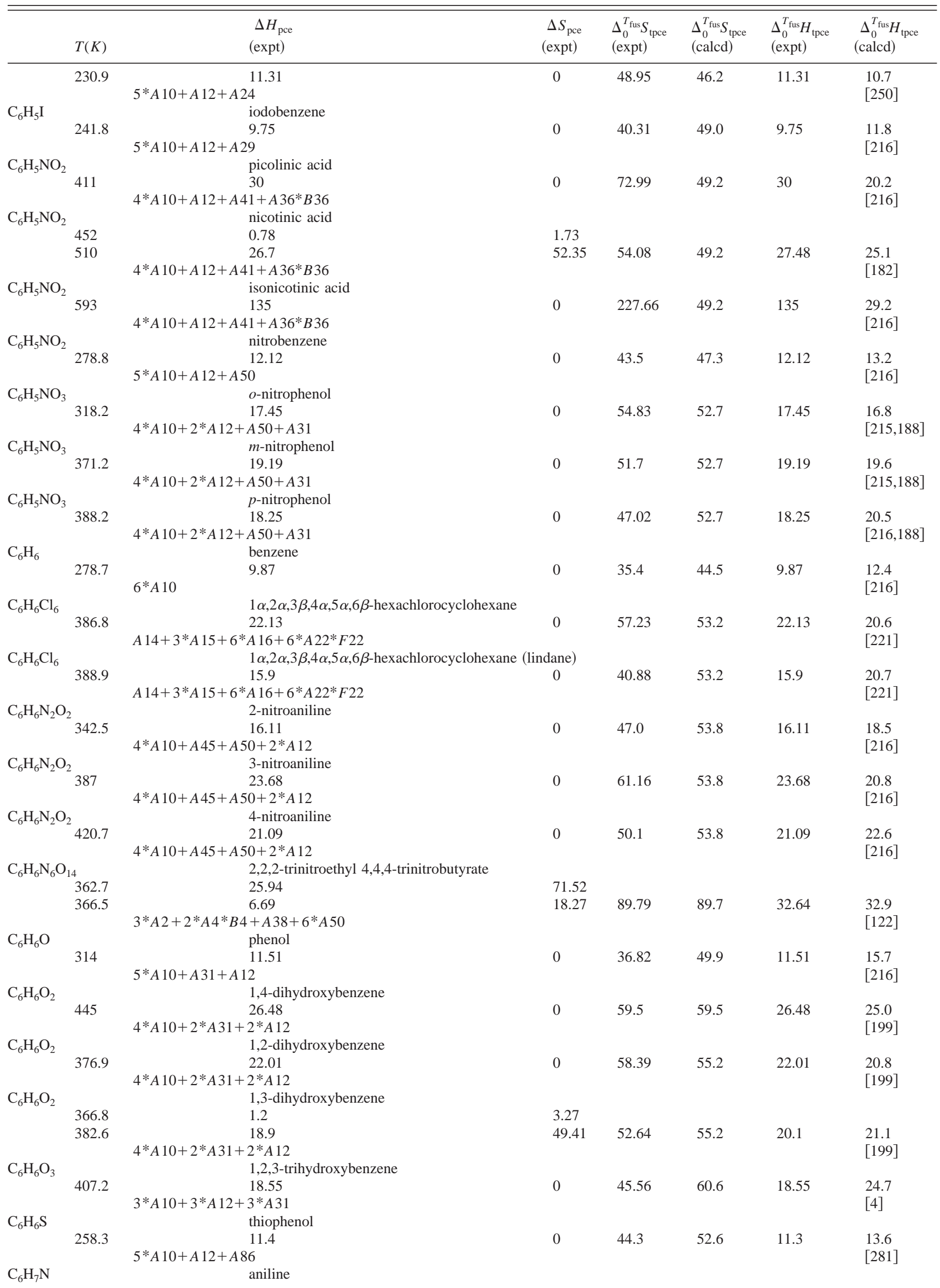


Table 5. Experimental and calculated total phase change enthalpy and entropy of database-Continued

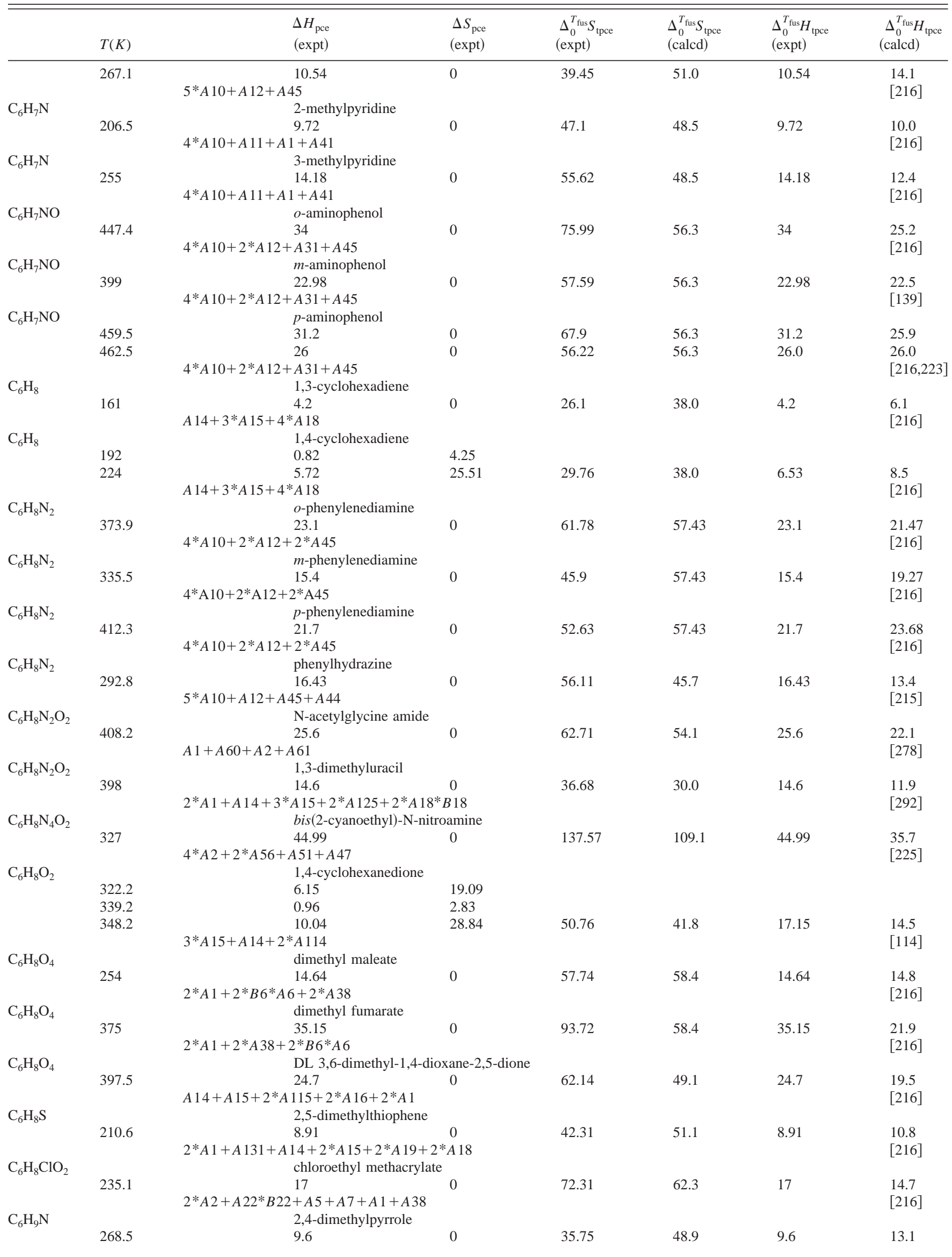


Table 5. Experimental and calculated total phase change enthalpy and entropy of database-Continued

\begin{tabular}{|c|c|c|c|c|c|c|c|}
\hline & $T(K)$ & $\begin{array}{l}\Delta H_{\mathrm{pce}} \\
(\operatorname{expt})\end{array}$ & $\begin{array}{l}\Delta S_{\mathrm{pce}} \\
(\mathrm{expt})\end{array}$ & $\underset{\text { (expt) }}{\Delta_{0}^{T_{\text {fus }}} S_{\text {tpce }}}$ & $\begin{array}{l}\Delta_{0}^{T_{\text {fus }}} S_{\text {tpce }} \\
\text { (calcd) }\end{array}$ & $\begin{array}{l}\Delta_{0}^{T_{\text {fus }}} H_{\text {tpce }} \\
(\text { expt })\end{array}$ & $\begin{array}{l}\Delta_{0}^{T_{\text {fus }}} H_{\text {tpce }} \\
\text { (calcd) }\end{array}$ \\
\hline \multicolumn{7}{|c|}{$A 14+2 * A 15+A 18+2 * A 1+A 121+A 18 * B 18+2 * A 19$} & {$[70]$} \\
\hline \multirow[t]{3}{*}{$\mathrm{C}_{6} \mathrm{H}_{9} \mathrm{~N}$} & & $2,5-\mathrm{dim}$ & & & & & \\
\hline & 280.9 & 9.3 & 0 & 33.09 & 50.4 & 9.3 & 14.2 \\
\hline & & $A 14+2 * A 15+2 * A 18$ & 19 & & & & {$[216]$} \\
\hline \multirow[t]{3}{*}{$\mathrm{C}_{6} \mathrm{H}_{9} \mathrm{NS}$} & & $2,4,5-\mathrm{tr}$ & & & & & \\
\hline & 240.7 & 9 & 0 & 37.39 & 60.2 & 9 & 14.5 \\
\hline & & $A 14+2 * A 15+3 * A 19$ & & & & & {$[61]$} \\
\hline \multirow[t]{4}{*}{$\mathrm{C}_{6} \mathrm{H}_{10}$} & & cyclohe & & & & & \\
\hline & 138.7 & 4.23 & 30.5 & & & & \\
\hline & 169.7 & 3.28 & 19.35 & 49.85 & 41.3 & 7.51 & 7.0 \\
\hline & & $A 14+3 * A 15+2 * A 18$ & & & & & {$[216]$} \\
\hline \multirow[t]{5}{*}{$\mathrm{C}_{6} \mathrm{H}_{10} \mathrm{~N}_{2} \mathrm{O}$} & & 2,3-dia & ne $\mathrm{N}-\mathrm{ox}$ & & & & \\
\hline & 359.3 & 5.02 & 13.97 & & & & \\
\hline & 399.3 & 8.05 & 20.16 & & & & \\
\hline & 438 & 3.84 & 8.77 & 42.9 & 47.6 & 16.91 & 20.8 \\
\hline & & $2 * A 14+2 * A 15+2 * A$ & & & & & {$[42]$} \\
\hline \multirow[t]{4}{*}{$\mathrm{C}_{6} \mathrm{H}_{10} \mathrm{O}$} & & cyclohe & & & & & \\
\hline & 220.8 & 8.66 & 39.22 & & & & \\
\hline & 245.2 & 1.33 & 5.42 & 44.64 & 43.1 & 9.99 & 10.6 \\
\hline & & $A 14+3 * A 15+A 114$ & & & & & {$[156]$} \\
\hline \multirow[t]{4}{*}{$\mathrm{C}_{6} \mathrm{H}_{10} \mathrm{O}$} & & cyclohe & & & & & \\
\hline & 193.1 & 9.54 & 49.38 & & & & \\
\hline & 238.1 & 1.06 & 4.47 & 53.85 & 42.2 & 10.6 & 10.1 \\
\hline & & $2 * A 14+A 15+A 112+$ & & & & & {$[156]$} \\
\hline \multirow[t]{3}{*}{$\mathrm{C}_{6} \mathrm{H}_{10} \mathrm{O}_{2}$} & & $\varepsilon$-capro & & & & & \\
\hline & 272 & 13.82 & 50.81 & 50.79 & 51.3 & 13.82 & 14.0 \\
\hline & & $A 14+4 * A 15+A 115$ & & & & & {$[32]$} \\
\hline \multirow[t]{4}{*}{$\mathrm{C}_{6} \mathrm{H}_{10} \mathrm{O}_{3}$} & & 2,2-dim & ponate & & & & \\
\hline & 324.1 & 10.3 & 31.78 & & & & \\
\hline & 387.2 & 5.62 & 14.52 & 46.31 & 46.3 & 15.92 & 17.9 \\
\hline & & $A 14+3 * A 15+A 116+$ & & & & & {$[200]$} \\
\hline \multirow[t]{3}{*}{$\mathrm{C}_{6} \mathrm{H}_{10} \mathrm{O}_{4}$} & & adipic & & & & & \\
\hline & 426.4 & 34.85 & 0 & 81.73 & 69.6 & 34.85 & 29.7 \\
\hline & & $4 * A 2 * B 2+2 * A 36 * B$ & & & & & {$[340]$} \\
\hline $\mathrm{C}_{6} \mathrm{H}_{10} \mathrm{O}_{6}$ & & $(d l) \operatorname{din}$ & & & & & \\
\hline & 360.2 & 26.94 & 0 & 74.81 & 76.7 & 26.94 & 27.6 \\
\hline & & $2 * A 38+2 * A 3 * B 3+2$ & & & & & {$[220]$} \\
\hline $\mathrm{C}_{6} \mathrm{H}_{10} \mathrm{O}_{6}$ & & (d) $\operatorname{dim}$ & & & & & \\
\hline & 322.2 & 17.36 & 0 & 53.89 & 76.7 & 17.36 & 24.7 \\
\hline & & $2 * A 38+2 * A 3 * B 3+2$ & & & & & {$[220]$} \\
\hline $\mathrm{C}_{6} \mathrm{H}_{11} \mathrm{Br}$ & & bromoc & & & & & \\
\hline & 216.9 & 10.79 & 0 & 49.75 & 47.3 & 10.79 & 10.3 \\
\hline & & $A 14+3 * A 15+A 21+$ & & & & & {$[190]$} \\
\hline $\mathrm{C}_{6} \mathrm{H}_{11} \mathrm{Cl}$ & & chloroc & & & & & \\
\hline & 120 & 0.05 & 0.42 & & & & \\
\hline & 220.4 & 8.01 & 36.35 & & & & \\
\hline & 229.3 & 2.04 & 8.91 & 45.67 & 40.5 & 10.1 & 9.3 \\
\hline & & $A 14+3 * A 15+A 16+$ & & & & & [229] \\
\hline $\mathrm{C}_{6} \mathrm{H}_{11} \mathrm{NO}$ & & cyclohe & & & & & \\
\hline & 240.8 & 0.01 & 0.06 & & & & \\
\hline & 362.6 & 12.7 & 35.02 & & & & \\
\hline & 273.4 & 0.09 & 0.34 & 35.43 & 45.8 & 12.81 & 12.5 \\
\hline & & $A 14+3 * A 15+A 19+$ & & & & & {$[5]$} \\
\hline $\mathrm{C}_{6} \mathrm{H}_{11} \mathrm{NO}$ & & $e$-capro & & & & & \\
\hline & 343.3 & 16.1 & 0 & 46.89 & 50.9 & 16.1 & 17.5 \\
\hline & & $A 14+4 * A 15+A 124$ & & & & & {$[6]$} \\
\hline $\mathrm{C}_{6} \mathrm{H}_{11} \mathrm{NO}_{3}$ & & $\mathrm{~N}$-dime & acid & & & & \\
\hline & 431.4 & 36.97 & 0 & 85.71 & 54.4 & 36.97 & 23.5 \\
\hline & & $2 * A 1+2 * A 2+A 36^{*} L$ & & & & & [221] \\
\hline $\mathrm{C}_{6} \mathrm{H}_{11} \mathrm{~N}_{2} \mathrm{O}_{3}$ & & S-2,3-d & xо- $1,3,4$ & 1-3-ylmethyl & & & \\
\hline & & $\mathrm{O}, \mathrm{O}-\mathrm{di}$ & oate & & & & \\
\hline & 315.1 & 28.54 & 0 & 90.59 & 90.7 & 28.54 & 28.6 \\
\hline & & $3 * \mathrm{~A} 1+\mathrm{A} 14+2 * \mathrm{~A} 15+$ & $A 32+A$ & & & & {$[221]$} \\
\hline $\mathrm{C}_{6} \mathrm{H}_{12}$ & & cyclohe & & & & & \\
\hline & 186.1 & 6.74 & 36.2 & & & & \\
\hline & 279.8 & 2.68 & 9.57 & 45.77 & 44.5 & 9.41 & 12.5 \\
\hline & & $A 14+3 * A 15$ & & & & & {$[216]$} \\
\hline $\mathrm{C}_{6} \mathrm{H}_{12}$ & & methyl & & & & & \\
\hline & 130.7 & 6.93 & 0 & 53.01 & 43.6 & 6.93 & 5.7 \\
\hline & & $A 14+A 16+A 1+2 * A$ & & & & & {$[216]$} \\
\hline
\end{tabular}


Table 5. Experimental and calculated total phase change enthalpy and entropy of database-Continued

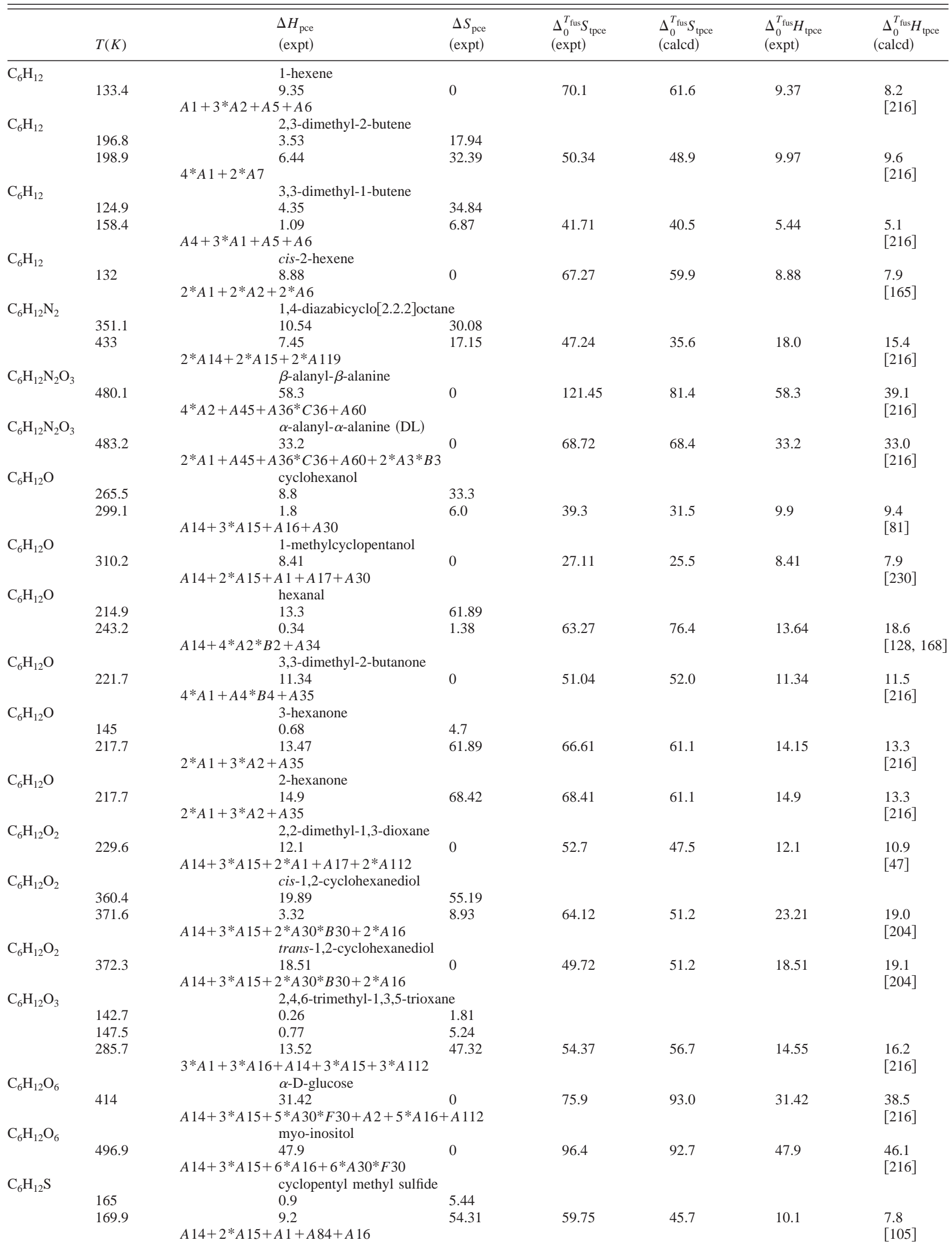


PHASE CHANGE ENTHALPIES AND ENTROPIES

Table 5. Experimental and calculated total phase change enthalpy and entropy of database-Continued

\begin{tabular}{|c|c|c|c|c|c|c|c|}
\hline & $T(K)$ & $\begin{array}{l}\Delta H_{\mathrm{pce}} \\
(\mathrm{expt})\end{array}$ & $\begin{array}{l}\Delta S_{\mathrm{pce}} \\
(\mathrm{expt})\end{array}$ & $\begin{array}{l}\Delta_{0}^{T_{\text {fus }}} S_{\text {tpce }} \\
(\text { expt) }\end{array}$ & $\begin{array}{l}\Delta_{0}^{T_{\text {fus }}} S_{\text {tpce }} \\
\text { (calcd) }\end{array}$ & $\begin{array}{l}\Delta_{0}^{T_{\text {fus }}} H_{\text {tpce }} \\
(\operatorname{expt})\end{array}$ & $\begin{array}{l}\Delta_{0}^{T_{\text {fus }}} H_{\text {tpce }} \\
\text { (calcd) }\end{array}$ \\
\hline \multirow[t]{3}{*}{$\mathrm{C}_{6} \mathrm{H}_{12} \mathrm{~S}$} & & cyclohexanethiol & & & & & \\
\hline & 189.6 & 10 & 0 & 52.72 & 52.8 & 10.0 & 10.0 \\
\hline & & $A 14+3 * A 15+A 86+A 16$ & & & & & {$[341]$} \\
\hline \multirow[t]{3}{*}{$\mathrm{C}_{6} \mathrm{H}_{13} \mathrm{Br}$} & & 1-bromohexane & & & & & \\
\hline & 188.1 & 18.05 & 0 & 95.98 & 81.8 & 18.05 & 15.4 \\
\hline & & $A 1+5 * A 2 * B 2+A 21$ & & & & & {$[216]$} \\
\hline \multirow[t]{3}{*}{$\mathrm{C}_{6} \mathrm{H}_{13} \mathrm{~N}$} & & 2-methylpiperidine & & & & & \\
\hline & 269.4 & 18.58 & 0 & 68.99 & 49.5 & 18.58 & 13.3 \\
\hline & & $A 14+3 * A 15+A 121+A 1+A 16$ & & & & & {$[216]$} \\
\hline \multirow[t]{3}{*}{$\mathrm{C}_{6} \mathrm{H}_{13} \mathrm{NO}$} & & hexanamide & & & & & \\
\hline & 374 & 25.1 & 0 & 67.12 & 82.8 & 25.1 & 31.0 \\
\hline & & $4 * A 2 * B 2+A 1+A 61$ & & & & & {$[279]$} \\
\hline \multirow[t]{3}{*}{$\mathrm{C}_{6} \mathrm{H}_{14}$} & & $n$-hexane & & & & & \\
\hline & 177.8 & 13.08 & 0 & 73.22 & 72.5 & 13.08 & 12.9 \\
\hline & & $2 * A 1+4 * A 2 * B 2$ & & & & & {$[216]$} \\
\hline \multirow[t]{5}{*}{$\mathrm{C}_{6} \mathrm{H}_{14}$} & & 2,3-dimethylbutane & & & & & \\
\hline & 136.1 & 6.43 & 47.22 & & & & \\
\hline & 107 & 2.37 & 22.13 & & & & \\
\hline & 145.2 & 0.79 & 5.47 & 52.96 & 37.6 & 9.59 & 5.1 \\
\hline & & $4 * A 1+2 * A 3$ & & & & & {$[216]$} \\
\hline \multirow[t]{3}{*}{$\mathrm{C}_{6} \mathrm{H}_{14}$} & & 3-methylpentane & & & & & \\
\hline & 110.3 & 5.31 & 0 & 48.17 & 50.6 & 5.31 & 5.6 \\
\hline & & $2 * A 2+3 * A 1+A 3$ & & & & & {$[216]$} \\
\hline \multirow{3}{*}{$\mathrm{C}_{6} \mathrm{H}_{14}$} & & 2-methylpentane & & & & & \\
\hline & 119.6 & 6.27 & 0 & 52.43 & 50.6 & 6.27 & 6.05 \\
\hline & & $2 * A 2+3 * A 1+A 3$ & & & & & {$[216]$} \\
\hline \multirow[t]{5}{*}{$\mathrm{C}_{6} \mathrm{H}_{14}$} & & 2,2-dimethylbutane & & & & & \\
\hline & 126.8 & 5.4 & 42.57 & & & & \\
\hline & 140.8 & 0.28 & 2.02 & & & & \\
\hline & 174.3 & 0.58 & 3.31 & 45.88 & 42.6 & 6.26 & 7.4 \\
\hline & & $4 * A 1+A 2+A 4$ & & & & & {$[216]$} \\
\hline \multirow[t]{3}{*}{$\mathrm{C}_{6} \mathrm{H}_{14} \mathrm{O}$} & & 1-hexanol & & & & & \\
\hline & 225.8 & 15.48 & 0 & 68.56 & 66.0 & 15.48 & 14.9 \\
\hline & & $A 1+5^{*} A 2 * B 2+A 30$ & & & & & {$[216]$} \\
\hline \multirow[t]{3}{*}{$\mathrm{C}_{6} \mathrm{H}_{14} \mathrm{O}$} & & isopropyl ether & & & & & \\
\hline & 187.8 & 12.05 & 0 & 64.02 & 55.7 & 12.05 & 10.5 \\
\hline & & $4 * A 1+2 * A 3 * B 3+A 32$ & & & & & {$[66]$} \\
\hline $\mathrm{C}_{6} \mathrm{H}_{14} \mathrm{O}$ & & 4-oxaheptane & & & & & \\
\hline & 158.4 & 10.77 & 0 & 67.99 & 68.4 & 10.77 & 10.8 \\
\hline & & $2 * A 1+4 * A 2+A 32$ & & & & & {$[216]$} \\
\hline $\mathrm{C}_{6} \mathrm{H}_{14} \mathrm{O}_{2}$ & & 2,3-dimethyl-2,3-but & & & & & \\
\hline & 316.2 & 14.7 & 0 & 46.49 & 60.8 & 14.7 & 19.2 \\
\hline & & $4 * A 1+2 * A 4 * B 4+2 * B 30 * A 30$ & & & & & {$[231]$} \\
\hline $\mathrm{C}_{6} \mathrm{H}_{14} \mathrm{O}_{2}$ & & 1,6-hexanediol & & & & & \\
\hline & 320.6 & 25.52 & 0 & 79.6 & 92.2 & 25.52 & 29.0 \\
\hline & & $2 * A 30 * B 30+6 * A 2 * B 2$ & & & & & {$[216]$} \\
\hline $\mathrm{C}_{6} \mathrm{H}_{14} \mathrm{O}_{3}$ & & 2,5,8-trioxanonane & & & & & \\
\hline & 209.1 & 17.8 & 0 & 85.1 & 77.8 & 17.8 & 16.3 \\
\hline & & $2 * A 1+4 * A 2+3 * A 32$ & & & & & {$[216]$} \\
\hline $\mathrm{C}_{6} \mathrm{H}_{14} \mathrm{O}_{6}$ & & D sorbitol & & & & & \\
\hline & 366.5 & 30.2 & 0 & 82.4 & 111.7 & 30.2 & 40.9 \\
\hline & & $2 * A 2+4 * A 3 * B 3+6 * A 30 * F 30$ & & & & & \\
\hline & & & & & & & {$[216]$} \\
\hline $\mathrm{C}_{6} \mathrm{H}_{14} \mathrm{O}_{6}$ & & dulcitol & & & & & \\
\hline & 460.3 & 65.1 & 0 & 141.4 & 111.7 & 65.1 & 51.4 \\
\hline & & $2 * A 2+4 * A 3 * B 3+6 * A 30 * F 30$ & & & & & \\
\hline & & & & & & & {$[216]$} \\
\hline $\mathrm{C}_{6} \mathrm{H}_{14} \mathrm{O}_{6}$ & & D mannitol & & & & & \\
\hline & 439.1 & 56.1 & 0 & 127.8 & 111.7 & 56.1 & 49.0 \\
\hline & 438.7 & 50.6 & 0 & 115.3 & 111.7 & 50.6 & 49.0 \\
\hline & & $2 * A 2+4^{*} A 3 * B 3+6^{*} A 30^{*} F 30$ & & & & & {$[216,394]$} \\
\hline $\mathrm{C}_{6} \mathrm{H}_{14} \mathrm{~S}$ & & diisopropyl sulfide & & & & & \\
\hline & 195.1 & 10.42 & 0 & 53.39 & 52.8 & 10.42 & 10.4 \\
\hline & & $4 * A 1+2 * A 3 * B 3+A 84$ & & & & & {$[341]$} \\
\hline $\mathrm{C}_{6} \mathrm{H}_{14} \mathrm{~S}$ & & dipropyl sulfide & & & & & \\
\hline & 170.4 & 12.14 & 0 & 71.25 & 65.8 & 12.14 & 11.2 \\
\hline & & $2 * A 1+4 * A 2+A 84$ & & & & & {$[216]$} \\
\hline
\end{tabular}


Table 5. Experimental and calculated total phase change enthalpy and entropy of database-Continued

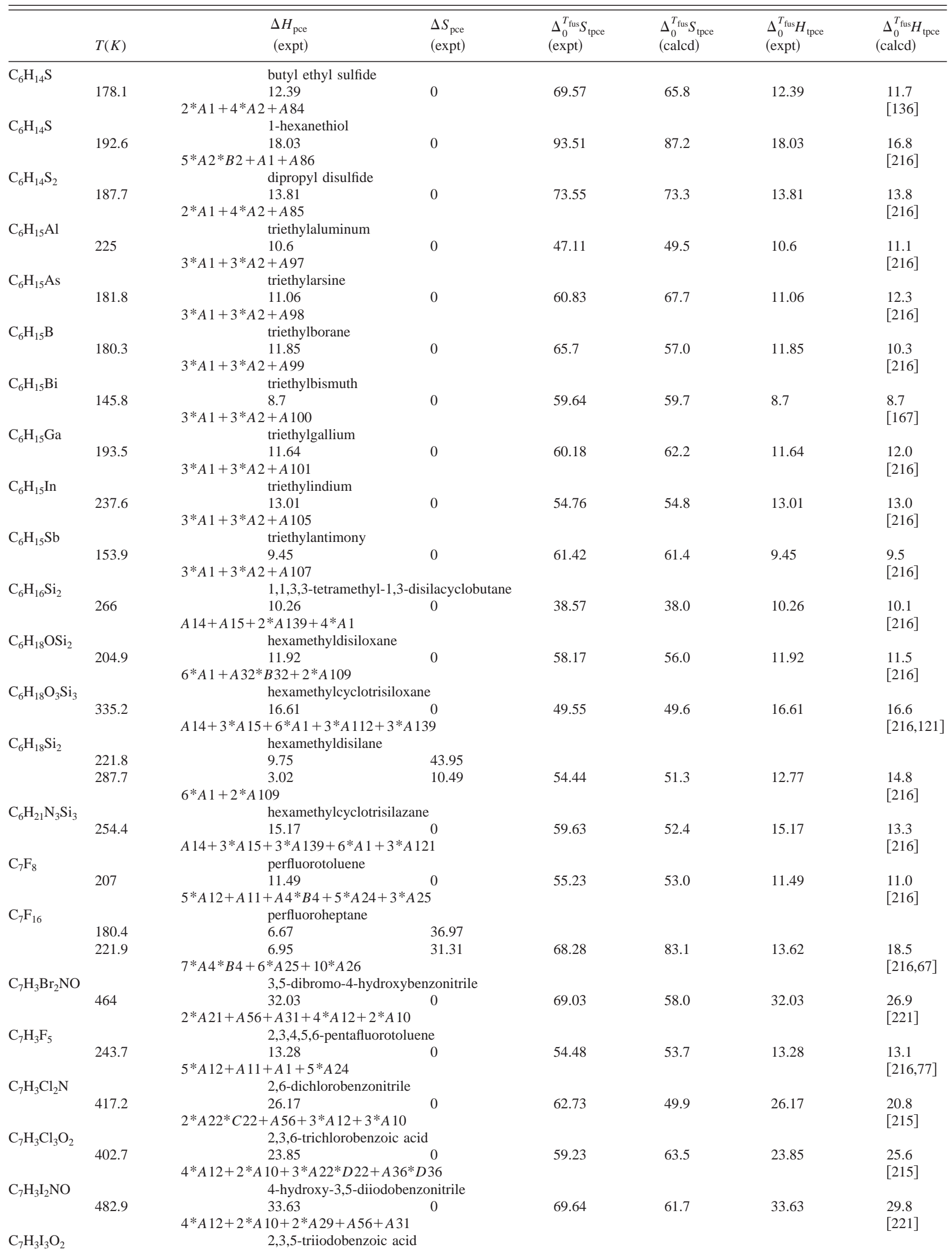


Table 5. Experimental and calculated total phase change enthalpy and entropy of database-Continued

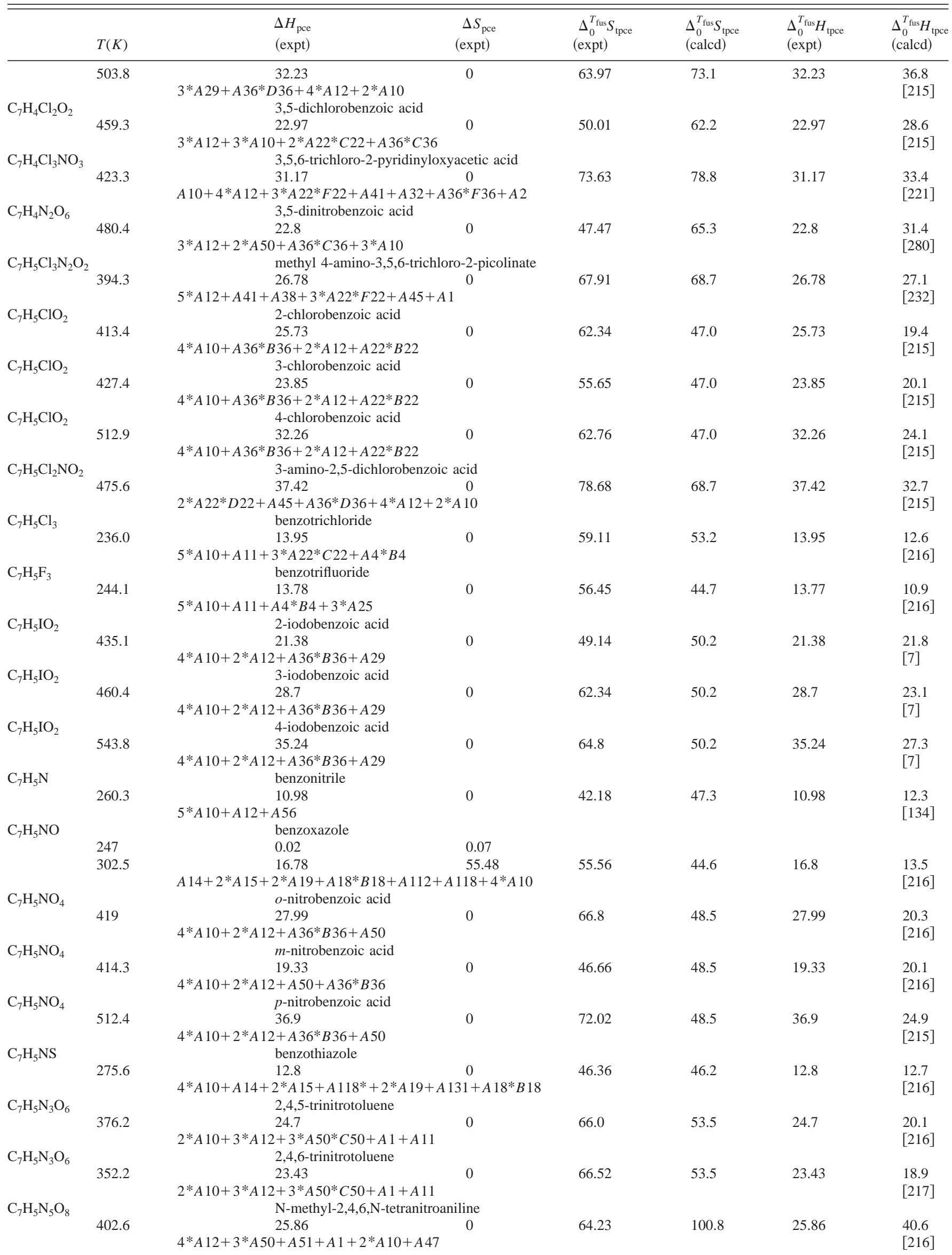


Table 5. Experimental and calculated total phase change enthalpy and entropy of database-Continued

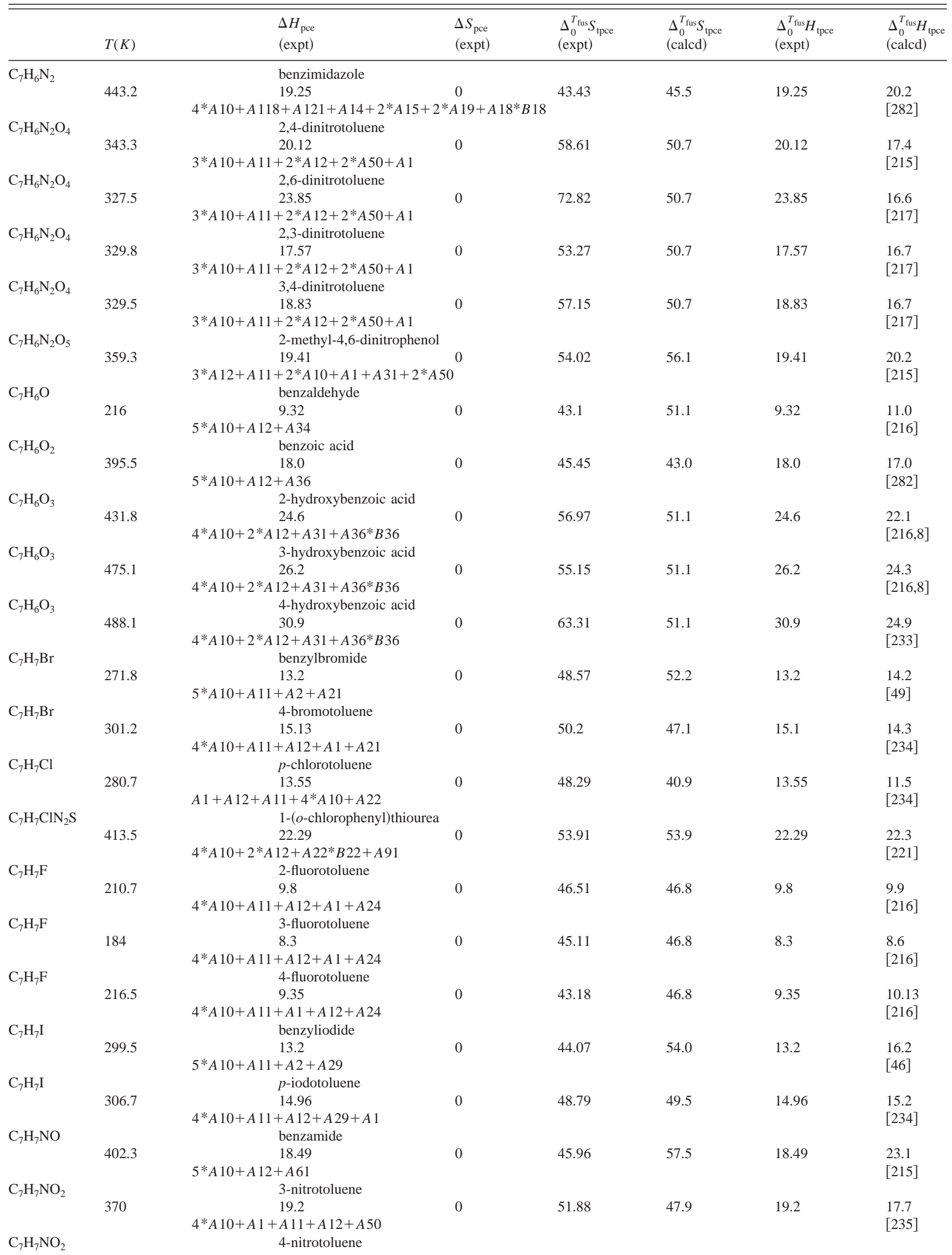


Table 5. Experimental and calculated total phase change enthalpy and entropy of database-Continued

\begin{tabular}{|c|c|c|c|c|c|c|c|}
\hline & $T(K)$ & $\begin{array}{l}\Delta H_{\mathrm{pce}} \\
(\text { expt) }\end{array}$ & $\begin{array}{l}\Delta S_{\text {pce }} \\
(\text { expt })\end{array}$ & $\begin{array}{l}\Delta_{0}^{T_{\text {fus }}} S_{\text {tpce }} \\
(\operatorname{expt})\end{array}$ & $\begin{array}{l}\Delta_{0}^{T_{\text {fus }}} S_{\text {tpce }} \\
\text { (calcd) }\end{array}$ & $\begin{array}{l}\Delta_{0}^{T_{\text {fus }}} H_{\text {tpce }} \\
(\text { expt) }\end{array}$ & $\begin{array}{l}\Delta_{0}^{T_{\text {fus }}} H_{\text {tpce }} \\
\text { (calcd) }\end{array}$ \\
\hline & 318 & 16.9 & 53.1 & & & & \\
\hline & 324.8 & 16.81 & 0 & 51.76 & 47.9 & 16.81 & 15.6 \\
\hline & & $4 * A 10+A 12+A 11+A 1+A 50$ & & & & & {$[215]$} \\
\hline \multirow{3}{*}{$\mathrm{C}_{7} \mathrm{H}_{7} \mathrm{NO}_{2}$} & & 2-aminobenzoic acid & & & & & \\
\hline & 417.8 & 20.5 & 0 & 49.07 & 52.2 & 20.5 & 21.8 \\
\hline & & $4 * A 10+2 * A 12+A 36 * B 36+A 45$ & & & & & {$[215]$} \\
\hline \multirow{3}{*}{$\mathrm{C}_{7} \mathrm{H}_{7} \mathrm{NO}_{2}$} & & 3-aminobenzoic acid & & & & & \\
\hline & 452.9 & 21.84 & 0 & 48.12 & 52.2 & 21.84 & 23.6 \\
\hline & & $4 * A 10+2 * A 12+A 36 * B 36+A 45$ & & & & & {$[215]$} \\
\hline \multirow[t]{3}{*}{$\mathrm{C}_{7} \mathrm{H}_{7} \mathrm{NO}_{2}$} & & 4-aminobenzoic acid & & & & & \\
\hline & 461.4 & 20.92 & 0 & 45.19 & 52.2 & 20.92 & 24.1 \\
\hline & & $4 * A 10+2 * A 12+A 36 * B 36+A 45$ & & & & & {$[215]$} \\
\hline \multirow{3}{*}{$\mathrm{C}_{7} \mathrm{H}_{7} \mathrm{NO}_{3}$} & & 4-nitro-5-methylphenol & & & & & \\
\hline & 401 & 27.4 & 0 & 68.33 & 53.3 & 27.4 & 21.4 \\
\hline & & $3 * A 10+A 1+A 11+2 * A 12+A 31+A 50$ & & & & & {$[215]$} \\
\hline \multirow[t]{3}{*}{$\mathrm{C}_{7} \mathrm{H}_{7} \mathrm{NO}_{3}$} & & 2-nitro-5-methylphenol & & & & & \\
\hline & 302.8 & 20.79 & 0 & 68.66 & 53.3 & 20.79 & 16.1 \\
\hline & & $3 * A 10+A 1+A 11+2 * A 12+A 31+A 50$ & & & & & {$[215]$} \\
\hline \multirow{3}{*}{$\mathrm{C}_{7} \mathrm{H}_{7} \mathrm{~N}_{3} \mathrm{O}_{2}$} & & N-acetyl-pyrazinamide & & & & & \\
\hline & 366.7 & 23.6 & 0 & 64.36 & 61.9 & 23.6 & 22.7 \\
\hline & & $3 * A 10+A 12+2 * A 41+A 71+A 1$ & & & & & [9] \\
\hline \multirow[t]{3}{*}{$\mathrm{C}_{7} \mathrm{H}_{8}$} & & toluene & & & & & \\
\hline & 178.0 & 6.62 & 0 & 37.15 & 45.0 & 6.62 & 8.0 \\
\hline & & $5^{*} A 10+A 1+A 11$ & & & & & {$[216]$} \\
\hline \multirow[t]{4}{*}{$\mathrm{C}_{7} \mathrm{H}_{8}$} & & cycloheptatriene & & & & & \\
\hline & 154.0 & 2.35 & 15.24 & & & & \\
\hline & 198.0 & 1.16 & 5.86 & 21.11 & 38.5 & 3.51 & 7.6 \\
\hline & & $A 14+4 * A 15+6 * A 18$ & & & & & {$[216]$} \\
\hline \multirow[t]{4}{*}{$\mathrm{C}_{7} \mathrm{H}_{8}$} & & tetracyclo[3.2.0.0(2,7).0( & leptane & & & & \\
\hline & 180 & 7.2 & 40 & & & & \\
\hline & 228 & 1.09 & 4.8 & 44.8 & 26.6 & 8.29 & 6.1 \\
\hline & & $4 * A 14-5 * A 15+6 * A 16$ & & & & & {$[216]$} \\
\hline \multirow{3}{*}{$\mathrm{C}_{7} \mathrm{H}_{8} \mathrm{~N}_{2} \mathrm{O}$} & & phenylurea & & & & & \\
\hline & 420.6 & 23.68 & 0 & 56.3 & 52.1 & 23.68 & 21.9 \\
\hline & & $5 * A 10+A 12+A 67$ & & & & & {$[215]$} \\
\hline $\mathrm{C}_{7} \mathrm{H}_{8} \mathrm{~N}_{4} \mathrm{O}_{2}$ & & theophylline & & & & & \\
\hline & 544 & 28.2 & 0 & 51.84 & 44.6 & 28.2 & 24.3 \\
\hline & 546.1 & 28.27 & 0 & 51.76 & 44.6 & 28.3 & 24.3 \\
\hline & & $2 * A 14+3 * A 15+2 * A 125+A 118+A 121$ & $A 1+2$ & $8 * B 18$ & & & {$[236,205]$} \\
\hline $\mathrm{C}_{7} \mathrm{H}_{8} \mathrm{O}$ & & benzyl alcohol & & & & & \\
\hline & 257.6 & 8.79 & 0 & 34.11 & 36.4 & 8.79 & 9.4 \\
\hline & & $5^{*} A 10+A 11+A 2+A 30$ & & & & & {$[215]$} \\
\hline $\mathrm{C}_{7} \mathrm{H}_{8} \mathrm{O}$ & & $o$-hydroxytoluene & & & & & \\
\hline & 304.2 & 15.82 & 0 & 52.01 & 50.4 & 15.82 & 15.3 \\
\hline & & $A 31+A 1+A 12+4 * A 10+A 11$ & & & & & {$[216]$} \\
\hline $\mathrm{C}_{7} \mathrm{H}_{8} \mathrm{O}$ & & $m$-hydroxytoluene & & & & & \\
\hline & 285.4 & 10.71 & 0 & 37.53 & 50.42 & 10.71 & 14.39 \\
\hline & & $A 31+A 1+A 12+4 * A 10+A 11$ & & & & & {$[216]$} \\
\hline $\mathrm{C}_{7} \mathrm{H}_{8} \mathrm{O}$ & & $p$-hydroxytoluene & & & & & \\
\hline & 307.9 & 12.72 & 0 & 41.25 & 50.42 & 12.72 & 15.52 \\
\hline & & $A 31+A 1+A 12+4 * A 10+A 11$ & & & & & {$[216]$} \\
\hline $\mathrm{C}_{7} \mathrm{H}_{8} \mathrm{O}$ & & methoxybenzene & & & & & \\
\hline & 268.7 & 12.9 & 0 & 48.0 & 51.9 & 12.9 & 13.9 \\
\hline & & $5 * A 10+A 12+A 1+A 32$ & & & & & {$[216]$} \\
\hline $\mathrm{C}_{7} \mathrm{H}_{8} \mathrm{~S}$ & & methylphenylsulfide & & & & & \\
\hline & 256.4 & 14.85 & 0 & 57.86 & 49.3 & 14.85 & 12.63 \\
\hline & & $5 * A 10+A 12+A 1+A 84$ & & & & & {$[105]$} \\
\hline $\mathrm{C}_{7} \mathrm{H}_{9} \mathrm{Cl}_{3} \mathrm{NO}$ & & O,O-dimethyl-O- $(3,5,6-\mathrm{tr}$ & ro-2-pyi & phorothioate & & & \\
\hline & 318.7 & 25.92 & 0 & 81.32 & 73.2 & 25.92 & 23.3 \\
\hline & & $4 * A 12+A 10+A 41+3 * A 22^{*} E 22+2 * A$ & & & & & {$[221]$} \\
\hline $\mathrm{C}_{9} \mathrm{H}_{13} \mathrm{Cl}_{3} \mathrm{NC}$ & & O,O-diethyl-O-(3,5,6-tric & -2-pyric & late & & & \\
\hline & 312.5 & 15.61 & 0 & 49.97 & 76.4 & 15.61 & 23.9 \\
\hline & & $4^{*} A 12+A 10+A 41+3 * A 22^{*} E 22+2 * A$ & $74+2 *$ & & & & {$[221]$} \\
\hline $\mathrm{C}_{7} \mathrm{H}_{9} \mathrm{~N}$ & & $m$-toluidine & & & & & \\
\hline & 241.7 & 8.8 & 0 & 36.41 & 51.5 & 8.8 & 12.5 \\
\hline & & $A 45+4 * A 10+A 12+A 1+A 11$ & & & & & {$[215]$} \\
\hline $\mathrm{C}_{7} \mathrm{H}_{9} \mathrm{~N}$ & & $p$-toluidine & & & & & \\
\hline & 316.5 & 17.89 & 0 & 56.52 & 51.5 & 17.89 & 16.3 \\
\hline & & $A 45+4 * A 10+A 12+A 1+A 11$ & & & & & {$[215]$} \\
\hline
\end{tabular}


Table 5. Experimental and calculated total phase change enthalpy and entropy of database-Continued

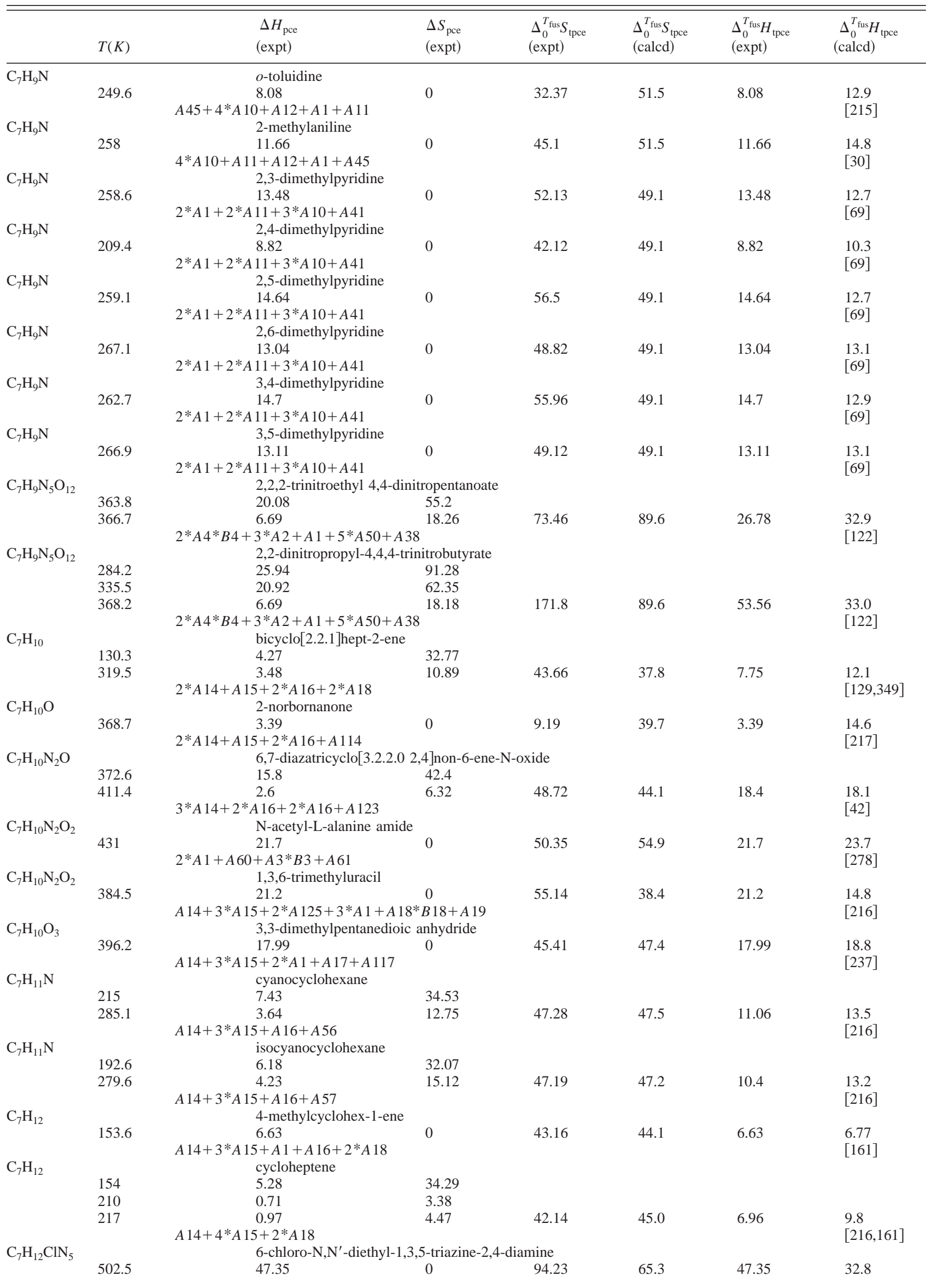


Table 5. Experimental and calculated total phase change enthalpy and entropy of database-Continued

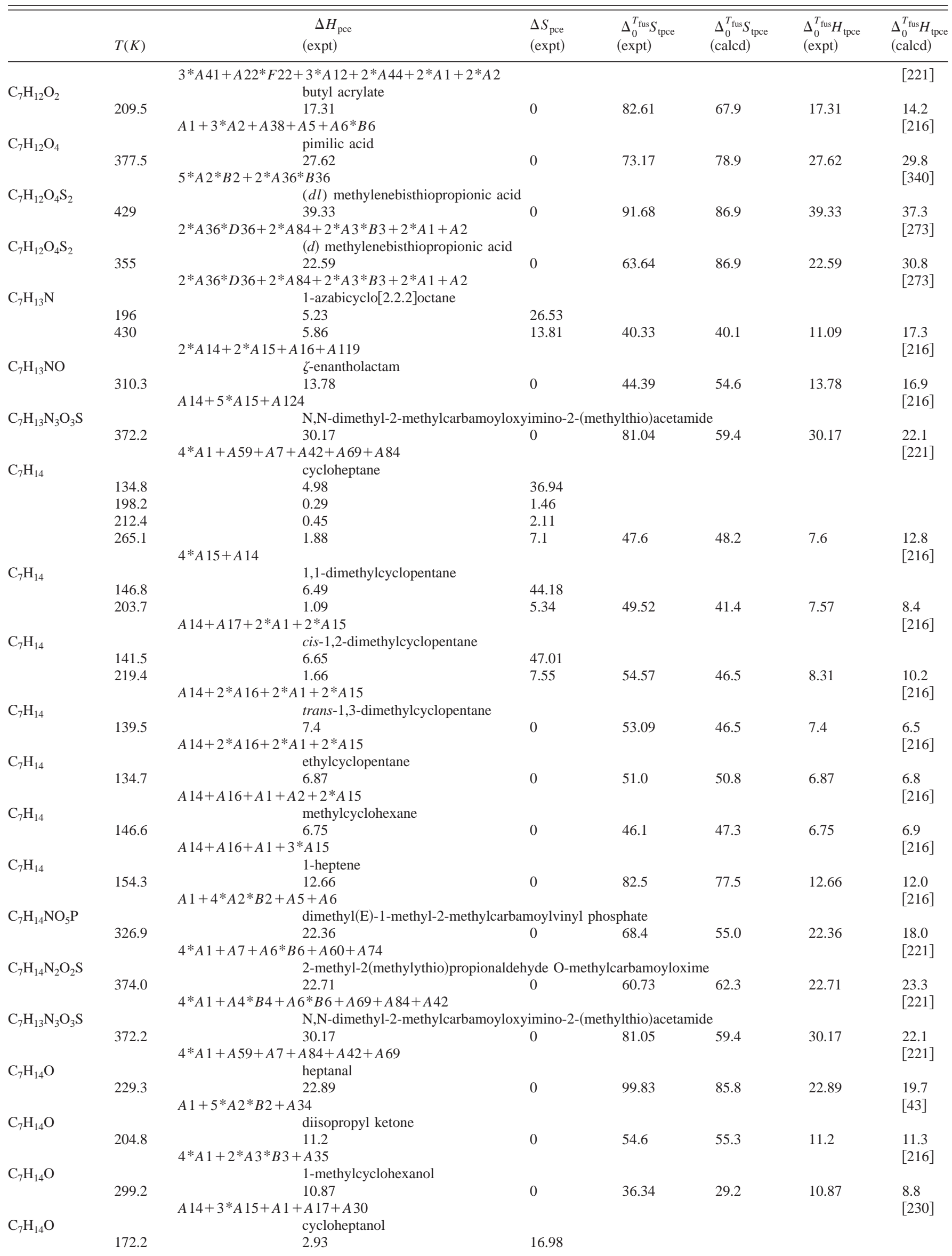


Table 5. Experimental and calculated total phase change enthalpy and entropy of database-Continued

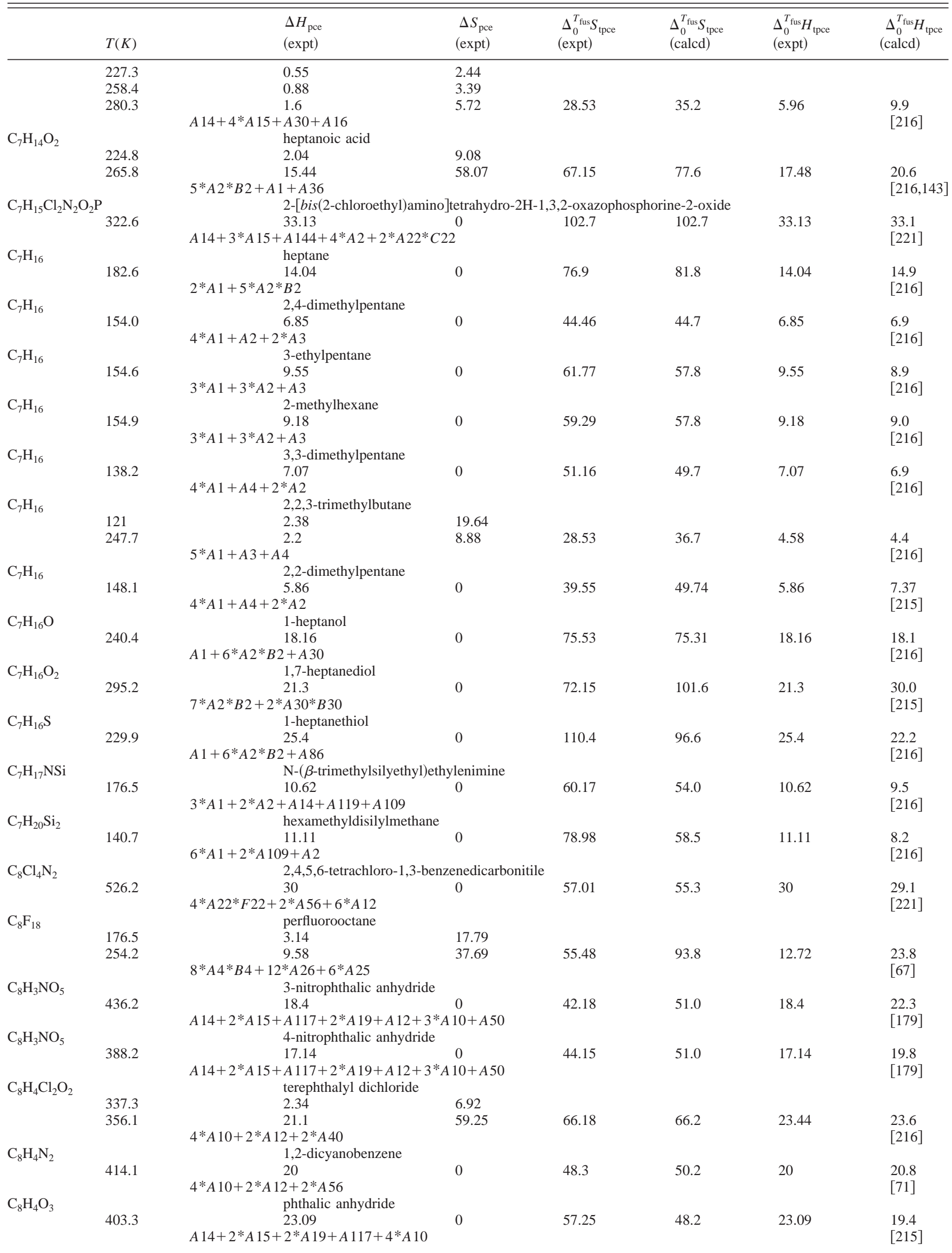


Table 5. Experimental and calculated total phase change enthalpy and entropy of database-Continued

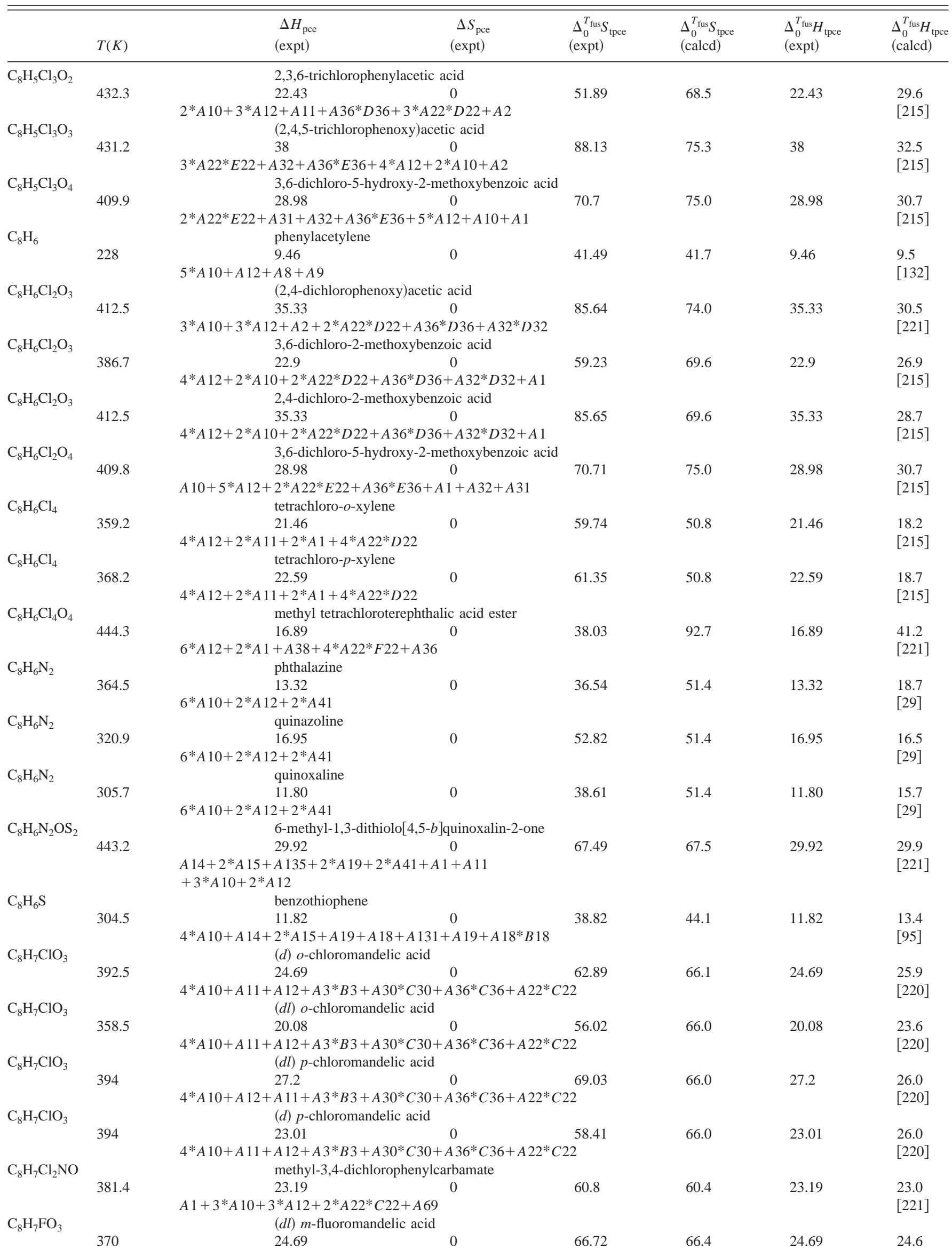


Table 5. Experimental and calculated total phase change enthalpy and entropy of database-Continued

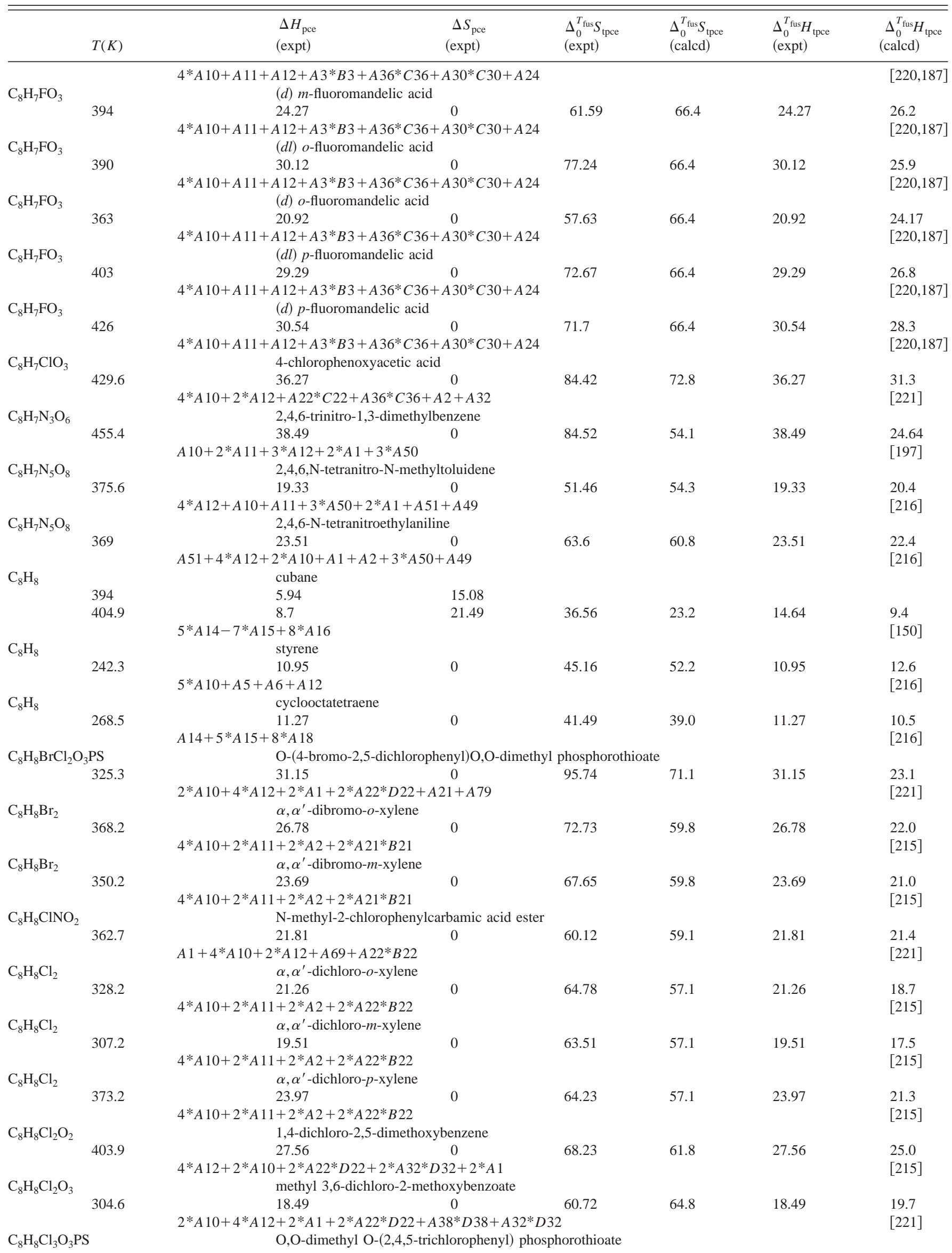


Table 5. Experimental and calculated total phase change enthalpy and entropy of database-Continued

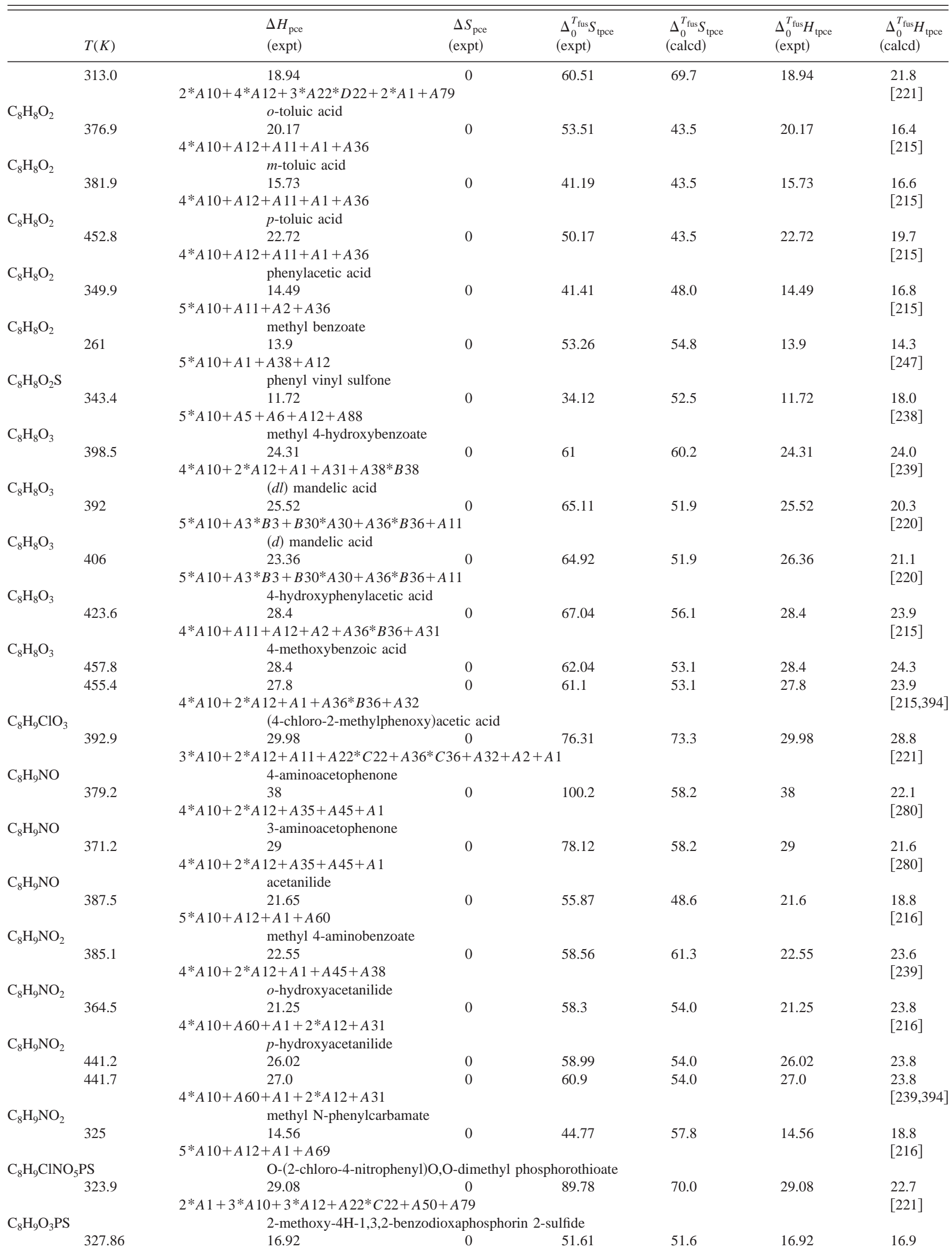


Table 5. Experimental and calculated total phase change enthalpy and entropy of database-Continued

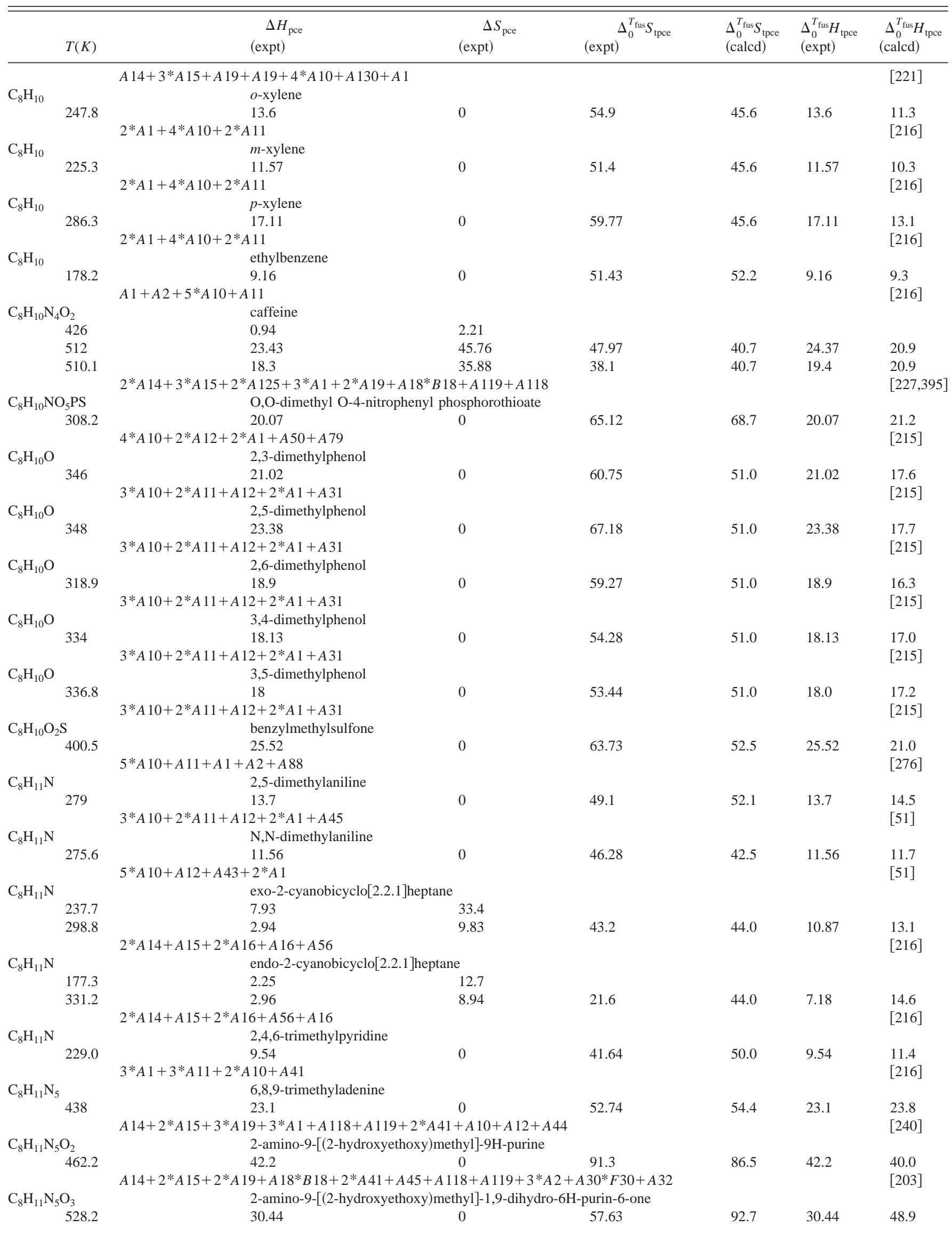


Table 5. Experimental and calculated total phase change enthalpy and entropy of database-Continued

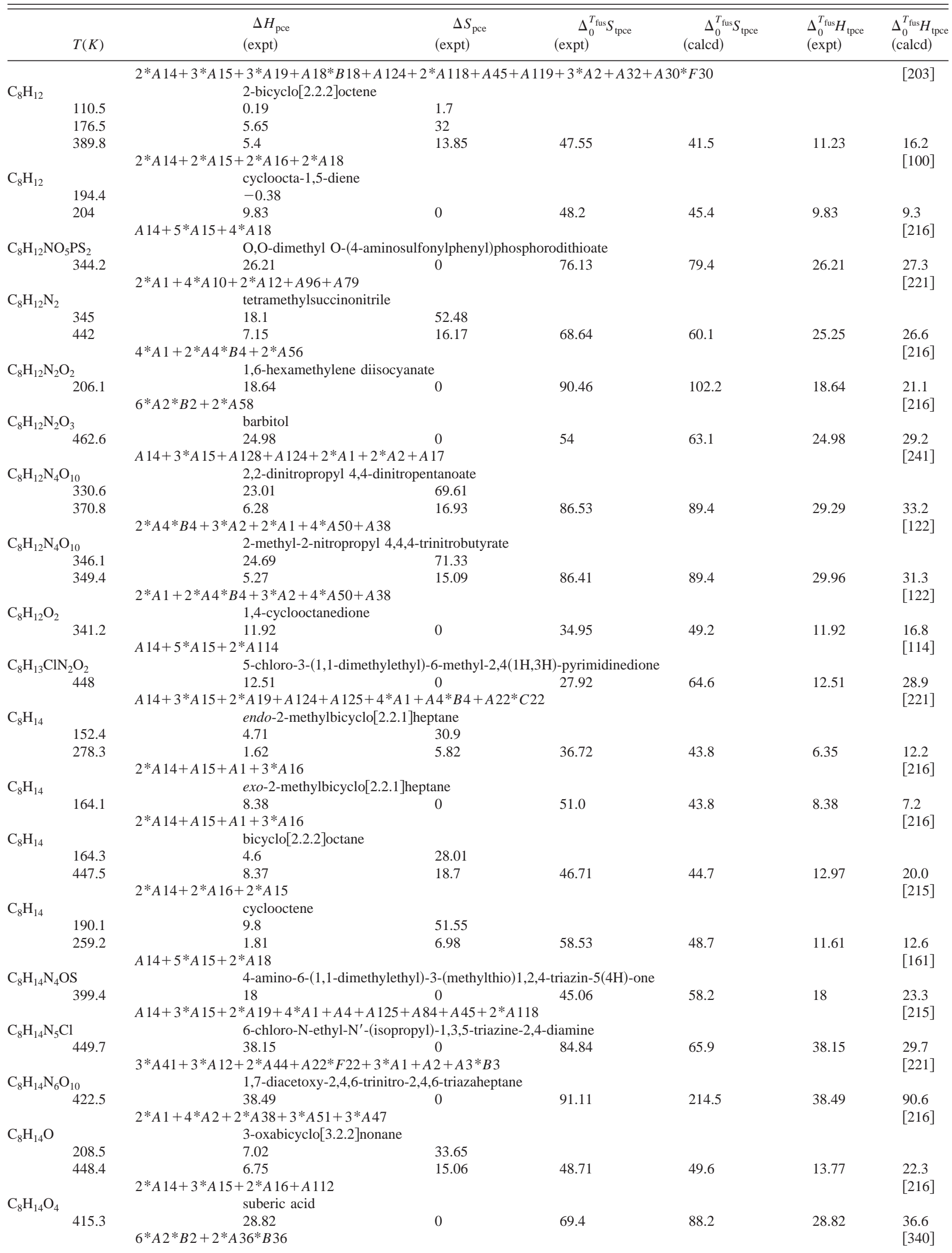


Table 5. Experimental and calculated total phase change enthalpy and entropy of database-Continued

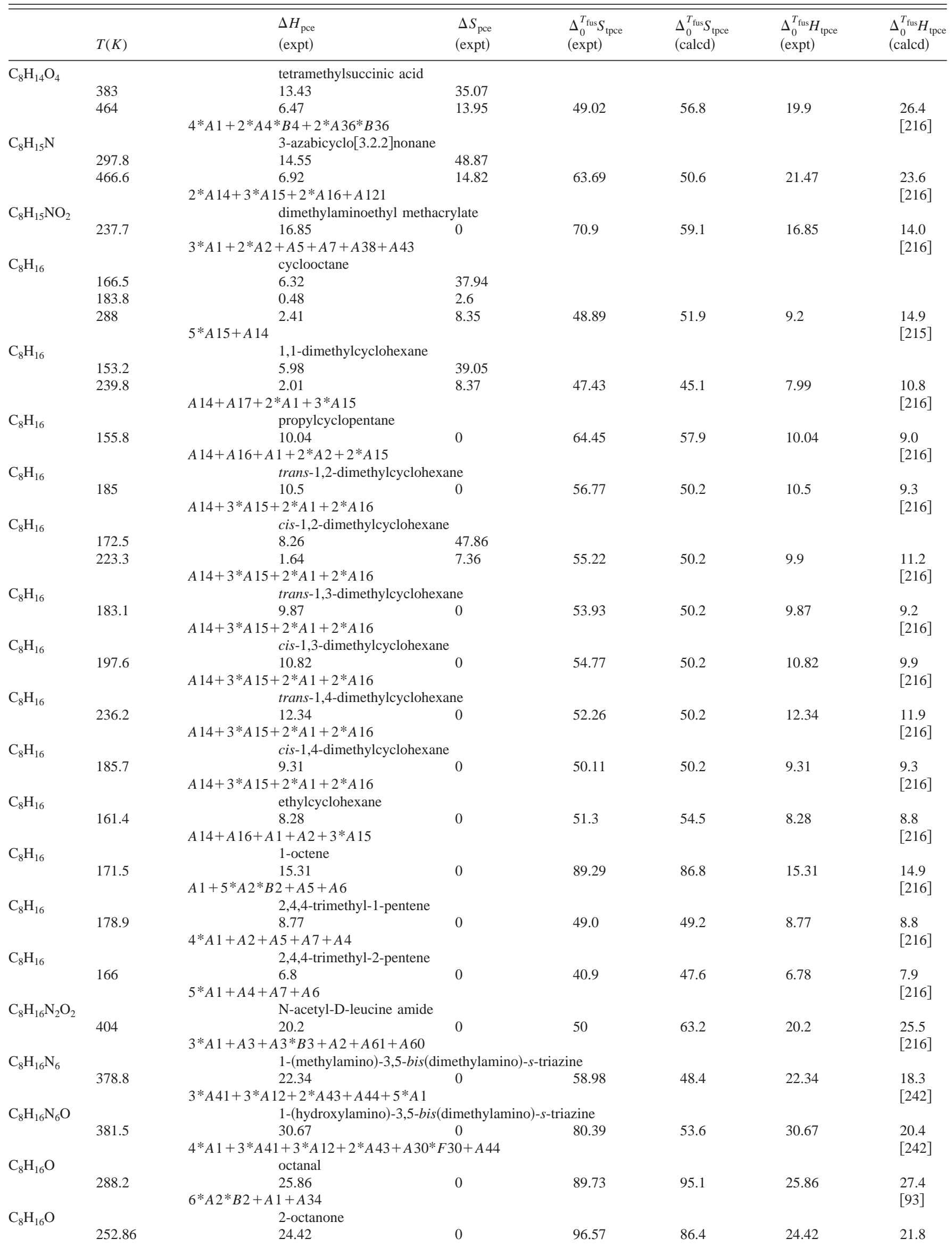


Table 5. Experimental and calculated total phase change enthalpy and entropy of database-Continued

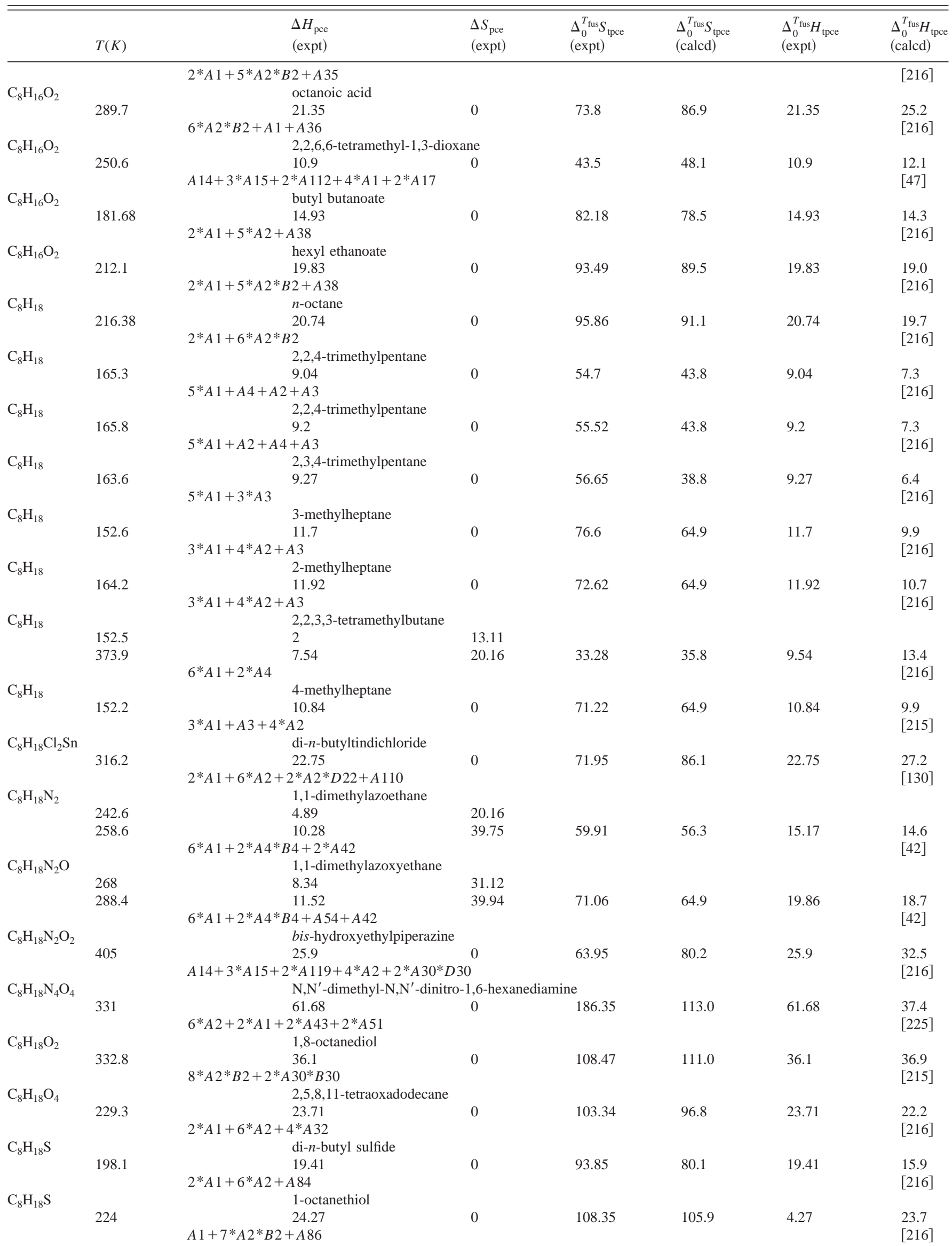


Table 5. Experimental and calculated total phase change enthalpy and entropy of database-Continued

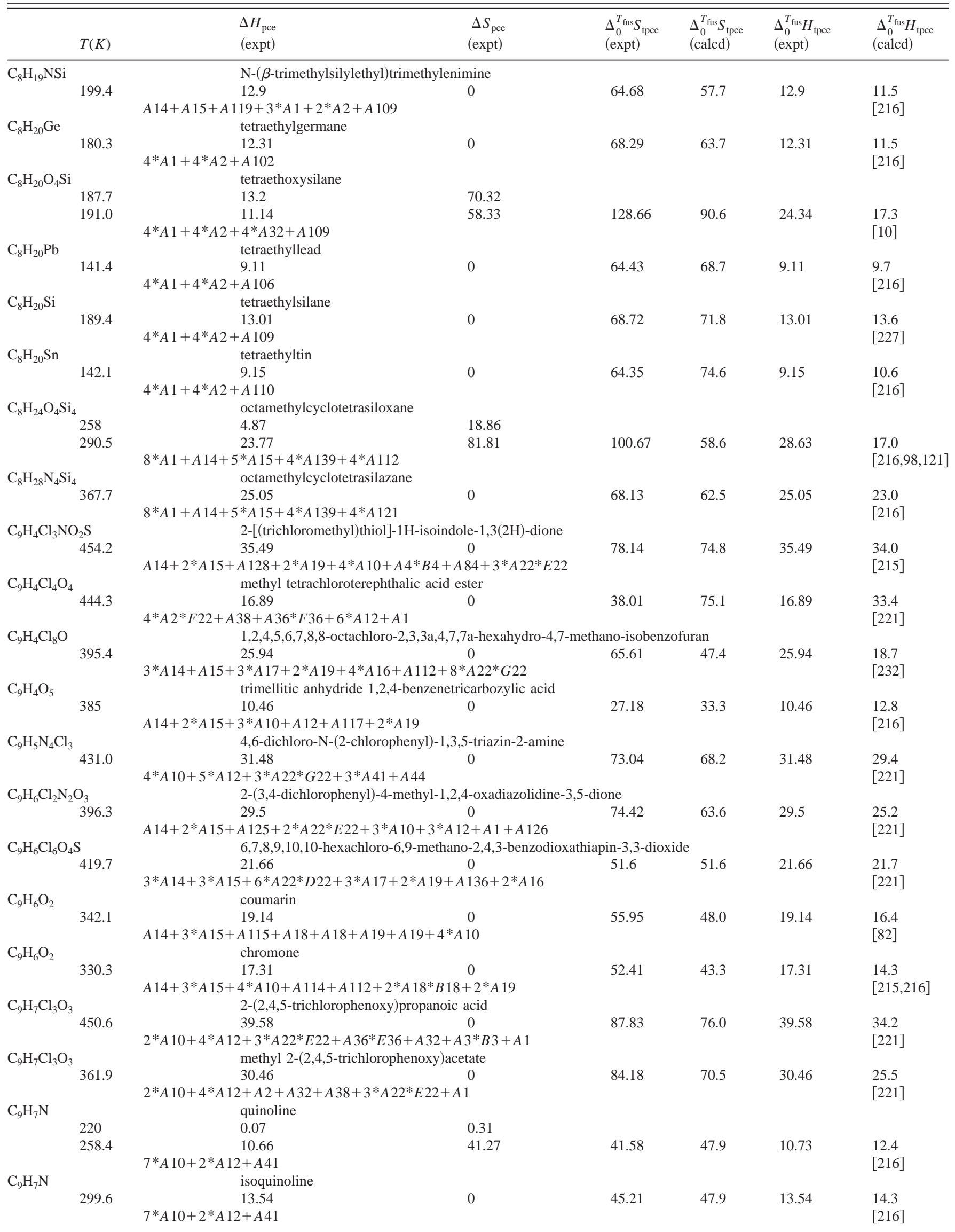


Table 5. Experimental and calculated total phase change enthalpy and entropy of database-Continued

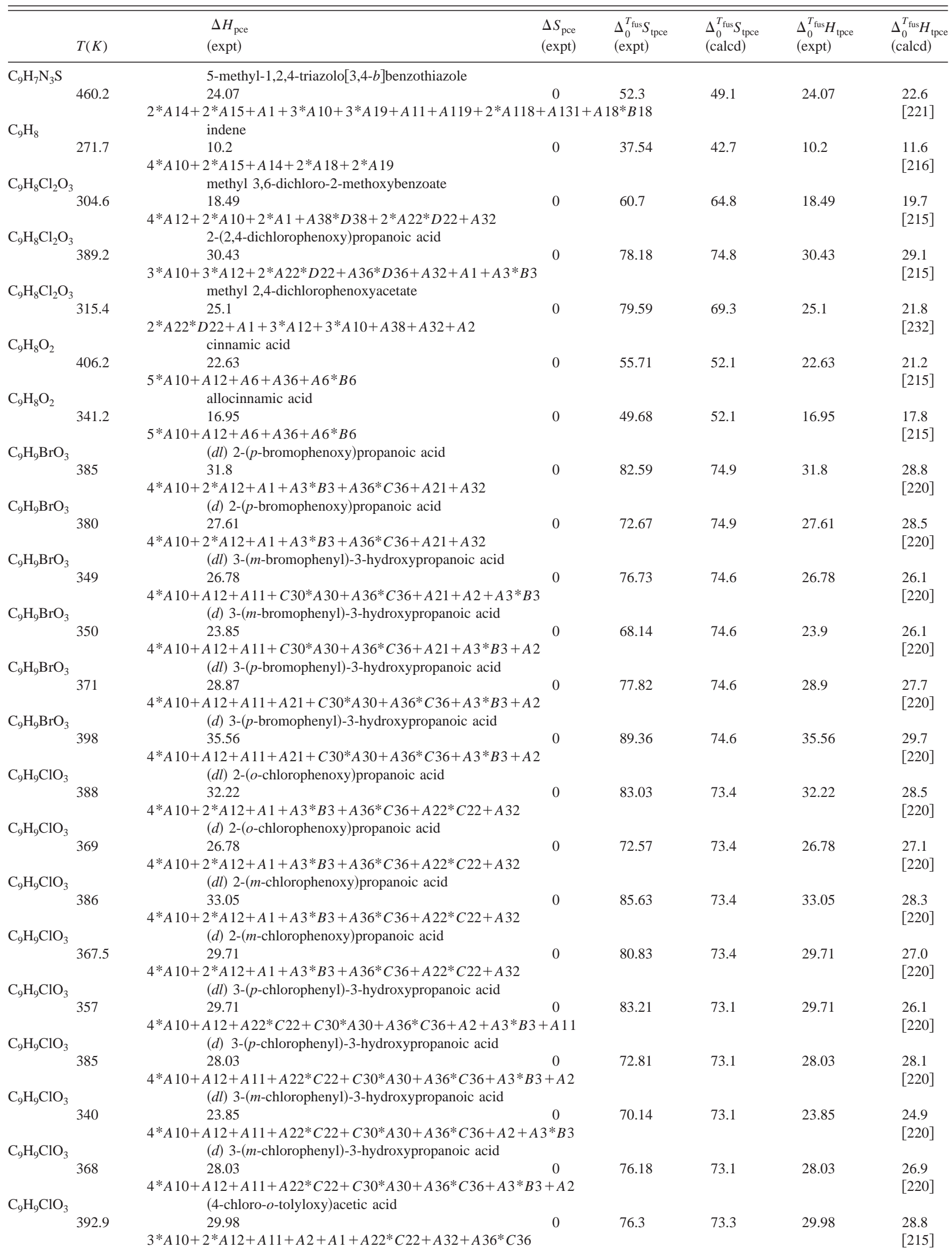


Table 5. Experimental and calculated total phase change enthalpy and entropy of database-Continued

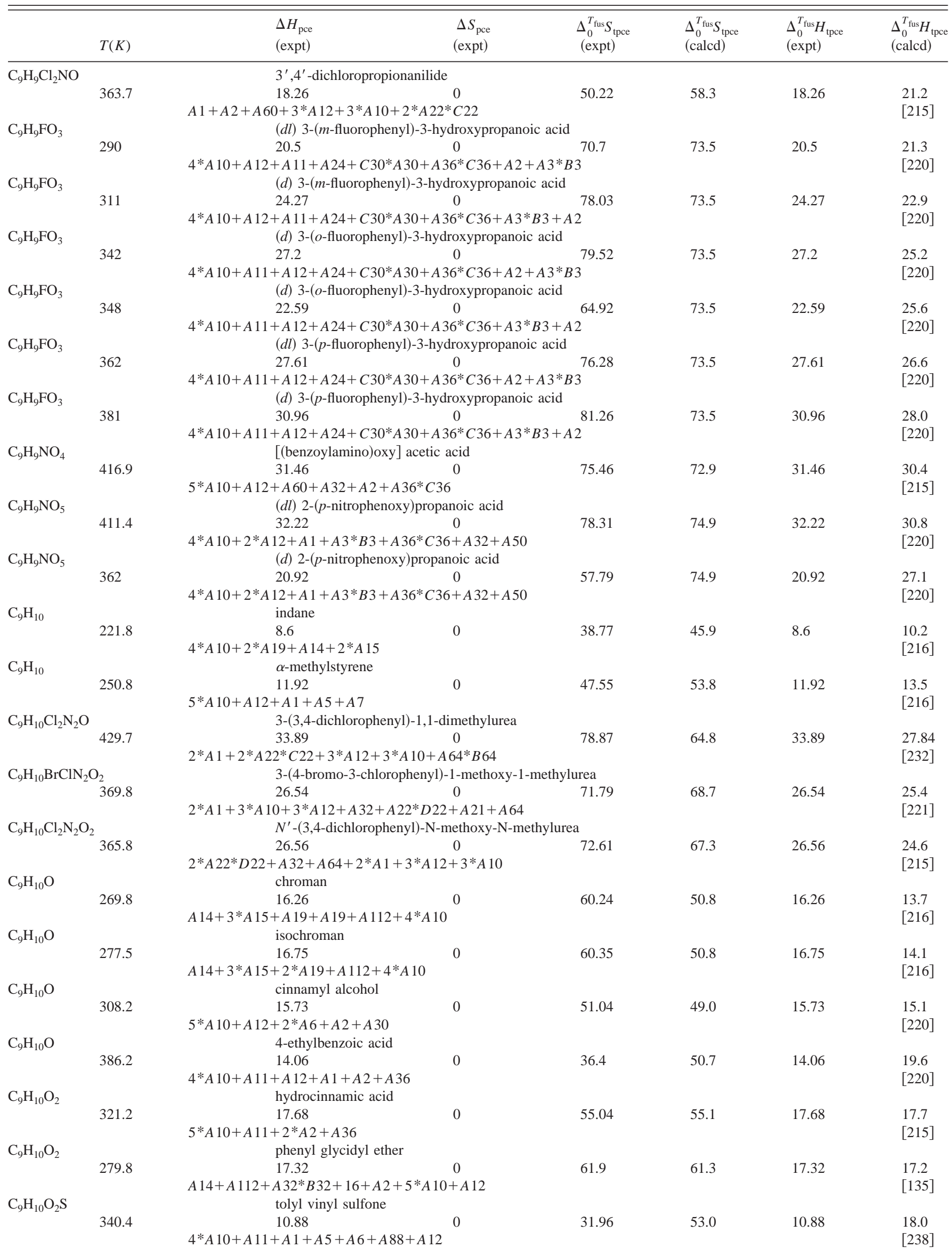


Table 5. Experimental and calculated total phase change enthalpy and entropy of database-Continued

\begin{tabular}{|c|c|c|c|c|c|c|}
\hline$T(K)$ & $\begin{array}{l}\Delta H_{\mathrm{pce}} \\
(\text { expt) }\end{array}$ & $\begin{array}{l}\Delta S_{\mathrm{pce}} \\
(\operatorname{expt})\end{array}$ & $\begin{array}{l}\Delta_{0}^{T_{\text {fus }}} S_{\text {tpce }} \\
(\operatorname{expt})\end{array}$ & $\begin{array}{l}\Delta_{0}^{T_{\text {fus }}} S_{\text {tpce }} \\
\text { (calcd) }\end{array}$ & $\begin{array}{l}\Delta_{0}^{T_{\text {fus }}} H_{\text {tpce }} \\
\text { (expt) }\end{array}$ & $\begin{array}{l}\Delta_{0}^{T_{\text {fus }}} H_{\text {tpce }} \\
\text { (calcd) }\end{array}$ \\
\hline \multirow[t]{3}{*}{$\mathrm{C}_{9} \mathrm{H}_{10} \mathrm{O}_{3}$} & (dl) 3-phenyl-3-hydroxypropanoic acid & & & & & \\
\hline & 29.71 & 0 & 81.17 & 59.2 & 29.71 & 21.7 \\
\hline & $5 * A 10+A 11+A 2+A 3 * B 3+B 30 * A 30+A 36 * B 36$ & & & & & {$[220]$} \\
\hline \multirow{3}{*}{$\mathrm{C}_{9} \mathrm{H}_{10} \mathrm{O}_{3}$} & (d) 3-phenyl-3-hydroxypropanoic acid & & & & & \\
\hline & 32.64 & 0 & 83.47 & 59.2 & 32.64 & 23.1 \\
\hline & $5 * A 10+A 11+A 2+A 3 * B 3+A 30 * B 30 *+B 36 * A 36$ & & & & & {$[220]$} \\
\hline \multirow{3}{*}{$\mathrm{C}_{9} \mathrm{H}_{10} \mathrm{O}_{3}$} & (dl) 2-phenoxypropionic acid & & & & & \\
\hline & 33.05 & 0 & 85.19 & 58.2 & 33.05 & 22.6 \\
\hline & $5 * A 10+A 12+A 1+A 3 * B 3+A 36 * B 36+A 32 * B 32$ & & & & & {$[220]$} \\
\hline \multirow{3}{*}{$\mathrm{C}_{9} \mathrm{H}_{10} \mathrm{O}_{3}$} & (d) 2-phenoxypropionic acid & & & & & \\
\hline & 22.59 & 0 & 62.93 & 58.2 & 22.59 & 20.9 \\
\hline & $5 * A 10+A 12+A 1+A 3 * B 3+A 36 * B 36+A 32 * B 32$ & & & & & {$[220]$} \\
\hline \multirow{3}{*}{$\mathrm{C}_{9} \mathrm{H}_{10} \mathrm{O}_{3}{ }_{358}$} & 4-methoxyphenylacetic acid & & & & & \\
\hline & 21.8 & 0 & 60.88 & 58.1 & 21.8 & 20.8 \\
\hline & $4 * A 10+A 11+A 12+A 1+A 2+A 36 * B 36+A 32 * B 32$ & & & & & {$[215]$} \\
\hline \multirow[t]{3}{*}{$\mathrm{C}_{9} \mathrm{H}_{10} \mathrm{O}_{3}$} & 4-hydroxyphenylpropionic acid & & & & & \\
\hline & 28.9 & 0 & 71.8 & 63.2 & 28.9 & 25.5 \\
\hline & $4 * A 10+A 11+A 12+2 * A 2+A 36 * B 36+A 31$ & & & & & {$[215]$} \\
\hline \multirow[t]{3}{*}{$\mathrm{C}_{9} \mathrm{H}_{10} \mathrm{O}_{3}$} & 4-ethoxybenzoic acid & & & & & \\
\hline & 29.4 & 0 & 62.18 & 60.2 & 29.4 & 28.5 \\
\hline & $4 * A 10+2 * A 12+A 1+A 2+A 36 * B 36+A 32 * B 32$ & & & & & {$[215]$} \\
\hline \multirow{3}{*}{$\mathrm{C}_{9} \mathrm{H}_{10} \mathrm{O}_{4}$} & $(d l)$ erythro phenylglyceric acid & & & & & \\
\hline & 31.38 & 0 & 79.44 & 71.9 & 31.38 & 28.4 \\
\hline & $5 * A 10+A 11+2 * A 3 * B 3+2 * C 30 * A 30+A 36 * C 36$ & & & & & {$[220]$} \\
\hline \multirow{3}{*}{$\mathrm{C}_{9} \mathrm{H}_{10} \mathrm{O}_{4}$} & $(d)$ erythro phenylglyceric acid & & & & & \\
\hline & 23.43 & 0 & 63.07 & 71.9 & 23.43 & 26.7 \\
\hline & $5 * A 10+A 11+2 * A 3 * B 3+2 * C 30 * A 30+A 36 * C 36$ & & & & & {$[220]$} \\
\hline \multirow{3}{*}{$\begin{array}{r}\mathrm{C}_{9} \mathrm{H}_{11} \mathrm{BrN}_{2} \mathrm{O} \\
368.3\end{array}$} & $\mathrm{~N}^{\prime}$-(4-bromophenyl)-N-methoxy-N-methyl urea & & & & & \\
\hline & 24.44 & 0 & 66.36 & 67.4 & 24.44 & 24.8 \\
\hline & $2 * A 1+A 32 * C 32+4 * A 10+2 * A 12+A 64 * C 64 *+A 21$ & & & & & {$[215]$} \\
\hline \multirow{3}{*}{$\begin{array}{r}\mathrm{C}_{9} \mathrm{H}_{11} \mathrm{ClN}_{2} \mathrm{O}_{2} \\
353.4\end{array}$} & $\mathrm{~N}^{\prime}$-(4-chlorophenyl)-N-methoxy-N-methyl urea & & & & & \\
\hline & 22.54 & 0 & 63.78 & 66.0 & 22.54 & 23.3 \\
\hline & $2 * A 1+4 * A 10+2 * A 12+A 22 * C 22+A 64 * C 64+A 32$ & & & & & {$[215]$} \\
\hline \multirow{3}{*}{$\begin{array}{r}\mathrm{C}_{9} \mathrm{H}_{11} \mathrm{ClN}_{2} \mathrm{O} \\
447.6\end{array}$} & 3-(4-chlorophenyl)-1,1-dimethyl urea & & & & & \\
\hline & 29.46 & 0 & 65.82 & 66.0 & 29.46 & 29.6 \\
\hline & $2 * A 1+A 64 * B 64+A 22 * B 22+2 * A 12+4 * A 10$ & & & & & {$[215]$} \\
\hline $\mathrm{C}_{9} \mathrm{H}_{11} \mathrm{ClO}_{3}$ & 2-(4-chloro-2-methylphenoxy)propanoic acid & & & & & \\
\hline 366.2 & 26.43 & 0 & 72.16 & 73.9 & 26.43 & 27.1 \\
\hline & $3 * A 10+2 * A 12+A 11+A 22 * C 22+A 36 * C 36+A 32 * C 32+A 3 * B 3+2 * A 1$ & & & & & {$[221]$} \\
\hline $\mathrm{C}_{9} \mathrm{H}_{11} \mathrm{Cl}_{3} \mathrm{NO}_{3} \mathrm{PS}$ & O,O-diethyl-O-(3,5,6-trichloro-2-pyridyl)phosphorothioate & & & & & \\
\hline 315 & 24.53 & 0 & 77.86 & 87.5 & 24.53 & 27.6 \\
\hline & $4 * A 12+A 10+A 41+3 * A 22 * E 22+2 * A 1+2 * A 2+A 79$ & & & & & {$[215]$} \\
\hline $\mathrm{C}_{9} \mathrm{H}_{11} \mathrm{~N}$ & 1,2,3,4-tetrahydroquinoline & & & & & \\
\hline 290 & 11.81 & 0 & 40.73 & 51.8 & 11.81 & 15.0 \\
\hline & $A 14+3 * A 15+A 121+A 19+4 * A 10+A 19$ & & & & & {$[215]$} \\
\hline $\mathrm{C}_{9} \mathrm{H}_{11} \mathrm{~N}$ & 5,6,7,8-tetrahydroquinoline & & & & & \\
\hline 222.7 & 9.08 & 0 & 40.75 & 53.1 & 9.08 & 11.8 \\
\hline & $A 14+3 * A 15+3 * A 10+A 41+A 19+A 19$ & & & & & {$[215]$} \\
\hline $\mathrm{C}_{9} \mathrm{H}_{11} \mathrm{NO}_{2}$ & ethyl phenyl carbamate & & & & & \\
\hline 326 & 16.27 & 0 & 49.79 & 64.9 & 16.27 & 21.2 \\
\hline & $5 * A 10+A 12+A 1+A 2+A 69$ & & & & & {$[102]$} \\
\hline $\mathrm{C}_{9} \mathrm{H}_{11} \mathrm{NO}_{2}$ & ethyl 4-aminobenzoate & & & & & \\
\hline 362.8 & 23.56 & 0 & 64.94 & 68.5 & 23.56 & 24.8 \\
\hline 363.2 & 22.0 & 0 & 60.6 & 68.5 & 22.0 & 24.8 \\
\hline & $4 * A 10+2 * A 12+A 1+A 2+A 38+A 5$ & & & & & {$[215,395]$} \\
\hline $\mathrm{C}_{9} \mathrm{H}_{11} \mathrm{NO}_{2}$ & $p$-methoxyacetanilide & & & & & \\
\hline 400.3 & 27.82 & 0 & 69.51 & 56.0 & 27.82 & 22.4 \\
\hline & $2 * A 1+4 * A 10+2 * A 12+A 32+A 60$ & & & & & {$[239]$} \\
\hline $\mathrm{C}_{9} \mathrm{H}_{12}$ & 1,2,3-trimethylbenzene & & & & & \\
\hline 218.7 & 0.66 & 3 & & & & \\
\hline 230.3 & 1.33 & 5.8 & & & & \\
\hline 247.8 & 8.18 & 33.01 & 41.81 & 46.2 & 10.17 & 11.4 \\
\hline & $3 * A 1+3 * A 10+3 * A 11$ & & & & & {$[216]$} \\
\hline $\mathrm{C}_{9} \mathrm{H}_{12}$ & 1,2,4-trimethylbenzene & & & & & \\
\hline 229.3 & 13.19 & 0 & 57.53 & 46.2 & 13.19 & 10.59 \\
\hline & $3 * A 1+3 * A 10+3 * A 11$ & & & & & {$[216]$} \\
\hline
\end{tabular}


Table 5. Experimental and calculated total phase change enthalpy and entropy of database-Continued

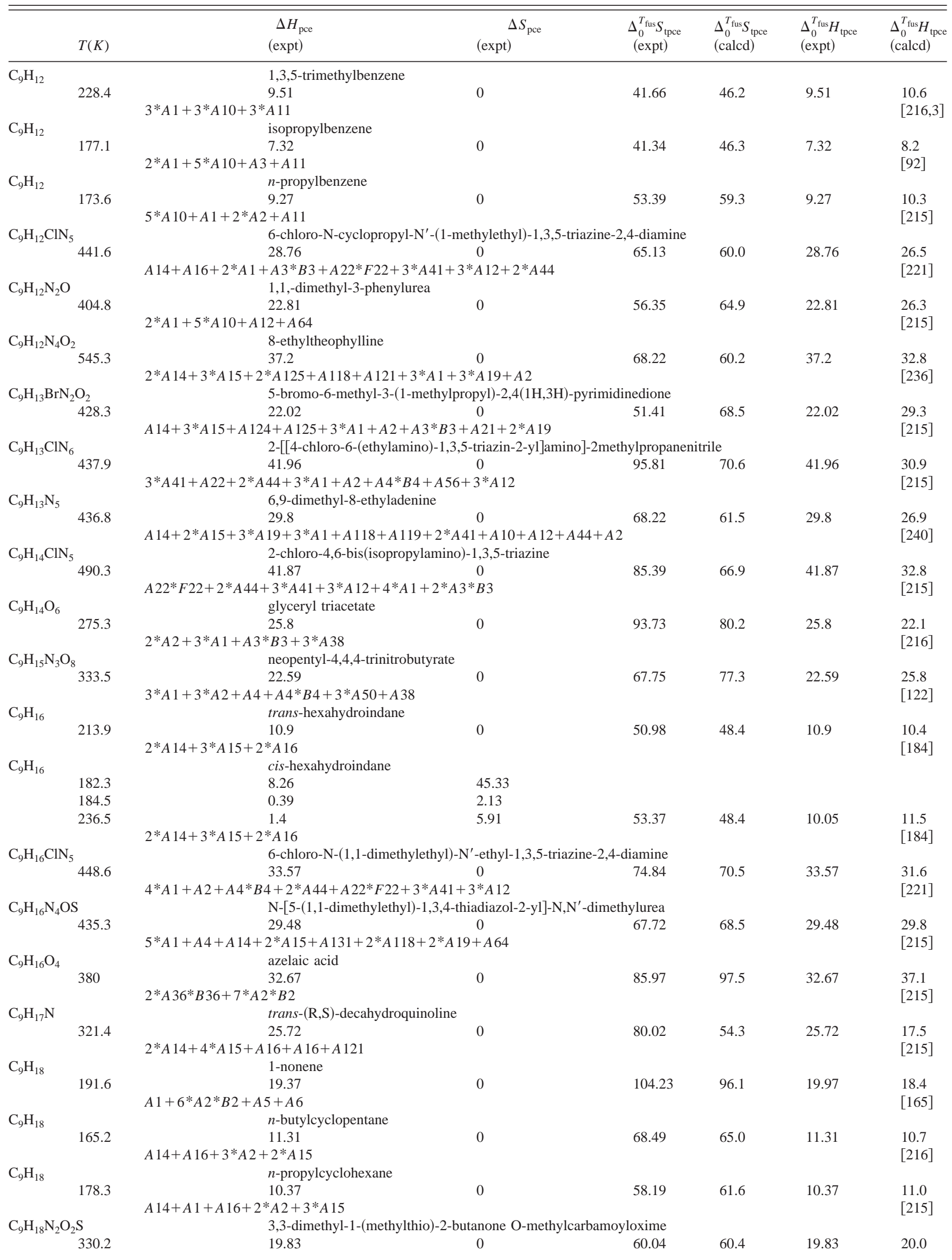


Table 5. Experimental and calculated total phase change enthalpy and entropy of database-Continued

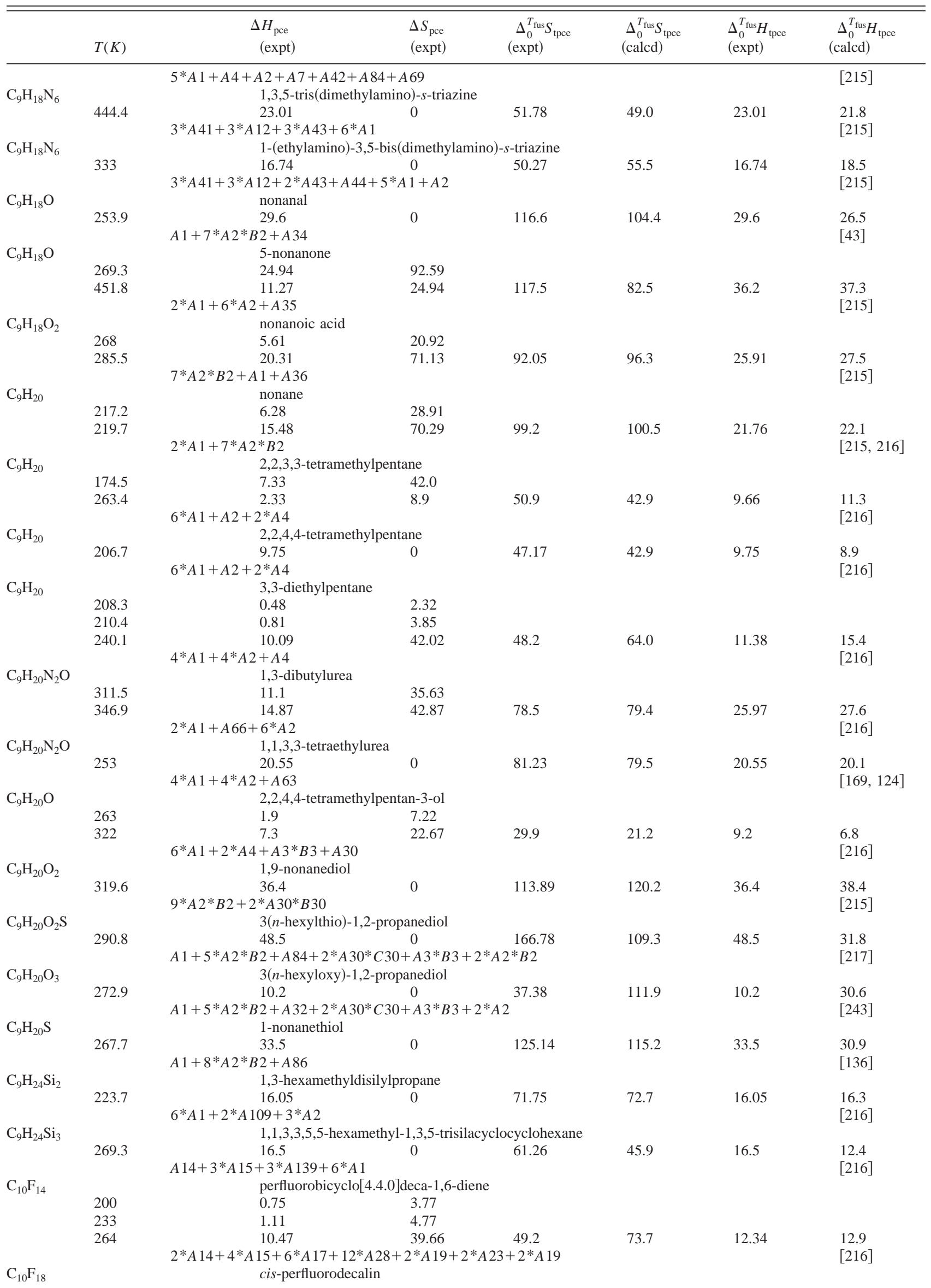


Table 5. Experimental and calculated total phase change enthalpy and entropy of database-Continued

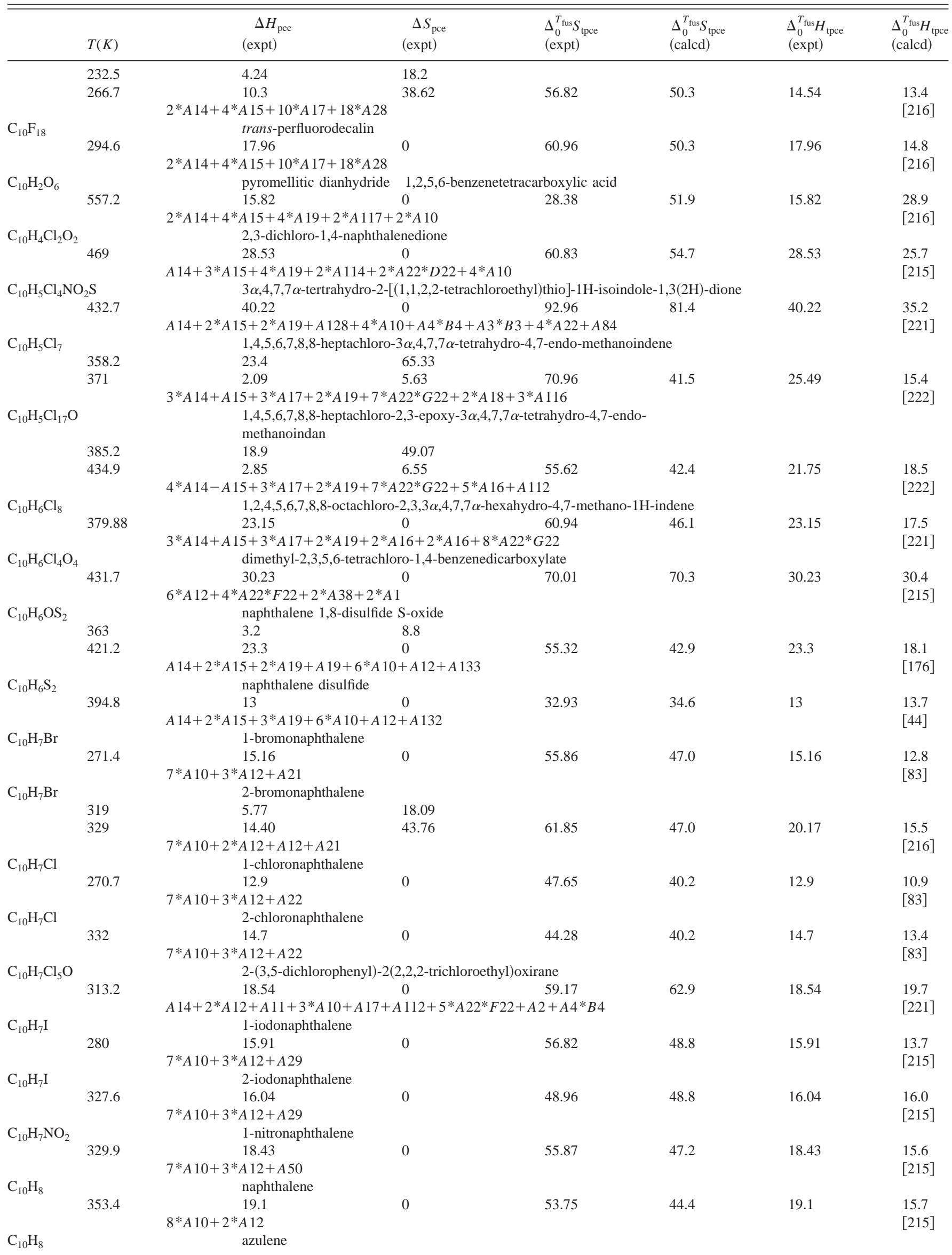


Table 5. Experimental and calculated total phase change enthalpy and entropy of database-Continued

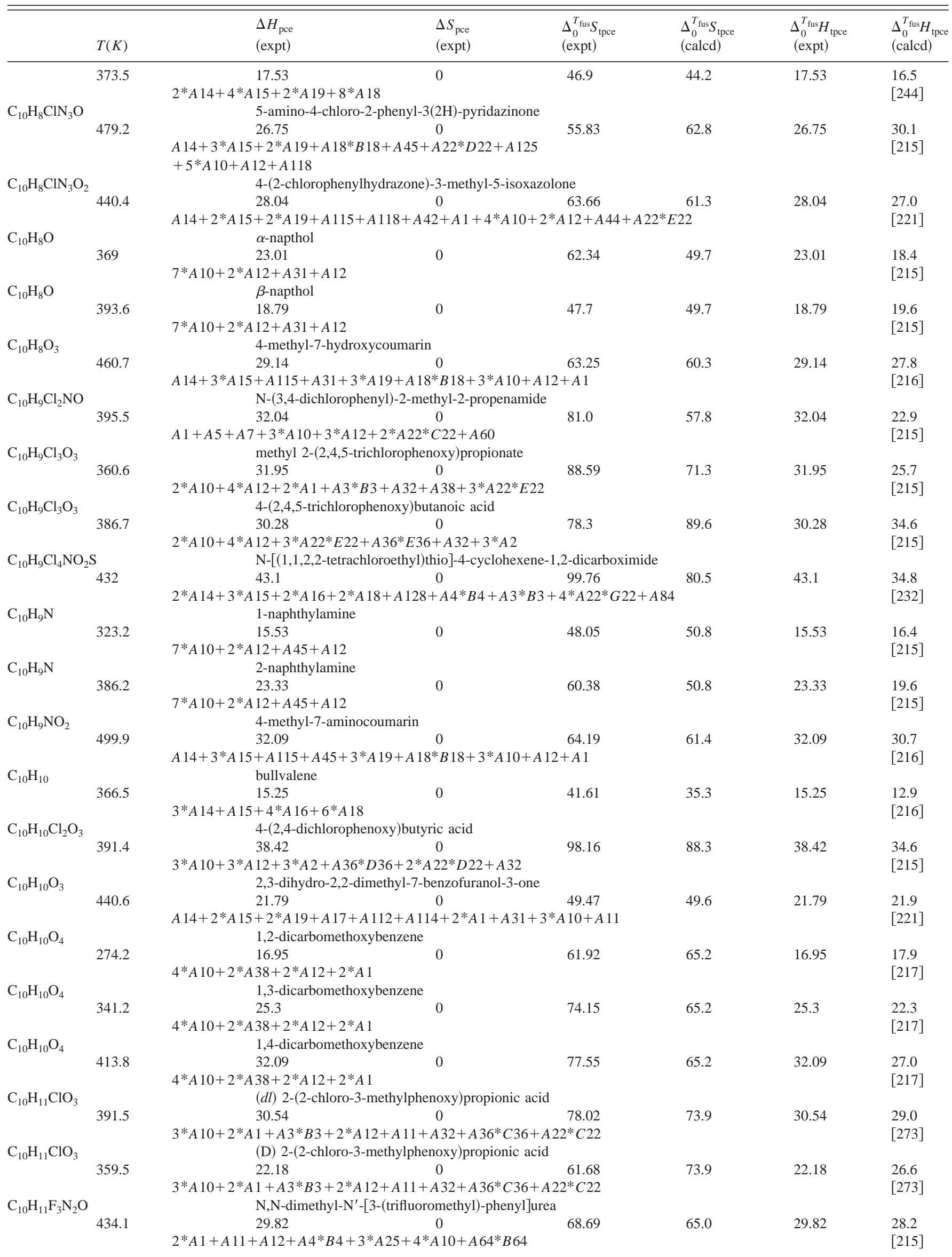


Table 5. Experimental and calculated total phase change enthalpy and entropy of database-Continued

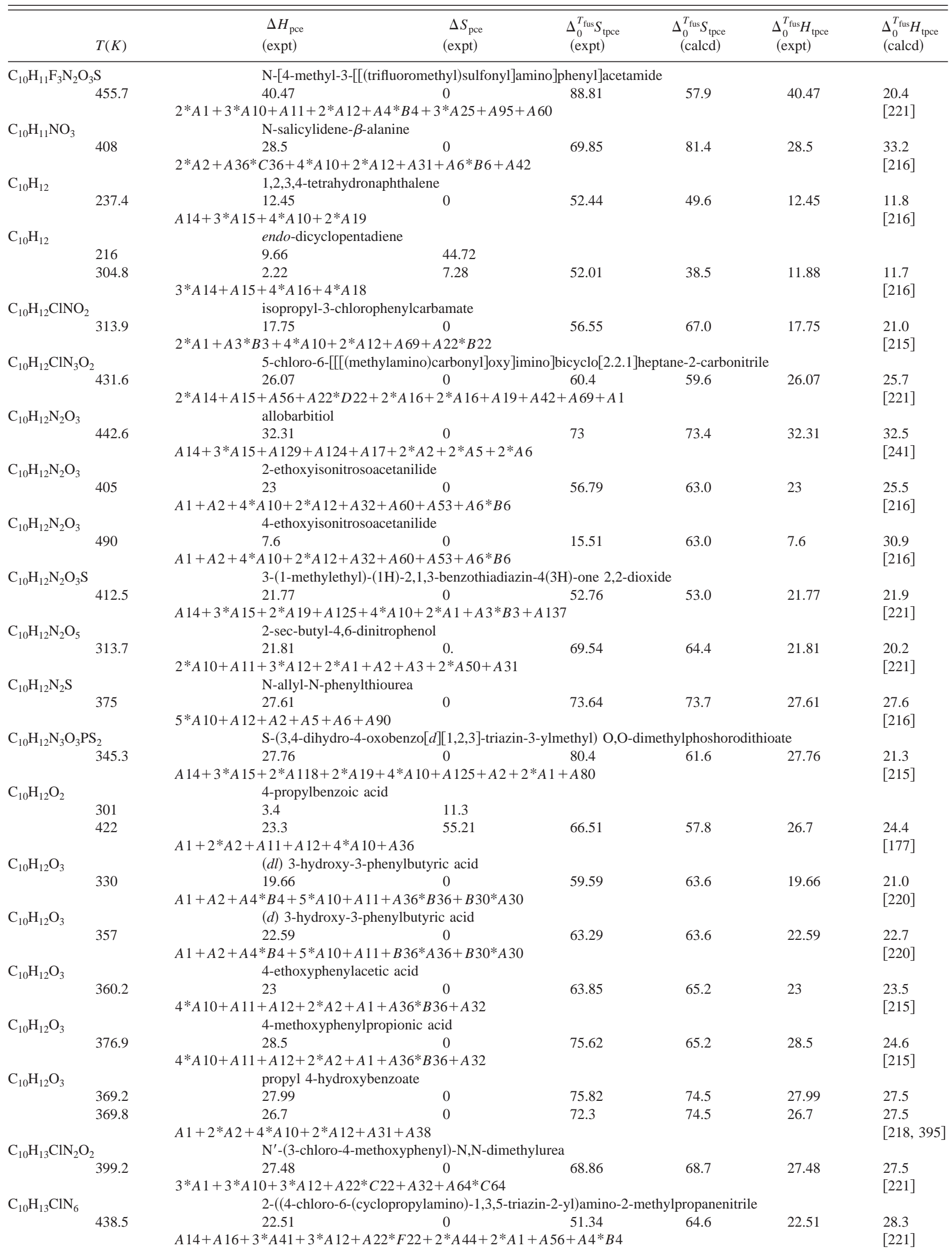


Table 5. Experimental and calculated total phase change enthalpy and entropy of database-Continued

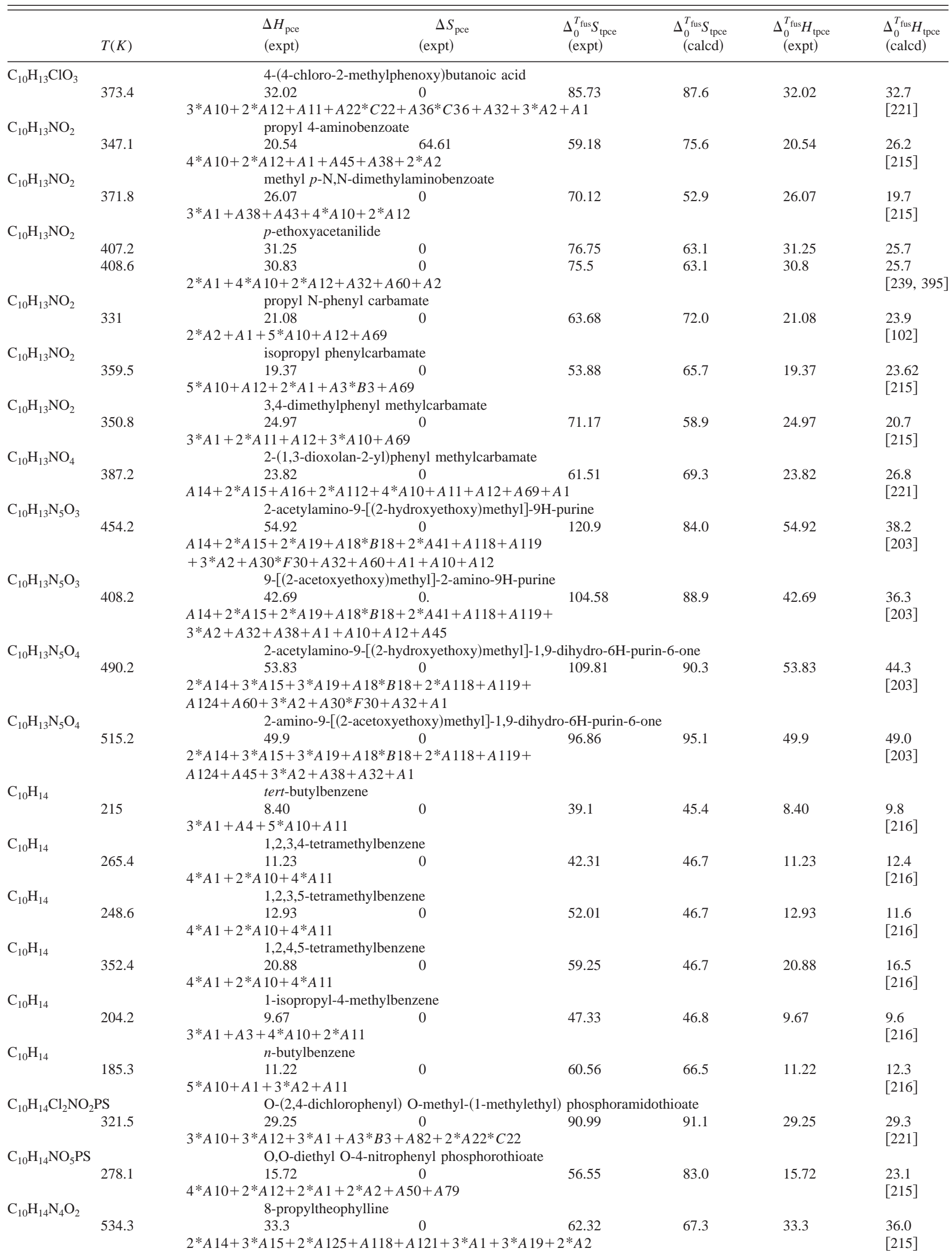


Table 5. Experimental and calculated total phase change enthalpy and entropy of database-Continued

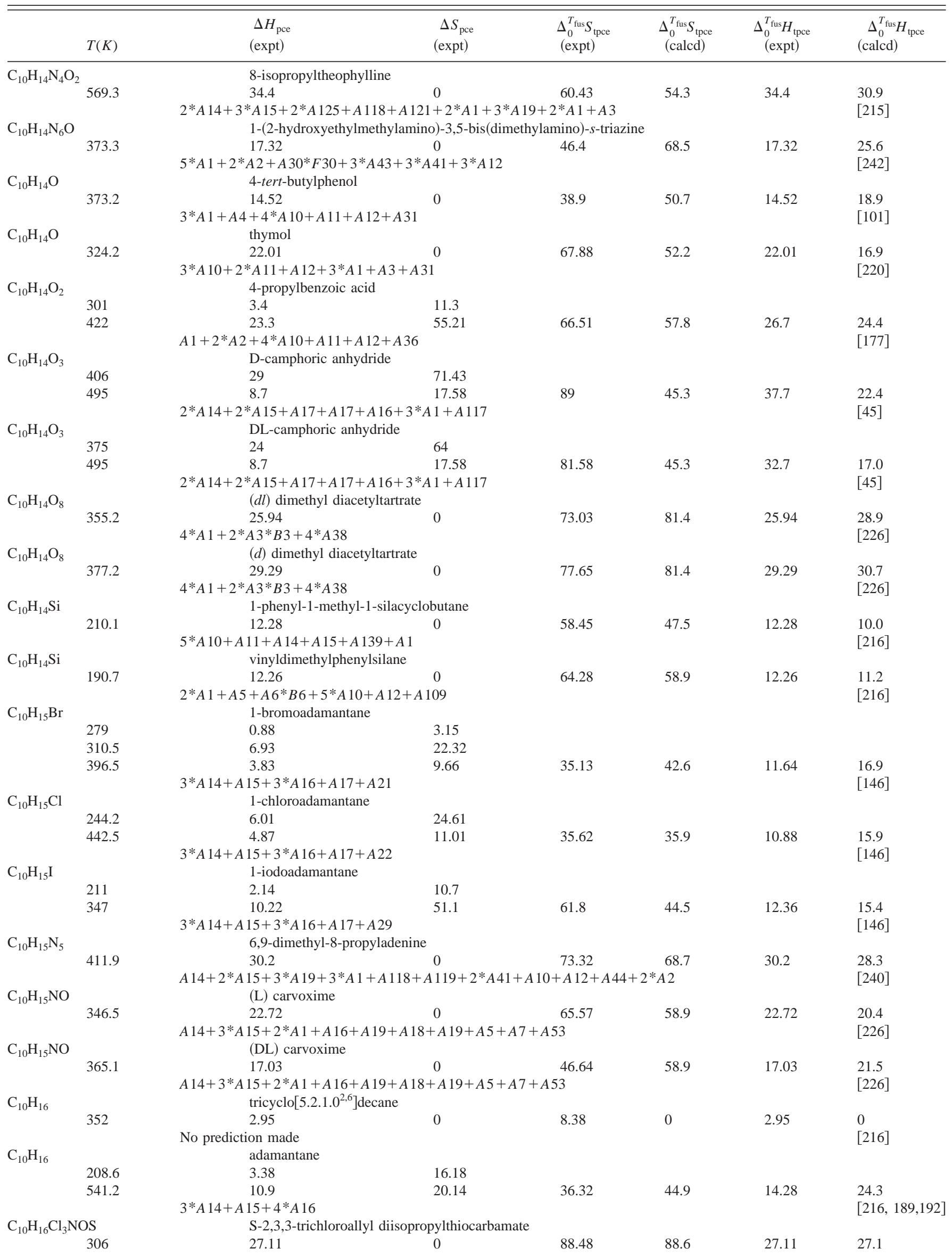


Table 5. Experimental and calculated total phase change enthalpy and entropy of database-Continued

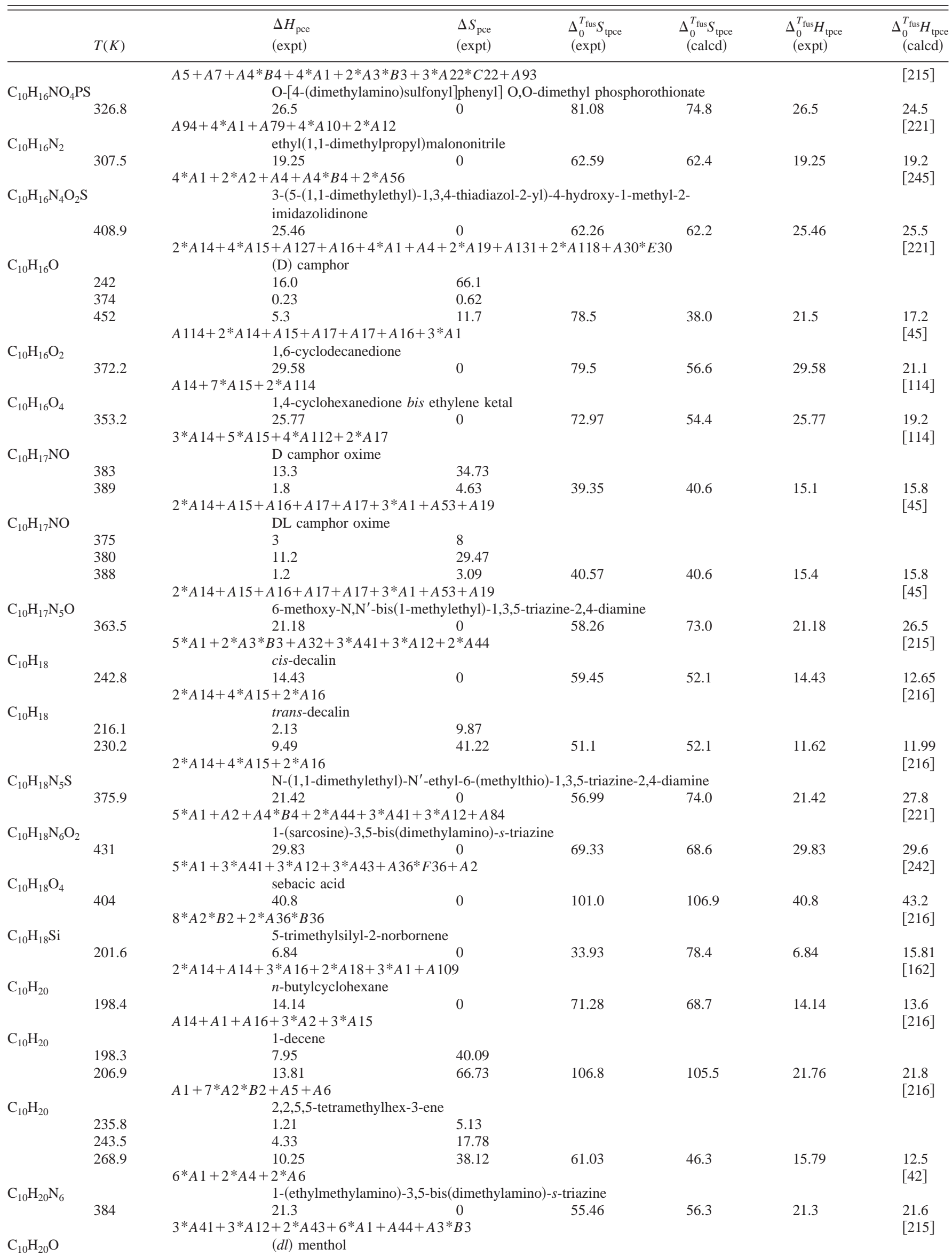


Table 5. Experimental and calculated total phase change enthalpy and entropy of database-Continued

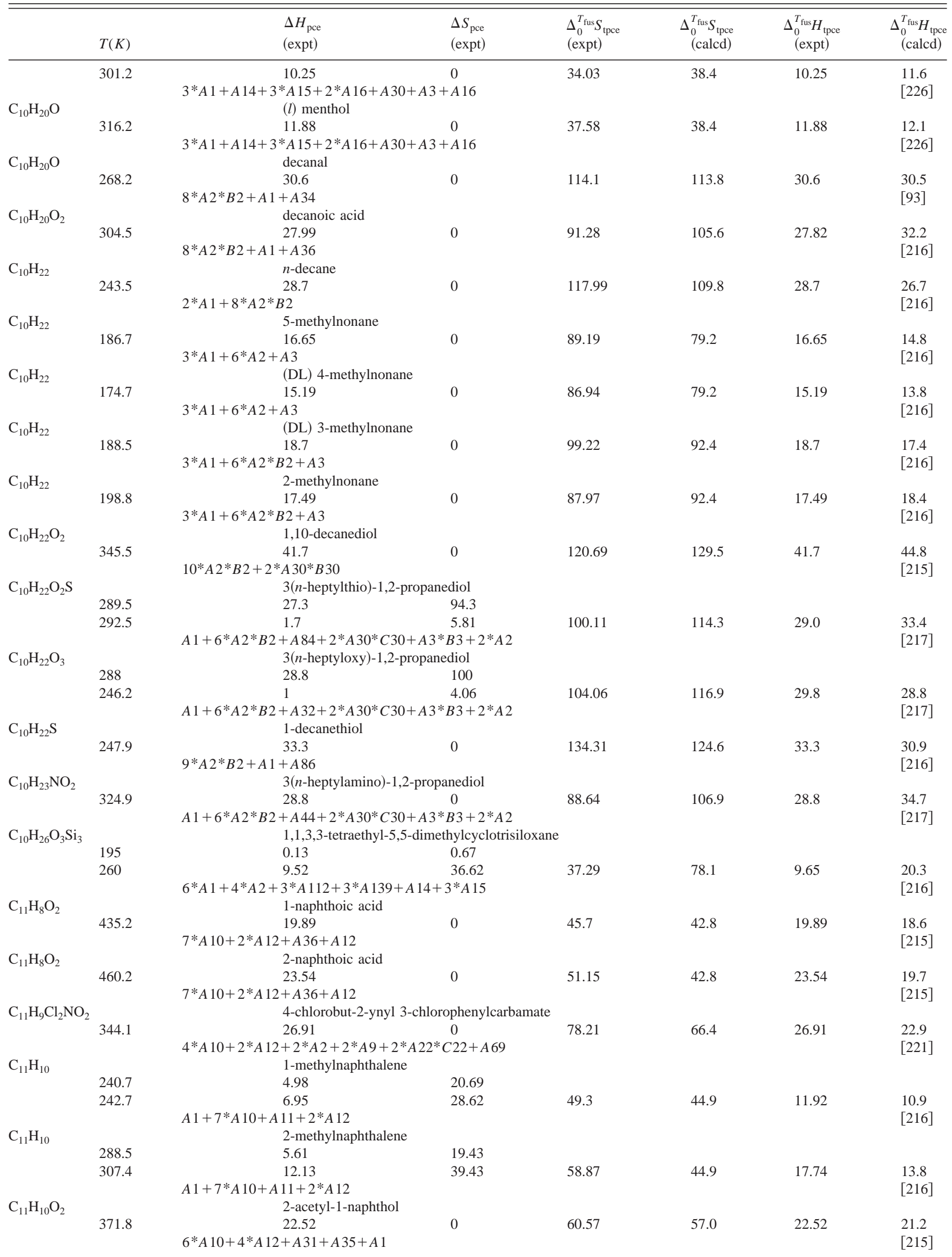


Table 5. Experimental and calculated total phase change enthalpy and entropy of database-Continued

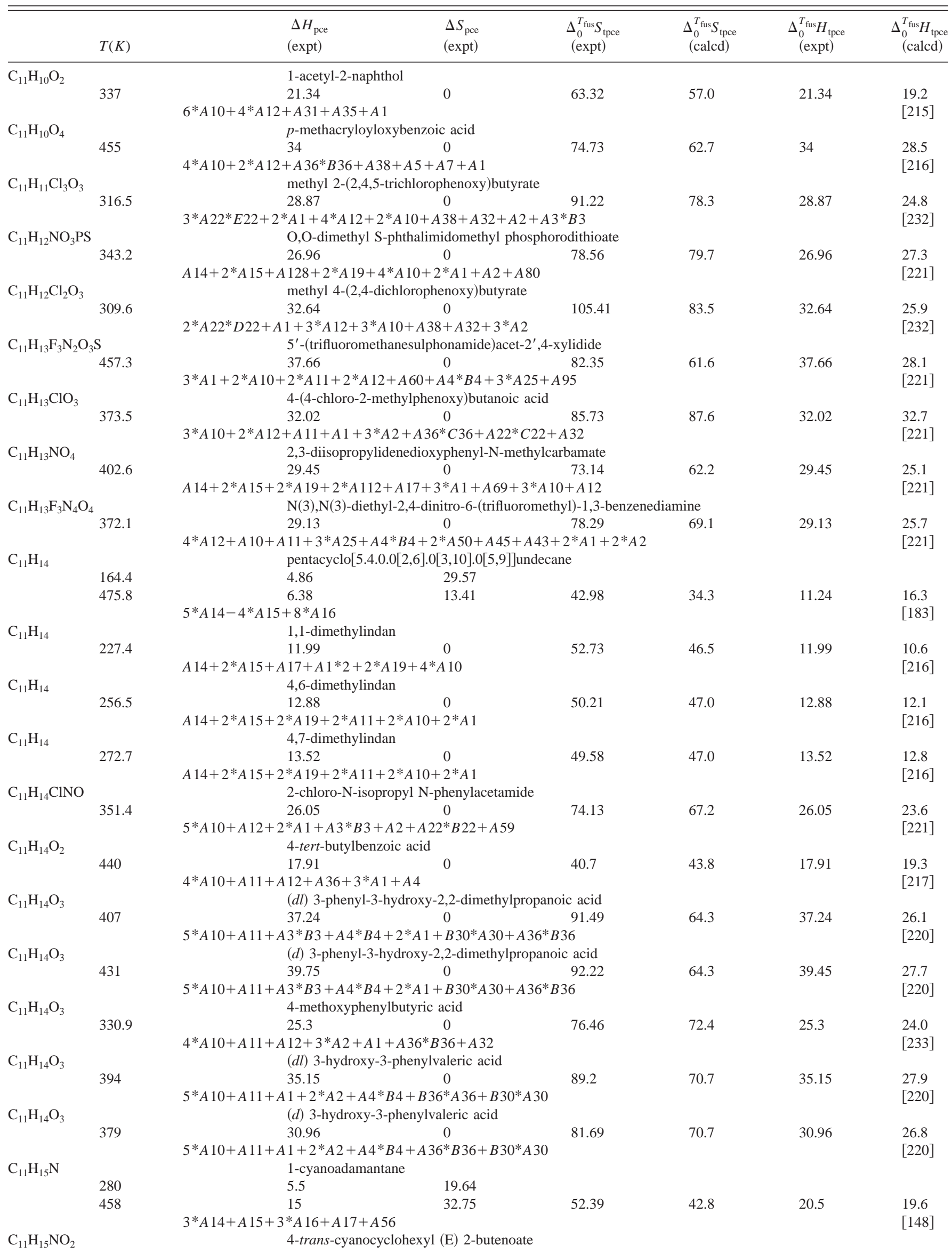


Table 5. Experimental and calculated total phase change enthalpy and entropy of database-Continued

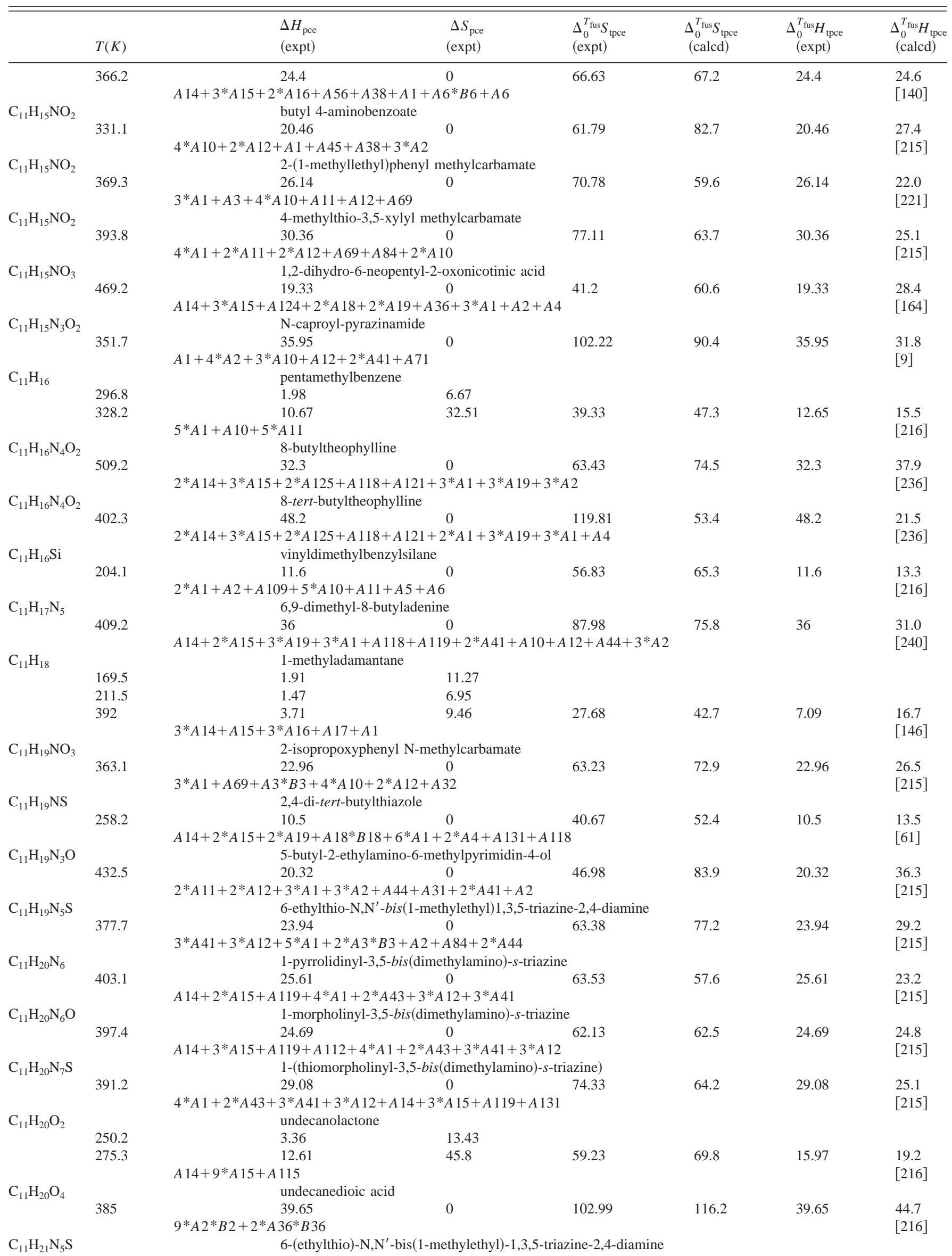


Table 5. Experimental and calculated total phase change enthalpy and entropy of database-Continued

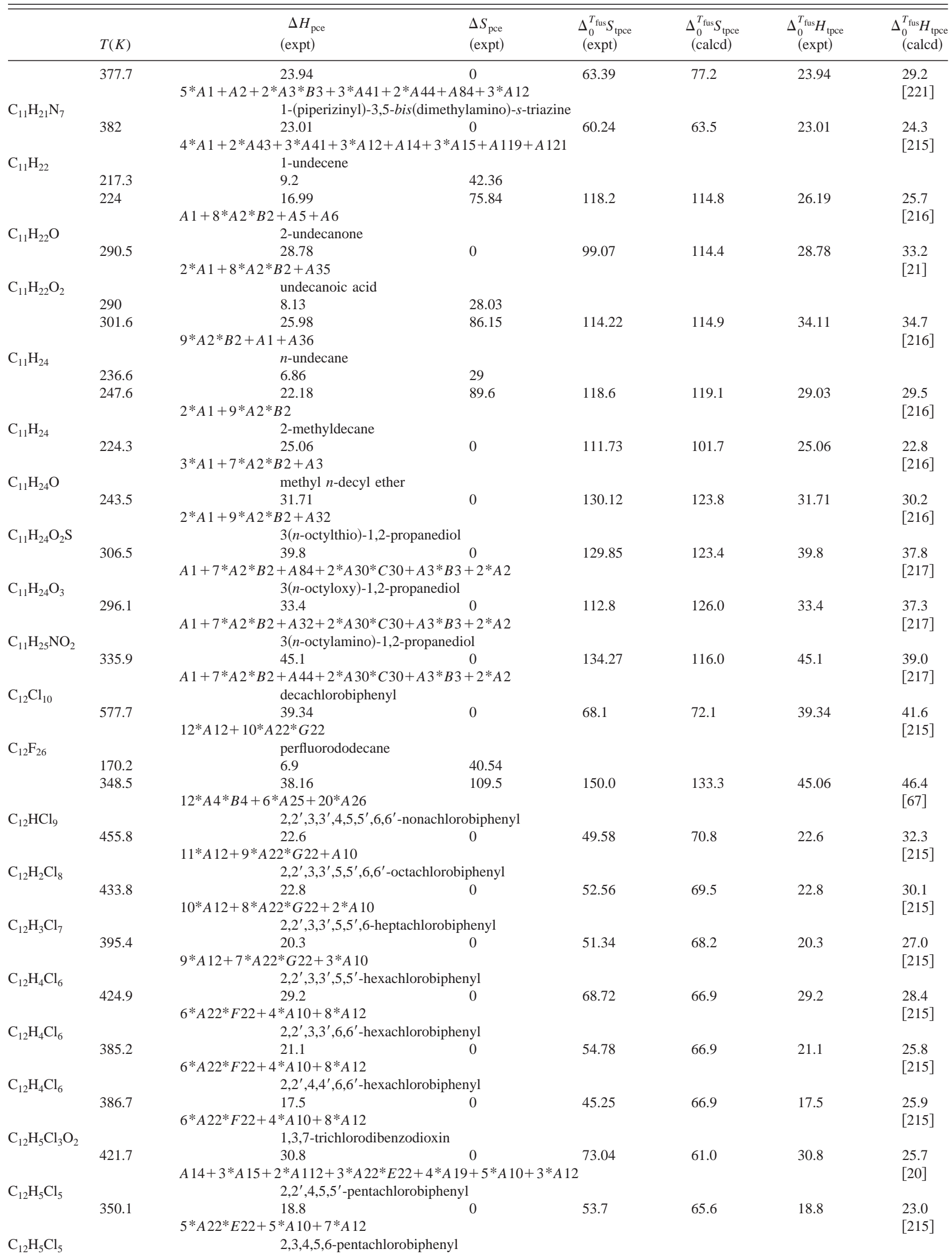


Table 5. Experimental and calculated total phase change enthalpy and entropy of database-Continued

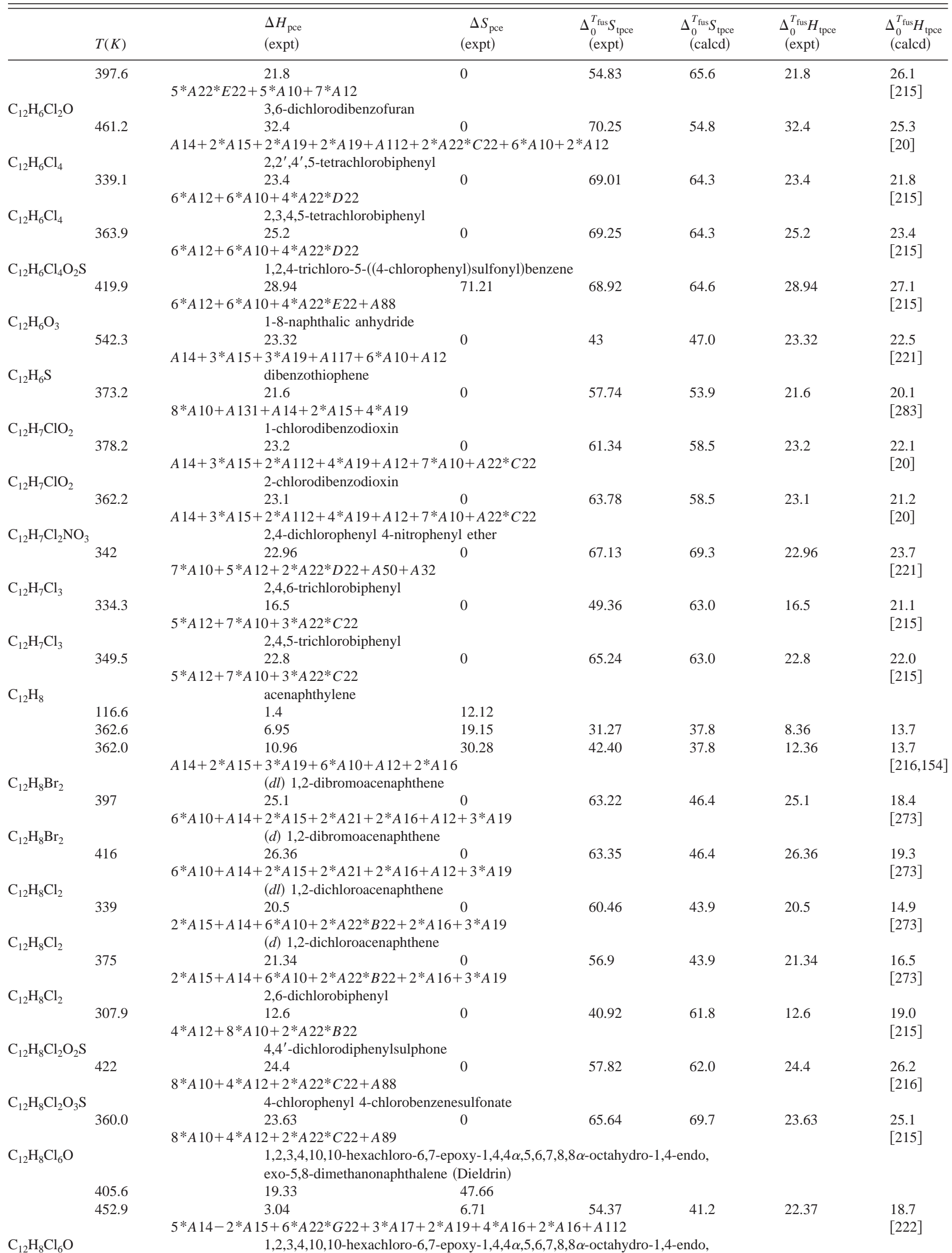


Table 5. Experimental and calculated total phase change enthalpy and entropy of database-Continued

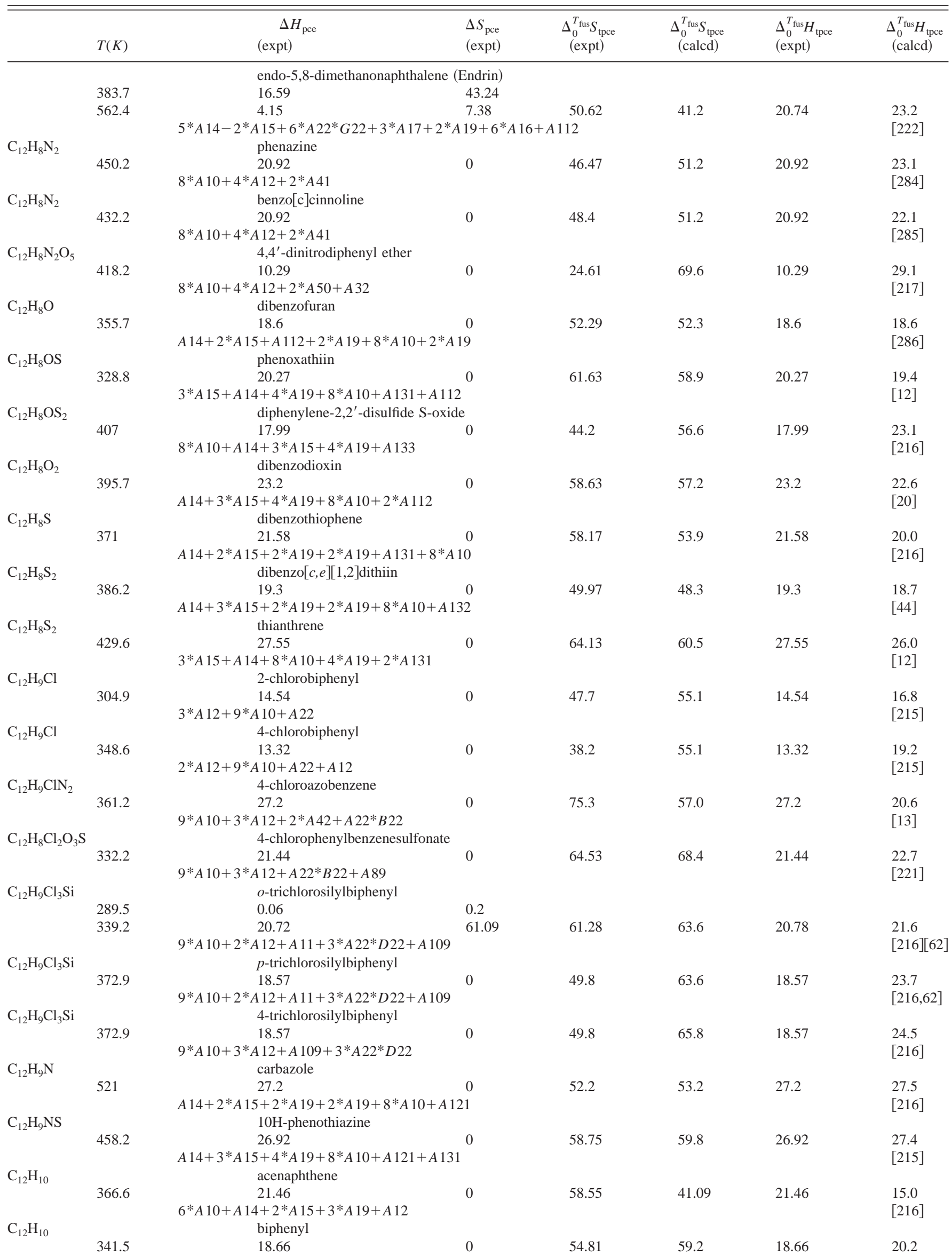


Table 5. Experimental and calculated total phase change enthalpy and entropy of database-Continued

\begin{tabular}{|c|c|c|c|c|c|c|c|}
\hline & $T(K)$ & $\begin{array}{r}\Delta H_{\text {pce }} \\
(\text { expt) }\end{array}$ & $\begin{array}{l}\Delta S_{\text {pce }} \\
(\text { expt) }\end{array}$ & $\begin{array}{l}\Delta_{0}^{T_{\text {fus }}} S_{\text {tpce }} \\
(\text { expt })\end{array}$ & $\begin{array}{c}\Delta_{0}^{T_{\text {fus }}} S_{\text {tpce }} \\
\quad \text { (calcd) }\end{array}$ & $\begin{array}{c}\Delta_{0}^{T_{\text {fus }}} H_{\text {tpce }} \\
\quad(\text { expt })\end{array}$ & $\begin{array}{c}\Delta_{0}^{T_{\text {fus }}} H_{\text {tpce }} \\
\quad \text { (calcd) }\end{array}$ \\
\hline & & $10 * A 10+2 * A 12$ & & & & & [216] \\
\hline \multirow{3}{*}{$\mathrm{C}_{12} \mathrm{H}_{10} \mathrm{~N}_{2}$} & & trans-azobenzene & & & & & \\
\hline & 341.1 & 22.53 & 0 & 66.06 & 55.7 & 22.53 & 19.0 \\
\hline & & $10 * A 10+2 * A 12+2 * A 42$ & & & & & {$[216]$} \\
\hline \multirow{3}{*}{$\mathrm{C}_{12} \mathrm{H}_{10} \mathrm{~N}_{2} \mathrm{O}$} & & azoxybenzene & & & & & \\
\hline & 309.2 & 17.93 & 0 & 57.99 & 64.3 & 17.93 & 19.9 \\
\hline & & $10 * A 10+2 * A 12+A 54+A 42$ & & & & & [215] \\
\hline \multirow[t]{3}{*}{$\mathrm{C}_{12} \mathrm{H}_{10} \mathrm{~N}_{2} \mathrm{O}$} & & 4-hydroxyazobenzene & & & & & \\
\hline & 425.2 & 32.99 & 0 & 77.59 & 61.0 & 32.99 & 26.0 \\
\hline & & $9 * A 10+3 * A 12+2 * A 42+A 31$ & & & & & {$[13]$} \\
\hline \multirow{3}{*}{$\mathrm{C}_{12} \mathrm{H}_{10} \mathrm{~N}_{4} \mathrm{O}_{2}$} & & 4-(4-nitrophenylazo)aniline & & & & & \\
\hline & 488.2 & 31.88 & 0 & 65.3 & 65.0 & 31.88 & 31.7 \\
\hline & & $8 * A 10+4 * A 12+2 * A 42+A 50+A 45$ & & & & & {$[13]$} \\
\hline \multirow{3}{*}{$\mathrm{C}_{12} \mathrm{H}_{10} \mathrm{O}$} & & $o$-hydroxybiphenyl & & & & & \\
\hline & 330.6 & 16.21 & 0 & 48.12 & 64.6 & 16.21 & 21.3 \\
\hline & & $9 * A 10+A 31+3 * A 12$ & & & & & {$[63]$} \\
\hline \multirow{3}{*}{$\mathrm{C}_{12} \mathrm{H}_{10} \mathrm{O}$} & & diphenyl ether & & & & & \\
\hline & 300 & 17.21 & 0 & 57.32 & 63.9 & 17.21 & 19.2 \\
\hline & & $10 * A 10+2 * A 12+A 32$ & & & & & [216] \\
\hline \multirow{3}{*}{$\mathrm{C}_{12} \mathrm{H}_{10} \mathrm{O}_{2}$} & & 1-naphthaleneacetic acid & & & & & \\
\hline & 405.3 & 22.26 & 0 & 54.92 & 47.8 & 22.26 & 19.4 \\
\hline & & $7 * A 10+2 * A 12+A 11+A 2+A 36$ & & & & & [215] \\
\hline \multirow{3}{*}{$\mathrm{C}_{12} \mathrm{H}_{10} \mathrm{O}_{2}$} & & 2-carbomethoxynaphthalen & & & & & \\
\hline & 350.2 & 27.1 & 0 & 77.4 & 54.7 & 27.1 & 19.2 \\
\hline & & $7 * A 10+A 1+A 38+3 * A 12$ & & & & & [247] \\
\hline \multirow[t]{3}{*}{$\mathrm{C}_{12} \mathrm{H}_{10} \mathrm{O}_{2}$} & & 1-acetoxynaphthalene & & & & & \\
\hline & 319.2 & 20.21 & 0 & 63.31 & 54.7 & 20.21 & 17.5 \\
\hline & & $7 * A 10+3 * A 12+A 1+A 38$ & & & & & [118] \\
\hline \multirow[t]{3}{*}{$\mathrm{C}_{12} \mathrm{H}_{10} \mathrm{O}_{2}$} & & 2-acetoxynaphthalene & & & & & \\
\hline & 342.2 & 20.05 & 0 & 58.59 & 54.7 & 20.05 & 18.7 \\
\hline & & $7 * A 10+3 * A 12+A 1+A 38$ & & & & & [118] \\
\hline \multirow[t]{3}{*}{$\mathrm{C}_{12} \mathrm{H}_{11} \mathrm{Cl}_{2} \mathrm{NO}$} & & 3,5-dichloro-N-(1,1-dimeth & yl-2-prop & zamide & & & \\
\hline & 428.4 & 28.68 & 0 & 66.94 & 58.1 & 28.68 & 24.9 \\
\hline & & $3 * A 12+3 * A 10+A 60+2 * A 22 * C 22+$ & $2 * A 1+A$ & $48+A 9$ & & & {$[221]$} \\
\hline \multirow[t]{3}{*}{$\mathrm{C}_{12} \mathrm{H}_{11} \mathrm{~N}$} & & diphenylamine & & & & & \\
\hline & 326.2 & 17.86 & 0 & 54.75 & 53.9 & 17.86 & 17.6 \\
\hline & & $10 * A 10+2 * A 12+A 44$ & & & & & [215] \\
\hline $\mathrm{C}_{12} \mathrm{H}_{11} \mathrm{~N}$ & & 2-aminobiphenyl & & & & & \\
\hline & 322.3 & 13.99 & 0 & 43.4 & 65.7 & 13.99 & 21.2 \\
\hline & & $9 * A 10+3 * A 12+A 45$ & & & & & [205] \\
\hline $\mathrm{C}_{12} \mathrm{H}_{11} \mathrm{NO}$ & & 1-naphthaleneacetamide & & & & & \\
\hline & 455.5 & 32.82 & 0 & 72.05 & 62.4 & 32.82 & 28.4 \\
\hline & & $7 * A 10+2 * A 12+A 11+A 2+A 61$ & & & & & [221] \\
\hline $\mathrm{C}_{12} \mathrm{H}_{11} \mathrm{NO}_{2}$ & & 1-naphthyl methylcarbama & & & & & \\
\hline & 416.3 & 24.51 & 0 & 58.88 & 57.6 & 24.51 & 24.0 \\
\hline & & $7 * A 10+3 * A 12+A 1+A 69$ & & & & & [221] \\
\hline $\mathrm{C}_{12} \mathrm{H}_{11} \mathrm{~N}_{3}$ & & $p$-phenylazoaniline & & & & & \\
\hline & 398.2 & 21.7 & 0 & 54.5 & 62.1 & 21.7 & 24.7 \\
\hline & & $9 * A 10+3 * A 12+2 * A 42+A 45$ & & & & & {$[13]$} \\
\hline $\mathrm{C}_{12} \mathrm{H}_{12}$ & & 1,8-dimethylnaphthalene & & & & & \\
\hline & 336.3 & 15.77 & 0 & 46.9 & 45.5 & 15.77 & 15.3 \\
\hline & & $2 * A 1+2 * A 11+6 * A 10+2 * A 12$ & & & & & [215] \\
\hline $\mathrm{C}_{12} \mathrm{H}_{12}$ & & 2,6-dimethylnaphthalene & & & & & \\
\hline & 383.3 & 25.06 & 0 & 65.39 & 45.5 & 25.06 & 17.4 \\
\hline & & $2 * A 1+2 * A 11+6 * A 10+2 * A 12$ & & & & & {$[215]$} \\
\hline $\mathrm{C}_{12} \mathrm{H}_{12}$ & & 2,7-dimethylnaphthalene & & & & & \\
\hline & 368.8 & 23.35 & 0 & 63.3 & 45.5 & 23.35 & 16.8 \\
\hline & & $2 * A 1+2 * A 11+6 * A 10+2 * A 12$ & & & & & {$[215]$} \\
\hline $\mathrm{C}_{12} \mathrm{H}_{12}$ & & 1,4-dimethylnaphthalene & & & & & \\
\hline & 279.9 & 10.6 & 0 & 37.87 & 45.5 & 10.6 & 12.7 \\
\hline & & $2 * A 1+2 * A 11+2 * A 12+6 * A 10$ & & & & & {$[215]$} \\
\hline $\mathrm{C}_{12} \mathrm{H}_{12}$ & & 2,3-dimethylnaphthalene & & & & & \\
\hline & 378 & 15.9 & 0 & 42.06 & 45.5 & 15.9 & 17.2 \\
\hline $\mathrm{C}_{1} \mathrm{H}_{1} \mathrm{Ge}$ & & $2 * A 1+2 * A 11+2 * A 12+6 * A 10$ & & & & & [215] \\
\hline $\mathrm{C}_{12 \mathrm{11} 12}$ & 240.2 & 11.91 & 0 & 49.58 & 44.5 & 11.91 & 10.7 \\
\hline
\end{tabular}


Table 5. Experimental and calculated total phase change enthalpy and entropy of database-Continued

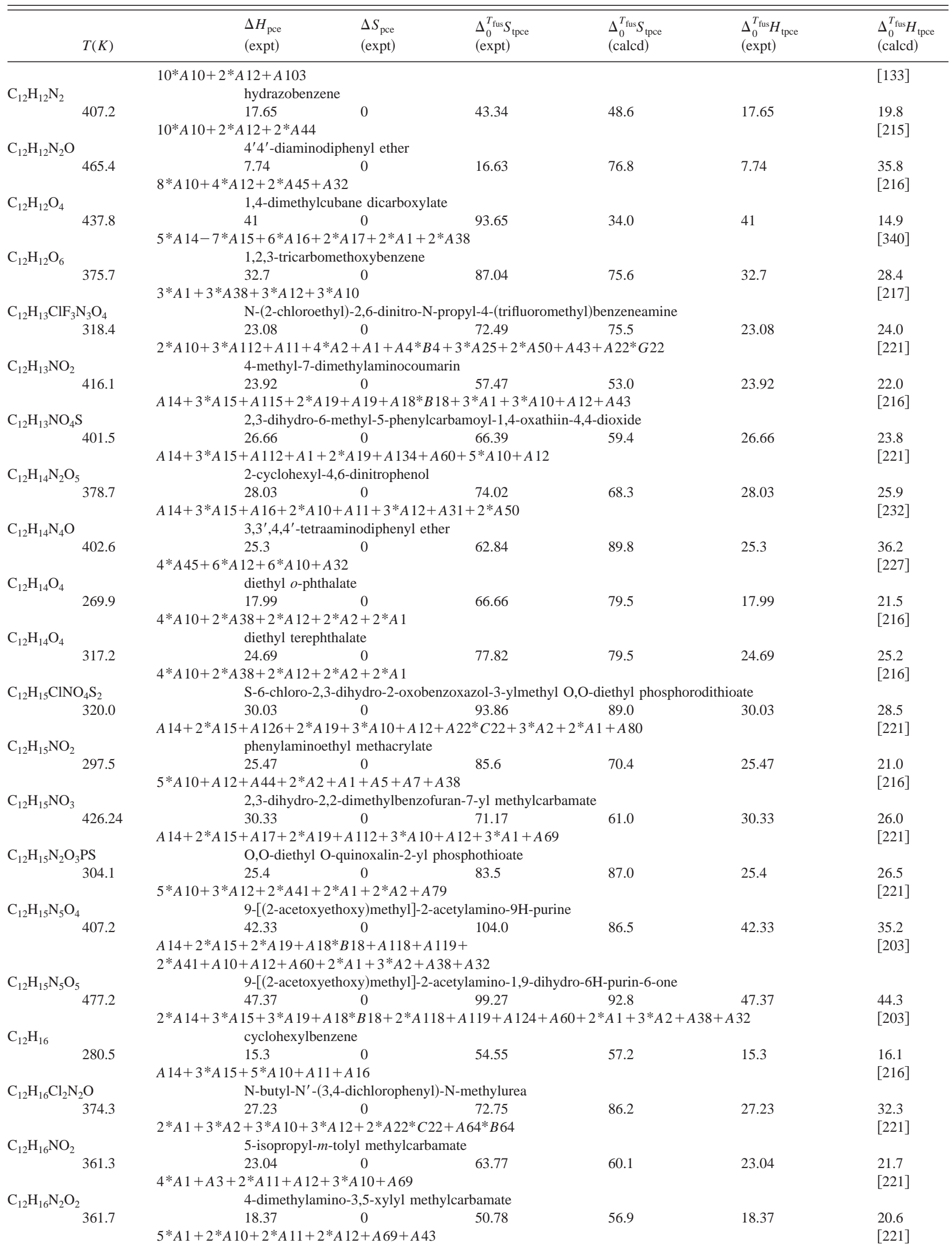


Table 5. Experimental and calculated total phase change enthalpy and entropy of database-Continued

\begin{tabular}{|c|c|c|c|c|c|c|c|}
\hline & $T(K)$ & $\begin{array}{r}\Delta H_{\mathrm{pce}} \\
(\text { expt })\end{array}$ & $\begin{array}{l}\Delta S_{\mathrm{pce}} \\
(\operatorname{expt})\end{array}$ & $\begin{array}{l}\Delta_{0}^{T_{\text {fus }}} S_{\text {tpce }} \\
(\operatorname{expt})\end{array}$ & $\begin{array}{l}\Delta_{0}^{T_{\text {fus }}} S_{\text {tpce }} \\
\quad(\text { calcd })\end{array}$ & $\begin{array}{l}\Delta_{0}^{T_{\text {fus }}} H_{\text {tpce }} \\
\quad(\operatorname{expt})\end{array}$ & $\begin{array}{l}\Delta_{0}^{T_{\text {fus }}} H_{\text {tpce }} \\
\quad \text { (calcd) }\end{array}$ \\
\hline \multirow[t]{3}{*}{$\mathrm{C}_{12} \mathrm{H}_{16} \mathrm{~N}_{3} \mathrm{O}_{6} \mathrm{~S}$} & & 4-meth & ,N-dipro & & & & \\
\hline & 424.3 & 28.05 & 0 & 66.1 & 79.7 & 28.05 & 33.8 \\
\hline & & \multicolumn{5}{|c|}{$3 * A 1+4 * A 2+4 * A 12+2 * A 10+A 43+2 * A 50+A 88$} & {$[221]$} \\
\hline \multicolumn{2}{|l|}{$\mathrm{C}_{12} \mathrm{H}_{16} \mathrm{~N}_{3} \mathrm{O}_{3} \mathrm{PS}_{2}$} & \multicolumn{5}{|c|}{$\begin{array}{l}\text { S-(3,4-dihydro-4-oxobenzo[d]-[1,2,3]-triazin-3-ylmethyl) O,O-diethyl } \\
\text { phoshorodithioate }\end{array}$} & \\
\hline & 322.2 & 25.22 & 0 & 78.26 & 75.9 & 25.22 & 24.4 \\
\hline \multirow[t]{3}{*}{$\mathrm{C}_{12} \mathrm{H}_{17} \mathrm{NO}_{2}$} & & \multicolumn{5}{|c|}{$\begin{array}{c}A 14+3 * A 15+2 * A 118+2 * A 19+4 * A 10+A 125+3 * A 2+2 * A 1+A 80 \\
\text { pentyl 4-aminobenzoate }\end{array}$} & {$[221]$} \\
\hline & 325.1 & 23.93 & 0 & 73.61 & 89.9 & 23.93 & 29.2 \\
\hline & & $4 * A 10+2 * A 12+A 1$ & & & & & {$[215]$} \\
\hline \multirow[t]{4}{*}{$\mathrm{C}_{12} \mathrm{H}_{18}$} & & hexame & & & & & \\
\hline & 383.7 & 1.76 & 4.58 & & & & \\
\hline & 438.7 & 20.63 & 47.02 & 51.6 & 47.9 & 22.38 & 21.0 \\
\hline & & $6^{*} A 1+6^{*} A 11$ & & & & & {$[216]$} \\
\hline \multirow{3}{*}{$\mathrm{C}_{12} \mathrm{H}_{18} \mathrm{~N}_{2} \mathrm{O}$} & & N,N-di & hyl)pher & & & & \\
\hline & 430.5 & 33.87 & 0 & 78.68 & 66.7 & 33.87 & 28.7 \\
\hline & & $4 * A 1+A 3+4 * A 10$ & & & & & {$[215]$} \\
\hline \multirow{3}{*}{$\mathrm{C}_{12} \mathrm{H}_{18} \mathrm{~N}_{2} \mathrm{O}_{2}$} & & 3,5-dim & phenyl n & amate & & & \\
\hline & 361.7 & 18.37 & 0 & 50.79 & 56.9 & 18.37 & 20.6 \\
\hline & & $5 * A 1+2 * A 10+2 *$ & & & & & {$[215]$} \\
\hline \multirow[t]{3}{*}{$\mathrm{C}_{12} \mathrm{H}_{18} \mathrm{~N}_{4} \mathrm{O}_{2}$} & & 8-penty & & & & & \\
\hline & 498.4 & 35.1 & 0 & 70.43 & 81.6 & 35.1 & 40.7 \\
\hline & & $2 * A 14+3 * A 15+2 *$ & $A 1+3$ & $A 2$ & & & {$[215]$} \\
\hline \multirow[t]{3}{*}{$\mathrm{C}_{12} \mathrm{H}_{18} \mathrm{~N}_{4} \mathrm{O}_{6} \mathrm{~S}$} & & 4-(N,N & robenze & amide & & & \\
\hline & 414.8 & 38.48 & 0 & 92.78 & 90.2 & 38.48 & 37.4 \\
\hline & & $2 * A 1+4 * A 2+2 * A$ & $43+A 96$ & & & & {$[221]$} \\
\hline \multirow{3}{*}{$\mathrm{C}_{12} \mathrm{H}_{18} \mathrm{O}_{2}$} & & 4-hexy & & & & & \\
\hline & 341.5 & 19.04 & 0 & 55.75 & 91.5 & 19.04 & 31.2 \\
\hline & & $3 * A 10+A 11+2 * A 1$ & & & & & {$[215]$} \\
\hline $\mathrm{C}_{12} \mathrm{H}_{19} \mathrm{ClNO}_{3}$ & & N-meth & 4-tert-bu & phosphor & & & \\
\hline & 332.0 & 21.98 & 0 & 66.19 & 66.2 & 21.98 & 22.0 \\
\hline & & $5 * A 1+A 4+2 * A 12$ & $22 * B 22$ & & & & {$[221]$} \\
\hline $\mathrm{C}_{12} \mathrm{H}_{20}$ & & 1,3-dim & & & & & \\
\hline & 221 & 7.36 & 33.3 & & & & \\
\hline & 245 & 0.92 & 3.76 & 37.06 & 40.4 & 8.28 & 9.9 \\
\hline & & $3 * A 14+A 15+2 * A 1$ & & & & & {$[146]$} \\
\hline $\mathrm{C}_{12} \mathrm{H}_{20} \mathrm{~N}_{4} \mathrm{O}_{2}$ & & 3-cyclo & -1-meth & iazine-2,4( & )-dione & & \\
\hline & 389.6 & 20.36 & 0 & 52.26 & 49.5 & 20.36 & 19.3 \\
\hline & & $2 * A 14+6^{*} A 15+2 *$ & $A 43+A$ & & & & {$[221]$} \\
\hline $\mathrm{C}_{12} \mathrm{H}_{20} \mathrm{O}_{2}$ & & 1,7-cyc & & & & & \\
\hline & 405.2 & 15.77 & 0 & 38.93 & 64.0 & 15.77 & 25.9 \\
\hline & & $A 14+9 * A 15+2 * A 1$ & & & & & [114] \\
\hline $\mathrm{C}_{12} \mathrm{H}_{20} \mathrm{O}_{3}$ & & $3,3,6,6$ & nhydride & & & & \\
\hline & 344.2 & 18.83 & 0 & 54.7 & 59.1 & 18.83 & 20.33 \\
\hline & & $A 14+6^{*} A 15+4^{*} A 1$ & & & & & {$[109]$} \\
\hline $\mathrm{C}_{12} \mathrm{H}_{20} \mathrm{O}_{4}$ & & 1,5 -сус & he ketal & & & & \\
\hline & 296.2 & 18.03 & 0 & 60.88 & 61.8 & 18.03 & 18.3 \\
\hline & & $3 * A 14+7 * A 15+4 *$ & & & & & {$[114]$} \\
\hline $\mathrm{C}_{12} \mathrm{H}_{22}$ & & bicyclo & & & & & \\
\hline & 256.1 & 1.54 & 6.01 & & & & \\
\hline & 267.5 & 0.74 & 2.77 & & & & \\
\hline & 273.5 & 7.08 & 25.89 & & & & \\
\hline & 277.2 & 6.78 & 24.46 & 59.13 & 59.5 & 15.4 & 16.5 \\
\hline & & $2 * A 14+6 * A 15+2 *$ & & & & & {$[216]$} \\
\hline $\mathrm{C}_{12} \mathrm{H}_{22} \mathrm{~N}_{2} \mathrm{O}_{2}$ & & 1,8-dia & ane & & & & \\
\hline & 517.4 & 13.6 & 26.29 & & & & \\
\hline & 617.8 & 49.3 & 79.8 & 106.1 & 79.5 & 62.9 & 49.1 \\
\hline & & $A 14+11 * A 15+2 * A$ & & & & & {$[248]$} \\
\hline $\mathrm{C}_{12} \mathrm{H}_{22} \mathrm{~N}_{6}$ & & 1-(pipe & no) $-s$-tri & & & & \\
\hline & 361.5 & 23.22 & 0 & 64.23 & 61.3 & 23.22 & 22.2 \\
\hline & & $A 14+3 * A 15+A 119$ & $2+3 * A$ & & & & {$[215]$} \\
\hline $\mathrm{C}_{12} \mathrm{H}_{22} \mathrm{O}_{2}$ & & octyl m & & & & & \\
\hline & 230.3 & 24.9 & 0 & 104.6 & 114.8 & 24.9 & 26.4 \\
\hline & & $2 * A 1+A 7+A 5+A 3$ & & & & & {$[227]$} \\
\hline $\mathrm{C}_{12} \mathrm{H}_{22} \mathrm{O}_{2}$ & & nonyl a & & & & & \\
\hline & 236.5 & 23.36 & 0 & 98.78 & 121.2 & 23.36 & 28.7 \\
\hline & & $A 1+A 6 * B 6+A 5+$ & & & & & {$[216]$} \\
\hline
\end{tabular}


Table 5. Experimental and calculated total phase change enthalpy and entropy of database-Continued

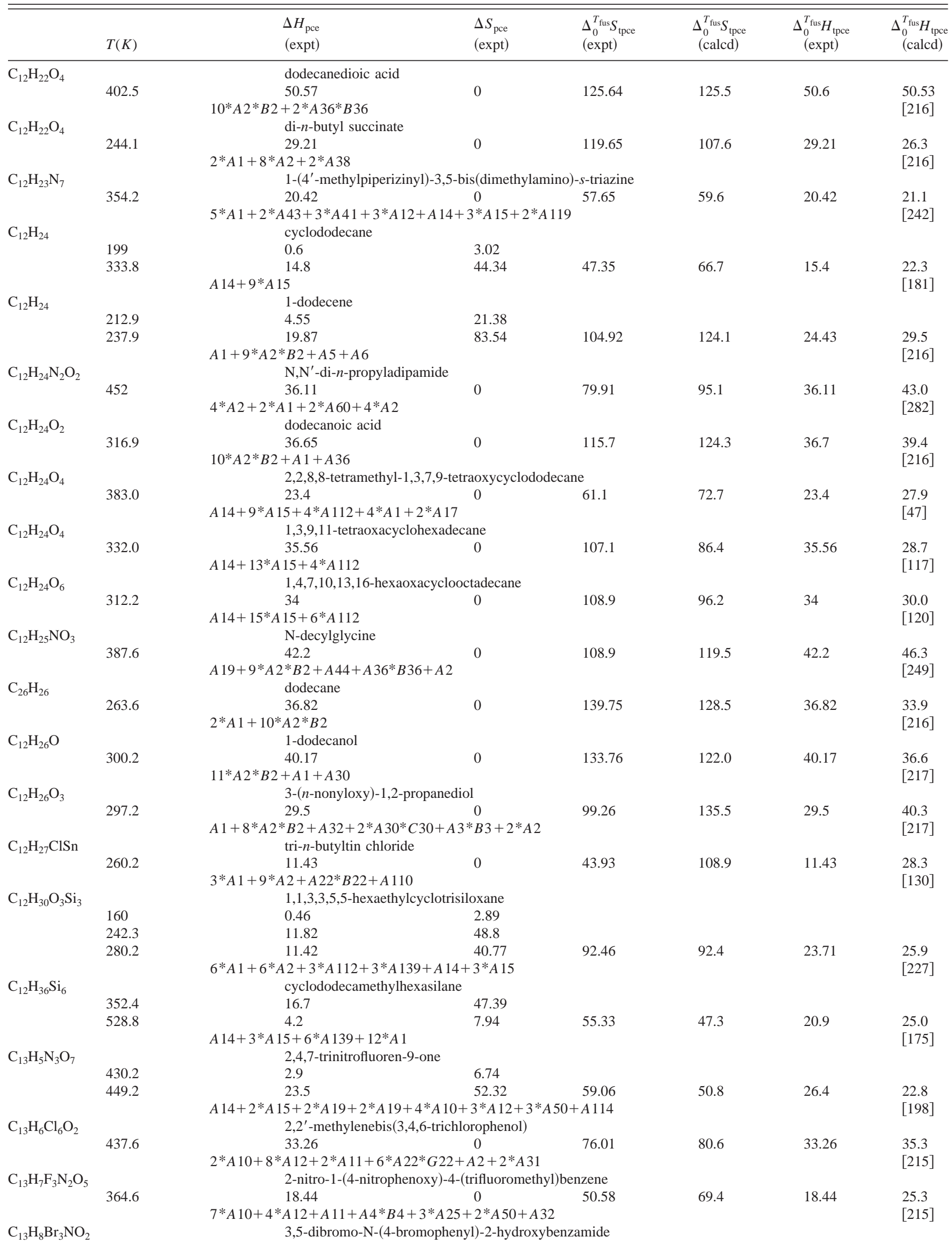


Table 5. Experimental and calculated total phase change enthalpy and entropy of database-Continued

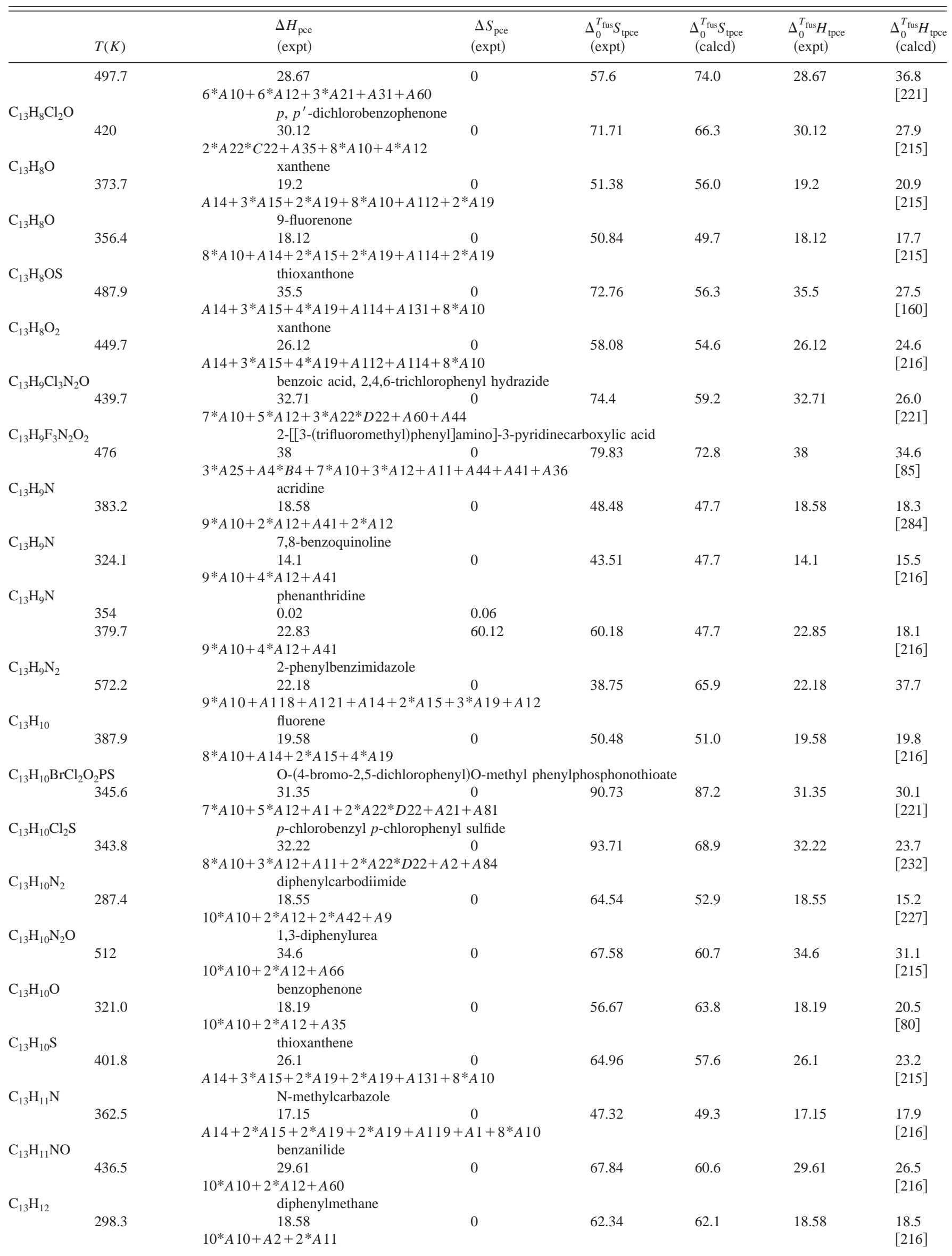


Table 5. Experimental and calculated total phase change enthalpy and entropy of database-Continued

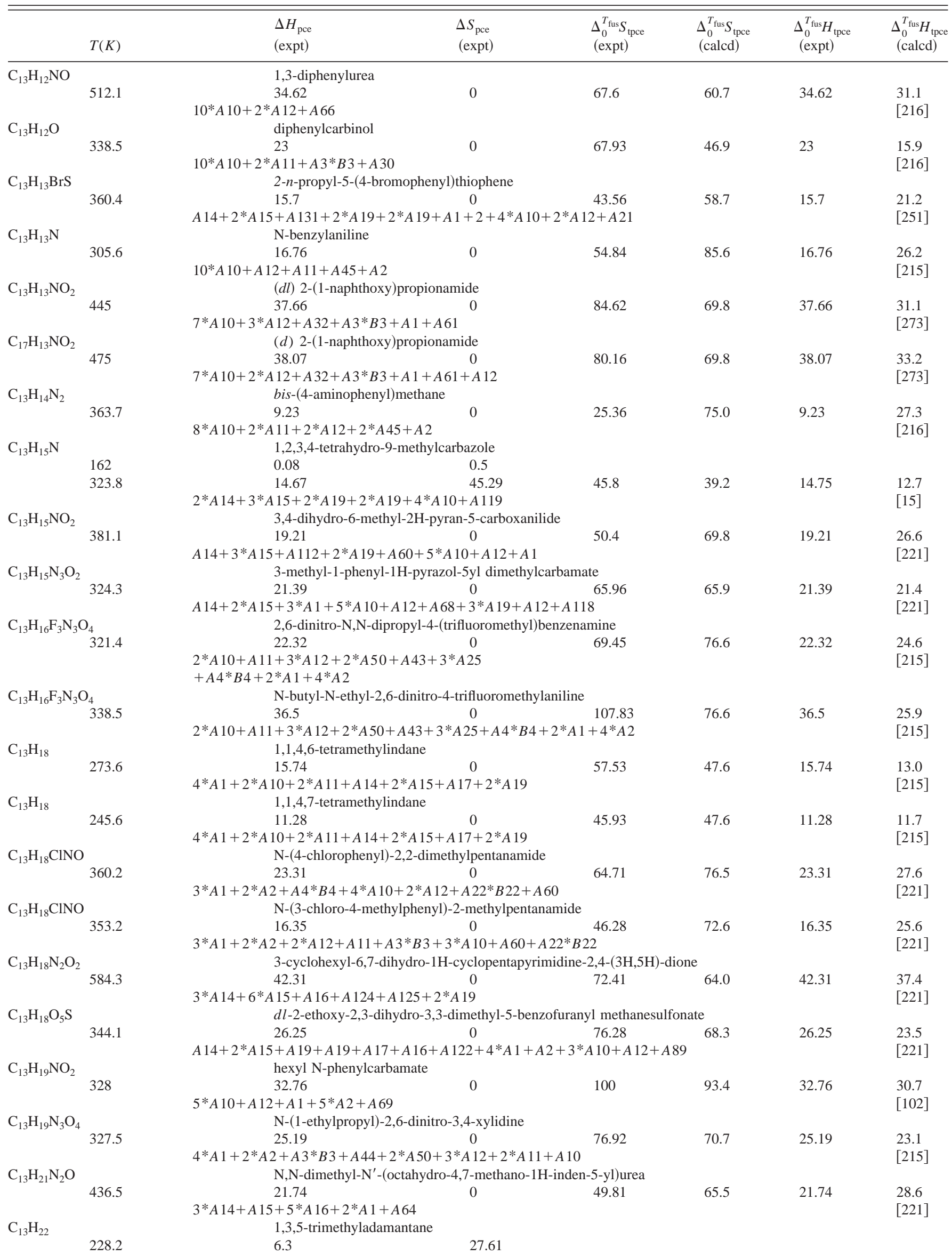


Table 5. Experimental and calculated total phase change enthalpy and entropy of database-Continued

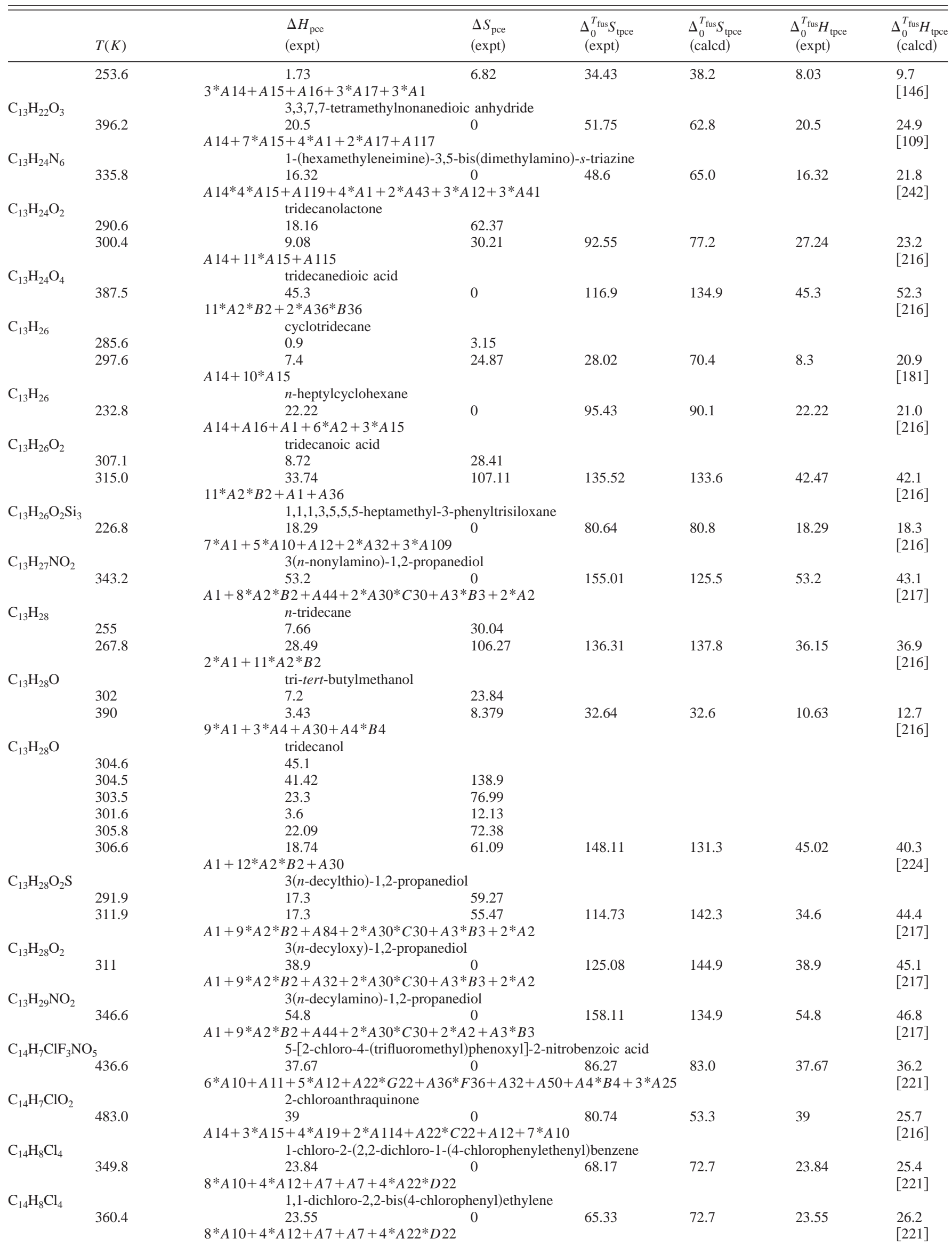


Table 5. Experimental and calculated total phase change enthalpy and entropy of database-Continued

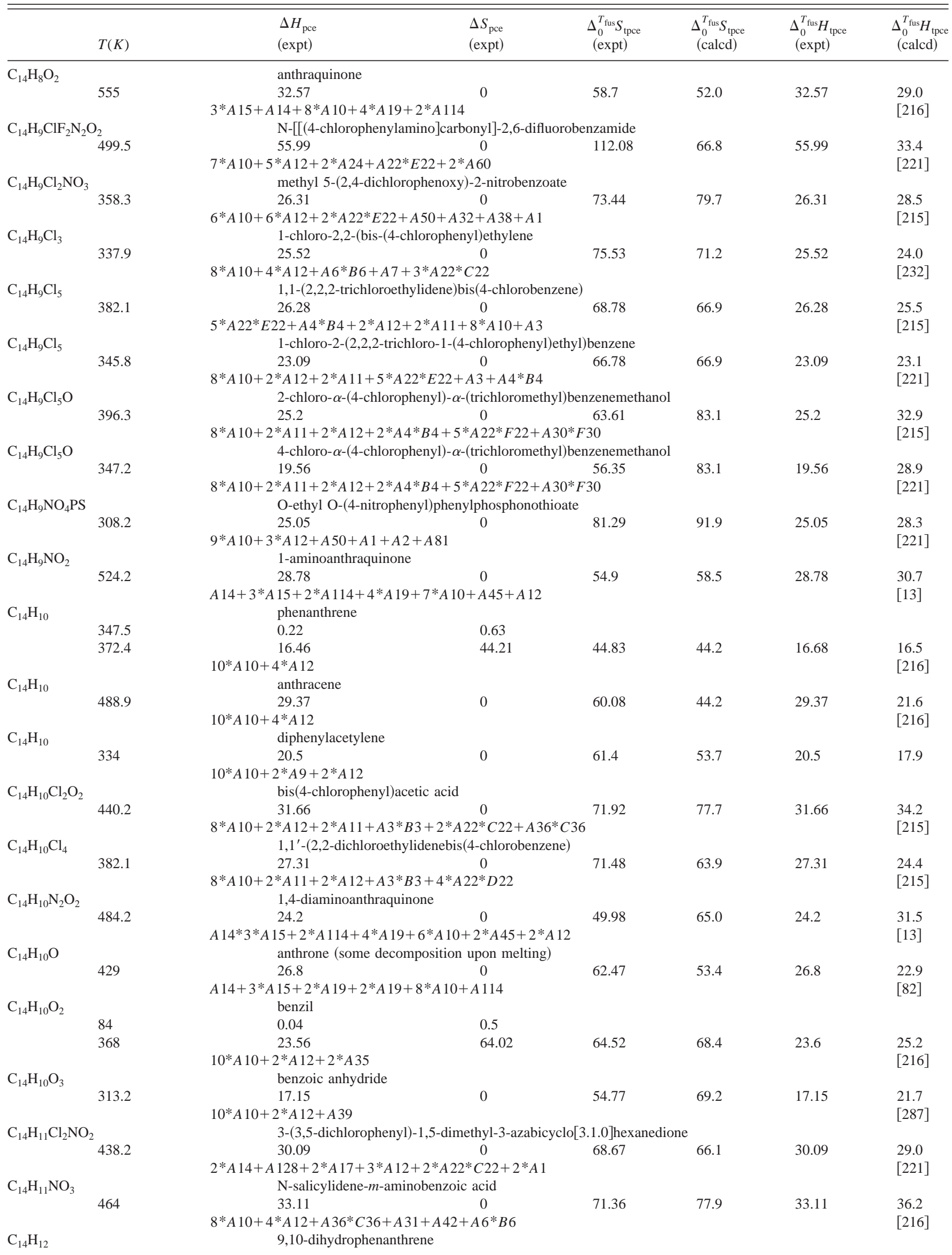


Table 5. Experimental and calculated total phase change enthalpy and entropy of database-Continued

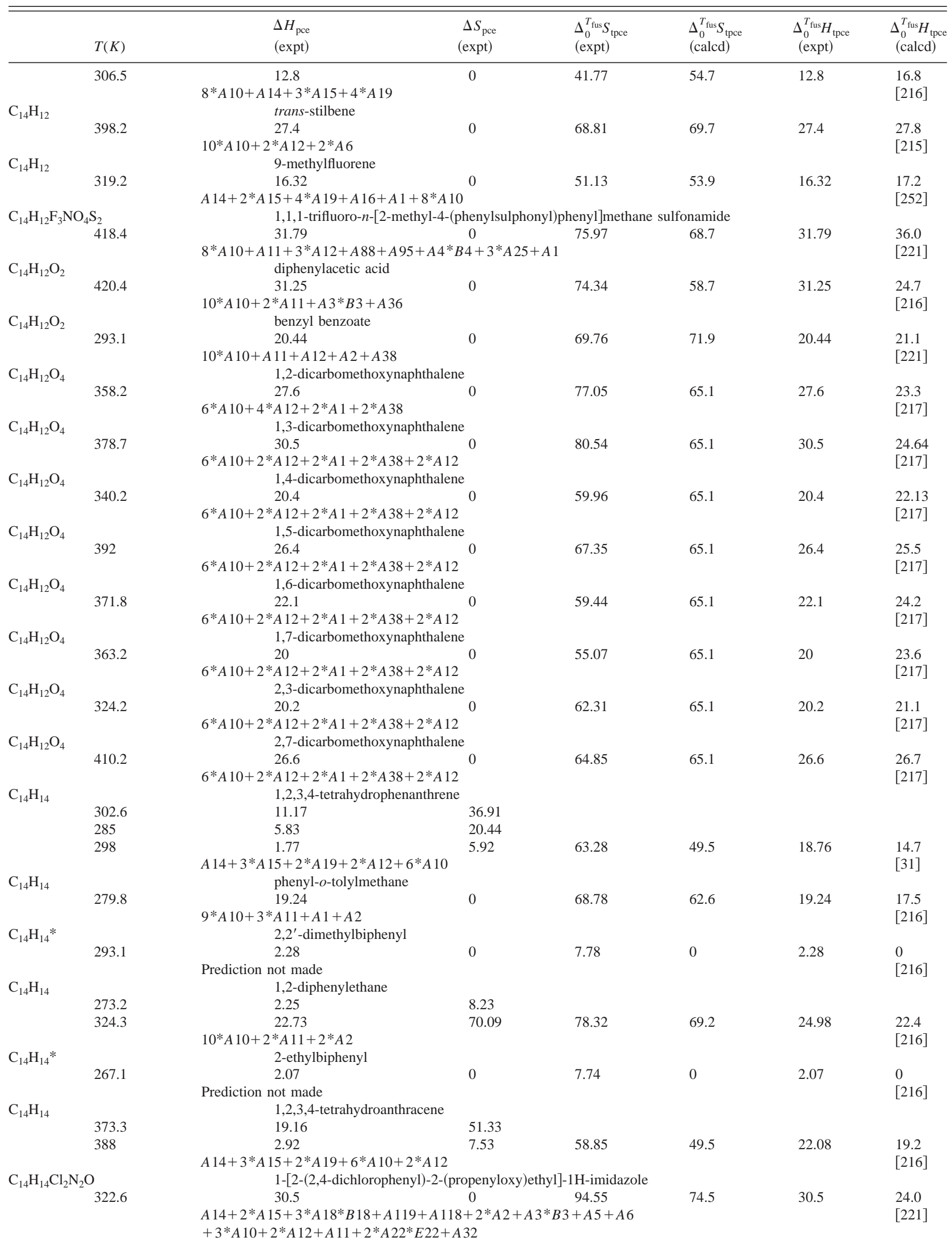


Table 5. Experimental and calculated total phase change enthalpy and entropy of database-Continued

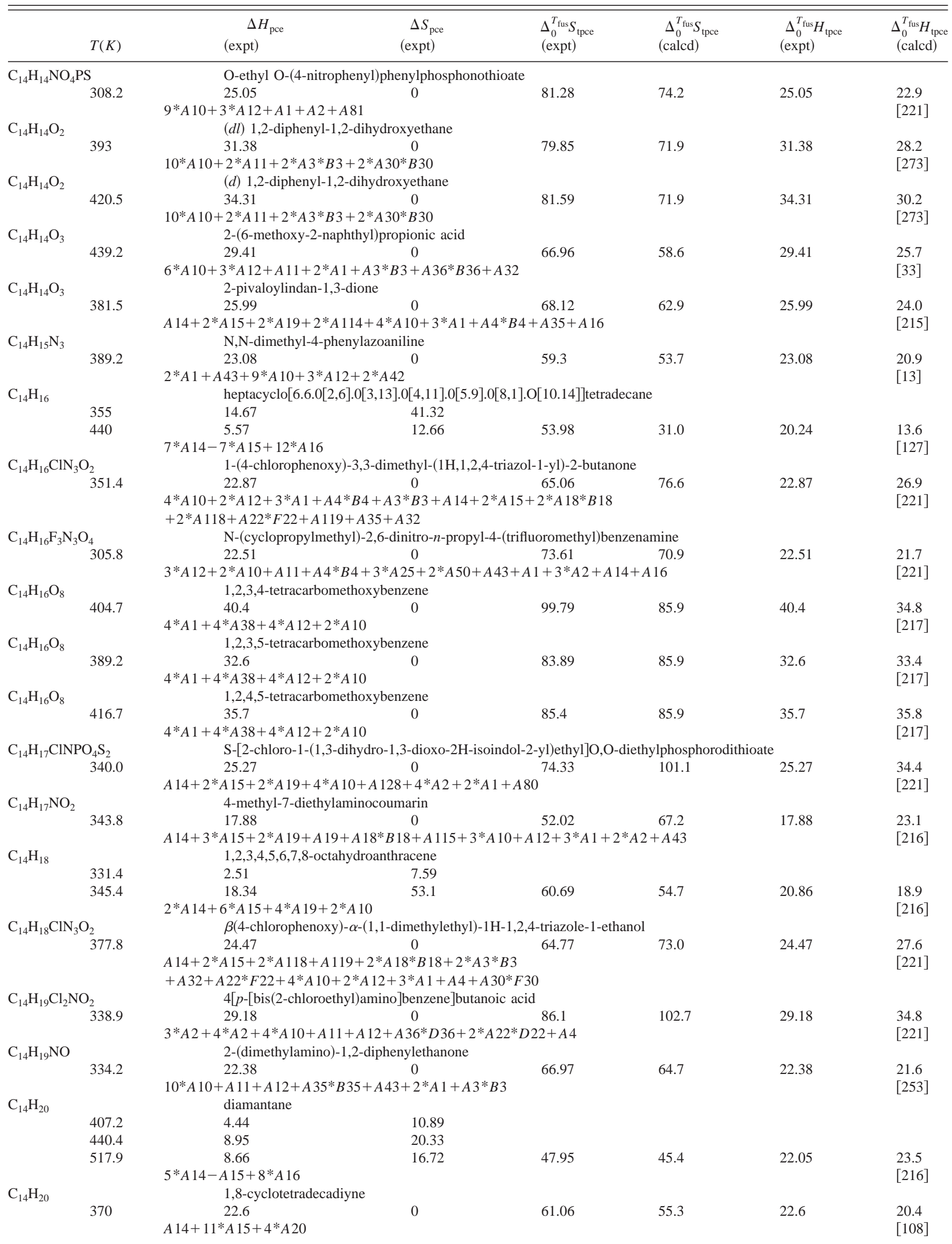


Table 5. Experimental and calculated total phase change enthalpy and entropy of database-Continued

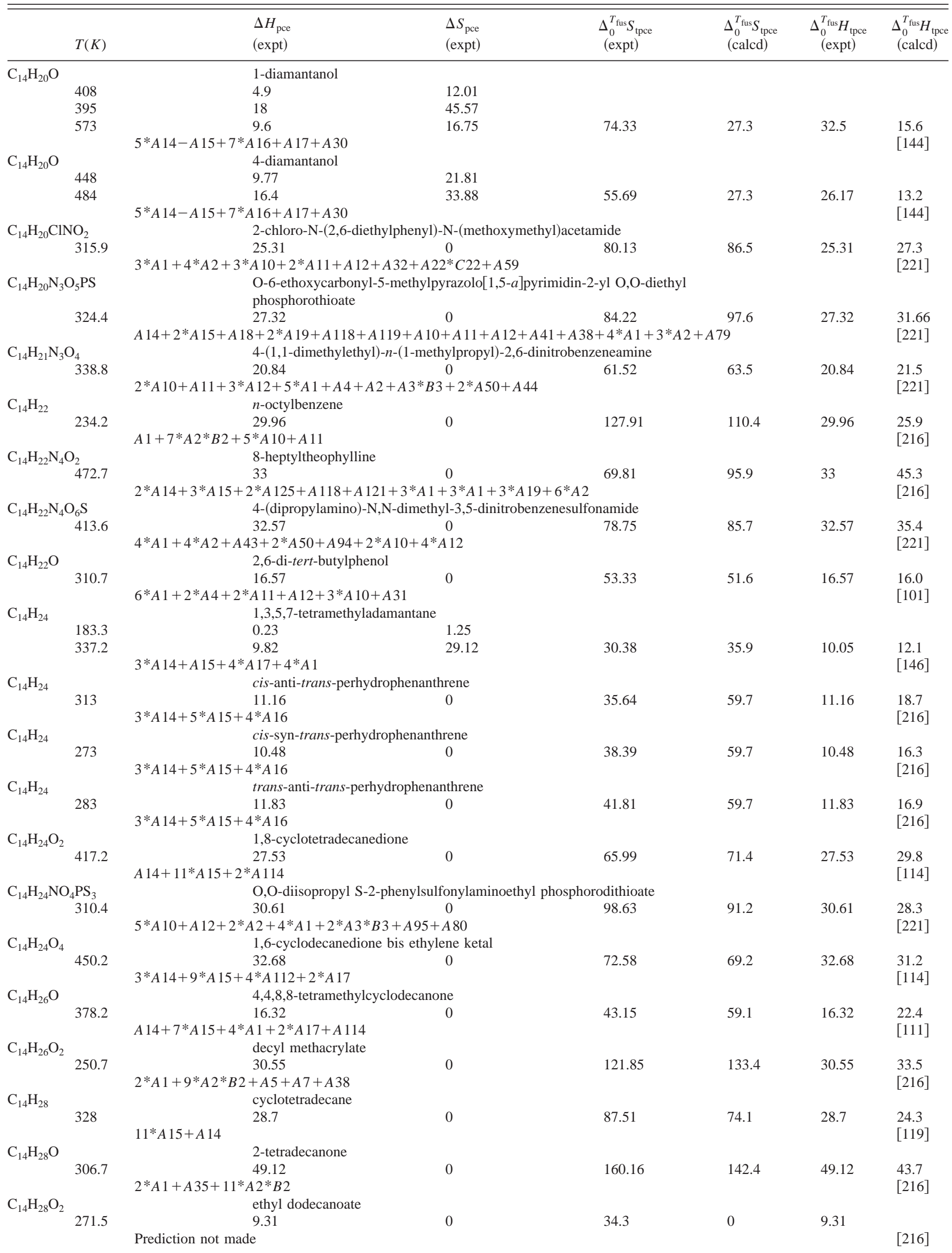


Table 5. Experimental and calculated total phase change enthalpy and entropy of database-Continued

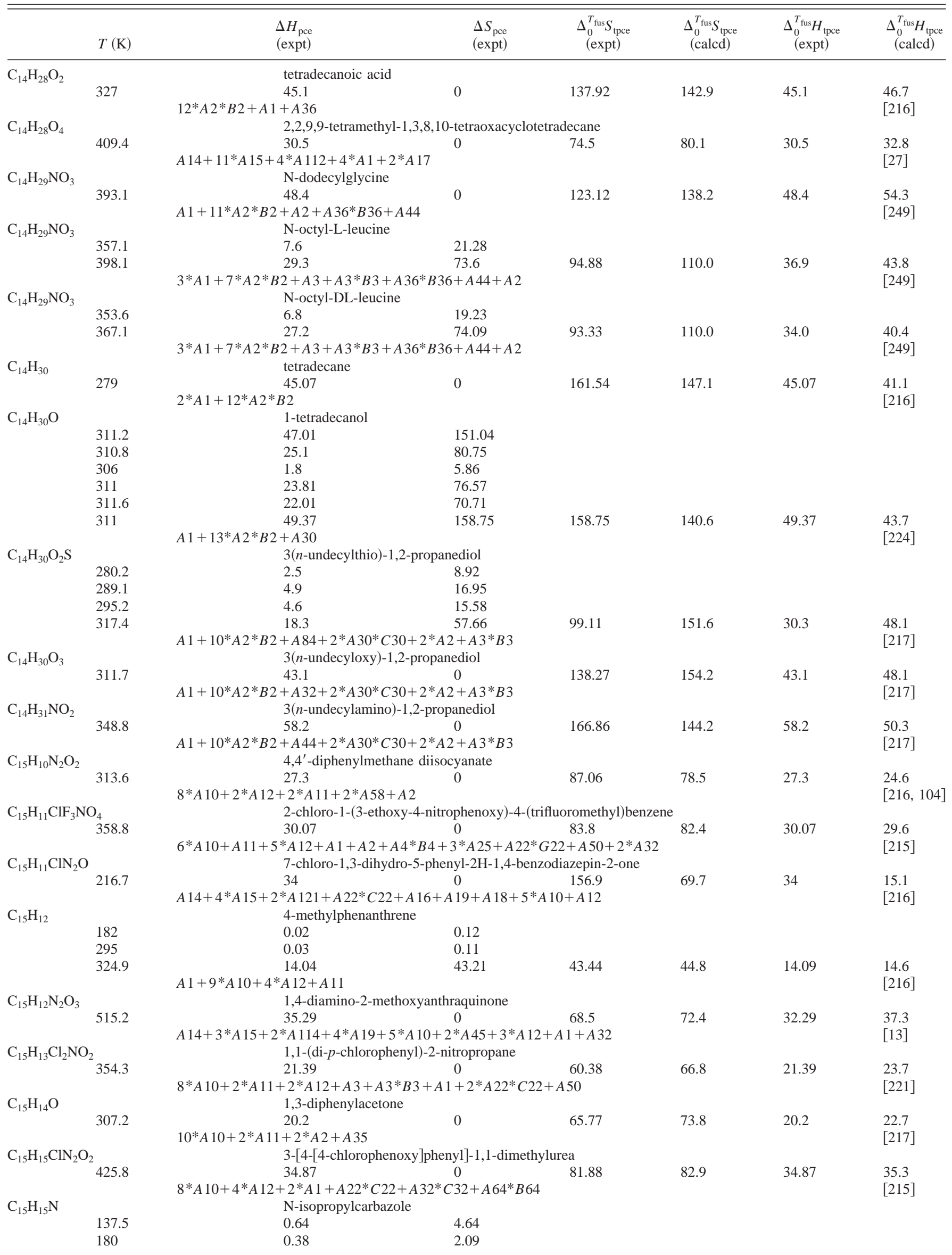


Table 5. Experimental and calculated total phase change enthalpy and entropy of database-Continued

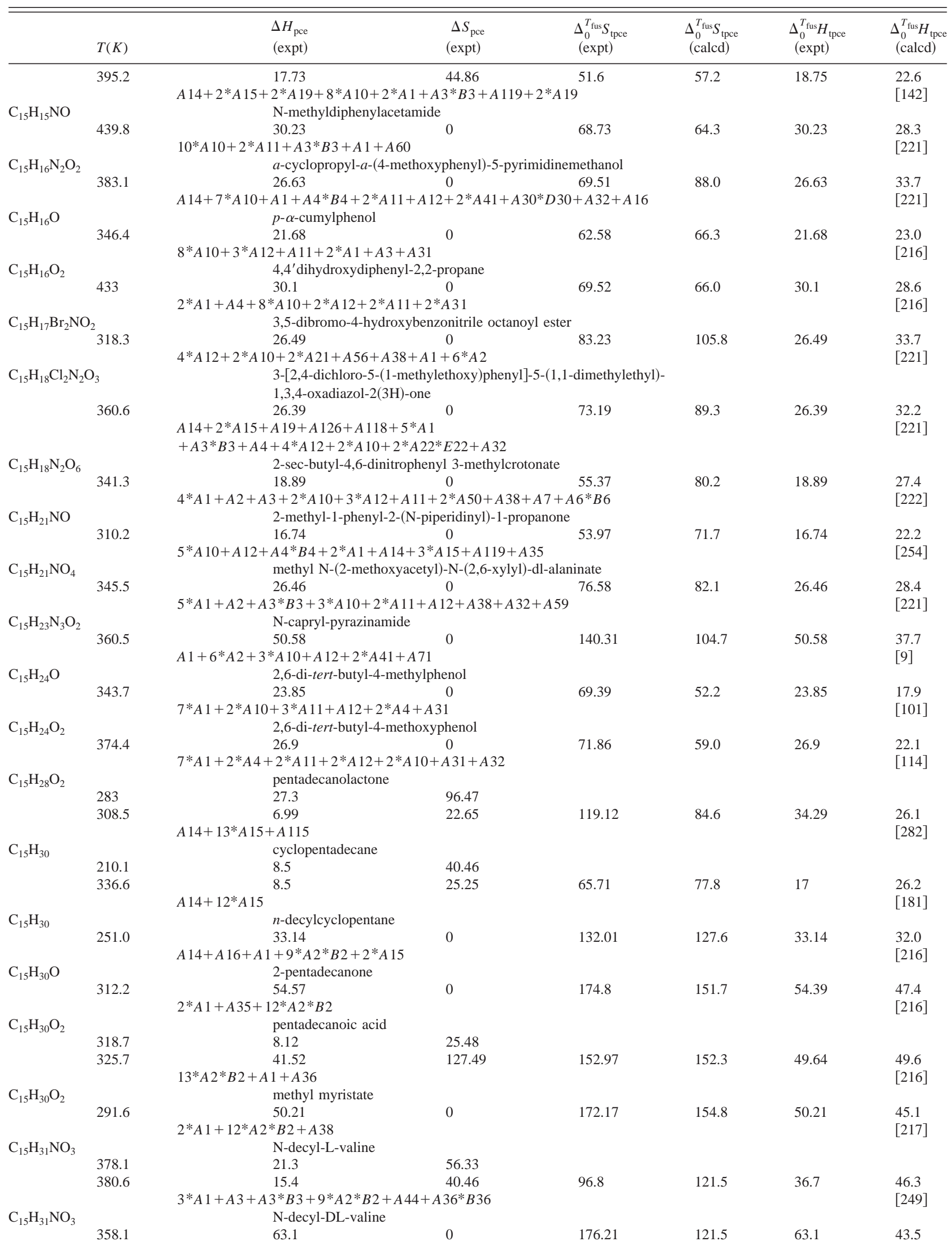


Table 5. Experimental and calculated total phase change enthalpy and entropy of database-Continued

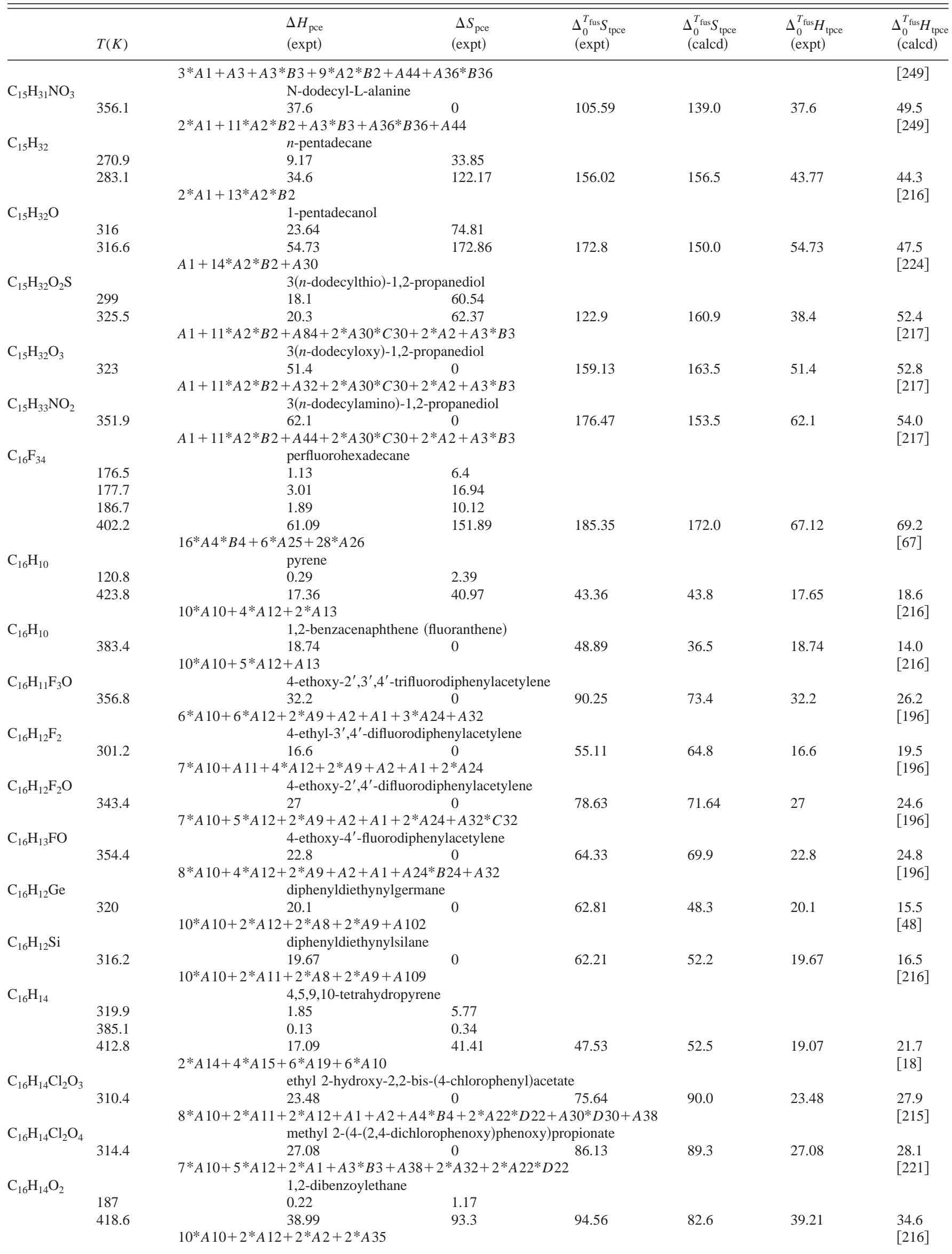


Table 5. Experimental and calculated total phase change enthalpy and entropy of database-Continued

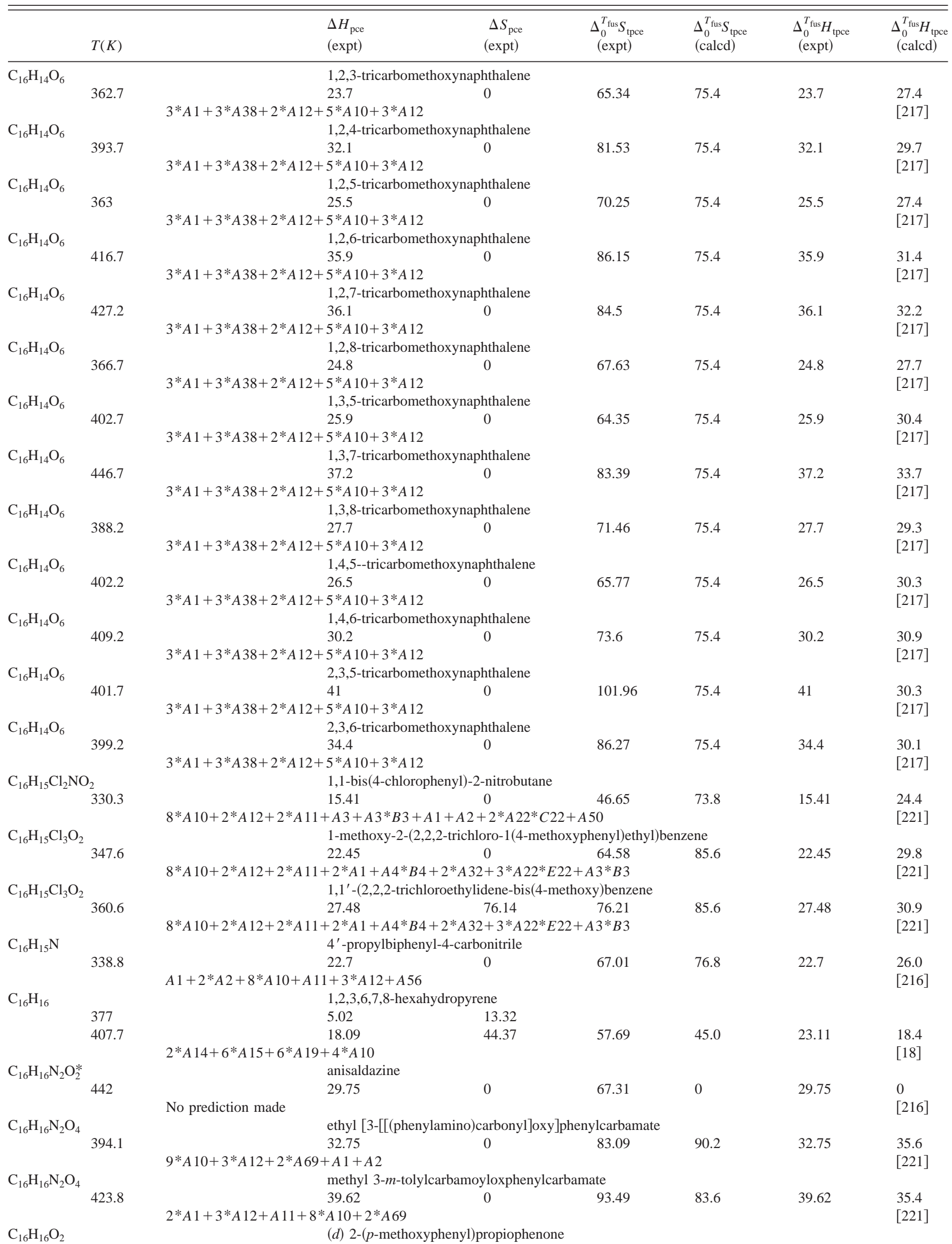


Table 5. Experimental and calculated total phase change enthalpy and entropy of database-Continued

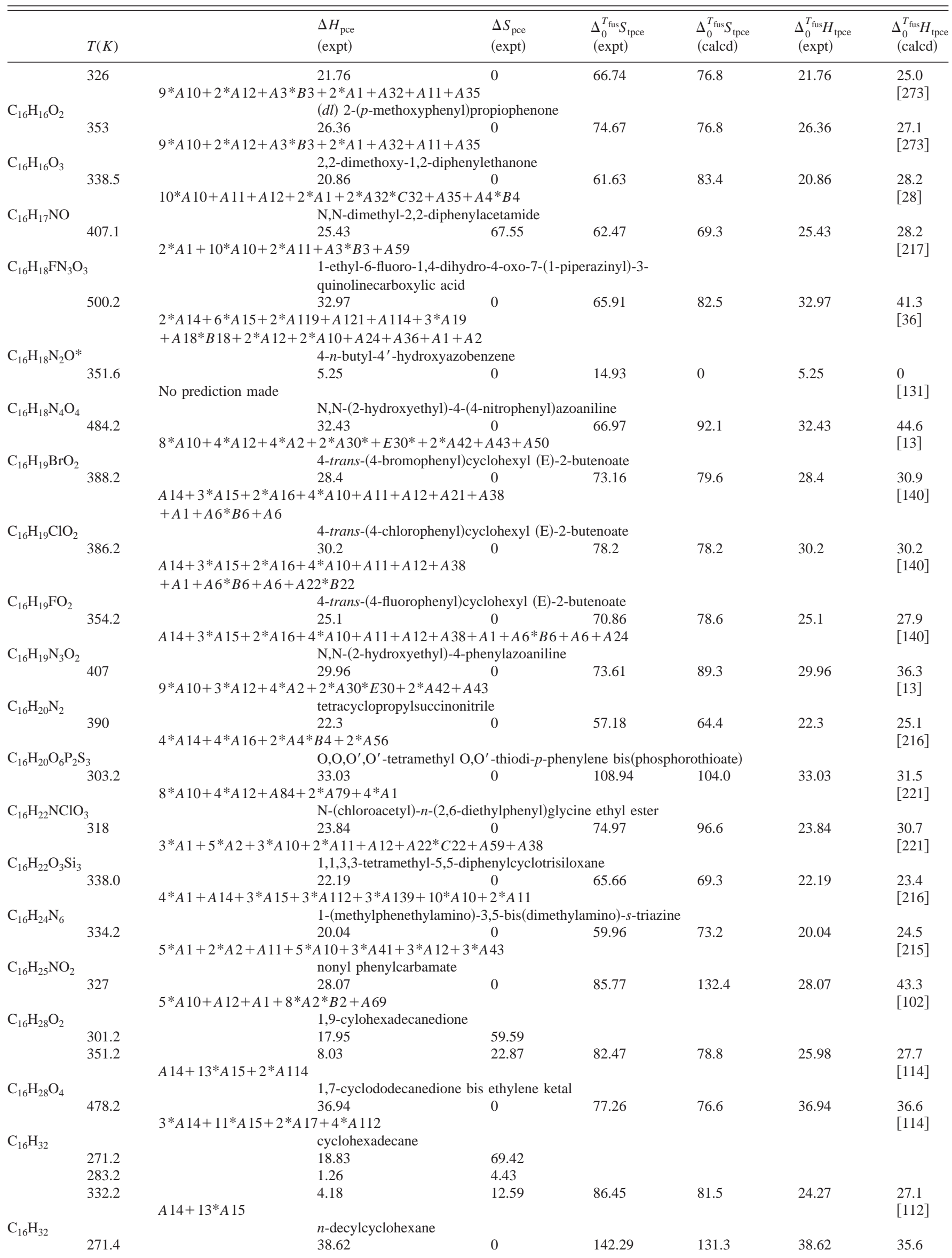


Table 5. Experimental and calculated total phase change enthalpy and entropy of database-Continued

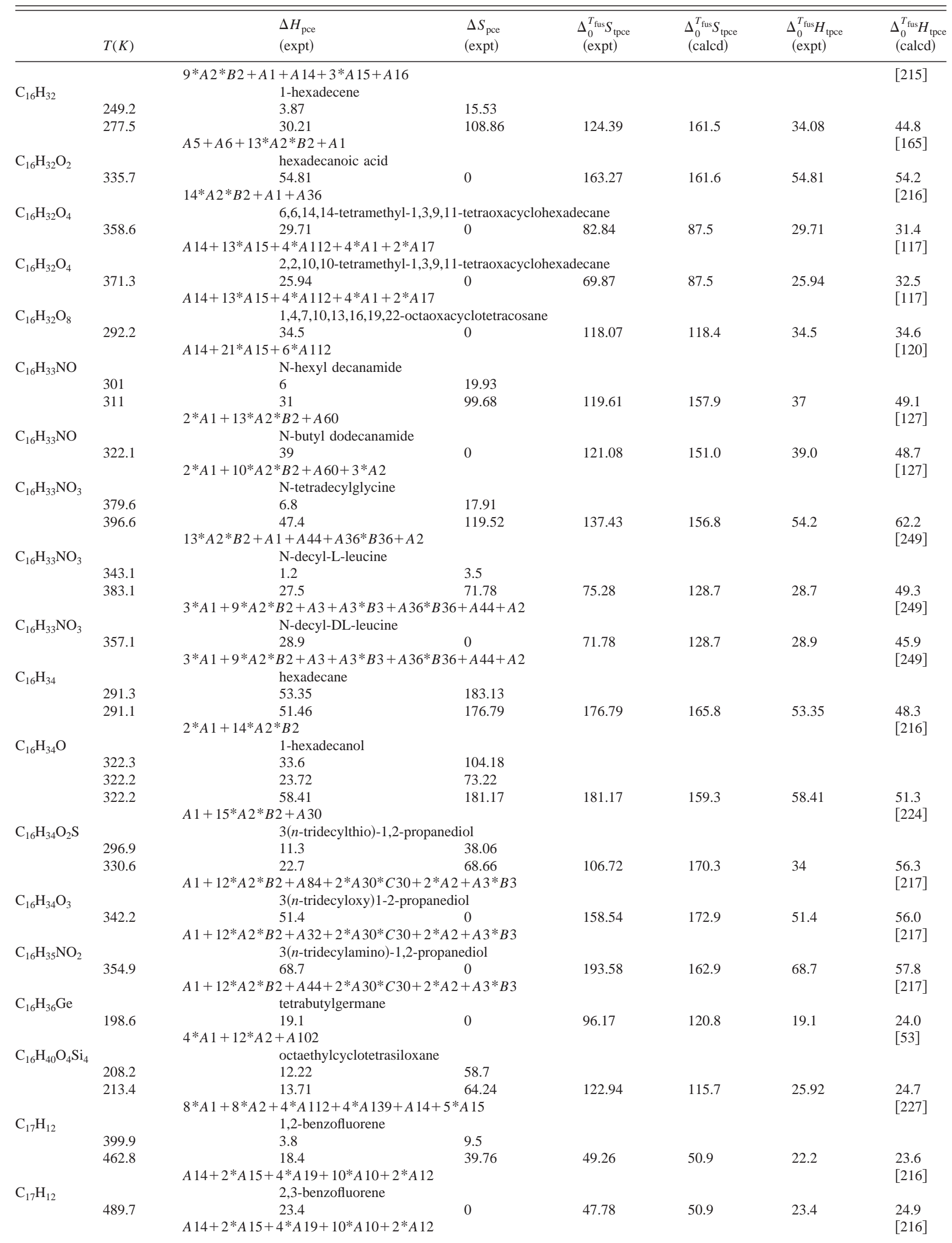


Table 5. Experimental and calculated total phase change enthalpy and entropy of database-Continued

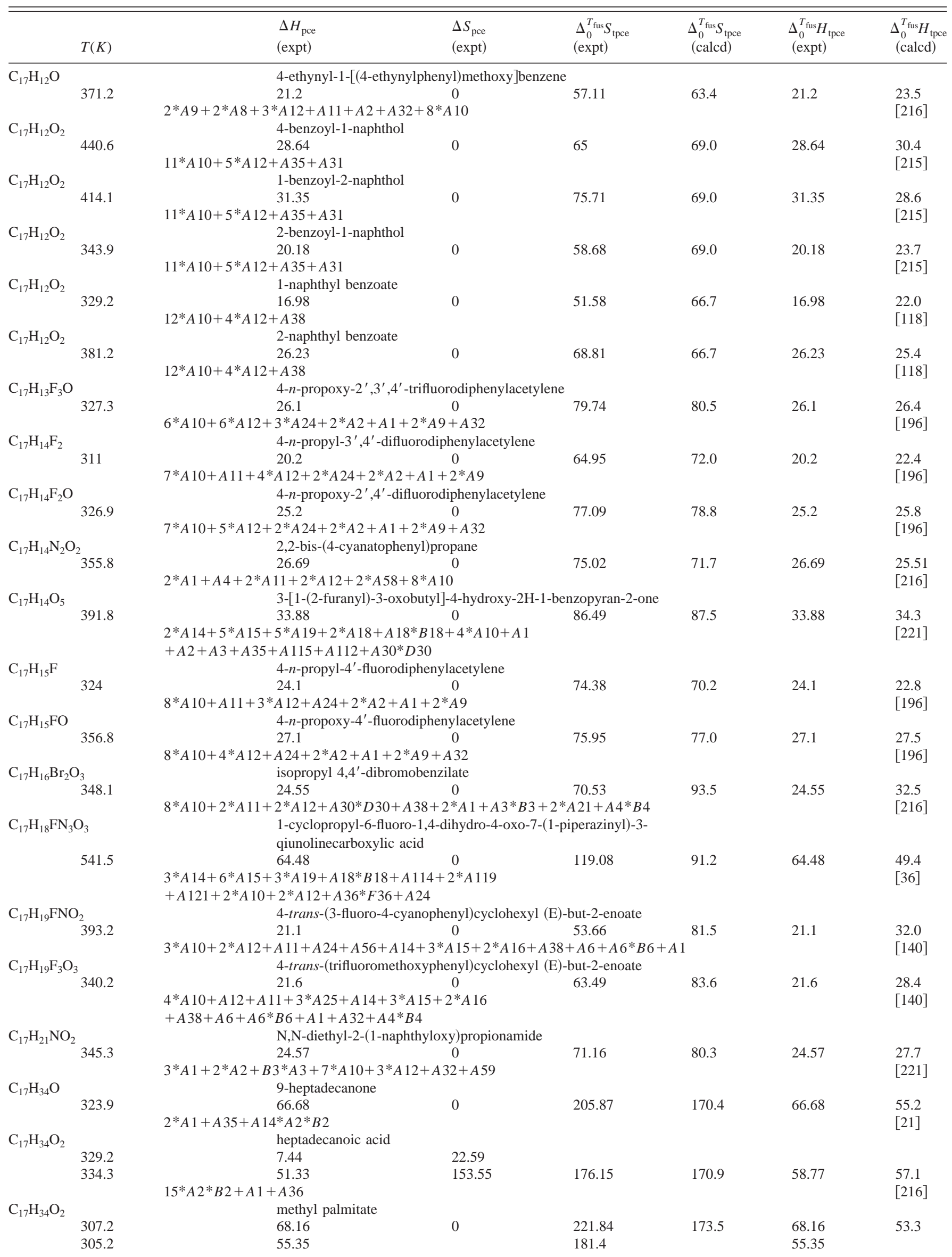


Table 5. Experimental and calculated total phase change enthalpy and entropy of database-Continued

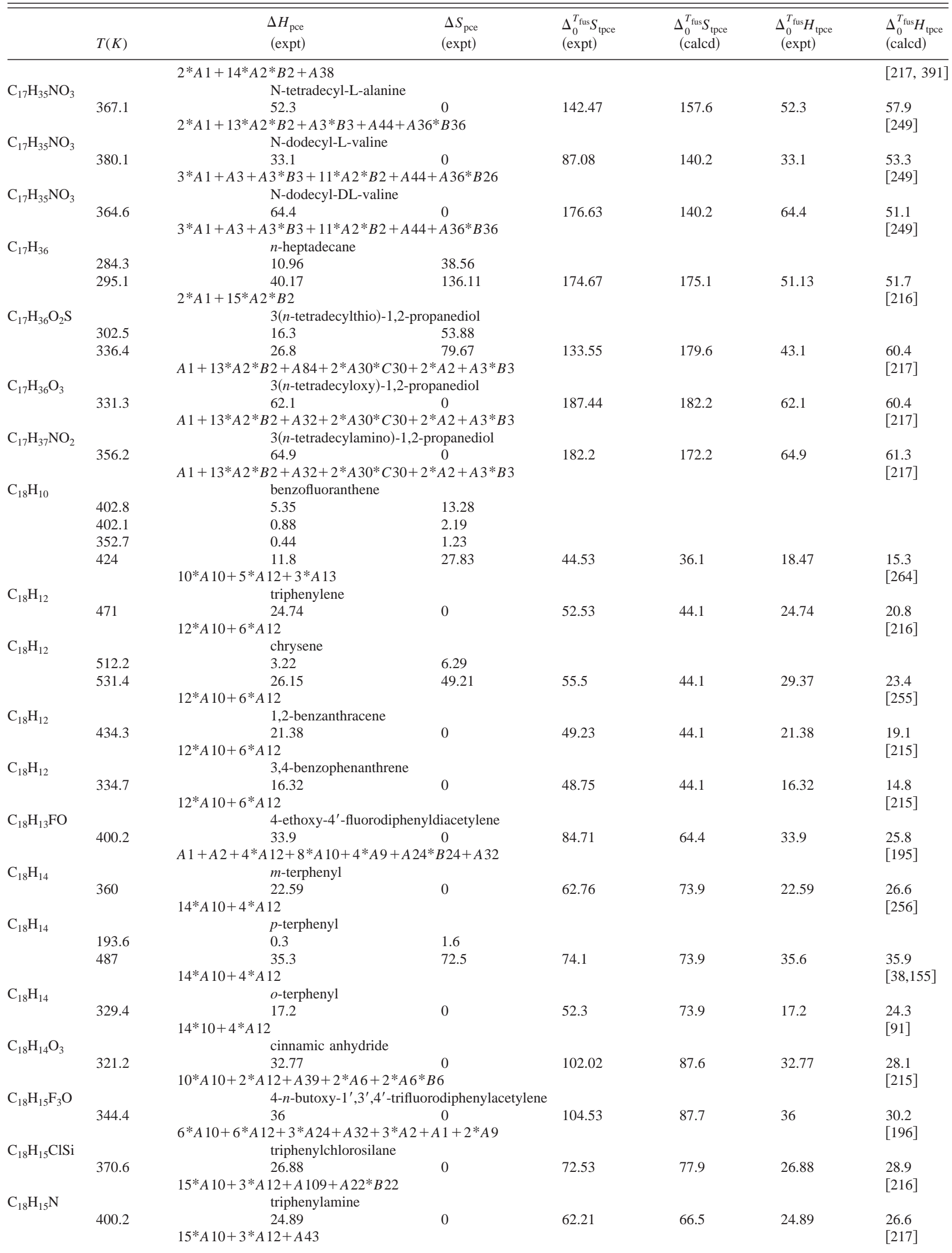


Table 5. Experimental and calculated total phase change enthalpy and entropy of database-Continued

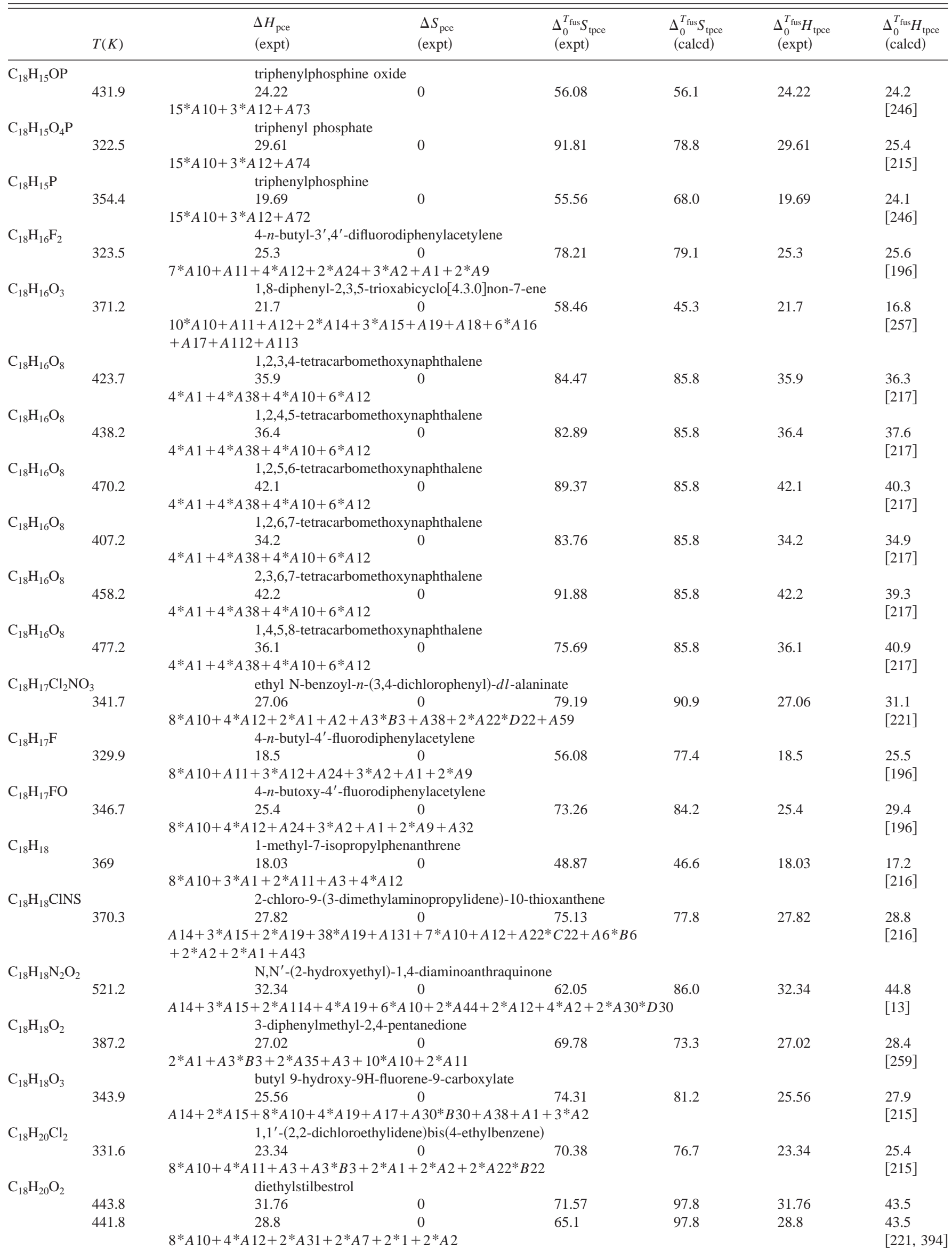


Table 5. Experimental and calculated total phase change enthalpy and entropy of database-Continued

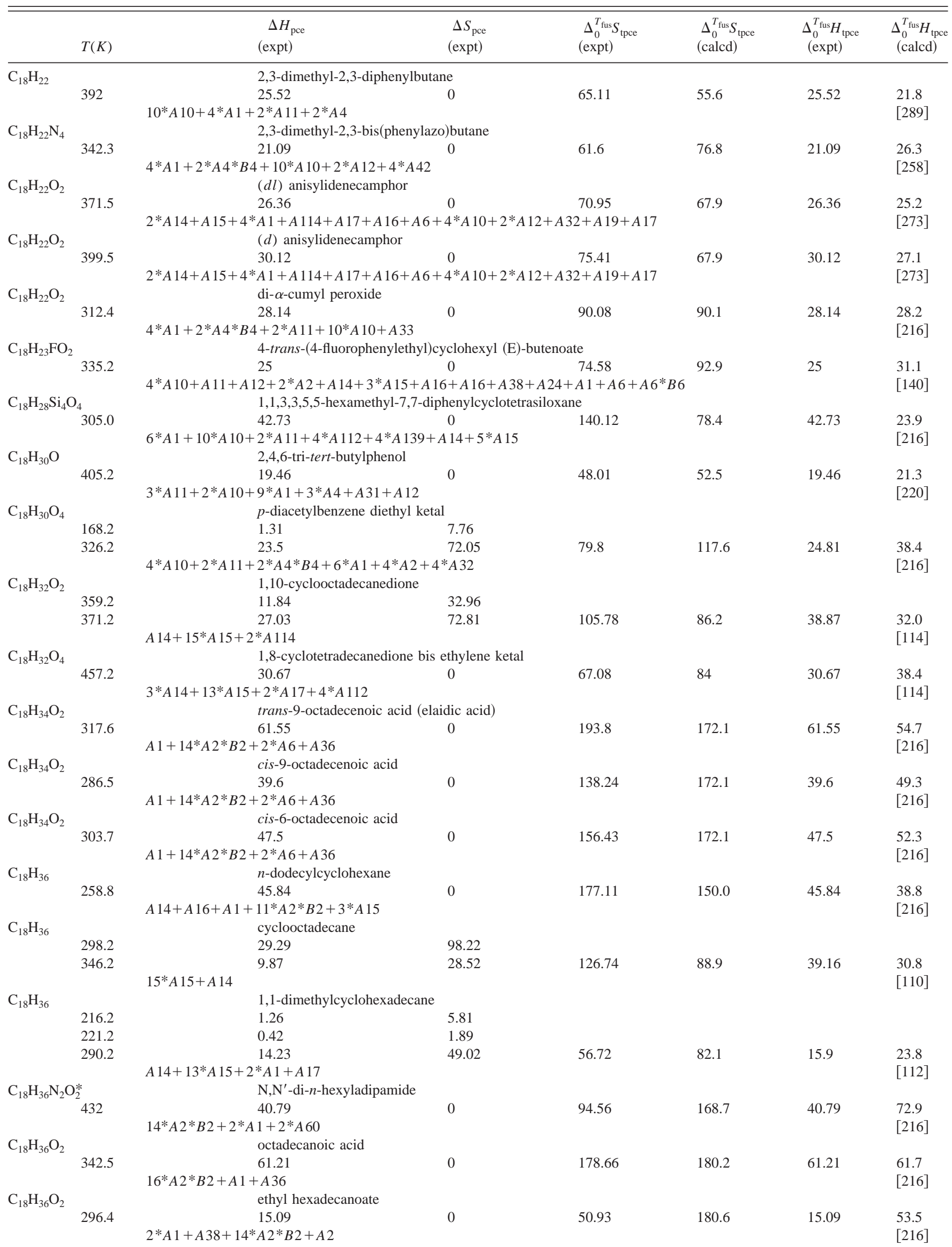


Table 5. Experimental and calculated total phase change enthalpy and entropy of database-Continued

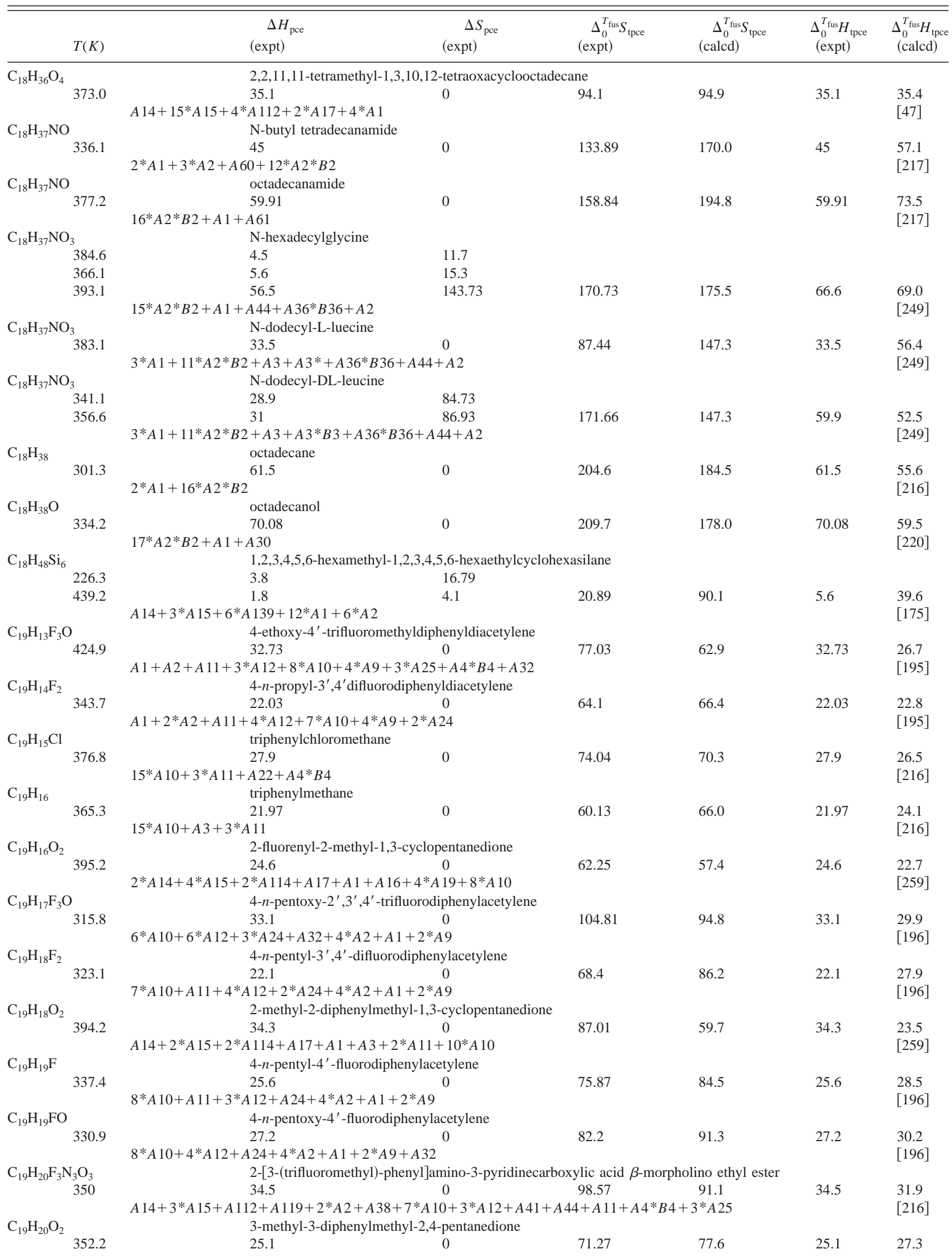


Table 5. Experimental and calculated total phase change enthalpy and entropy of database-Continued

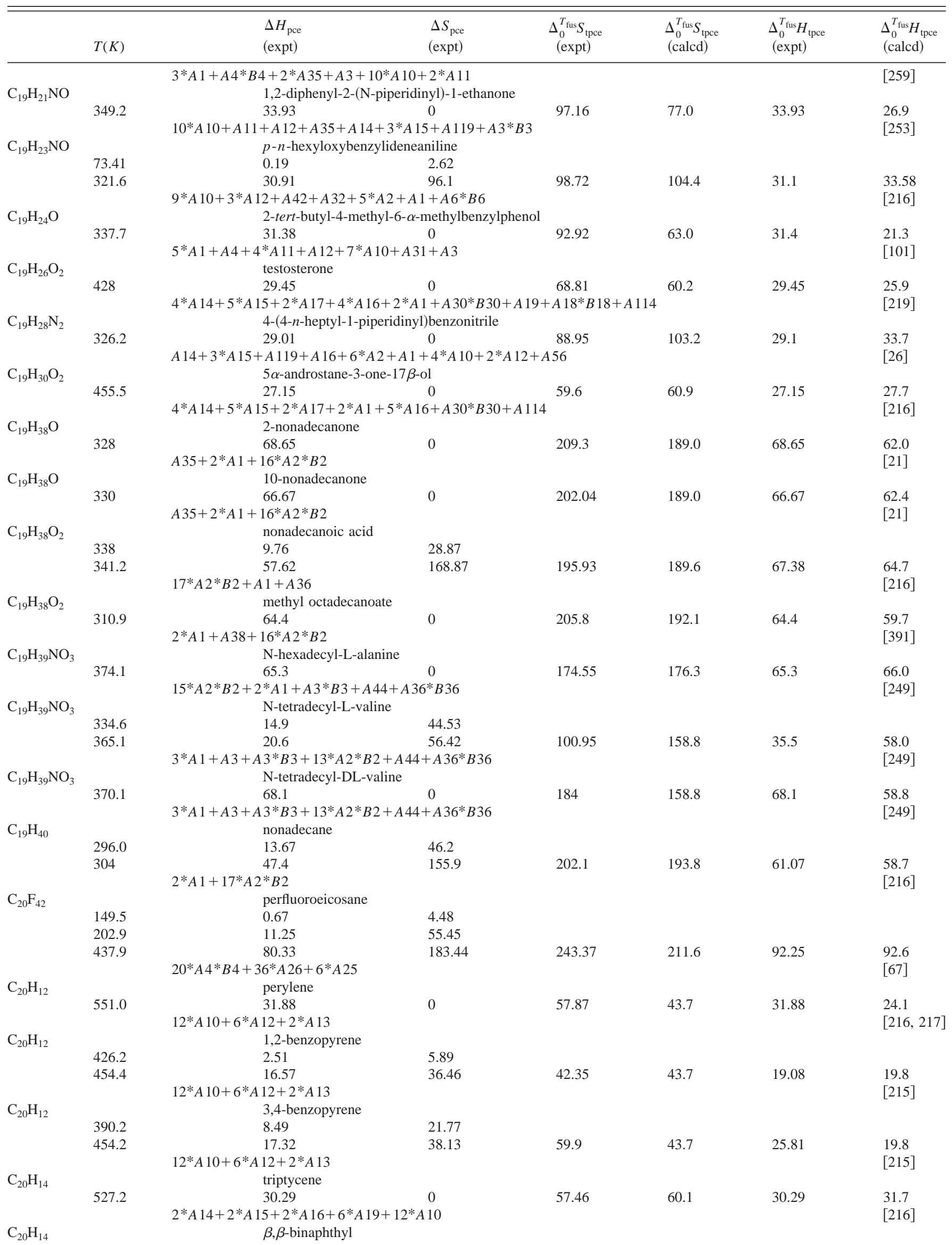


Table 5. Experimental and calculated total phase change enthalpy and entropy of database-Continued

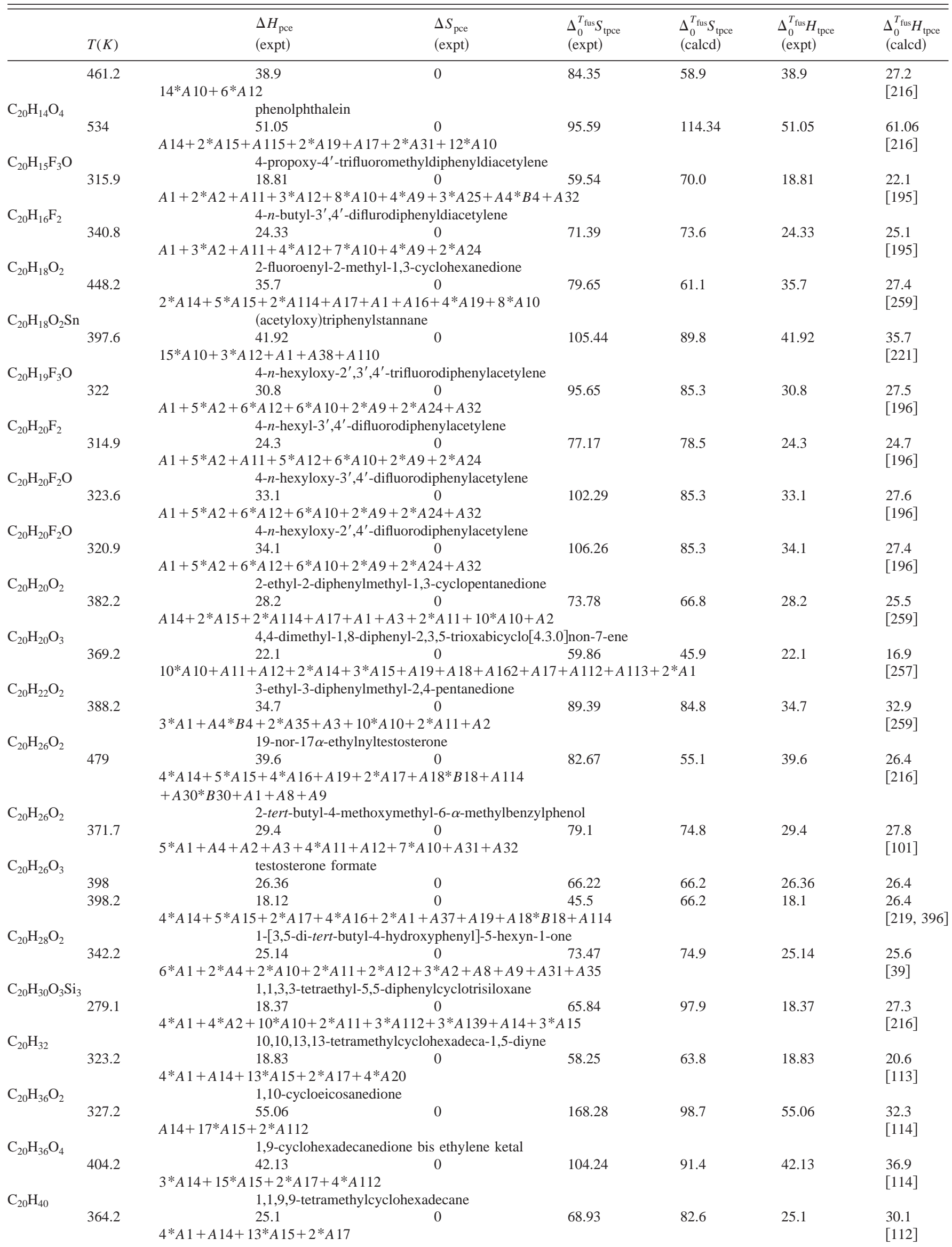


Table 5. Experimental and calculated total phase change enthalpy and entropy of database-Continued

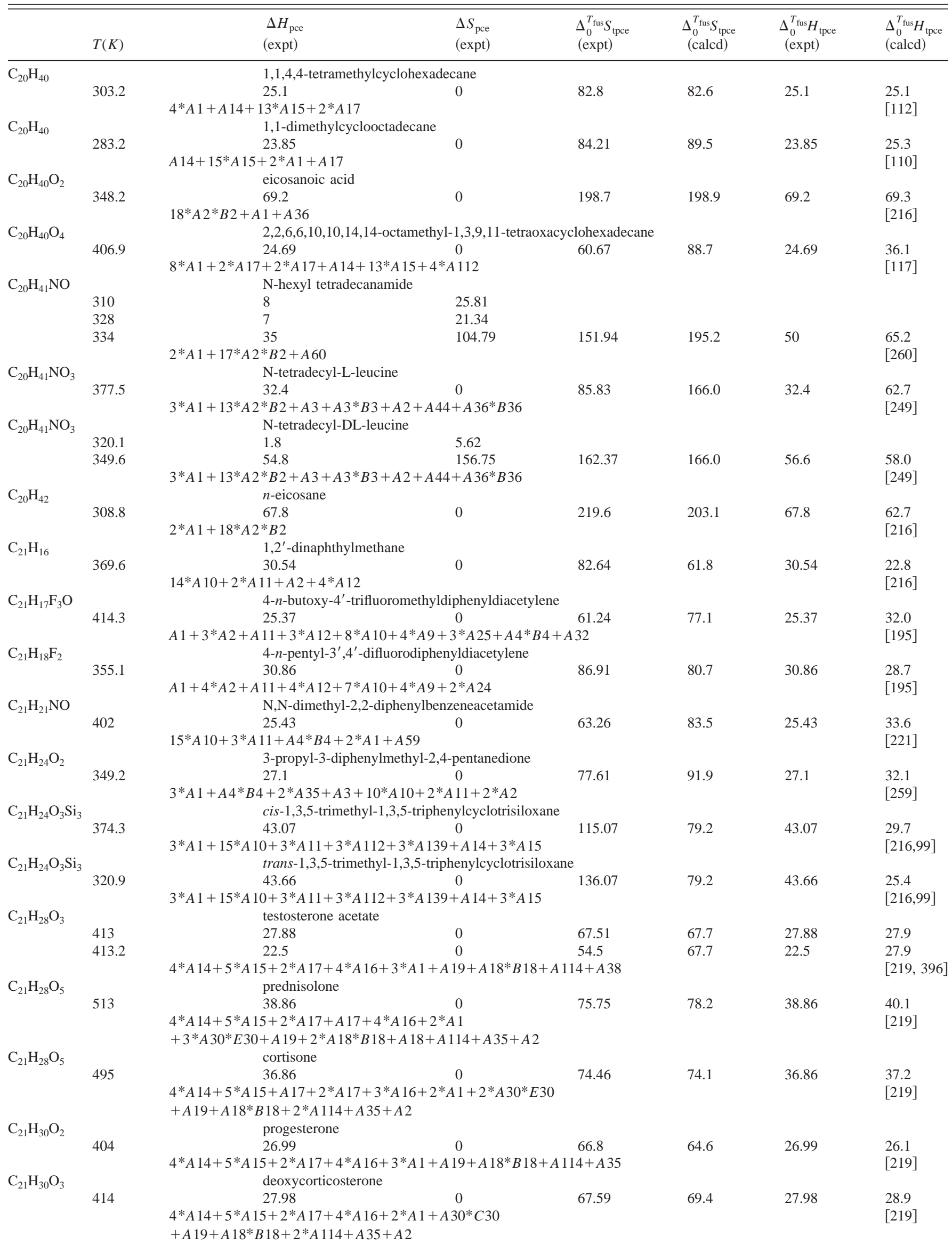


Table 5. Experimental and calculated total phase change enthalpy and entropy of database-Continued

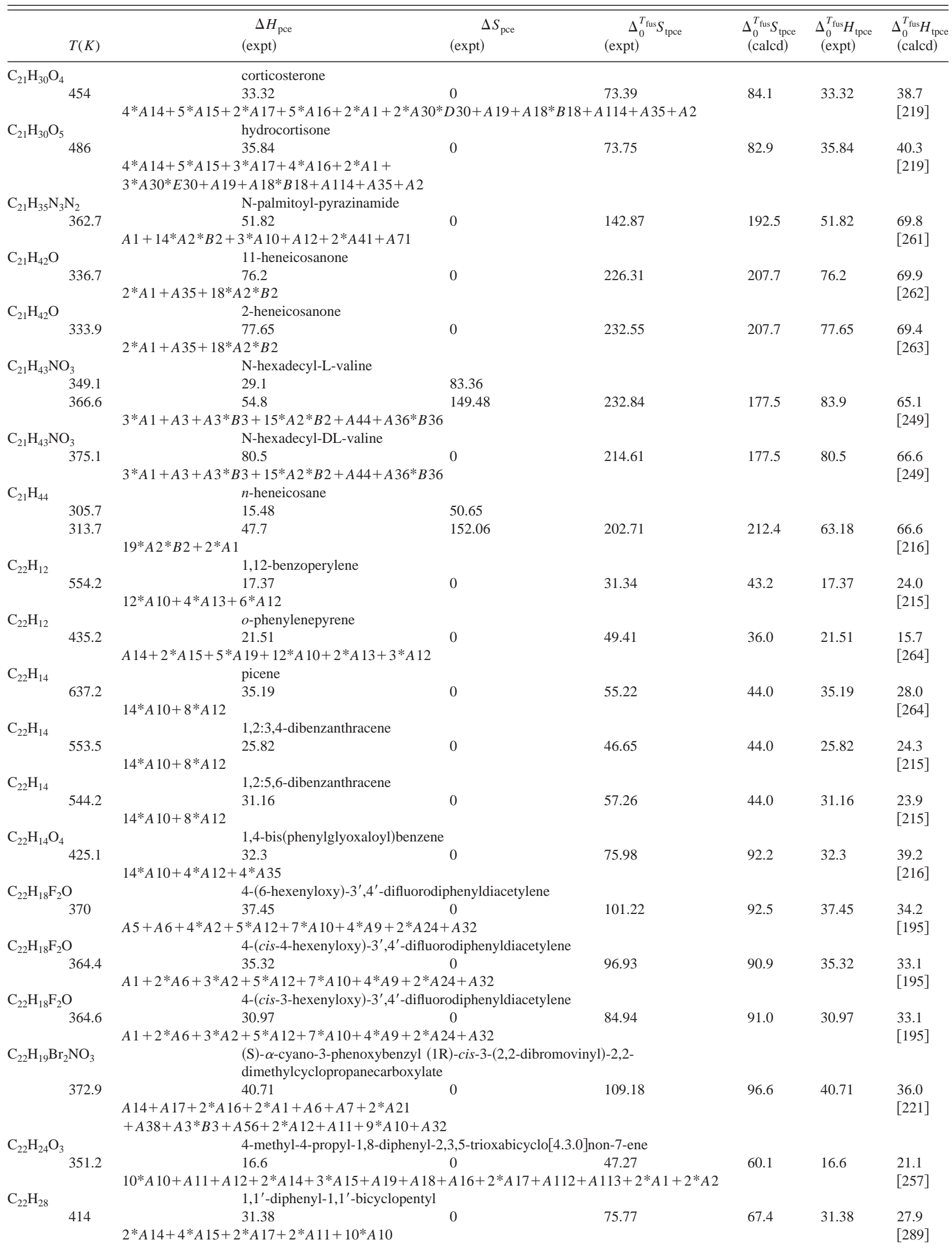


Table 5. Experimental and calculated total phase change enthalpy and entropy of database-Continued

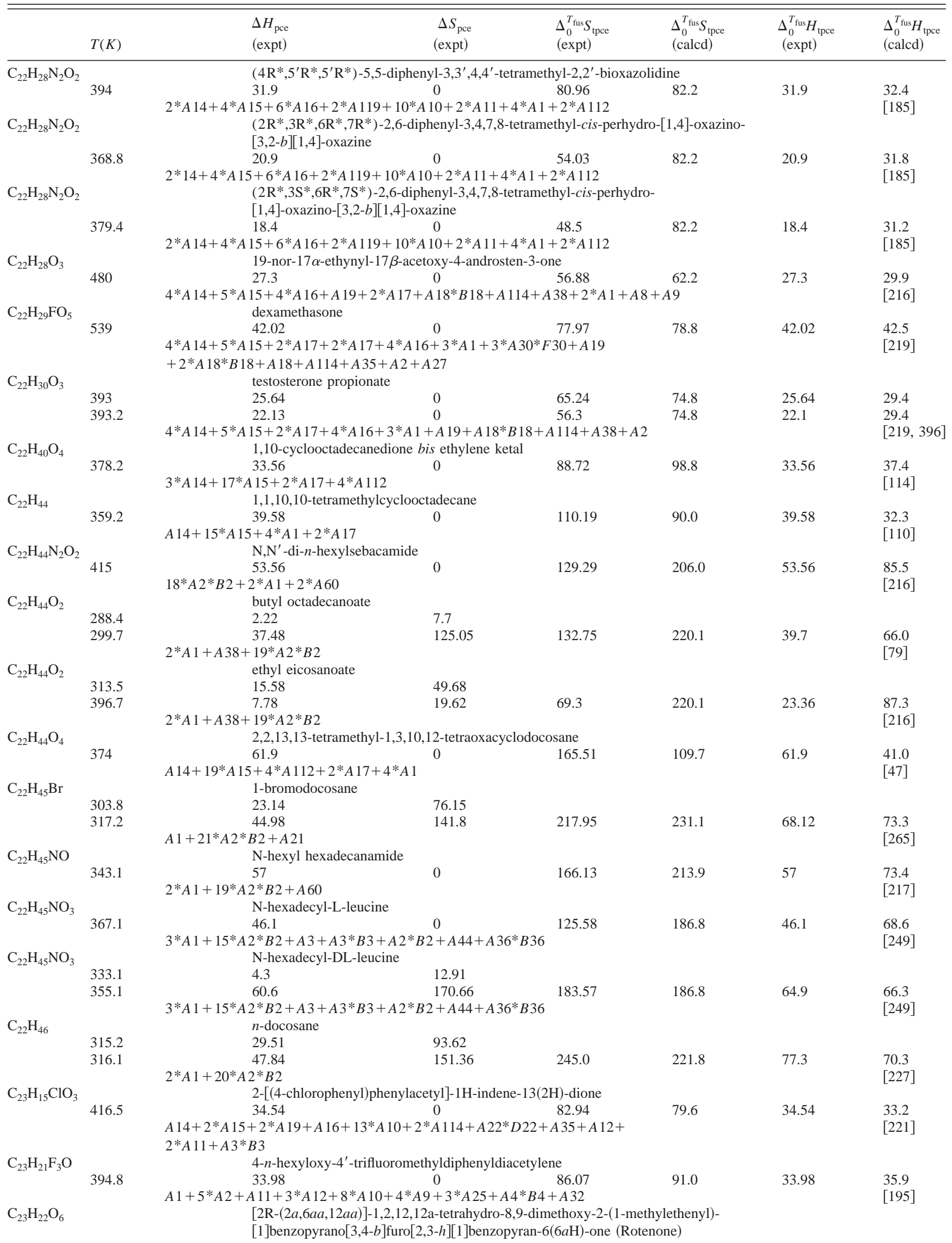


Table 5. Experimental and calculated total phase change enthalpy and entropy of database-Continued

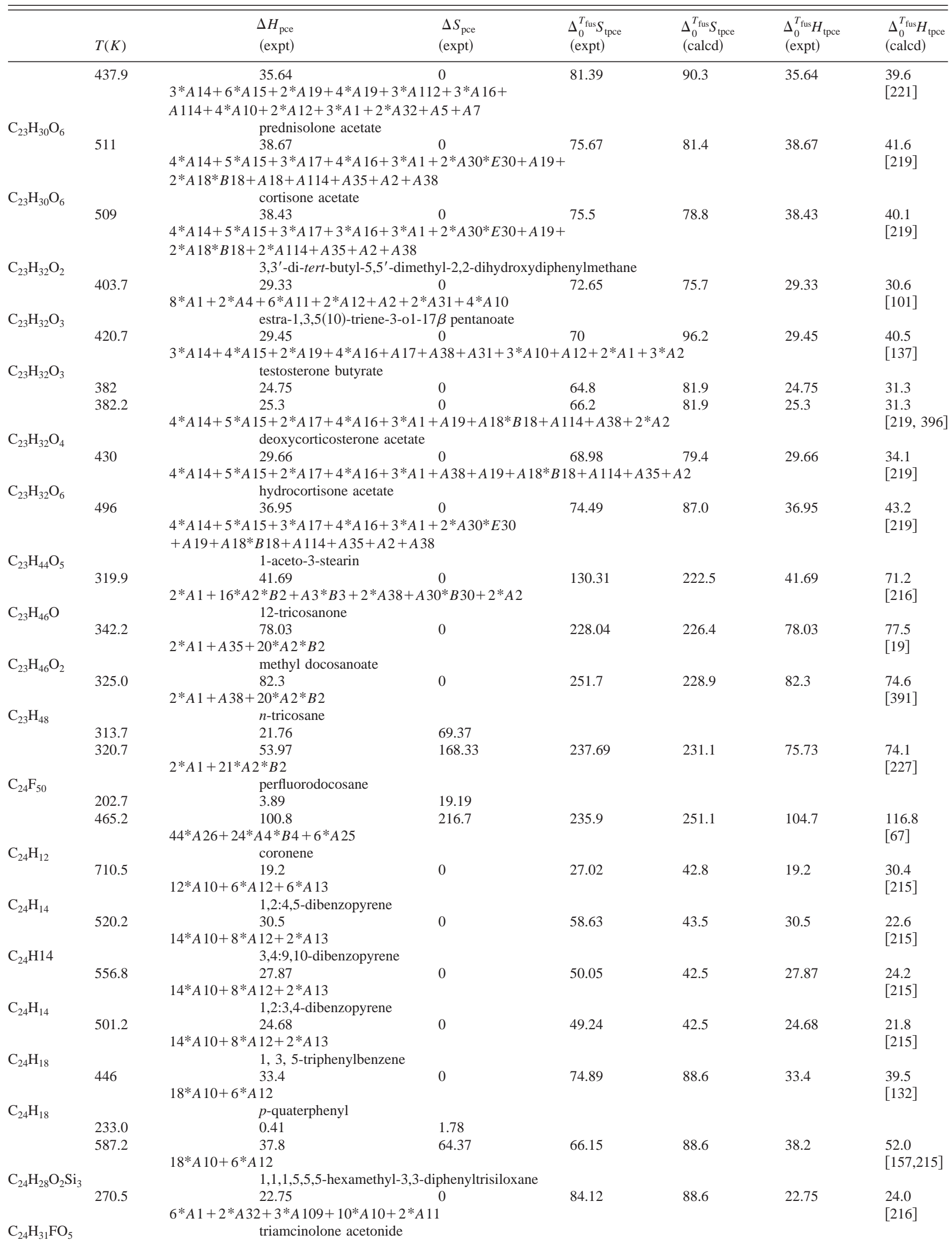


Table 5. Experimental and calculated total phase change enthalpy and entropy of database-Continued

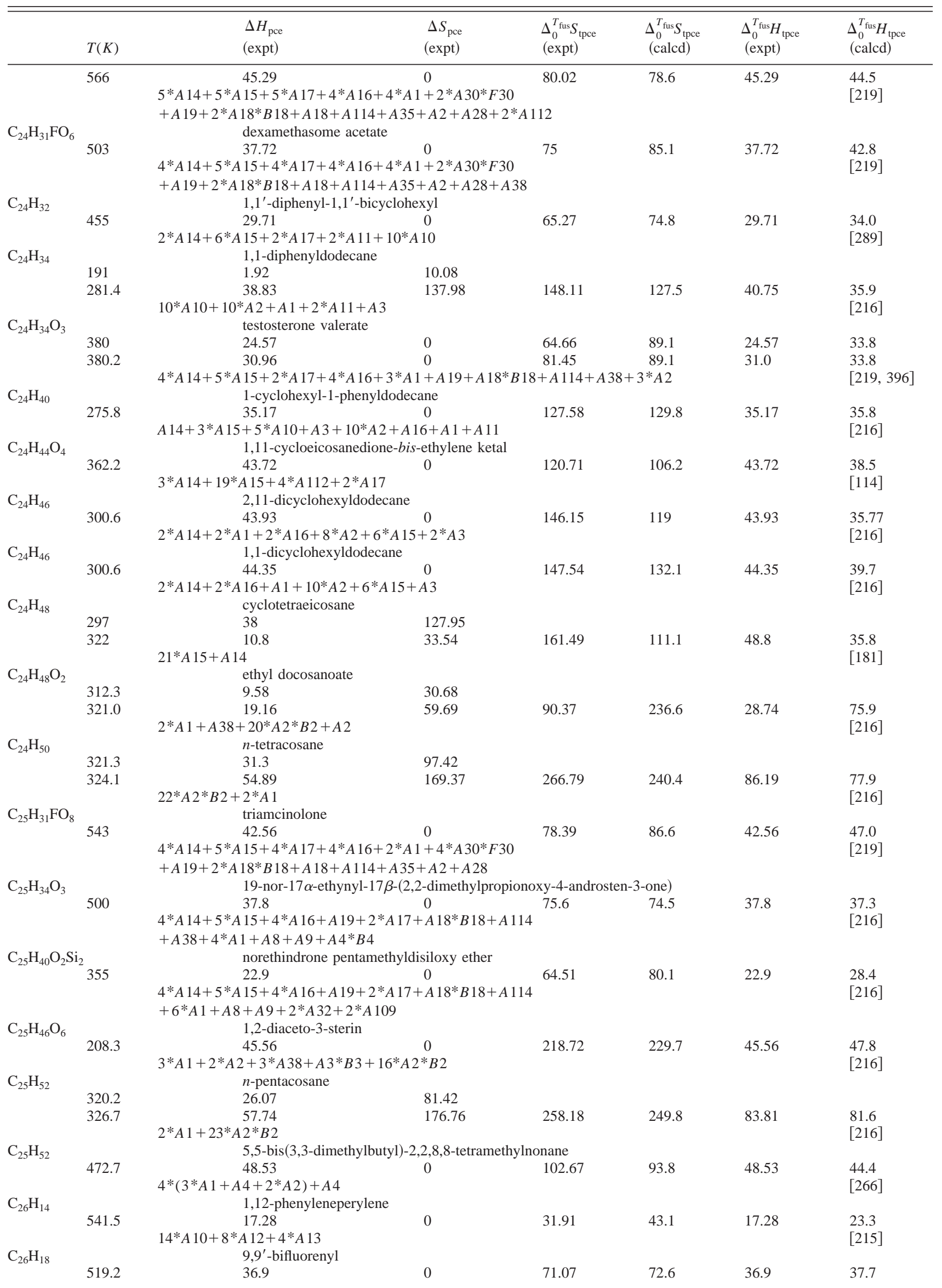


Table 5. Experimental and calculated total phase change enthalpy and entropy of database-Continued

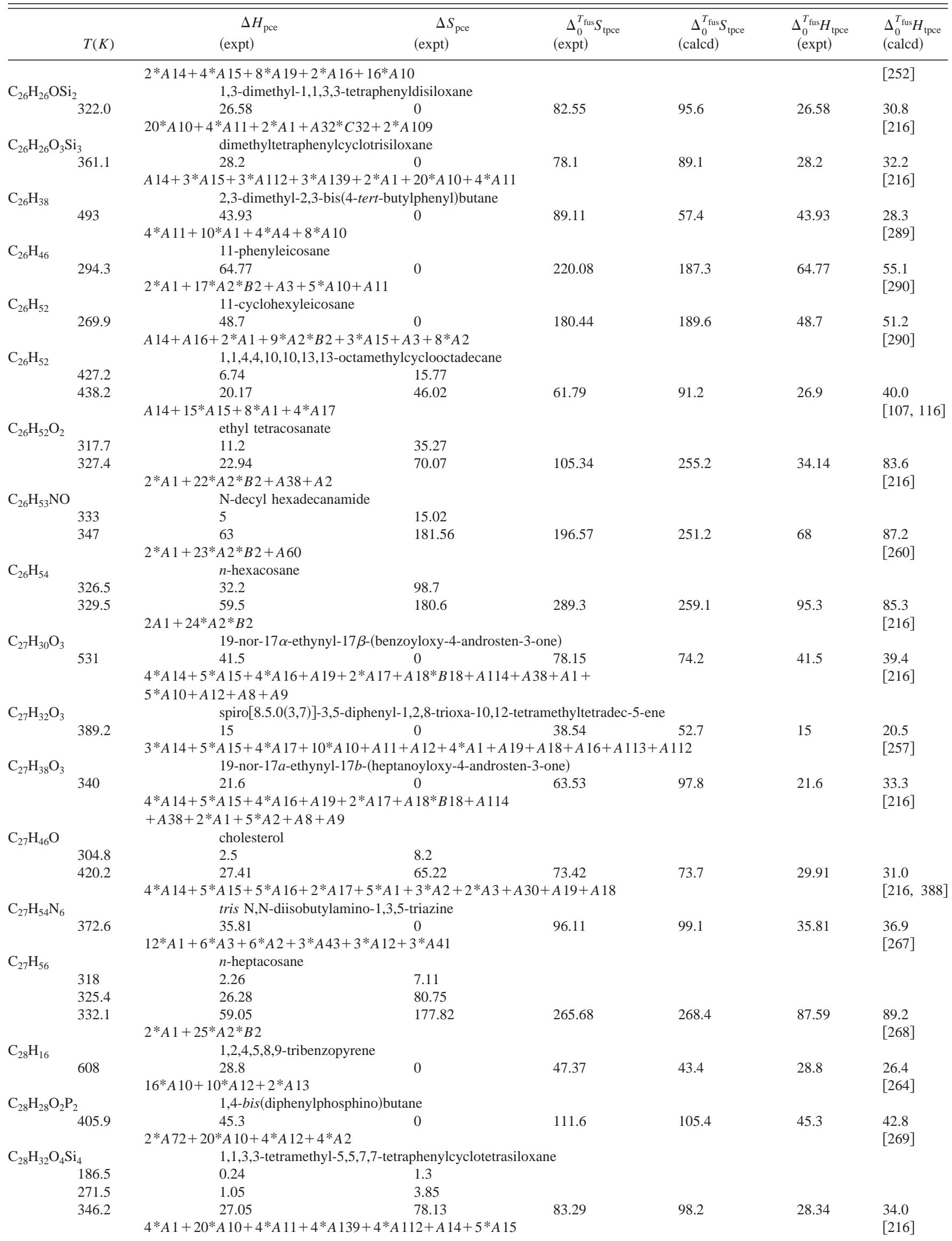


Table 5. Experimental and calculated total phase change enthalpy and entropy of database-Continued

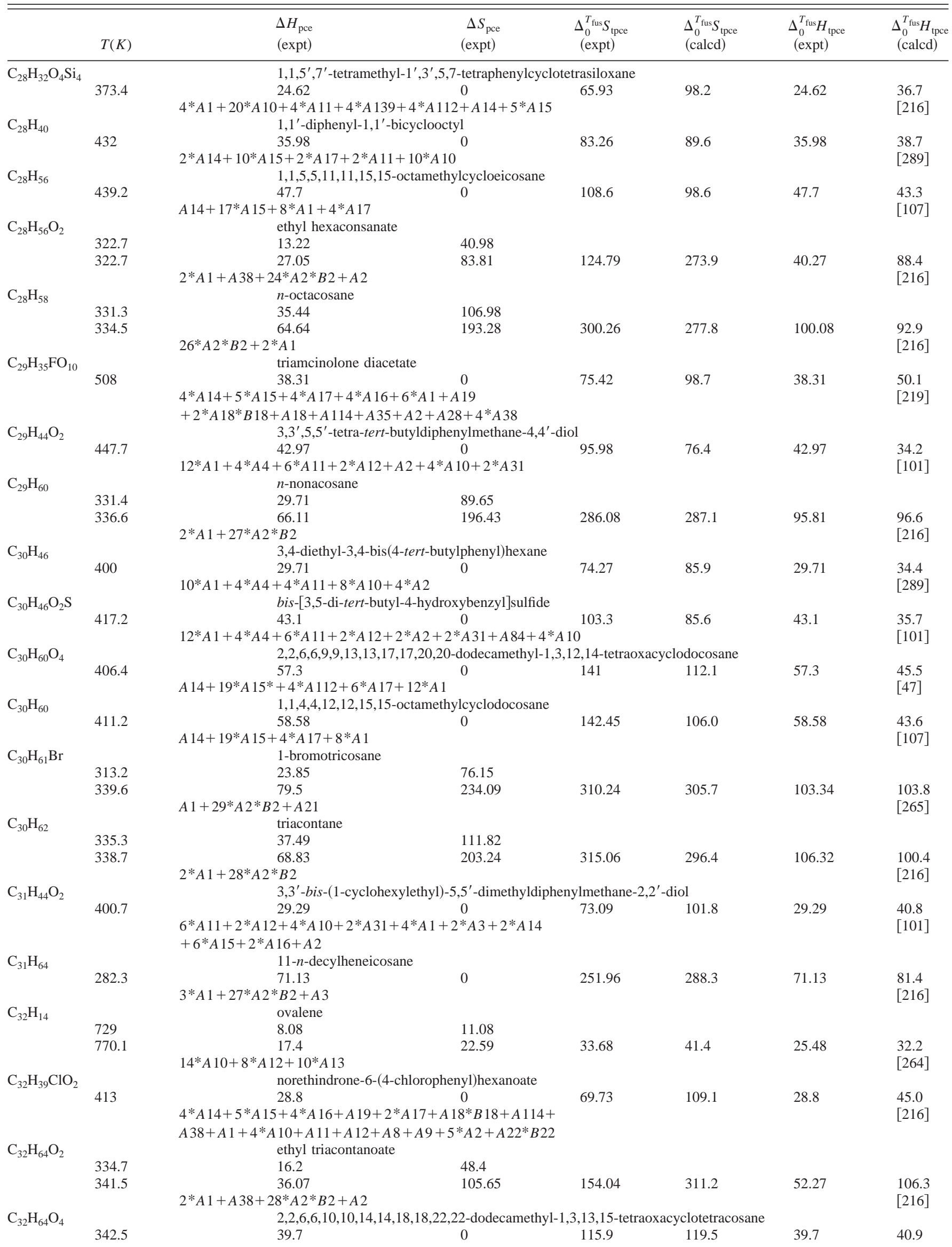


Table 5. Experimental and calculated total phase change enthalpy and entropy of database-Continued

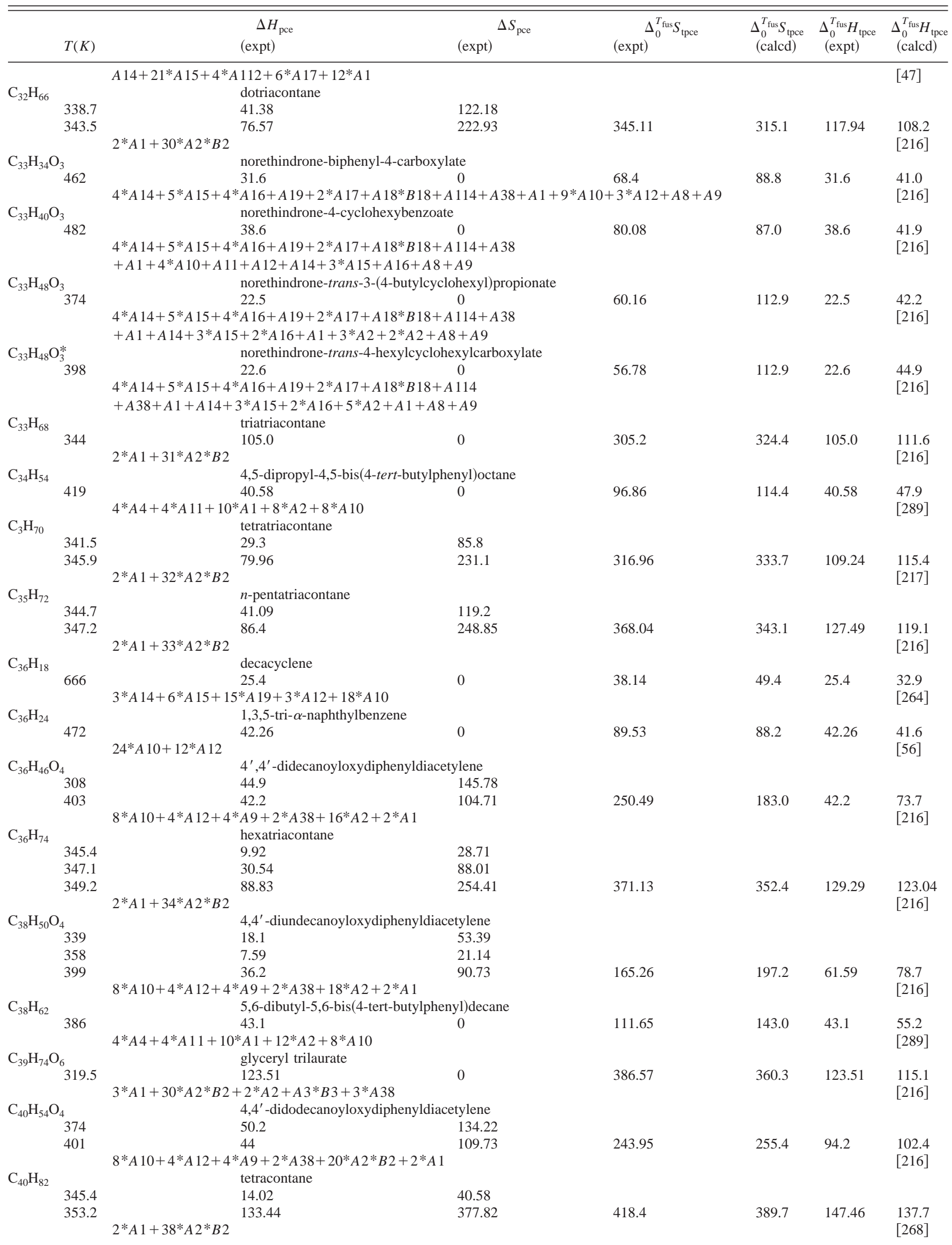


Table 5. Experimental and calculated total phase change enthalpy and entropy of database-Continued

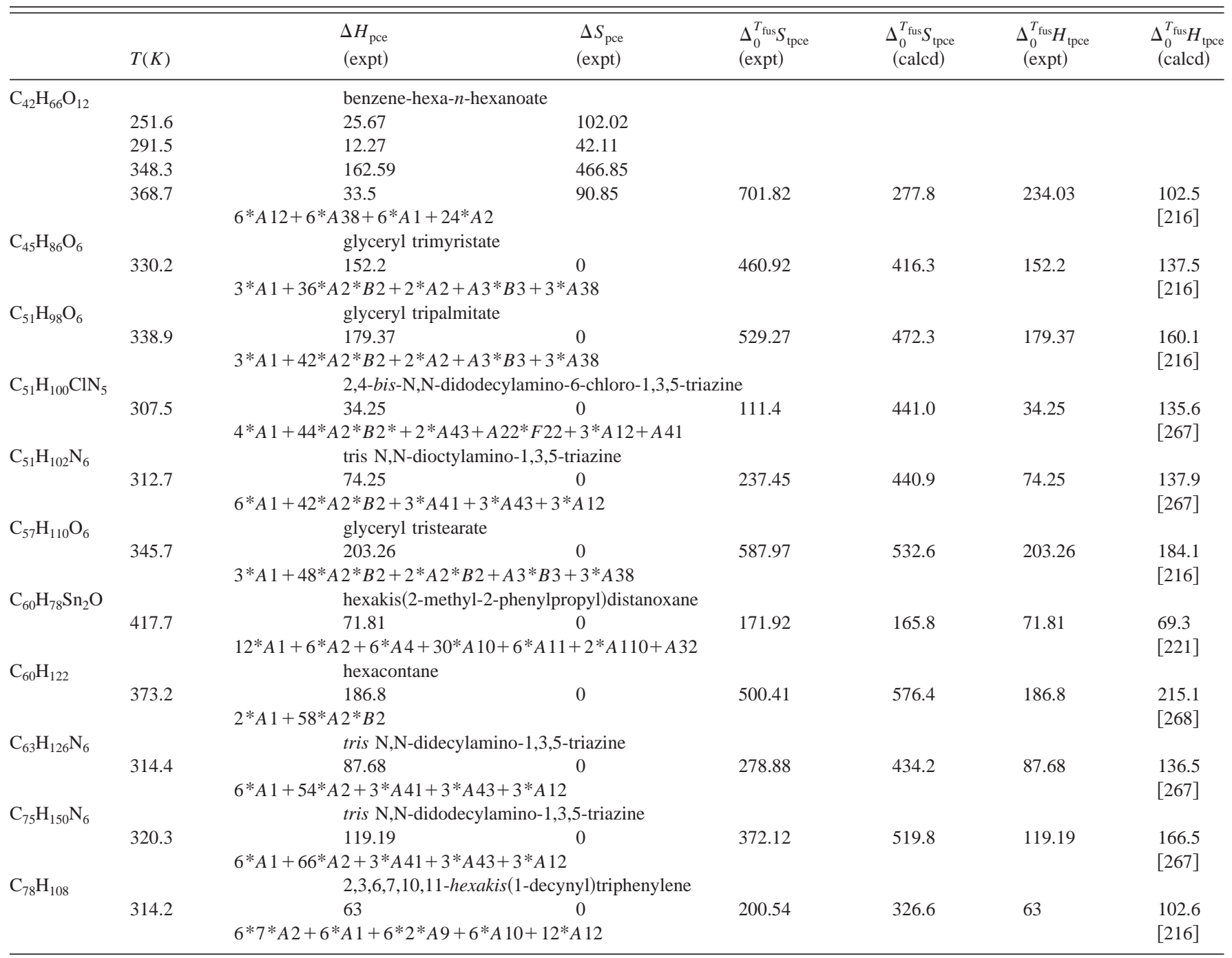

${ }^{a}$ Units for $\Delta_{0}^{T_{\text {fus }}} S_{\text {tpce }}$ and $\Delta_{0}^{T_{\text {fus }}} H_{\text {tpce }}$ are $\mathrm{J} \cdot \mathrm{mol}^{-1} \cdot \mathrm{K}^{-1}$ and $\mathrm{kJ} \cdot \mathrm{mol}^{-1}$, respectively; compounds with molecular formulas characterized with an asterisk $(*)$ were not included in generating the statistics. As noted in the text, some of these compounds exhibit liquid crystal behavior, others display amphiphillic behavior, group values for some are not currently available or the error between experimental and calculated total phase change entropy exceeded 3 standard deviations. 
TABLE 6. Experimental and calculated total phase change enthalpies and entropies of fusion of polymers ${ }^{\mathrm{a}}$

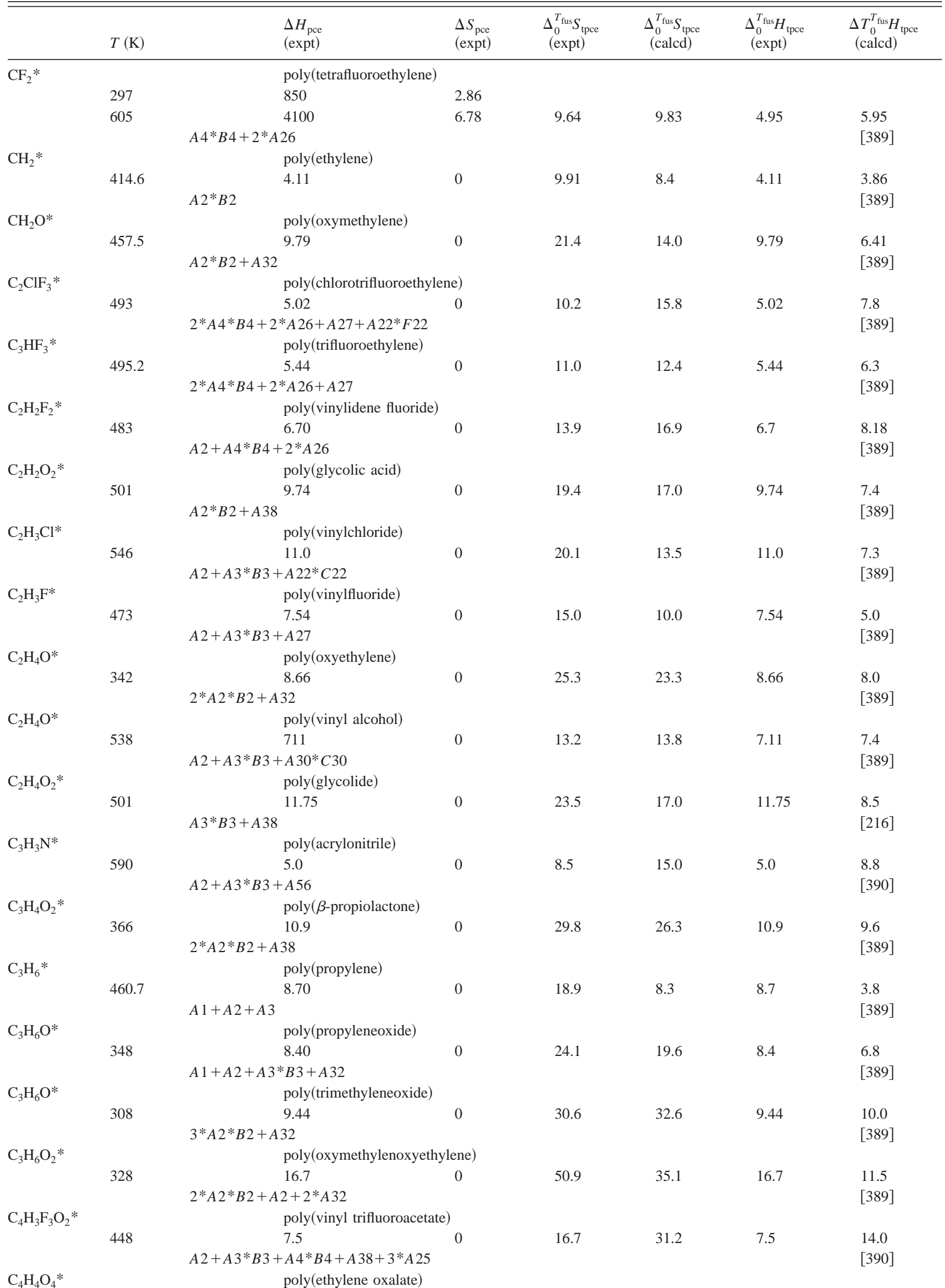


TABLE 6. Experimental and calculated total phase change enthalpies and entropies of fusion of polymers-Continued

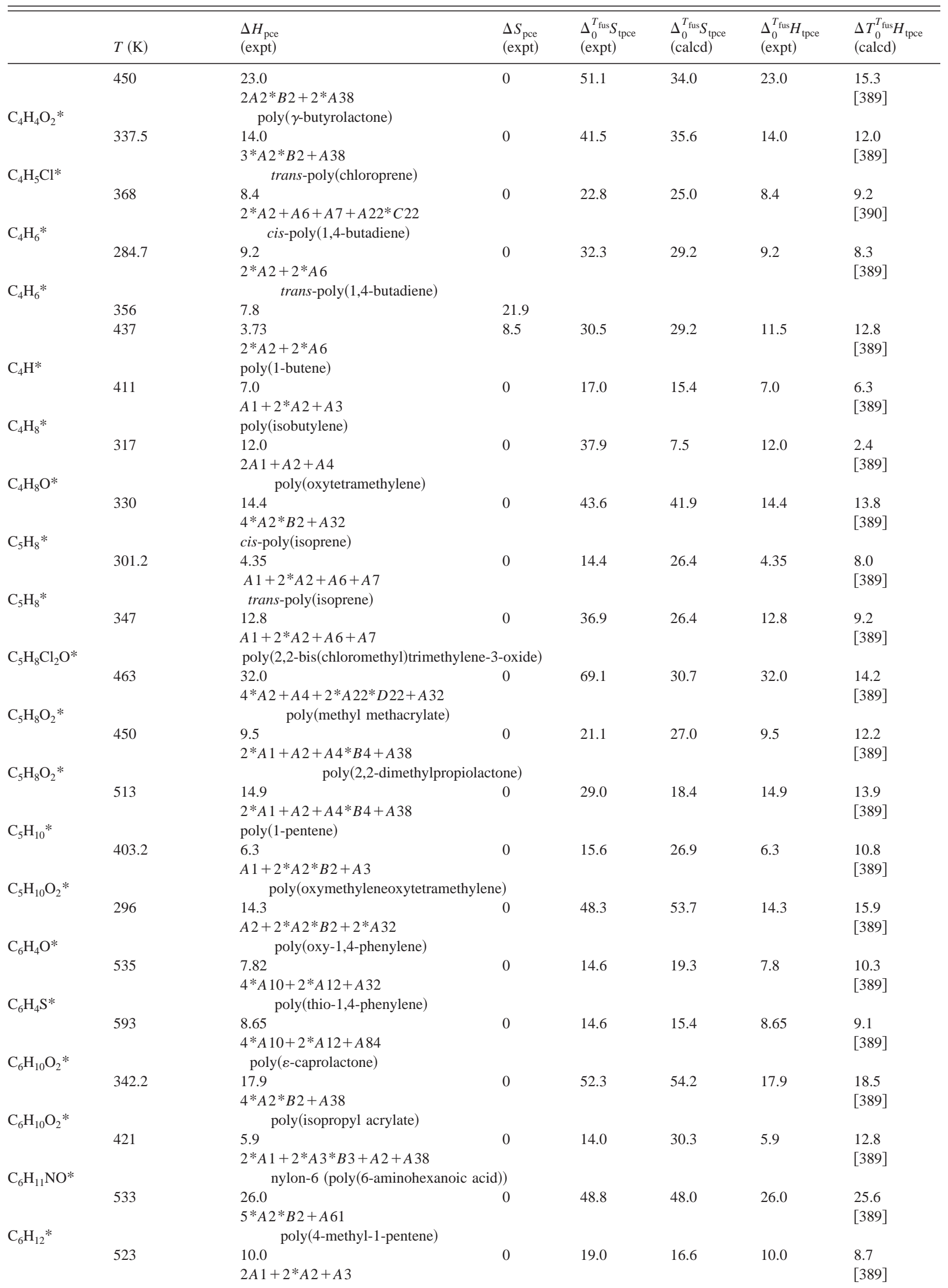


TABLE 6. Experimental and calculated total phase change enthalpies and entropies of fusion of polymers-Continued

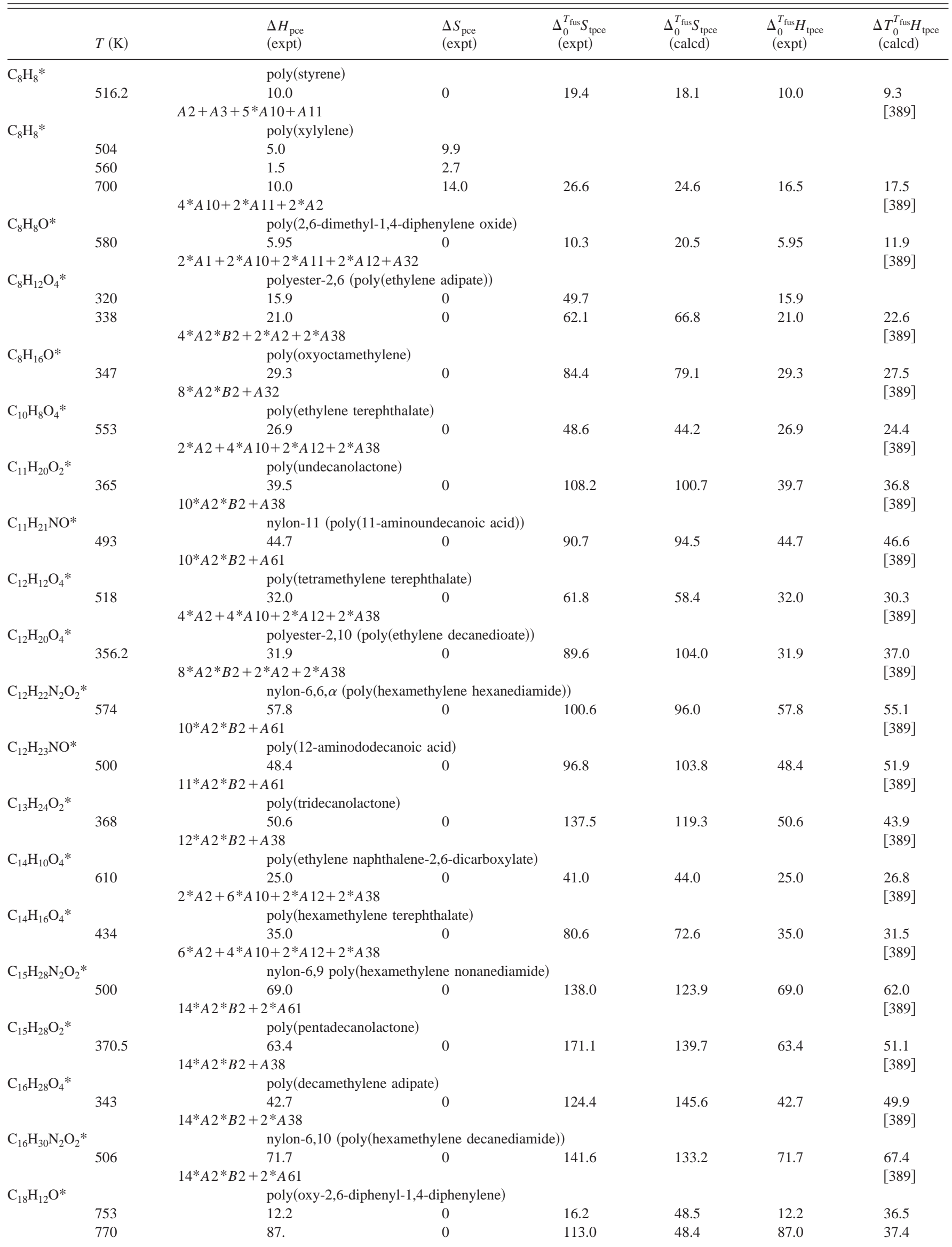


TABLE 6. Experimental and calculated total phase change enthalpies and entropies of fusion of polymers-Continued

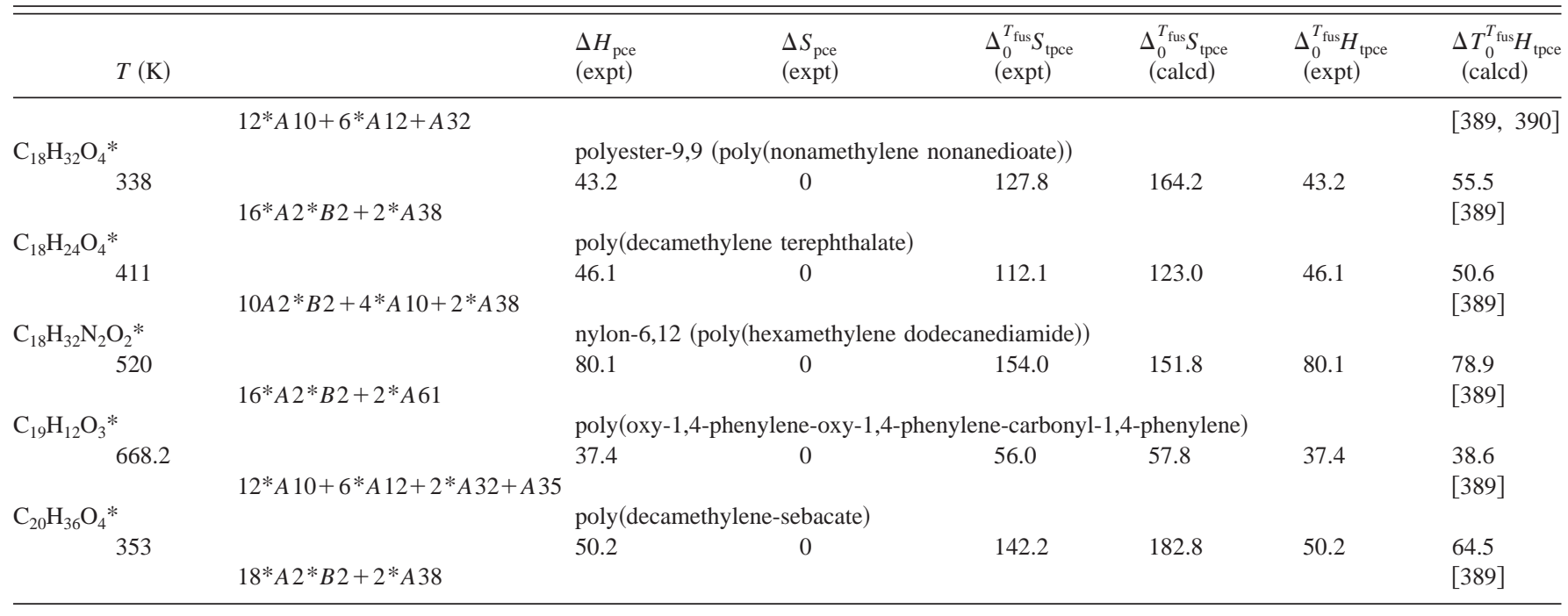

${ }^{\text {a }}$ These compounds were not included in generating the statistics. 
TABLE 7. Calculated and experimental phase change enthalpies and entropies of test solids ${ }^{\mathrm{a}}$

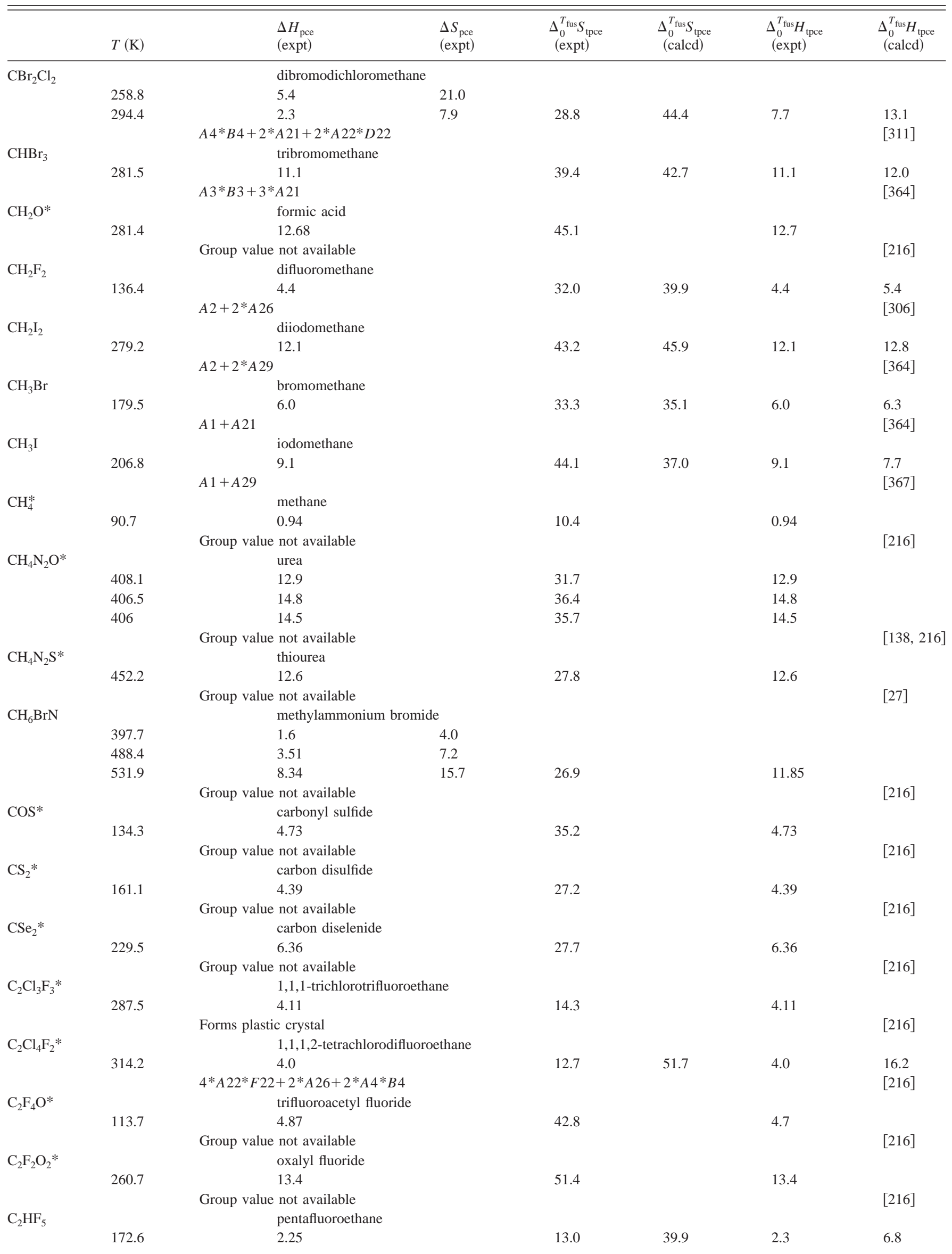


TABLE 7. Calculated and experimental phase change enthalpies and entropies of test solids-Continued

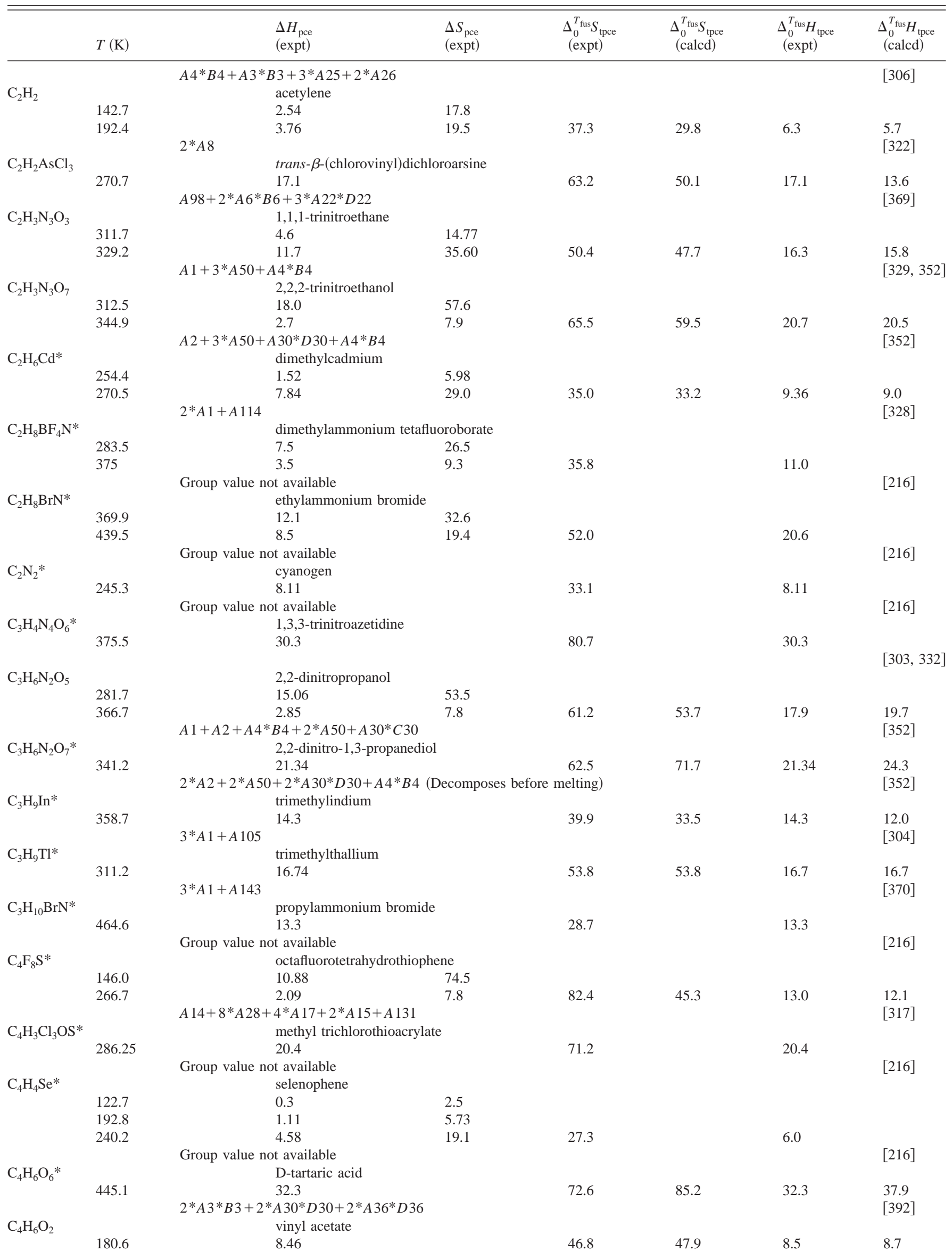


TABLE 7. Calculated and experimental phase change enthalpies and entropies of test solids-Continued

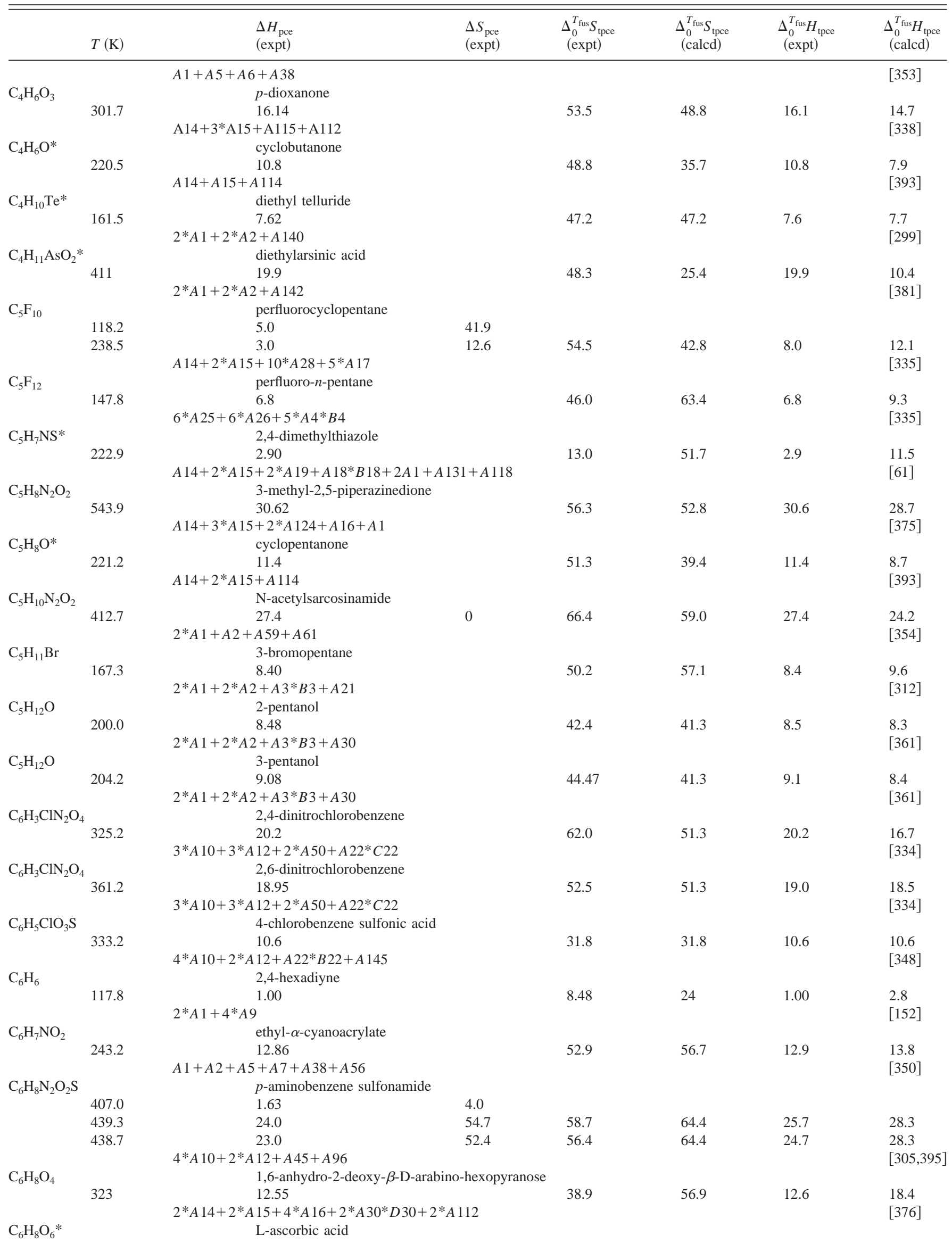


TABLE 7. Calculated and experimental phase change enthalpies and entropies of test solids-Continued

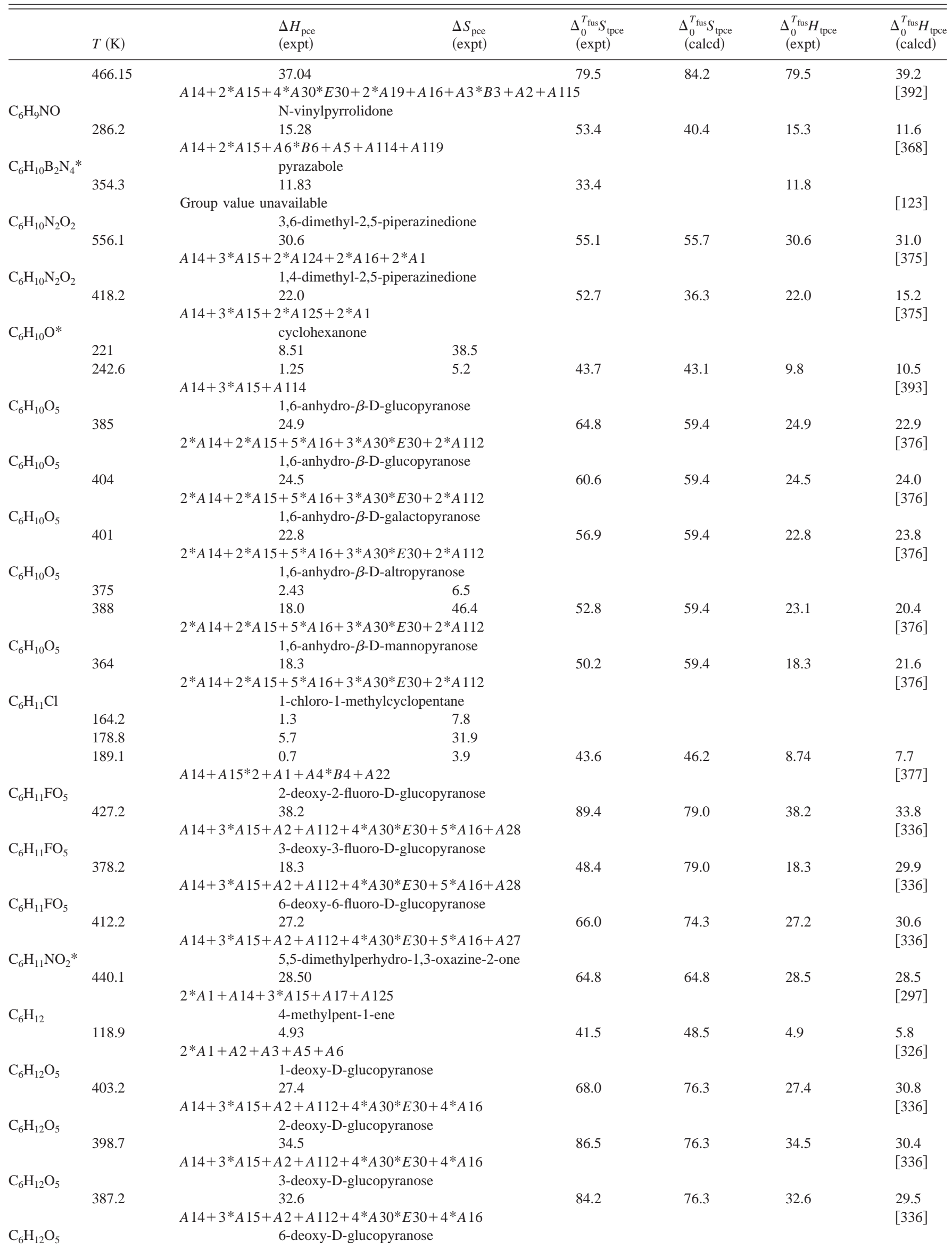


TABLE 7. Calculated and experimental phase change enthalpies and entropies of test solids-Continued

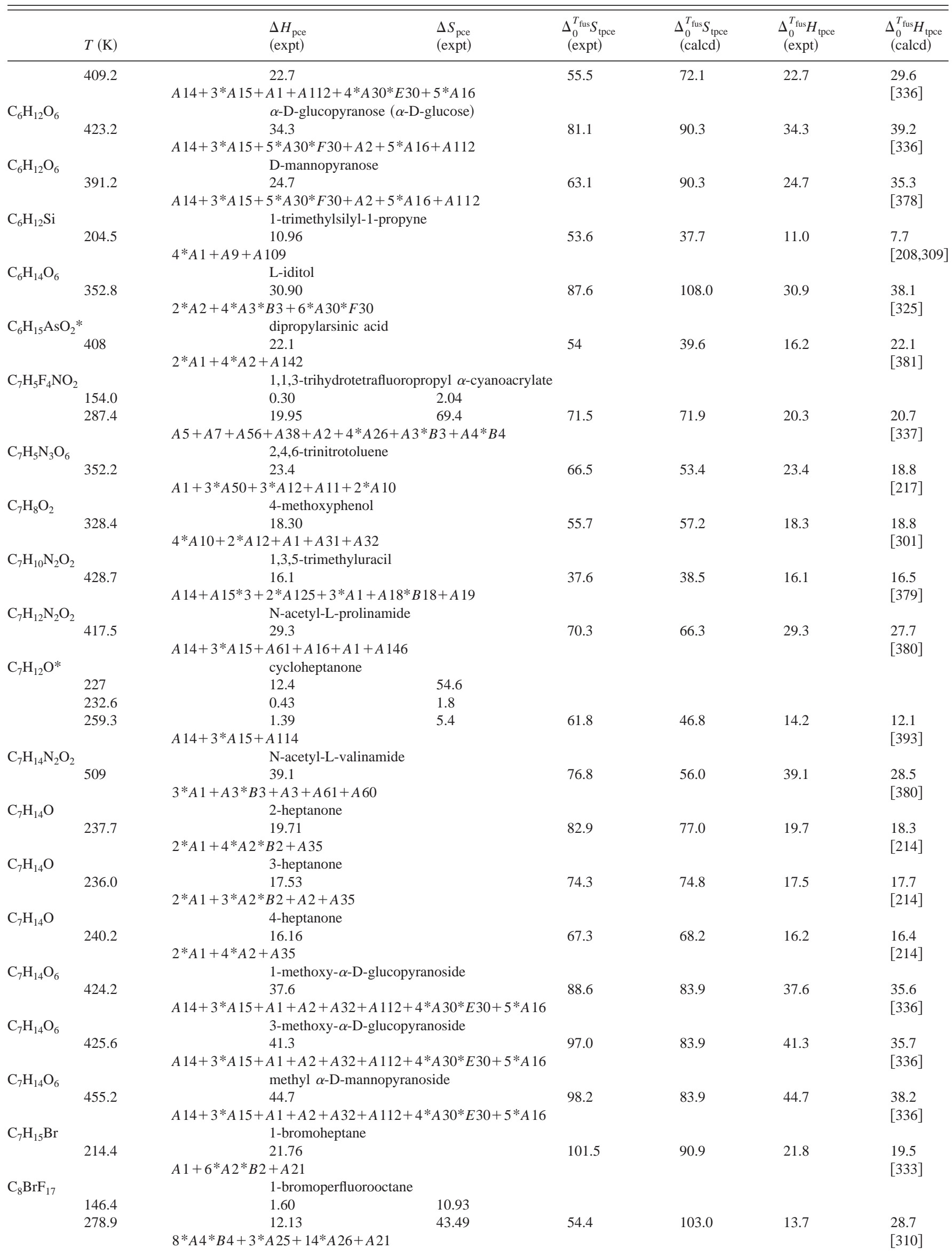


TABLE 7. Calculated and experimental phase change enthalpies and entropies of test solids-Continued

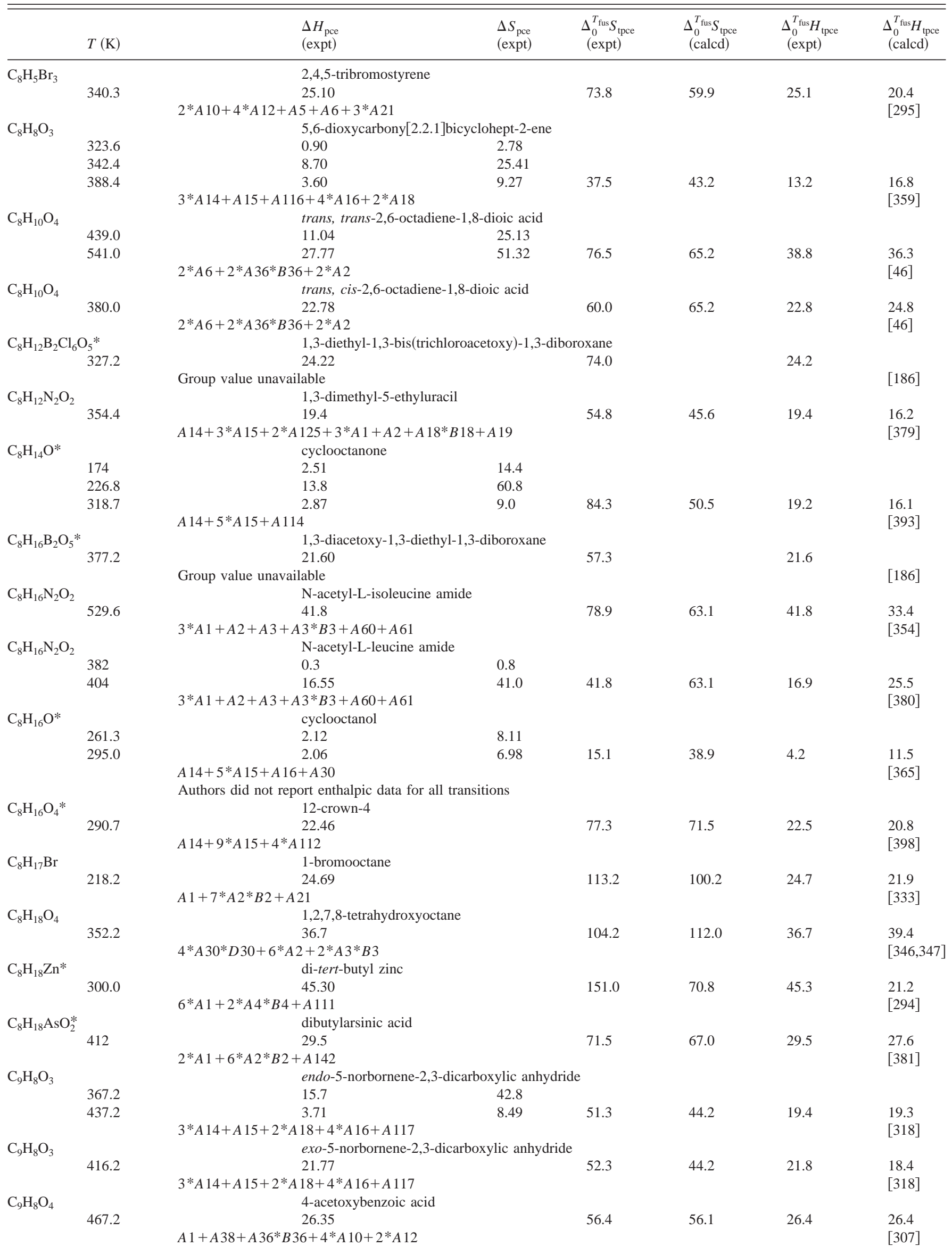


TABLE 7. Calculated and experimental phase change enthalpies and entropies of test solids-Continued

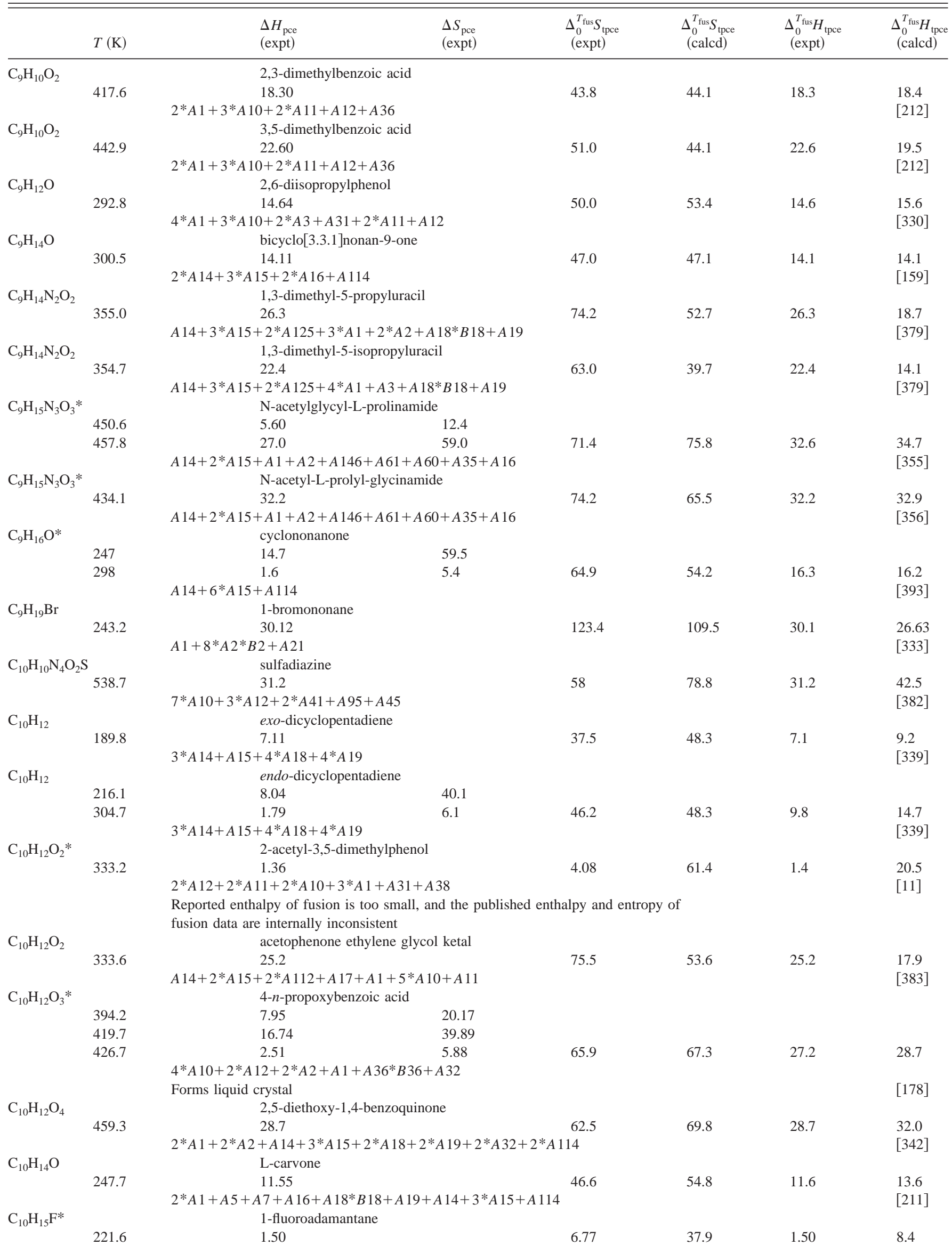


TABLE 7. Calculated and experimental phase change enthalpies and entropies of test solids-Continued

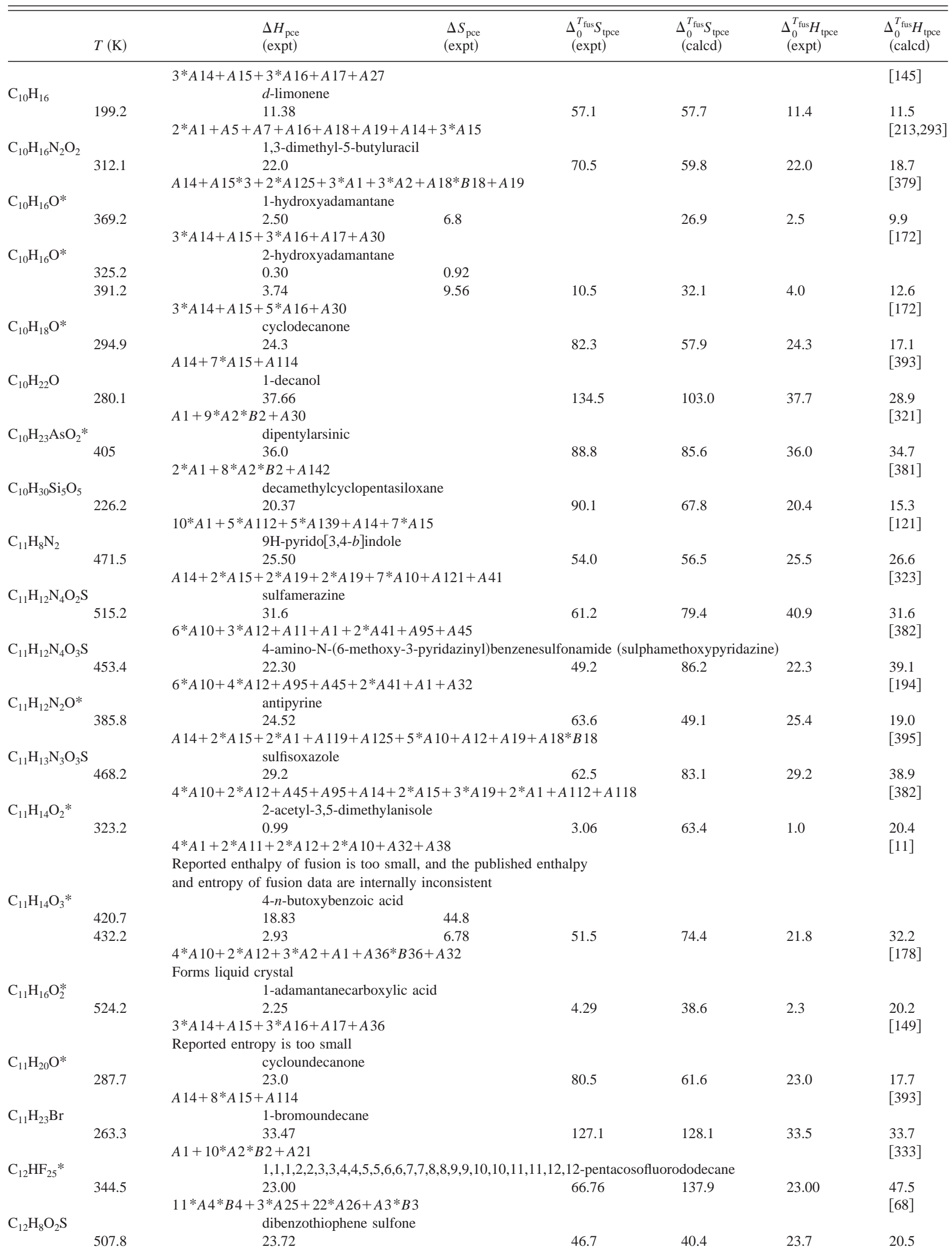


TABLE 7. Calculated and experimental phase change enthalpies and entropies of test solids-Continued

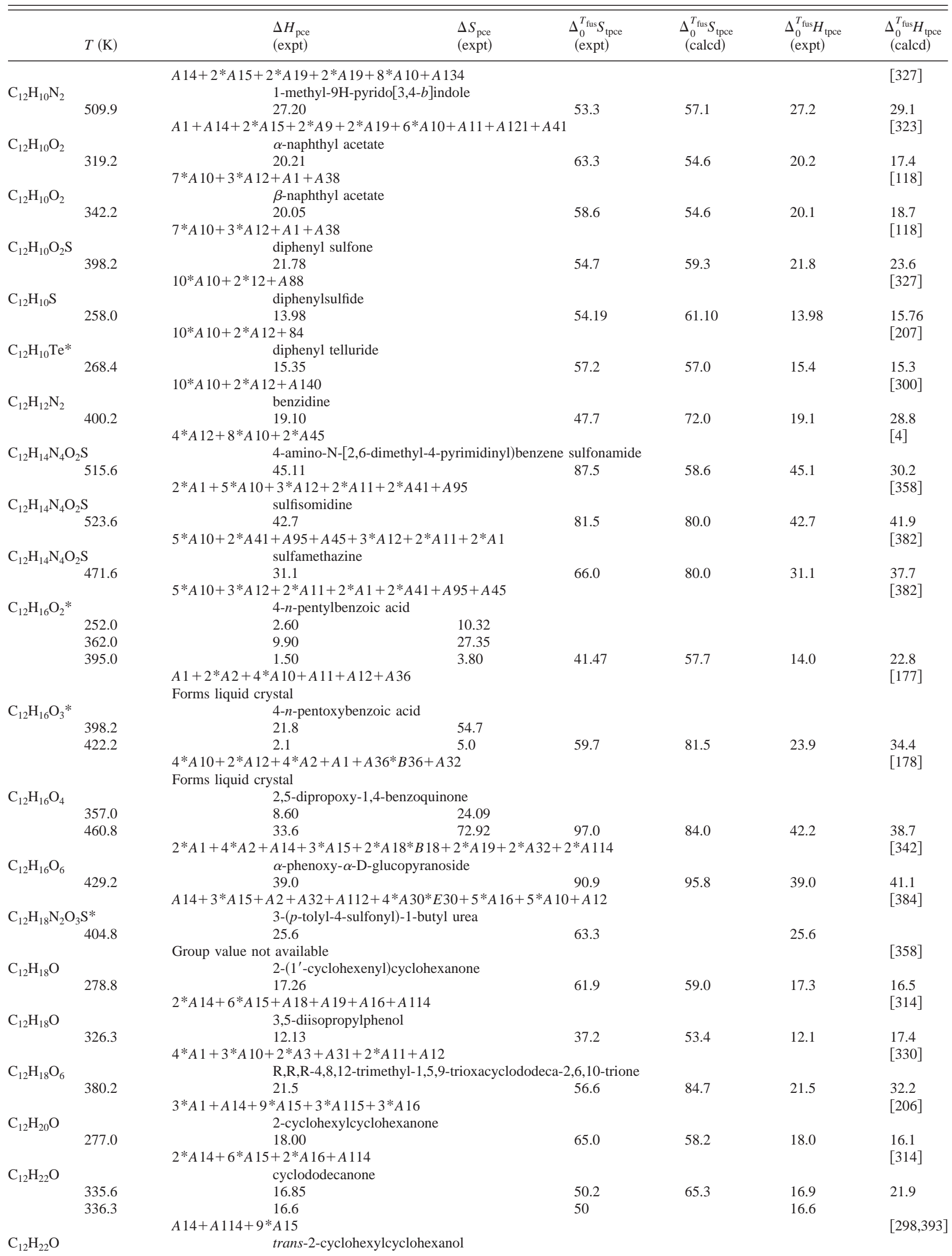


TABLE 7. Calculated and experimental phase change enthalpies and entropies of test solids-Continued

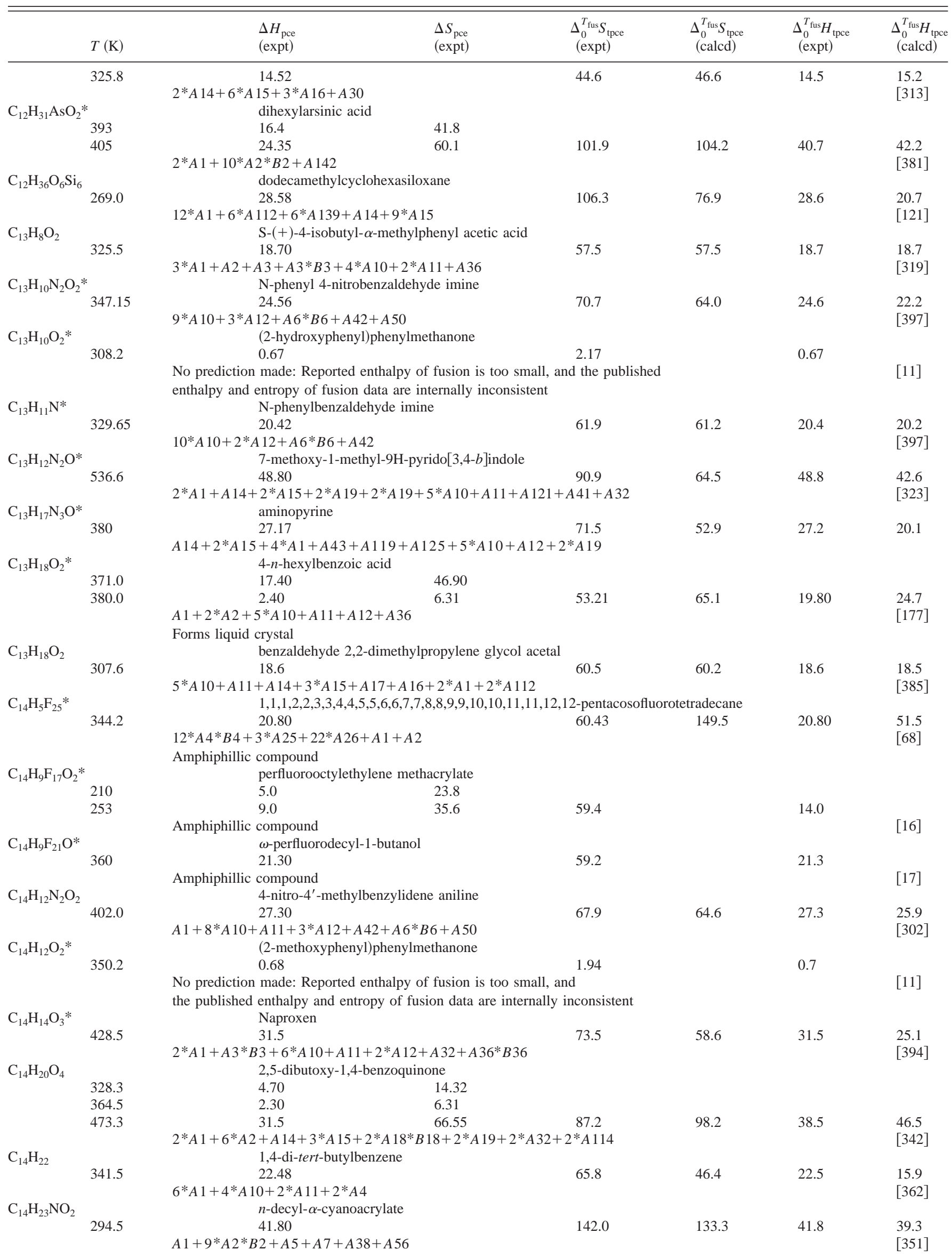


TABLE 7. Calculated and experimental phase change enthalpies and entropies of test solids-Continued

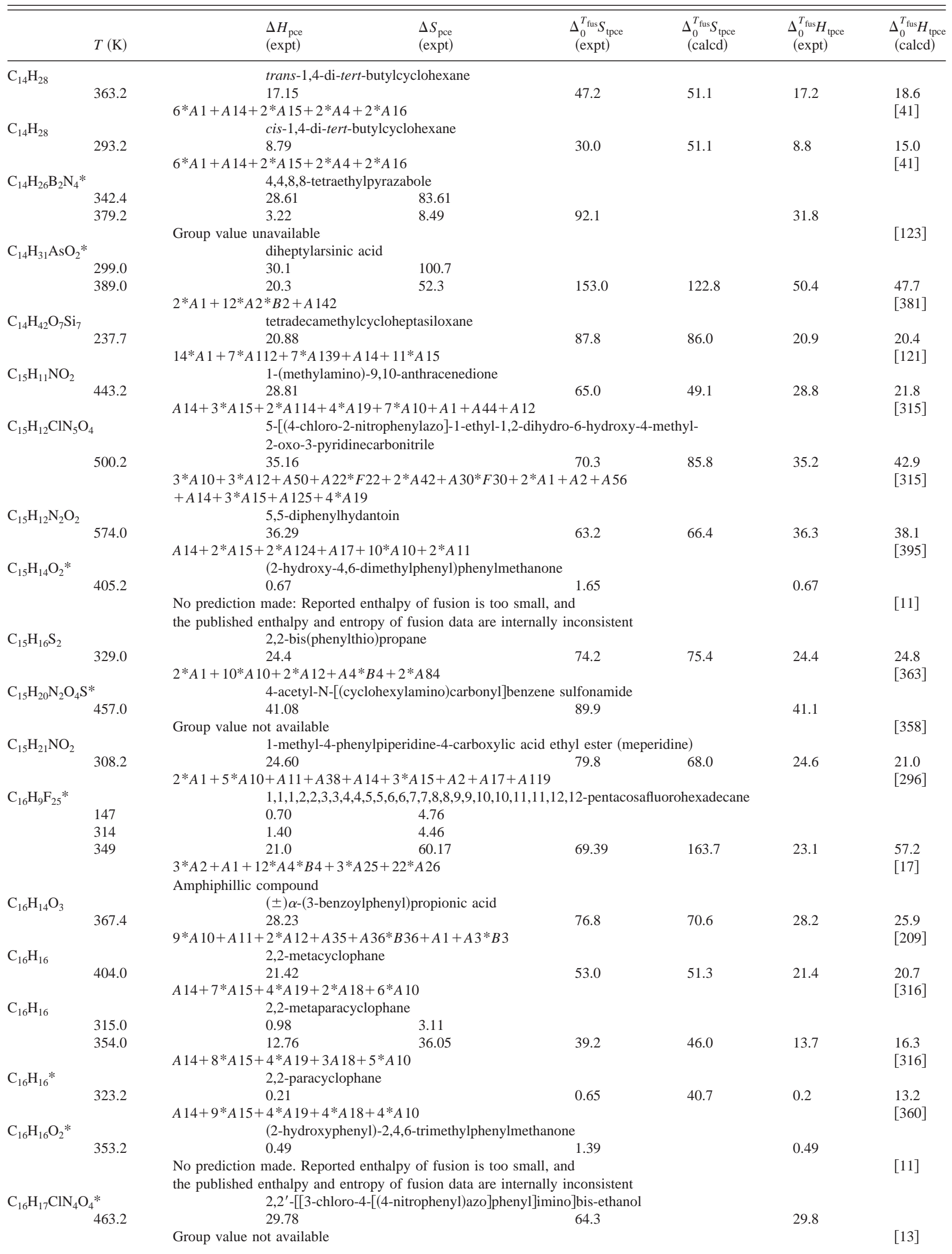


TABLE 7. Calculated and experimental phase change enthalpies and entropies of test solids-Continued

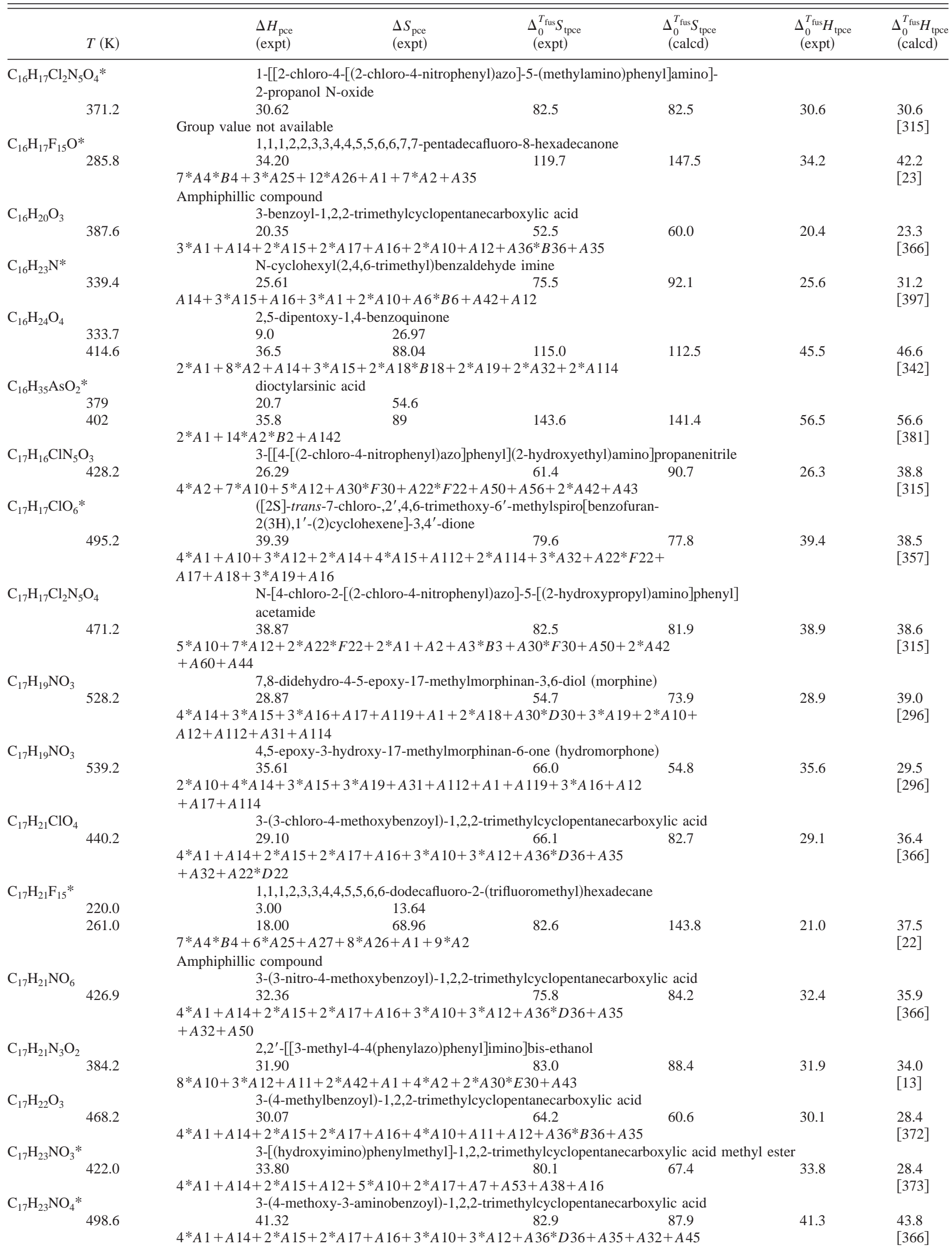


TABLE 7. Calculated and experimental phase change enthalpies and entropies of test solids-Continued

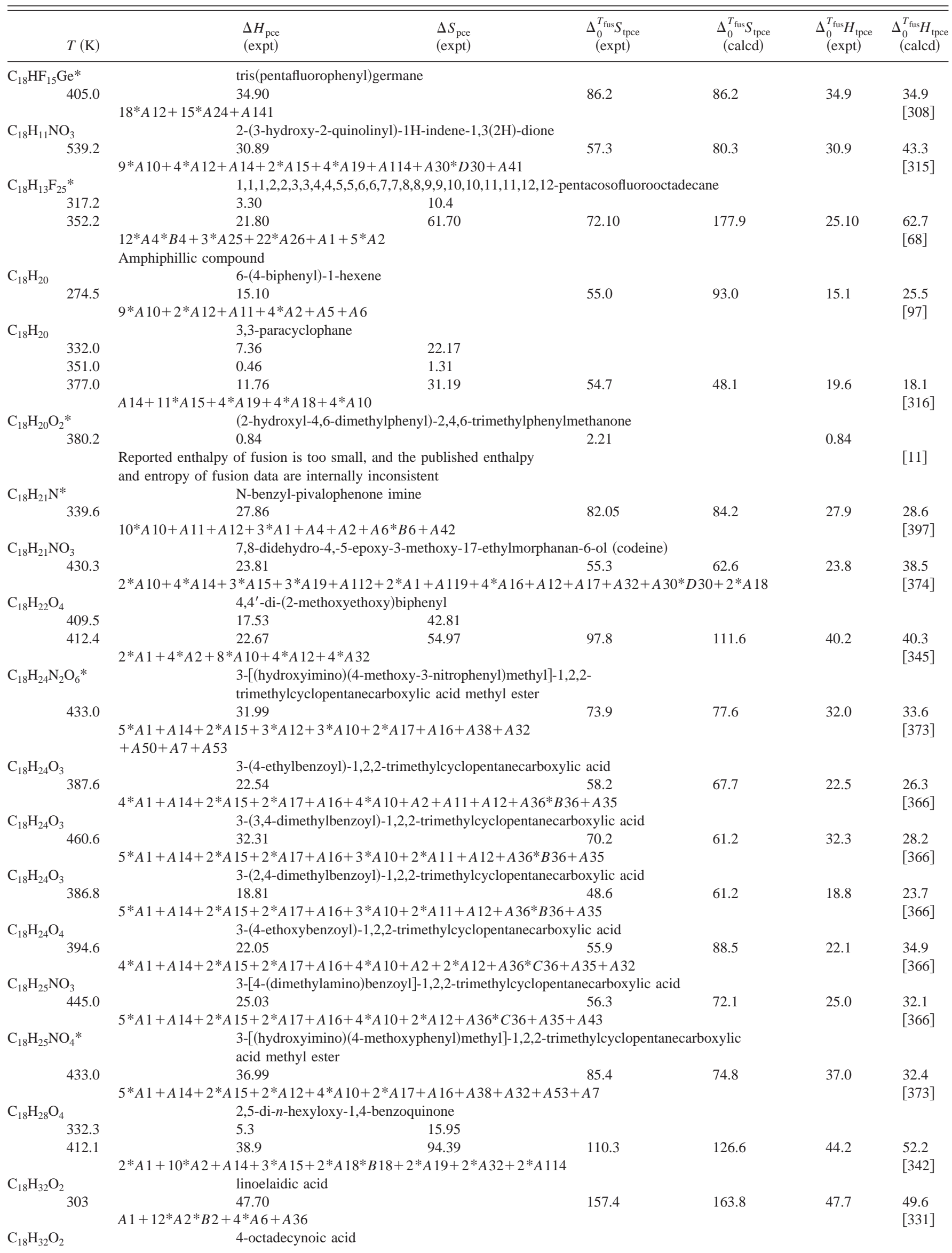


TABLE 7. Calculated and experimental phase change enthalpies and entropies of test solids-Continued

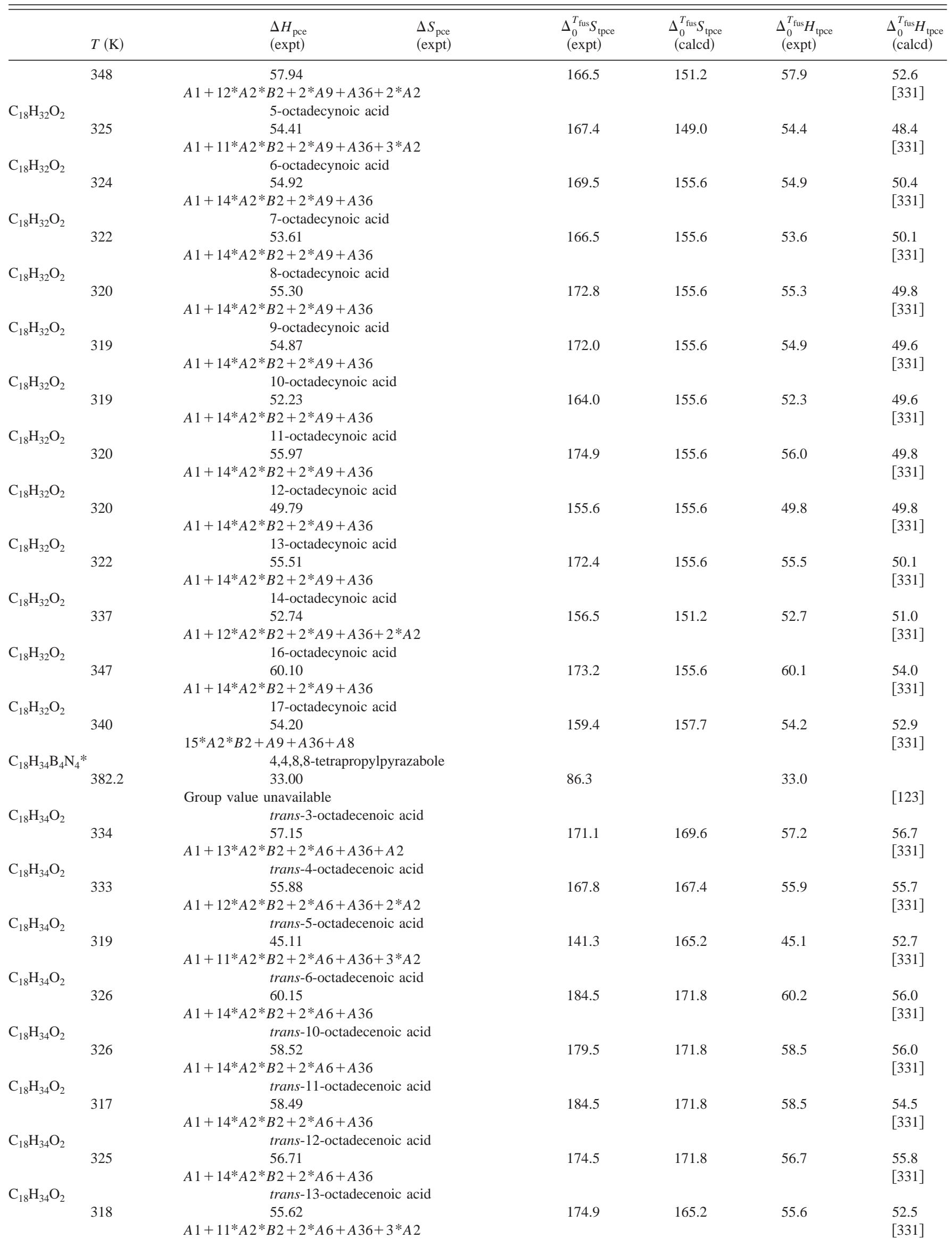


TABLE 7. Calculated and experimental phase change enthalpies and entropies of test solids-Continued

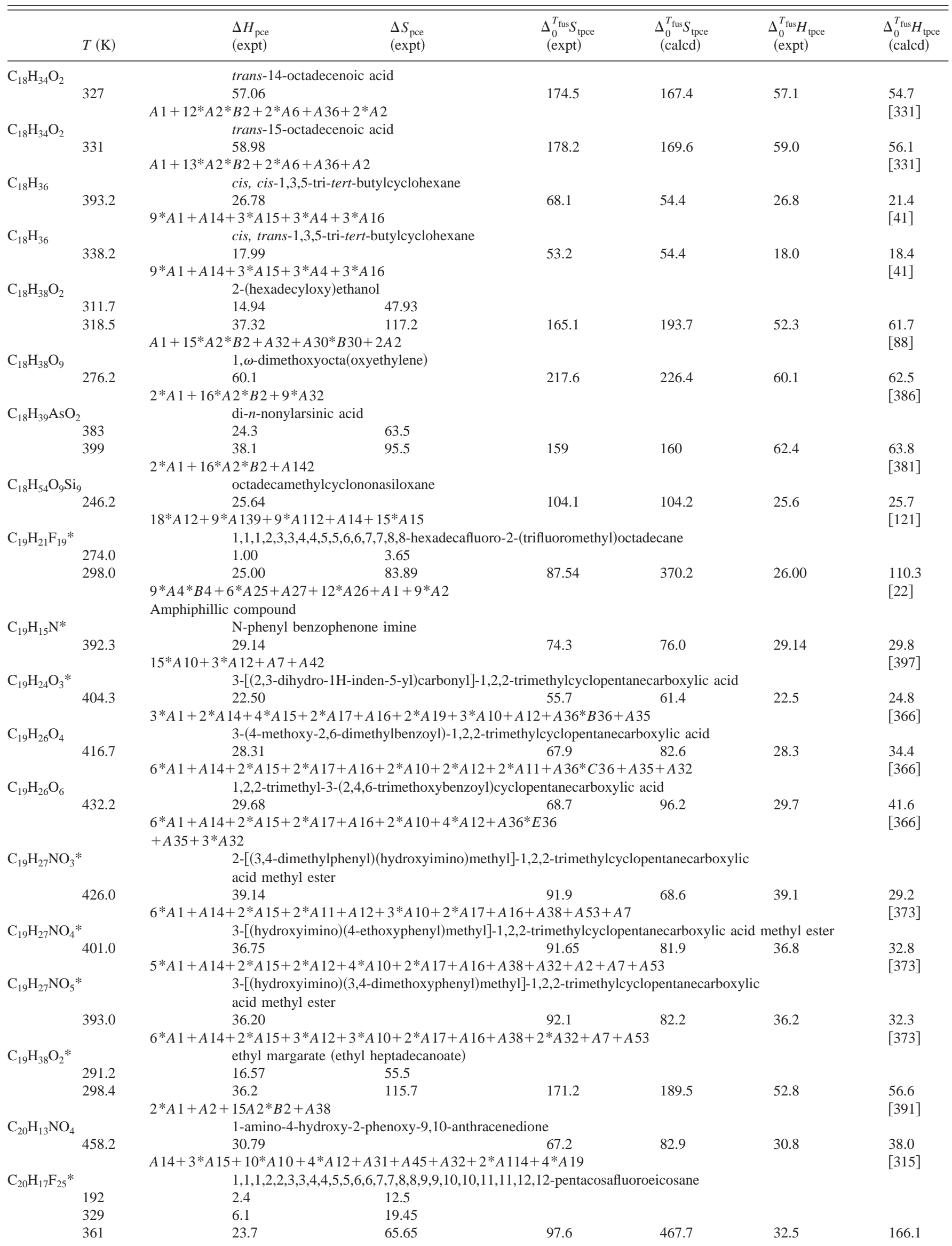


TABLE 7. Calculated and experimental phase change enthalpies and entropies of test solids-Continued

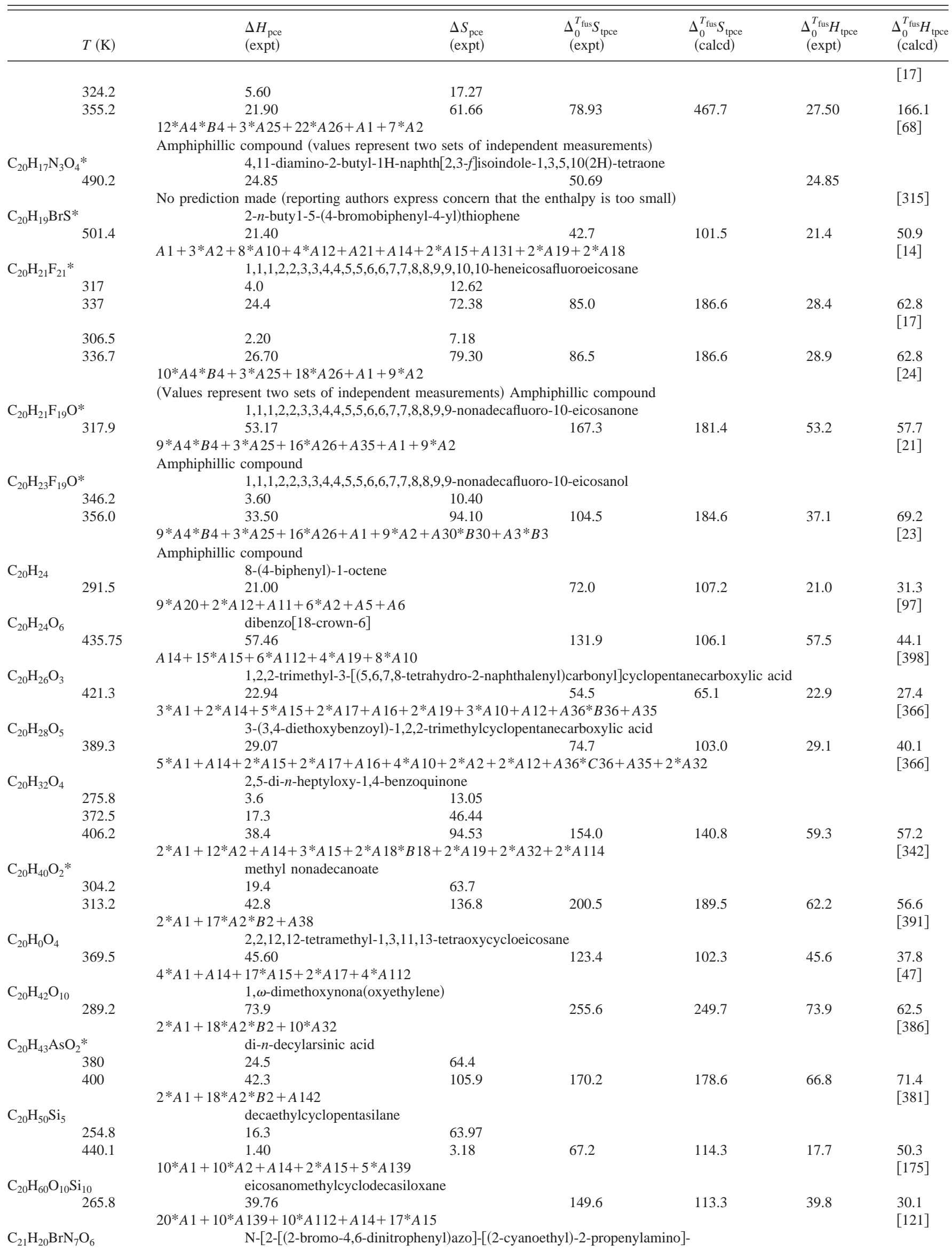


TABLE 7. Calculated and experimental phase change enthalpies and entropies of test solids-Continued

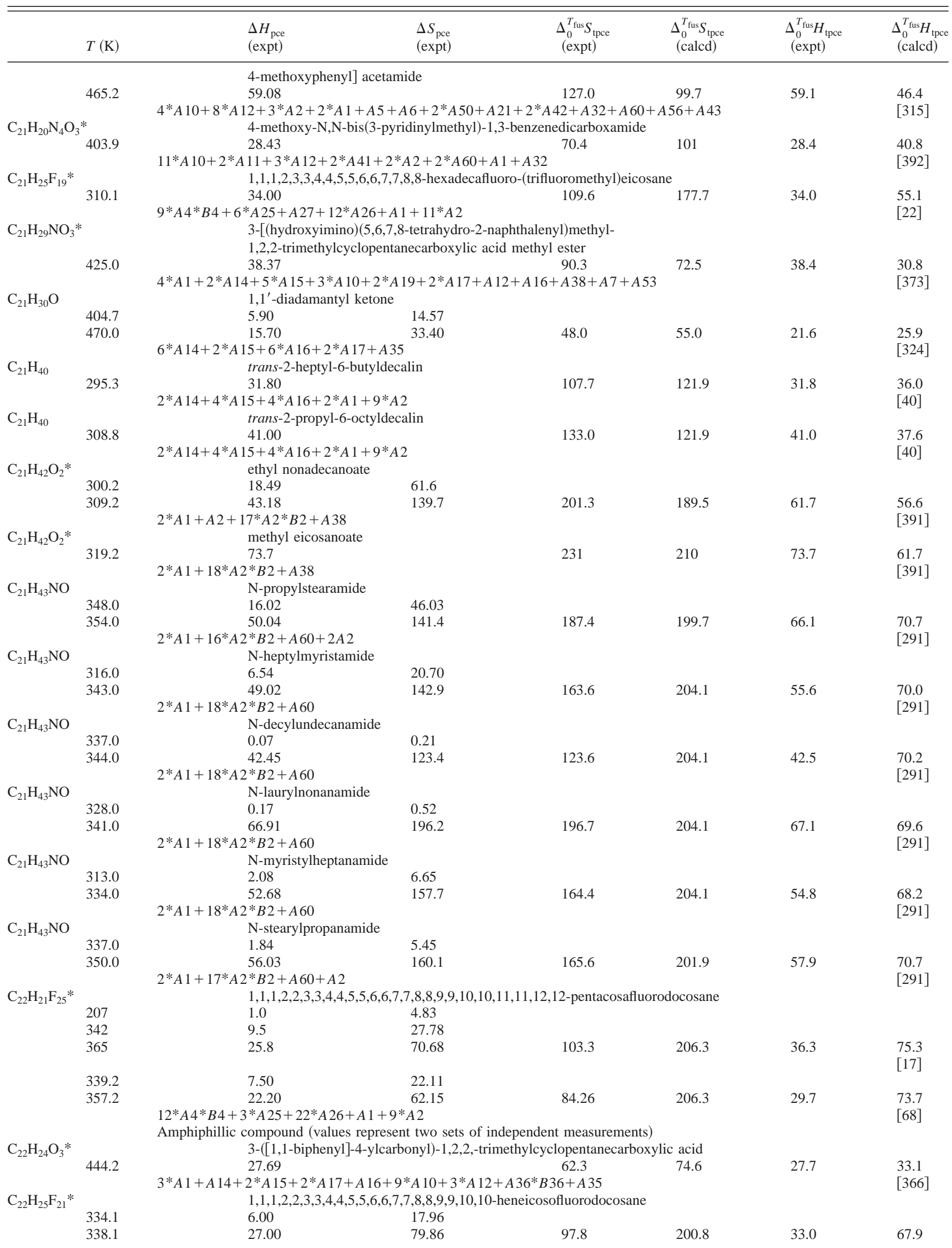


TABLE 7. Calculated and experimental phase change enthalpies and entropies of test solids-Continued

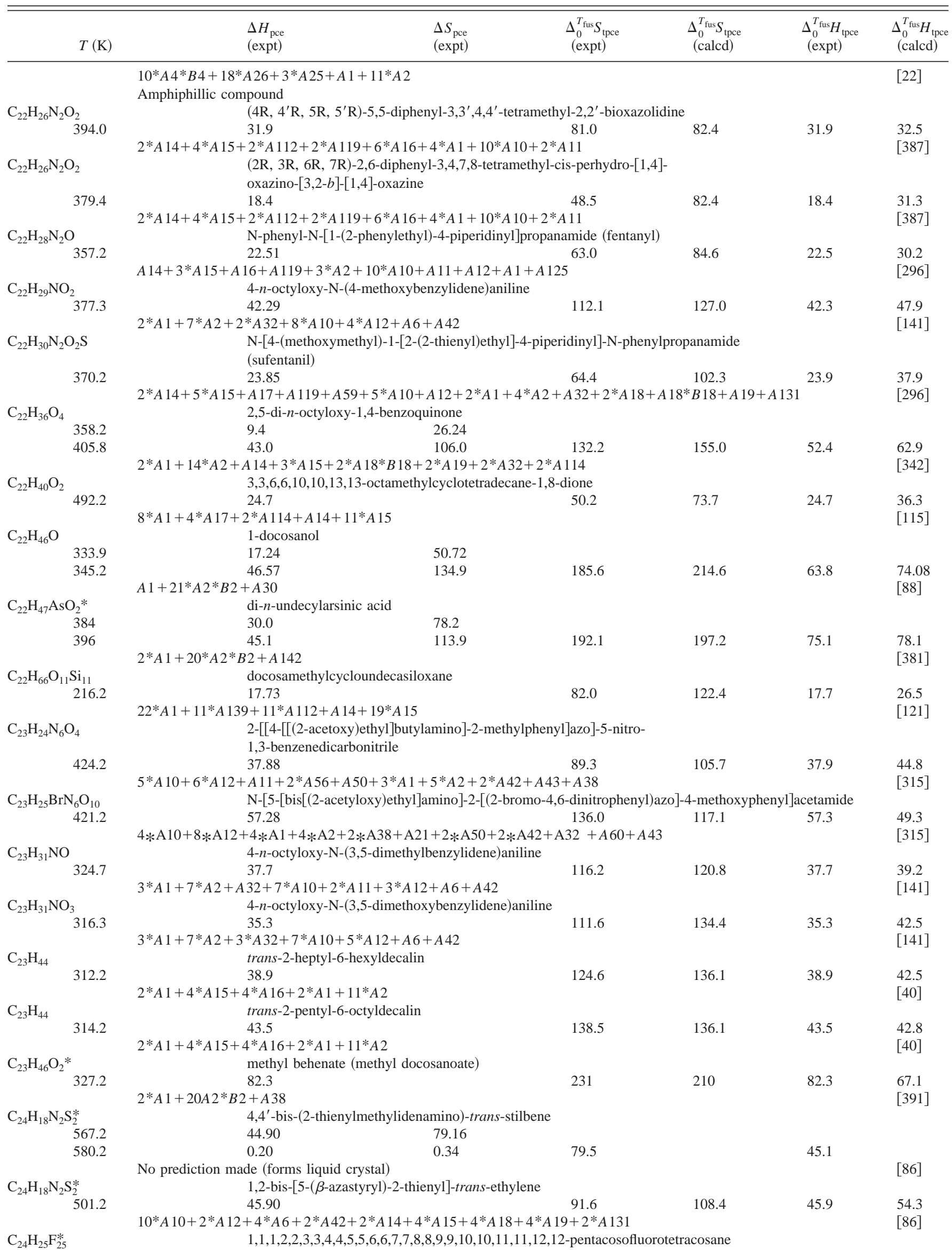


TABLE 7. Calculated and experimental phase change enthalpies and entropies of test solids-Continued

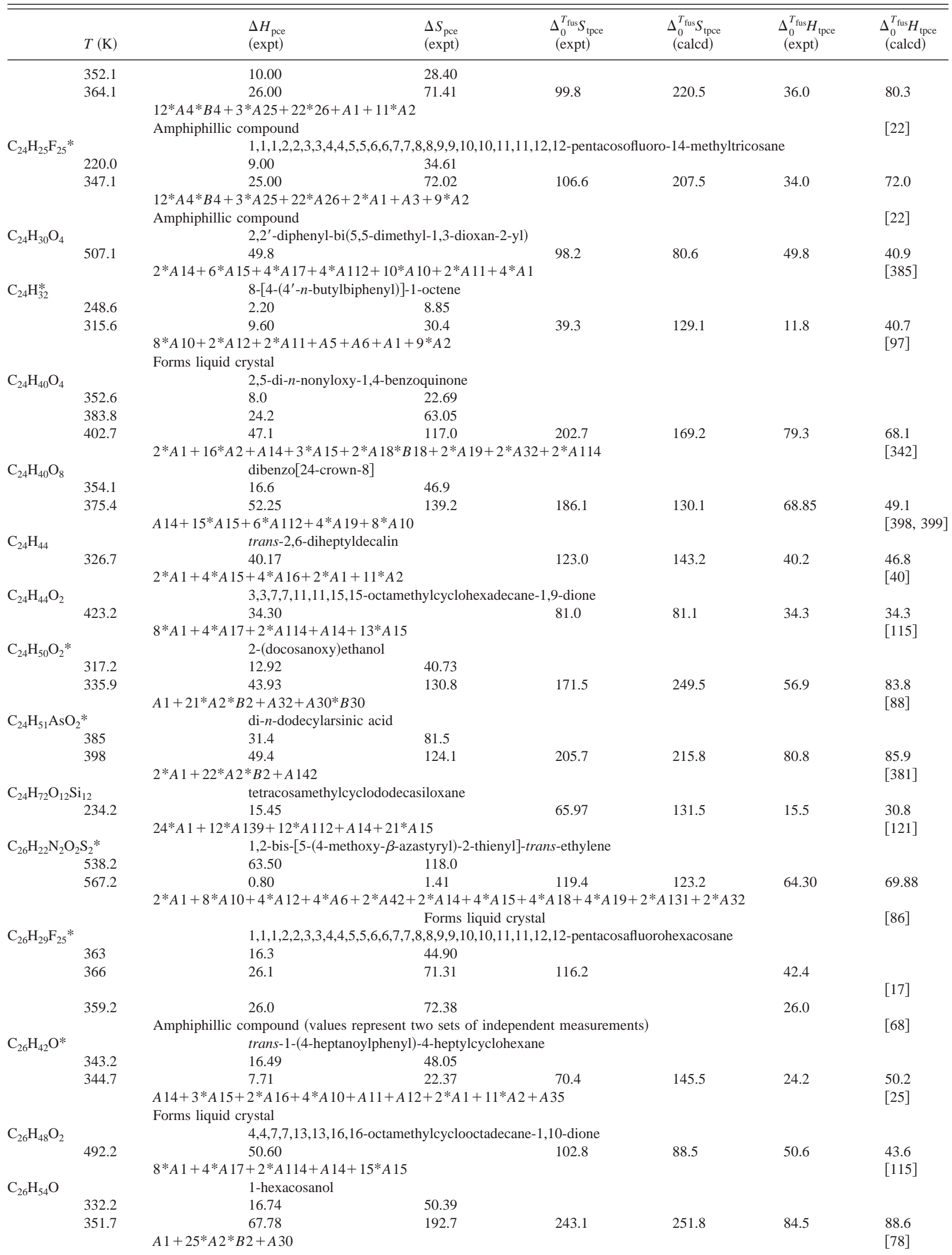


TABLE 7. Calculated and experimental phase change enthalpies and entropies of test solids-Continued

\begin{tabular}{|c|c|c|c|c|c|c|c|}
\hline & $T(\mathrm{~K})$ & $\begin{array}{l}\Delta H_{\text {pce }} \\
(\text { expt })\end{array}$ & $\begin{array}{l}\Delta S_{\text {pce }} \\
(\text { expt })\end{array}$ & $\begin{array}{c}\Delta_{0}^{T_{\text {fus }}} S_{\text {tpce }} \\
(\text { expt) }\end{array}$ & $\begin{array}{c}\Delta_{0}^{T_{\text {fus }}} S_{\text {tpce }} \\
\text { (calcd) }\end{array}$ & $\begin{array}{c}\Delta_{0}^{T_{\text {fus }}} H_{\text {tpce }} \\
(\text { expt })\end{array}$ & $\begin{array}{c}\Delta_{0}^{T_{\text {fus }}} H_{\text {tpce }} \\
\text { (calcd) }\end{array}$ \\
\hline \multirow[t]{4}{*}{$\mathrm{C}_{26} \mathrm{H}_{55} \mathrm{AsO}_{2}{ }^{*}$} & & di- $n$-tric & & & & & \\
\hline & 388 & 36.5 & 94.0 & & & & \\
\hline & 396 & 52.7 & 133.1 & 227.2 & 234.4 & 89.2 & 92.8 \\
\hline & & \multicolumn{5}{|c|}{$2 * A 1+24 * A 2 * B 2+A 142$} & [381] \\
\hline \multirow{2}{*}{\multicolumn{2}{|c|}{$\begin{array}{r}\mathrm{C}_{27} \mathrm{H}_{42} \mathrm{Cl}_{2} \mathrm{~N}_{2} \mathrm{O}_{6} * \\
367.3\end{array}$}} & & polymo & & & & \\
\hline & & $\begin{array}{l}51.04 \\
\text { chloram }\end{array}$ & $\begin{array}{l}0 \\
\text { polymor }\end{array}$ & 139 & 188.6 & 51.04 & 69.2 \\
\hline & 360.8 & 41.3 & 0 & 112.5 & 188.6 & 41.3 & 69.2 \\
\hline & & \multicolumn{5}{|c|}{$\begin{array}{l}4^{*} A 10+A 11+A 12+A 50+A 30^{*} F 30+2 * A 22 * F 22+A 60+A 38+A 2+3 * A 3 * B 3+ \\
A 1+14^{*} A 2\end{array}$} & [395] \\
\hline \multirow{3}{*}{$\mathrm{C}_{28} \mathrm{H}_{22} \mathrm{~N}_{2} \mathrm{O}_{2}$} & \multicolumn{6}{|c|}{ 1,4-[bis[(4-methylphenyl)amino]-9,10-anthracenedione } & \\
\hline & 491.2 & 36.59 & & 74.5 & 71.5 & 36.6 & 35.1 \\
\hline & \multirow{2}{*}{\multicolumn{7}{|c|}{$1,1,1,2,2,3,3,4,4,5,5,6,6,7,7,8,8,9,9,10,10,11,11,12,12$-pentacosofluorooctacosane }} \\
\hline \multirow{4}{*}{$\mathrm{C}_{28} \mathrm{H}_{31} \mathrm{~F}_{25} *$} & & & & & & & \\
\hline & 263.2 & 43.10 & & 163.8 & 248.9 & 43.10 & 65.5 \\
\hline & & \multicolumn{5}{|c|}{$12 * A 4 * B 4+3 * A 25+22 * A 26+A 1+15 * A 2$} & [68] \\
\hline & & \multicolumn{6}{|c|}{ Amphiphillic compound } \\
\hline \multirow[t]{5}{*}{$\mathrm{C}_{28} \mathrm{H}_{48} \mathrm{O}^{*}$} & \multirow{2}{*}{\multicolumn{6}{|c|}{ trans-1-heptyl-4-(4-nonanoylphenyl)cyclohexane }} & \\
\hline & 343.4 & 20.8 & & & & & \\
\hline & 353.3 & 11.32 & 32.1 & 92.6 & 159.7 & 32.1 & 56.4 \\
\hline & & $A 14+3 * A 15+2 * A 1$ & $A 12+2$ & $+A 35$ & & & \\
\hline & & Forms liquid crystal & & & & & [25] \\
\hline $\mathrm{C}_{28} \mathrm{H}_{48} \mathrm{O}_{4}$ & & $2,5-\mathrm{di}-n$ & nzoquin & & & & \\
\hline & 367.4 & 12.9 & 35.11 & & & & \\
\hline & 390.0 & 28.4 & 72.8 & & & & \\
\hline & 397.2 & 52.1 & 131.2 & 239.1 & 241.6 & 93.4 & 96.0 \\
\hline & & $2 * A 1+20 * A 2 * B 2+$ & $A 18^{*} B 1$ & $2 * A 32+2$ & & & [342] \\
\hline $\mathrm{C}_{28} \mathrm{H}_{52} \mathrm{O}_{2}$ & & $4,4,8,8$ & thylcycl & 1-dione & & & \\
\hline & 418.2 & 36.80 & & 88.0 & 95.9 & 36.8 & 40.1 \\
\hline & & $8 * A 1+4 * A 17+2 *$ & 15 & & & & {$[115]$} \\
\hline $\mathrm{C}_{28} \mathrm{H}_{59} \mathrm{AsO}_{2}{ }^{*}$ & & di- $n$-tet & & & & & \\
\hline & 390 & 39.3 & 100.6 & & & & \\
\hline & 397 & 58.2 & 146.6 & 247.2 & 253.0 & 97.5 & 100.5 \\
\hline & & $2 * A 1+26^{*} A 2 * B 2+$ & & & & & {$[381]$} \\
\hline $\mathrm{C}_{29} \mathrm{H}_{41} \mathrm{NO}_{4}$ & & $\begin{array}{l}\text { 17-(cyc } \\
6 \text {-metho }\end{array}$ & $\begin{array}{l}(1,1-\operatorname{dim} \\
\text { ethenom }\end{array}$ & $\begin{array}{l}\text {,5-epoxy-18 } \\
\text { hethanol }\end{array}$ & dro- & & \\
\hline & 491.3 & 26.80 & & 54.55 & 68.6 & 26.80 & 33.7 \\
\hline & & $\begin{array}{l}6^{*} A 14+2 * A 15+5 * \\
3 * A 19+A 12+2 * A 1\end{array}$ & $E 30+4$ & $17+A 119+$ & $31+A 32+$ & & [320] \\
\hline $\mathrm{C}_{30} \mathrm{H}_{37} \mathrm{~F}_{25} *$ & & $1,1,1,2$ & $, 8,8,9,9$, & 12,12-penta & otricontane & & \\
\hline & 365.2 & 47.80 & & 130.9 & 263.1 & 47.80 & 96.1 \\
\hline & & $12 * A 4 * B 4+3 * A 25$ & $7^{*} A 2$ & & & & [68] \\
\hline $\mathrm{C}_{30} \mathrm{H}_{56} \mathrm{O}_{2}$ & & $5,5,8,8$ & thylcycl & 12-dione & & & \\
\hline & 442.2 & 47.70 & & 107.9 & 95.9 & 47.7 & 42.4 \\
\hline & & $8 * A 1+4 * A 17+2 *$ & & & & & {$[115]$} \\
\hline $\mathrm{C}_{30} \mathrm{H}_{60} \mathrm{O}_{15}$ & & 45-crow & & & & & \\
\hline & 311.2 & 70.6 & & 227 & 206.8 & 70.6 & 64.3 \\
\hline & & $A 14+42 * A 15+15 *$ & & & & & {$[386]$} \\
\hline $\mathrm{C}_{30} \mathrm{H}_{63} \mathrm{AsO}_{2}{ }^{*}$ & & di- $n$-per & & & & & \\
\hline & 390 & $46.4^{1}$ & 119 & & & & \\
\hline & 396 & 63.6 & 160.5 & 279.6 & 271.6 & 110.0 & 107.6 \\
\hline & & $2 * A 1+28 * A 2 * B 2+$ & & & & & {$[381]$} \\
\hline $\mathrm{C}_{31} \mathrm{H}_{43} \mathrm{NO}_{5}$ & & $\begin{array}{l}\text { 3-(acety } \\
18,19-d\end{array}$ & $\begin{array}{l}\text { pylmeth } \\
\alpha \text {-methyl }\end{array}$ & $\begin{array}{l}\text { nethylethyl } \\
\text { morphinan- }\end{array}$ & & & \\
\hline & 440.3 & 22.40 & & 50.9 & 68.9 & 22.4 & 30.3 \\
\hline & & $\begin{array}{l}6 * A 14+2 * A 15+6 * \\
3 * A 19+A 12+2 * A 1\end{array}$ & $E 30+4$ & $17+A 119+$ & & & [320] \\
\hline $\mathrm{C}_{32} \mathrm{H}_{34}$ & & 1,8-bis- & & & & & \\
\hline & 415.2 & 56.00 & & 134.9 & 140.8 & 56.0 & 58.5 \\
\hline & & $18 * A 10+4 * A 12+2$ & & & & & [97] \\
\hline $\mathrm{C}_{32} \mathrm{H}_{34}{ }^{*}$ & & 1,8-bis [ & Jbutane & & & & \\
\hline & 454.2 & 46.00 & & 101.3 & 127.8 & 46.0 & 58.1 \\
\hline & & $2 * A 1+6 * A 2+16^{*}$ & & & & & [97] \\
\hline $\mathrm{C}_{32} \mathrm{H}_{41} \mathrm{~F}_{25} *$ & & $1,1,1,2$ & $8,8,9,9$, & 12,12-penta & odotriaconta & & \\
\hline & 369.2 & 43.40 & & 117.6 & 277.3 & 43.4 & 102.3 \\
\hline & & $12 * A 4 * B 4+3 * A 25$ & $9 * A 2$ & & & & [68] \\
\hline $\mathrm{C}_{32} \mathrm{H}_{64} 16$ & & 48-crov & & & & & \\
\hline & 312.2 & 59.1 & & 189.4 & 219.1 & 59.1 & 68.4 \\
\hline & & $A 14+45^{*} A 15+16^{*}$ & & & & & {$[386]$} \\
\hline
\end{tabular}


TABLE 7. Calculated and experimental phase change enthalpies and entropies of test solids-Continued

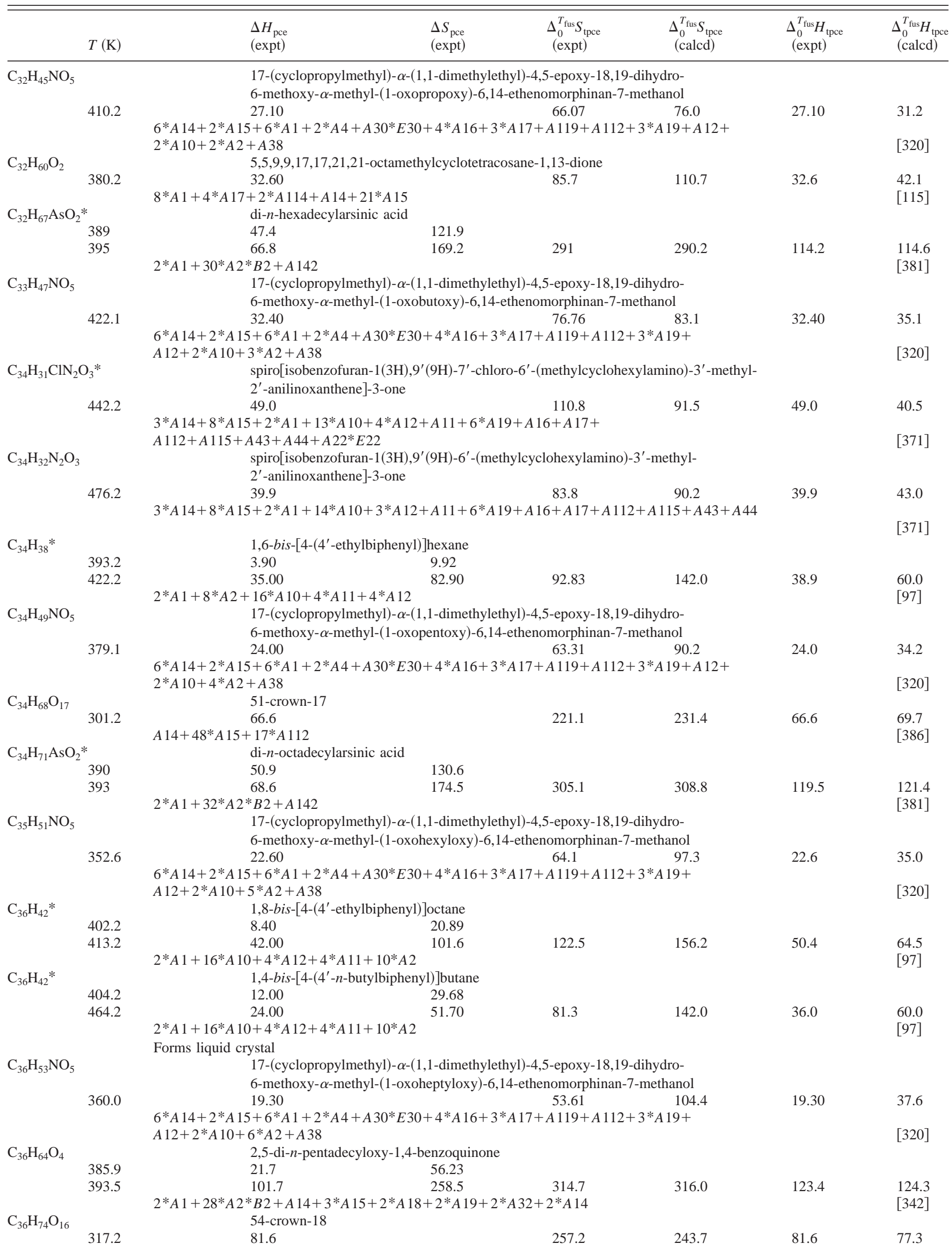


TABLE 7. Calculated and experimental phase change enthalpies and entropies of test solids-Continued

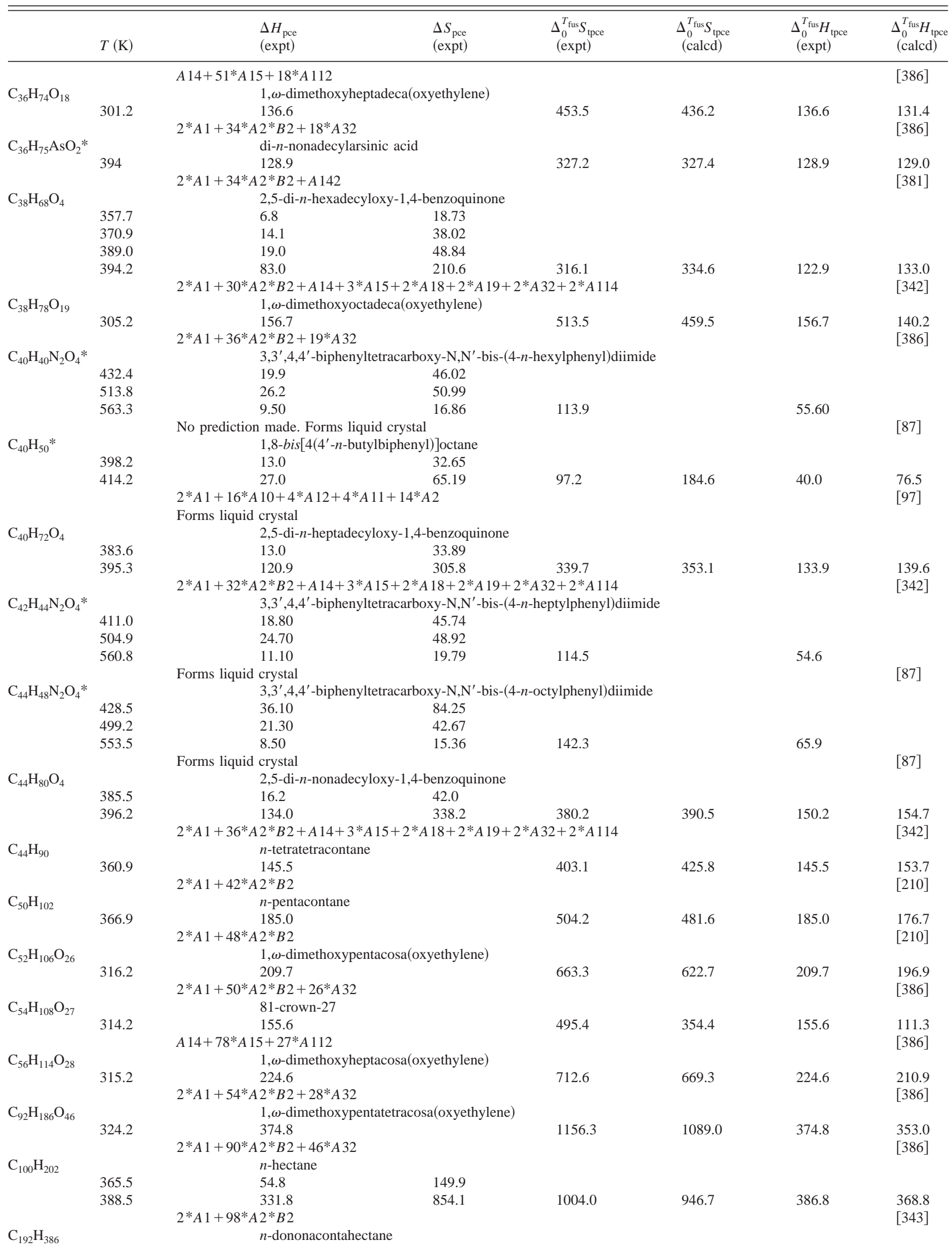


PHASE CHANGE ENTHALPIES AND ENTROPIES

TABLE 7. Calculated and experimental phase change enthalpies and entropies of test solids-Continued

\begin{tabular}{|c|c|c|c|c|c|c|}
\hline$T(\mathrm{~K})$ & $\begin{array}{l}\Delta H_{\text {pce }} \\
(\text { expt) }\end{array}$ & $\begin{array}{l}\Delta S_{\text {pce }} \\
(\text { expt) }\end{array}$ & $\begin{array}{l}\Delta_{0}^{T_{\text {fus }}} S_{\text {tpce }} \\
(\text { expt })\end{array}$ & $\begin{array}{l}\Delta_{0}^{T_{\text {fus }}} S_{\text {tpce }} \\
(\text { calcd })\end{array}$ & $\begin{array}{l}\Delta_{0}^{T_{\text {fus }}} H_{\text {tpce }} \\
(\text { expt })\end{array}$ & $\begin{array}{l}\Delta_{0}^{T_{\text {fus }}} H_{\text {tpce }} \\
\text { (calcd) }\end{array}$ \\
\hline 399.1 & $\begin{array}{r}698.9 \\
2 * A 1+190 * A 2 * B 2\end{array}$ & a smal & $\begin{array}{l}1751.2 \\
\text { transition) }\end{array}$ & 1802.4 & 698.9 & $\begin{array}{l}719.3 \\
{[344]}\end{array}$ \\
\hline
\end{tabular}

${ }^{\mathrm{a} U n i t s ~ f o r ~} \Delta_{0}^{T_{\text {fus }}} S_{\text {tpce }}$ and $\Delta_{0}^{T_{\text {fus }}} H_{\text {tpce }}$ are $\mathrm{J} \cdot \mathrm{mol}^{-1} \cdot \mathrm{K}^{-1}$ and $\mathrm{kJ} \cdot \mathrm{mol}^{-1}$, respectively; compounds with molecular formulas characterized with an asterisk $(*)$ were not included in generating the statistics. As noted in the table, some of these compounds exhibit liquid crystal behavior, others display amphiphillic behavior, group values for some are not currently available, the error between experimental and calculated total phase change entropy exceeded three standard deviations or some may have been added at a later date. 
Table 8. References to Tables 5, 6, and 7

1. N. Okamoto, M. Oguni, and H. Saga, Thermochim. Acta 202, 215 (1992). 2. P. Figuiere, H. Szwarc, M. Oguni, and H. Suga, J. Chem. Thermodyn. 17, 949 (1985)

3. M. Radomska and R. Radomski, J. Thermal Anal. 37, 693 (1991).

4. U. S. Rai and S. George, Can. J. Chem. 70, 2869 (1992).

5. A. A. Kozyro, G. J. Kabo, V. S. Krouk, M. S. Sheiman, I. A. Yursha, V. V. Simirsky, A. P. Krasulin, V. M. Sevruk, and V. I. Gogolinsky, J. Chem. Thermodyn. 24, 883 (1992).

6. G. J. Kabo, A. A. Kozyro, V. S. Krouk, V. M. Sevruk, I. A. Yursha, V. V. Simirsky, and V. I. Gogolinsky, J. Chem. Thermodyn. 24, 1 (1992).

7. Z.-C. Tan and R. Sabbah, Thermochim. Acta 231, 109 (1994).

8. R. Sabbah and T. H. D. Le, Can. J. Chem. 71, 1378 (1993).

9. Z. Z. Liu, X. D. Guo, L. E. Straub, G. Erdos, R. J. Prankerd, R. J. Gozalez-Rothi, and H. Schreier, Drug Design Discovery 8, 57 (1991). 10. M. G. M. Van der Vis, E. H. P. Cordfunke, R. J. M. Konings, G. J. K. Van der Berg, and J. C. Van Miltenburg, J. Chem. Thermodyn. 24, 1103 (1992).

11. S. R. Salman and K. F. Abas, Thermochim. Acta 152, 381 (1989).

12. W. V. Steele, R. D. Chirico, S. E. Knipmeyer, and A. Nguyen J.

Chem. Thermodyn. 25, 965 (1993).

13. G. L. Baughman and T. A. Perenich, Environ. Tox. Chem. 7, 183 (1988).

14. R. Brettle, D. A. Dunmur, C. M. Marson, M. Pinol, and K. Toriyama, Liq. Cryst. 13, 515 (1993)

15. W. V. Steele, R. D. Chirico, S. E. Knipmeyer, and A. Nguyen, J. Chem. Thermodyn. 24, 245 (1992).

16. J. Hopken, S. Faulstich, and M. Moller, Mol. Cryst. Liq. Cryst. Sci. Technol., Sect. A 210, 59 (1992).

17. J. Hopken, M. Moller, and S. Boileau, New Polymeric Mater. 2, 339 (1991).

18. R. D. Chirico, S. E. Knipmeyer, A. Nguyen, N. K. Smith, and W. V. Steele, J. Chem. Thermodyn. 25, 729 (1993).

19. P. Ruelle, E. Sarraf, L. van den Berge, K. Seghers, M. Buchmann, and U. W. Kesselring, Pharm. Acta Helv. 68, 49 (1993).

20. B. F. Rordorf, Proceedings of the 5th International Symposium on Chlorinated Dioxins and Related Compounds, Bayreuth, Germany, September 16-19 (Chemosphere, 1986).

21. P. M. Vilalta, G. S. Hammond, and R. G. Weiss, Langmuir 9, 1910 (1993).

22. J. Hopken and M. Moller, Macromolecules 25, 2482 (1992).

23. P. M. Vilalta and R. G. Weiss, Liq. Cryst. 12, 531 (1992).

24. C. Viney, T. P. Russel, L. E. Depero, and R. J. Twieg, Mol. Cryst.

Liq. Cryst. 168, 63 (1989).

25. I. Furman, R. J. Butcher, R. M. Catchings, and R. G. Weiss, J. Am. Chem. Soc. 114, 6023 (1992).

26. B. M. Sheikh-Ali and R. G. Weiss, Liq. Cryst. 10, 575 (1991).

27. K.-J. Kim, C.-H. Lee, and S.-K. Ryu, J. Chem. Eng. Data 39, 228 (1994).

28. N. Santilli, P. de Filippio, and A. Chianese, J. Chem. Eng. Data 39, 179 (1994).

29. R. Sabbah and O. Pemenzi, C. R. Acad. Sci. Paris, Ser. II 317, 575 (1993).

30. W. V. Steele, R. D. Chirico, A. Nguyen, and S. E. Knipmeyer, J.

Chem. Thermodyn. 26, 515 (1994).

31. R. D. Chirico, B. E. Gammon, I. A. Hossenlopp, and W. V. Steele, J. Chem. Thermodyn. 26, 469 (1994).

32. B. V. Lebedev and A. A. Yevstropov, J. Chem. Thermodyn. 15, 115 (1983).

33. M. D. Contreras Claramonte, A. Parera Vialard, and F. Girela Vilchez, Int. J. Pharm. 94, 23 (1993).

34. Z. Z. Ying and Y. M. Lin, J. Chem. Thermodyn. 22, 617 (1990).

35. V. V. Diky, G. J. Kabo, A. A. Kozyro, A. P. Krasulin, and V. M.

Sevruk, J. Chem. Thermodyn. 25, 1169 (1993).

36. X. Yu, G. L. Zipp, and G. W. R. Davidson III, Pharm. Res. 11, 522 (1994).

37. H. Fujimori and M. Oguni, J. Phys. Chem. Solids 54, 607 (1993).
38. S. S. Chang, J. Chem. Phys. 79, 6229 (1983).

39. G. R. Kelm and A. A. Sakr, Drug Dev. Ind. Pharm. 19, 809 (1993).

40. D. Varech, M. J. Brienne, and J. Jacques, Tetrahedron Lett. 26, 61 (1985).

41. H. Van Bekkum, M. A. Hoefnagel, L. de Lavieter, A. Van Veen, P. E. Verkade, A. Wemmers, B. M. Wepster, J. H. Palm, L. Schafer, H. Dekker, C. Mosselman, and G. Somsen, Rec. Trav. Chim. Pays-Bas 87, 1363 (1968)

42. K. Bystrom, J. Chem. Soc., Faraday Trans. 1 76, 1986 (1980). 43. G. N. D'yakova, I. A. Vasil'ev, and G. V. Petrova, Neftepererab. Neftekhim Moscow 51, (1980). (Chemical Abstracts 92: 170268k.) 44. A. Cuccuru, Thermochim. Acta 11, 247 (1975).

45. C. C. Mjojo, J. Chem. Soc., Faraday Trans. 2 75, 692 (1979). 46. P. Corradini, A. Frasci, and E. Martuscelli, J. Chem. Soc. D (14) 778 (1969).

47. G. Borgen, Acta Chem. Scand. 29B, 265 (1975).

48. B. V. Lebedev, V. I. Milov, L. Y. Tsvetkova, N. K. Lebedev, E. G. Kiparisova, and A. A. Evstropov, Zh. Fiz. Khim. 49, 1592 (1975).

49. S. J. Ashcroft, J. Chem. Eng. Data 21, 397 (1976).

50. J. F. Messerly, H. L. Finke, A. G. Osborn, and D. R. Douslin, J. Chem. Thermodyn. 7, 1029 (1975).

51. A. M. I. Ahmed and R. G. Eades, J. Chem. Soc., Faraday Trans. 2 68, 2017 (1972)

52. I. A. Vasil'ev and A. D. Korkhov, Zh. Fiz. Khim. 47, 2710 (1973).

53. Y. K. Shaulov and E. S. Yakubov, Zh. Fiz. Khim. 45, 188 (1971).

54. A. J. Valerga and J. E. Kilpatrick, J. Chem. Phys. 52, 4545 (1970).

55. P. N. Nikolaev and I. B. Rabinovich, Zh. Fiz. Khim. 49, 2191 (1967).

56. J. H. Magill, J. Chem. Phys. 47, 2802 (1967).

57. P. Goursot and E. F. Westrum, Jr., C. R. Acad. Sci., Paris, Ser. C 266, 667 (1968).

58. M. A. Soulie, P. Goursot, A. Peneloux, and J. Metzger, J. Chim. Phys. Physicochim. Biol. 66, 607 (1969).

59. P. Goursot and E. F. Westrum, Jr., C. R. Acad. Sci., Paris, Ser. C 266, 590 (1968).

60. T. P. Melia and R. Merrifield, J. Appl. Chem. (London) 19, 79 (1969). 61. R. Meyer and J. Metzger, C. R. Acad. Sci., Paris, Ser. C 263, 1333 (1966).

62. K. I. Geidarov, O. I. Dzhafarov, K. A. Karasharli, and V. N. Kostryukov, Zh. Fiz. Khim. 48, 1058 (1974).

63. K. I. Geidarov, O. I. Dzhafarov, K. A. Karasharli, and V. N. Kostryukov, Zh. Fiz. Khim. 47, 275 (1973).

64. A. Gonthier-Vassal and H. Szwarc, Thermochim. Acta 202, 87 (1992). 65. R. J. L. Andon and J. F. Martin, J. Chem. Soc., Faraday Trans. 1 69, 761 (1973).

66. R. J. L. Andon, J. F. Counsell, D. A. Lee, and J. F. Martin, J. Chem. Soc., Faraday Trans. 1 70, 1914 (1974).

67. H. W. Starkweather, Jr., Marcomolecules 19, 1131 (1986).

68. T. P. Russell, J. F. Rabolt, R. J. Twieg, R. L. Siemens, and B. L. Farmer, Macromolecules 19, 1135 (1986).

69. R. D. Chirico, I. A. Hossenlopp, B. E. Gammon, S. E. Knipmeyer, and W. V. Steele, J. Chem. Thermodyn. 26, 1187 (1994).

70. R. D. Chirico, I. A. Hossenlopp, B. E. Gammon, S. E. Knipmeyer, and W. V. Steele, J. Chem. Thermodyn. 26, 1219 (1994).

71. N. V. Karyakin, K. G. Shvetsova, and E. S. Dzharimova, Termodin. Org. Soedin. 33, CA 99:129172f (1982).

72. R. J. L. Andon, J. F. Counsell, J. L. Hales, E. B. Lees, and J. F. Martin, J. Chem. Soc. Sect. A, (10) 2357 (1968).

73. J. F. Counsell, E. B. Lees, and J. F. Martin, J. Chem. Soc., Sect. A, (10) 1819 (1968).

74. R. J. L. Andon, J. F. Counsell, D. A. Lee, and J. F. Martin, J. Chem. Soc., Faraday Trans. 1, 69, 1721 (1973).

75. J. F. Counsell, D. A. Lee, and J. F. Martin, J. Chem. Soc., Sect. A, (2) 313 (1971)

76. R. J. L. Andon, J. E. Connett, J. F. Counsell, E. B. Lees, and J. F. Martin, J. Chem. Soc., Sect. A, (4) 661 (1971). 
Table 8. References to Tables 5, 6, and 7-Continued

77. J. F. Counsell, J. L. Hales, E. B. Lees, and J. F. Martin, J. Chem. Soc., Sect. A, 2994 (1968).

78. A. A. Trapeznikov and T. A. Lomonosova, Zh. Fiz. Khim. 44, 2121 (1970).

79. B. Kalinowska and A. W. Jackowski, J. Chem. Thermodyn. 18, 715 (1986).

80. C. G. Dekruif, J. C. Van Miltenburg, and J. G. Blok, J. Chem. Thermodyn. 15, 129 (1983).

81. K. Adachi, H. Suga, and S. Seki, Bull. Chem. Soc. Jpn. 41, 1073 (1968).

82. L. El Watik and R. Sabbah, Bull. Soc. Chim. France 128, 344 (1991). 83. M. S. Khanna, S. C. Khetarpal, K. Lal, and H. L. Bhatnagar, Ind. J. Chem. 20A, 544 (1981).

84. V. G. Vasil'ev and B. V. Lebedev, Zh. Obshch. Khim. 59, 2415 (1989).

85. J. J. Pinvidic, A. Gonthier-Vassal, H. Szwarc, R. Ceolin, P. Toffoli, J. M. Teulon, and C. Guechot, Thermochim. Acta 153, 37 (1989).

86. G. Kossmehl and D. Budwell, Z. Naturforsch 42B, 478 (1978).

87. P. Eiselt, S. Denzinger, and H.-W. Schmidt, Liq. Cryst. 18, 257 (1995).

88. Y. K. Kuchhal, R. N. Shukla, and A. B. Biswas, Thermochim. Acta 31, 61 (1979).

89. I. A. Vasil'ev and A. D. Korkhov, Tr. Khim. Khim. Tekhnol. 103, CA 82:65233r (1974).

90. H. P. Frank, K. Krzemicki, and H. Voellenkle, Chem.-Ztg. 97, 206 (1973).

91. S. S. Chang and A. B. Bestul, J. Chem. Phys. 56, 503 (1972).

92. K. Kishimoto, H. Suga, and S. Seki, Bull. Chem. Soc. Jpn. 46, 3020 (1973).

93. G. N. D'yakova and I. A. Vasil'ev, Neftepererab. Neftekhim. (Moscow), 51, CA 92:170269m (1980).

94. G. T. Furukawa, M. L. Reilly, H. H. Piccirelli, and M. Tenenbaum, J. Res. Natl. Bur. Std. A68, 367 (1964).

95. R. C. Chirico, S. E. Knipmeyer, A. Nguyen, and W. V. Steele, J. Chem. Thermodyn. 28, 431 (1991).

96. A. M. Gibin, N. D. Grishnova, A. V. Gusev, A. N. Moiseev, I. I. Remeshkova, and Y. M. Salganskii, Vysokochist. Veshchestva 28, CA 109: 136102 (1987)

97. J. J. Mallon and S. W. Kanto, Macromolecules, 22, 2070 (1989). 98. S. A. Mekhtiev, K. A. Karasharli, and D. I. Dzhafarov, Russ. J. Phys. Chem. 49, 259 (1975).

99. S. A. Mekhtiev, K. A. Karasharli, and D. I. Dzhafarov, Russ. J. Phys. Chem. 49, 914 (1975)

100. B. V. Lebedev, L. Y. Tsvetkova, E. G. Kiparisova, and N. K. Lebedev, Russ. J. Phys. Chem. 49, 1265 (1975).

101. P. P. Inozemtsev, A. G. Liakumovich, and Z. D. Gracheva, Russ. J. Phys. Chem. 46, 914 (1972)

102. V. P. Privalko, Russ. J. Phys. Chem. 45, 900 (1971).

103. V. N. Kostryukov, O. P. Samorukov, N. K. Samorukova, and E. K. Chaplygina, Russ. J. Phys. Chem. 49, 944 (1975).

104. B. V. Lebedev, A. A. Evstropov, G. B. Sadikov, and L. F. Larina, Russ. J. Phys. Chem. 51, 762 (1977).

105. J. F. Messerly, H. L. Finke, and S. S. Todd, J. Chem. Thermodyn. 6 635 (1974).

106. H. G. Carlson and E. F. Westrum, Jr., J. Chem. Eng. Data 10, 134 (1965).

107. G. Borgen and J. Dale, Acta Chem. Scand. 26, 1149 (1972).

108. E. Augdahl, G. Borgen, J. Dale, and J. Krane, Acta Chem. Scand. 28B, 125 (1974).

109. G. Borgen, Acta Chem. Scand. 28B, 13 (1974)

110. S. L. Björnstad, G. Borgen, and G. Gaupset, Acta Chem. Scand. 28B, 821 (1974)

111. G. Borgen and J. Dale, Acta Chem. Scand. 30B, 711 (1976).

112. S. L. Björnstad, G. Borgen, J. Dale, and G. Gaupset, Acta Chem. Scand. 29B, 320 (1975).

113. S. L. Björnstad and G. Borgen, Acta Chem. Scand. 29B, 13 (1975).
114. T. Alvik, G. Borgen, and J. Dale, Acta Chem. Scand. 26, 1805 (1972)

115. G. Borgen and J. Dale, Acta Chem. Scand. 26, 1799 (1972).

116. G. Borgen and J. Dale, J. Chem. Soc., Chem. Commun. D (9) 447

(1969)

117. J. Dale and T. Ekeland, Acta Chem. Scand. 27, 1519 (1973).

118. S. C. Khetarpal, K. Lal, and H. L. Bhatnagar, Ind. J. Chem. 20A, 446 (1981).

119. G. Borgen and J. Dale, J. Chem. Soc., Chem. Commun. D (20) 1340 (1970).

120. J. Dale and P. O. Kristiansen, Acta Chem. Scand. 26, 1471 (1972).

121. T. Alvik and J. Dale, Acta Chem. Scand. 25, 2131 (1971).

122. J. M. Rosen, J. R. Holden, and H. T. Simmons, Sr., Microscope 19, 151 (1971).

123. U. Domanska, J. Serwatowski, A. Sporzynski, and M. Dabrowski, Thermochim. Acta 222, 279 (1993).

124. G. J. Kabo, A. A. Kozyro, V. V. Diky, and V. V. Simirsky, J. Chem. Eng. Data 40, 371 (1995).

125. H. Utschick, G. Bachmann, and H. Kapitza, Chem. Technik. 26, 423 (1974)

126. A. P. Melissaris and M. H. Litt, Macromolecules 27, 888 (1994).

127. G. J. Kabo, A. A. Kozyro, A. P. Marchand, V. V. Diky, V. V.

Simirsky, L. S. Ivashkevich, A. P. Krasulin, V. M. Sevruk, and M. L.

Frenkel, J. Chem. Thermodyn. 26, 129 (1994).

128. B. Lebedev, V. Vasil'yev, and N. Novosyolova, Makromol. Chem.

194, 739 (1993)

129. B. Lebedev, N. Smirnova, Y. Kiparisova, and K. Makovetsky, Makromol. Chem. 193, 1399 (1992).

130. H. Utschick, G. Bachmann, and H. Kapitza, Chem. Technik. 26, 422 (1974)

131. J.-I. Jin, C.-S. Kang, and B. Y. Chung, Bull. Korean Chem. Soc. 11, 245 (1990).

132. B. V. Lebedev, T. A. Bykova, N. N. Smirnova, and T. G. Kulagina, Zhr. Obshch. Khim. 52, 2630 (1982).

133. N. K. Lebedev, E. G. Kiparisova, B. V. Lebedev, A. M. Sladkov, and N. A. Vasmeva, Bull. Acad. Sci. USSR, Div. Chem. Sci. 29, 374 (1980) (English translation).

134. B. V. Lebedev, T. A. Bykova, E. G. Kiparisova, Y. A.

Chernomordik, A. S. Kurapov, and V. A. Sergeev, Bull. Acad. Sci. USSR, Div. Chem. Sci. 34, 274 (1985) (English translation).

135. B. V. Lebedev, T. A. Bykova, E. G. Kiparisova, Ts. M. Frenkel, A. M. Fainleib, and V. A. Pankratov, Bull. Acad. Sci. USSR, Div. Chem. Sci. 37, 1082 (1988) (English translation).

136. J. A. Dean, Lange's Handbook of Chemistry, 13th ed. (McGrawHill, New York, 1985).

137. G. D. Maury and J. Masse, J. Therm. Anal. 31, 1263 (1986).

138. G. Y. Kabo, E. A. Miroshnichenko, M. L. Frenkel, A. A. Koxyro, V. V. Simirskii, A. P. Krasulin, V. P. Vorob'eva, and Y. A. Lebedev, Bull. Acad. Sci. USSR, Div. Chem. Sci. 39, 662 (1990) (English translation). 139. U. S. Rai and S. George, Cryst. Res. Technol. 26, 511 (1991). 140. S. M. Kelly, M. Schadt, and H. Seiberle, Liq. Cryst. 18, 581 (1995) 141. S. Miyajima, A. Nakazato, N. Sakoda, and T. Chiba, Liq. Cryst. 18, 651 (1995).

142. J. Kalinowski, Z. Dreger, J. Godlewski, and P. Mondalski, Chem.

Phys. Lett. 172, 522 (1990).

143. A. K. Labban, E. F. Westrum, Jr., and J. A. R. Cheda, Can. J. Chem. 69, 1796 (1991).

144. T. Clark, M. A. McKervey, H. Mackle, and J. Rooney, J. Chem. Soc., Faraday Trans. 1 70, 1279 (1977).

145. N. T. Kawai, D. F. R. Gilson, and I. S. Butler, Can. J. Chem. 69, 1758 (1991)

146. T. Clark, T. Mc. O. Knox, H. Mackle, and M. A. McKervey, J. Chem. Soc., Faraday Trans 1 73, 1224 (1977).

147. M. Pickering, J. Rylance, R. W. H. Small, and D. Stubley, Acta Crystallogr., Sect. B 47, 782 (1991).

148. M. Foulon, J. P. Amoureux, J. L. Sauvajol, J. P. Cavrot, and M. Muller, J. Phys. C 17, 4213 (1984). 
Table 8. References to Tables 5, 6, and 7-Continued

149. P. D. Harvey, D. F. R. Gilson, and I. S. Butler, J. Phys. Chem. 90, 136 (1986).

150. M. A. White, R. E. Wasylishen, P. E. Eaton, Y. Xiong, K. Pramod, and N. Nodari, J. Phys. Chem. 96, 421 (1992).

151. P. Navard and J. M. Haudin, J. Thermal Anal. 22, 107 (1981).

152. M. Balty, S. Mraw, L. A. K. Staveley, A. H. Overs, M. C. Owen, R. K. Thomas, and J. W. White, Mol. Phys. 45, 1015 (1982).

153. Z. A. Guseinov, K. A. Karasharli, O. I. Dzhafarov, G. G. Nurullaev, N. S. Nametkin, L. E. Gusel'nikov, E. A. Volnina, E. N. Burdasov, and V. M. Vdovin, Dokl. Acad. Nauk SSSR 222, 1369 (1975).

154. J. A. R. Cheda and E. F. Westrum, Jr., J. Phys. Chem. 98, 2482 (1994).

155. K. Saito, T. Atake, and H. Chihara, Bull. Chem. Soc. Jpn. 61, 2327 (1988).

156. N. Nakamura, H. Suga, and S. Seki, Bull. Chem. Soc. Jpn. 53, 2755 (1980).

157. K. Saito, T. Atake, and H. Chihara, J. Chem. Thermodyn. 17, 539 (1985).

158. K. Kishimoto, H. Suga, and S. Seki, Bull. Chem. Soc. Jpn. 51, 1691 (1978).

159. M. A. White and A. Perrott, J. Solid State Chem. 90, 87 (1991).

160. R. Sabbah and L. El Watik, Can. J. Chem. 70, 24 (1992).

161. B. Lebedev and N. Smirnova, Macromol. Chem. Phys. 195, 35 (1994).

162. B. Lebedev, N. Smirnova, N. Novosyolova, K. Makovetskii, and I. Ostrovskaya, Macromol. Chem. Phys. 195, 1807 (1994).

163. M. A. White and A. Perrott, Can. J. Chem. 66, 729 (1988).

164. A. C. Shah and N. J. Britten, J. Pharm. Pharmacol. 39, 736 (1986).

165. J. F. Messerly, S. S. Todd, H. L. Finke, S. H. Lee-Bechtold, G. B.

Guthrie, W. V. Steele, and R. D. Chirico, J. Chem. Thermodyn. 22, 1107 (1990).

166. M. S. Sheiman, I. B. Rabinovich, V. P. Nistratov, G. P. Kamelova, E. N. Karataev, and I. A. Feshchenko, Zh. Fiz. Khim. 63, 836 (1989). 167. V. P. Nistratov, I. B. Rabinovich, M. S. Sheiman, N. N. Smirnova, I. A. Zelyaev, and I. A. Feshchenko, Zh. Fiz. Khim. 63, 1779 (1989). 168. V. G. Yasil'ev, T. A. Bykova, and B. V. Lebedev, Zh. Fiz. Khim. 65, 51 (1991).

169. A. A. Kozyro, V. V. Simirskii, G. Y. Kabo, M. L. Frenkel, A. P. Krasulin, V. M. Sevruk, and N. A. Sokolov, Zh. Fiz. Khim. 64, 2360 (1990).

170. I. B. Rabinovich, M. S. Sheiman, G. P. Kamelova, V. P. Nistratov, and E. N. Karataev, Zh. Fiz. Khim. 65, 2071 (1991).

171. V. P. Nistratov, M. S. Sheiman, I. B. Rabinovich, V. G. Vasil'ev, E. N. Karataev, and I. A. Feshchenko, Zh. Fiz. Khim. 62, 2219 (1988). 172. S. R. Salman and K. F. Abas, Thermochim. Acta 142, 245 (1989).

173. V. N. Lushnikov, Y. I. Rubstov, L. T. Dremenko, and A. M. Korolev, Zh. Fiz. Khim. 62, 1209 (1988).

174. A. A. Kozyro, V. V. Simirskii, A. P. Krasulin, V. M. Sevruk, G. Y. Kabo, M. L. Frenkel, P. N. Gaponik, and Y. V. Grigor'ev, Zh. Fiz. Khim. 64, 2360 (1990).

175. M. Cao, J. Wesson, K. Loufakis, B. Wunderlich, and M. Moller, Mol. Cryst. Liq. Cryst. 140, 231 (1986).

176. A. Cuccuru, Thermochim. Acta 13, 96 (1975).

177. V. P. Privalko, G. A. Puchkovskaya, E. N. Shermatov, and A. A. Yakubov, Mol. Cryst. Liq. Cryst. 126, 289 (1985).

178. A. J. Herbert, Trans. Faraday Soc. 63, 555 (1967).

179. F. Casellato, C. Vecchi, and A. Girelli, Thermochim. Acta 21, 195 (1977).

180. N. B. Singh and M. E. Glicksman, Thermochim. Acta 159, 93 (1990).

181. H. Drotloff and M. Moller, Thermochim. Acta 112, 57 (1987).

182. A. El Moussaoui, A. Chauvet, and J. Masse, J. Thermal Anal. 39, 619 (1993).

183. G. J. Kabo, A. A. Kozyro, V. V. Diky, V. V. Simirsky, L. S. Ivashkevich, A. P. Krasulin, V. M. Sevruk, A. P. Marchand, and M. Frenkel, J. Chem. Thermodyn. 27, 707 (1995).
184. H. L. Finke, J. P. McCullough, J. F. Messerly, A. Osborn, and D. R. Douslin, J. Chem. Thermodyn. 4, 477 (1972).

185. L. A. Torres, G. Gudiño, I. Hernandez-Contreras, M. E. Ochoa, N. Farfan, and R. L. Santillan, J. Chem. Thermodyn. 27, 779 (1995).

186. M. Dabrowski, U. Domanska, J. Serwatowski, and A. Sporzynski, Thermochim. Acta 250, 19 (1995).

187. S. Larsen and K. Marthi, Acta Cryst., Sect. B B50, 373 (1994).

188. R. Sabbah and M. Gouali, Austr. J. Chem. 47, 1651 (1994).

189. G. Hakvoort, Calorim. Anal. Therm. 24, 177 (1993).

190. K. Kobashi and M. Oguni, J. Chem. Thermodyn. 27, 979 (1995).

191. G. Wojcik, L. Toupet, C. Gors, and M. Foulon, Phys. Status Solids A 147, 99 (1995).

192. J. Pirsch, Monatsh. Chem. 97, 260 (1966).

193. S. A. Skackelford and J. F. Goldman, Propellants, Explos., Pyrotech. 20, 1 (1995).

194. P. Bustamante and B. Escalera, J. Pharm. Pharmacol. 47, 550 (1995).

195. T. M. Juang, Y. N. Chen, S. H. Lung, Y. H. Lu, C. S. Hsu, and S.

T. Wu, Liq. Cryst. 15, 529 (1993).

196. C. S. Hsu, K. T. Tsay, A. C. Chang, S. R. Wang, and S. T. Wu, Liq. Cryst. 19, 409 (1995).

197. J. M. Bell and J. P. Sawyer, J. Ind. Eng. Chem. 11, 1025 (1919). 198. A. Krajewska and K. Pigon, Thermochim. Acta 41, 187 (1980). 199. P. Bret-Dibat and A. Lichanot, Thermochim. Acta 147, 261 (1989). 200. B. V. Lebedev, T. G. Kulagina, V. I. Telnoy, and V. G. Vasil'ev, Macromol. Chem. Phys. 196, 3487 (1995).

201. M.-J. Lee, P.-J. Lien, and W.-K. Huang, Ind. Eng. Chem. Res. 33, 2853 (1994).

202. U. Domanska, W. C. Moollan, and T. M. Letcher, J. Chem. Eng.

Data 41, 261 (1996).

203. A. Kristl and G. Vesnaver, J. Chem. Soc., Faraday Trans. 91, 995 (1995).

204. T. M. R. Maria, F. S. Costa, M. L. P. Leitao, and J. S. Redinha, Thermochim. Acta 269/270, 405 (1995).

205. W. V. Steele, R. D. Chirico, S. E. Knipmeyer, and A. Nguyen, J. Chem. Thermodyn. 23, 957 (1991).

206. B. V. Lebedev, T. A. Bykova, E. G. Kiparisova, and V. G. Vasil'yev, Macromol. Chem. Phys. 197, 1553 (1996).

207. W. V. Steele, R. D. Chirico, A. B. Cowell, A. Nguyen, and S. E. Knipmeyer, J. Chem. Thermodyn. 27, 1407 (1995).

208. T. G. Kulagina and B. V. Lebedev, Journees Mediterranennes Calorimetrie d'Analyze Thermique 24, 2535 (1993).

209. F. Espitalier, B. Biscans, P. S. Peyrigain, and C. Laguerie, Fluid Phase Equilibr. 113, 151 (1995).

210. A. Hammami and A. K. Mehrotra, Fluid Phase Equilibr. 111, 253 (1995).

211. H. E. Gallis, F. Bougrioua, H. A. J. Oonk, P. J. van Ekeren, and J. C. van Miltenburg, Thermochim. Acta 274, 231 (1996).

212. B. Belhachemi, W. Ufnalsky, and Z. Derriche, J. Chim. Phys. 93, 1117 (1996)

213. H. E. Gallis, G. J. K. van den Berg, and H. A. J. Oonk, J. Chem. Eng. Data 41, 1303 (1996).

214. C. Fiege, R. Joh, M. Petri, and J. Gmehling, J. Chem. Eng. Data 41 1431 (1996).

215. W. E. Acree, Jr., Thermochim. Acta 189, 37 (1991) and references therein.

216. E. S. Domalski and E. D. Hearing, J. Phys. Chem. Ref. Data 25, 1 (1996) and references therein.

217. W. E. Acree, Jr., Thermochim. Acta 219, 97 (1993) and references therein.

218. R. H. Manzo and A. A. Ahumanda, J. Pharm. Sci. 79, 1109 (1990). 219. A. Regocz, A. Chmielewska, T. Pelpinska, and P. Kowalski, Pharmazie 49, 371 (1994).

220. J. S. Chickos, C. M. Braton, D. G. Hesse, and J. F. Liebman, J. Org. Chem. 56, 927 (1991) and references and supplemental material therein. 
Table 8. References to Tables 5, 6, and 7-Continued

221. J. R. Donnelly, L. A. Drewes, R. L. Johnson, W. D. Munslow, K. K. Knapp, and G. W. Sovocool, Thermochim. Acta 167, 155 (1990).

222. A. Ksiazczak and I. Nagata, Thermochim. Acta 254, 31 (1995).

223. R. Sabbah and M. Gouali, Can. J. Chem. 74, 500 (1996).

224. C. Mosselman and J. Mouric, J. Chem. Thermodyn. 6, 477 (1974).

225. Y. Oyumi and T. B. Brill, Thermochim. Acta 116, 125 (1987).

226. J. S. Chickos, D. L. Garin, M. Hitt, and G. Schilling, Tetrahedron

37, 2255 (1981).

227. E. S. Domalski and E. D. Hearing, J. Phys. Chem. Ref. Data 19, 881 (1990).

228. T. N. Masalitinova, T. P. Oleinikova, V. L. Ryadnenko, N. N. Kiseleva, and N. D. Lebedeva, Zh. Prikl. Khim. (Leningrad) 54, 1799 (1981).

229. V. V. Kiky, G. J. Kabo, A. A. Kozyro, A. P. Krasulin, and V. M. Sevruk, J. Chem. Thermodyn. 26, 1001 (1994).

230. K. B. Wiberg, D. J. Wasserman, E. J. Martin, and M. A. Murcko, J. Am. Chem. Soc. 107, 6019 (1985).

231. J. G. Priest, E. M. Wooley, J. B. Ott, and J. R. Goates, J. Chem. Thermodyn. 15, 357 (1983).

232. C. Plato and A. R. Glasgow, Jr., Anal. Chem. 41, 330 (1969).

233. N. A. Armstrong, K. C. James, and C. K. Wong, J. Pharm.

Pharmacol. 31, 627 (1979).

234. J. C. Van Miltenburg, A. Alvarez-Larena, M. Labrador, L. Palacios, J. Rodriquez-Romero, E. Tauler, and E. Estop, Thermochim. Acta 273, 31 (1996).

235. G. Poeti, E. Faneli, and M. J. Braghetti, Therm. Anal. 24, 273 (1982).

236. T. Gondova, P. Kralik, and J. Gonda, Thermochim. Acta 156, 147 (1989).

237. G. Borgen, Acta Chem. Scand. B28, 13 (1974).

238. H. Mackle, D. V. McNally, and W. V. Steele, Trans. Faraday Soc. 65, 2060 (1969).

239. R. H. Manzo and A. A. Ahumada, J. Pharm. Sci. 79, 1109 (1990).

240. W. Zielenkiewicz, A. Zielenkiewicz, and K. L. Wierchowski, J.

Solution Chem. 23, 1125 (1994).

241. A. Chuvet, G. De Maury, A. Terol, and J. Masse, Thermochim. Acta 97, 143 (1986).

242. B. K. Braxton and J. H. Rytting, Thermochim. Acta 154, 27 (1989). 243. R. van der Geest, R. M. Kellogg, and H. Wynberg, Recueil Travaux Chimiques Pays-Bas 109, 197 (1990).

244. J. S. Chickos, S. Hosseini, D. Hesse, G. Nichols, and P. Webb, Thermochim. Acta 313, 101 (1998).

245. H.-D. Beckhaus, B. Dogan, J. Pakusch, S. Verekin, and C. Ruchardt, Chem. Ber. 123, 2153 (1990).

246. D. R. Kirklin and E. S. Domalski, J. Chem. Thermodyn. 20, 743 (1988).

247. Y. Dozen, S. Fujishima, and H. Shingu, Thermochim. Acta 25, 209 (1978).

248. B. Schneider, J. Kvarda, P. Schmidt, M. Suchoparek, and I. Prokopova, Collect. Czech. Chem. Commun. 58, 2403 (1993).

249. S. Miyagishi, S. Matumura, T. Asakawa, and M. Nishida, Bull Chem. Jpn. 59, 557 (1986).

250. D. W. Scott, J. P. McCullough, W. D. Good, J. F. Merrerly, R. E. Pennington, T. C. Kincheloe, I. A. Hossenlopp, D. R. Douslin, and G. Waddington, J. Am. Chem. Soc. 78, 5457 (1956).

251. R. Brettle, D. A. Dunmur, C. M. Marson, M. Pinol, and K. Toriyama, Liq. Cryst. 13, 515 (1993).

252. K. Rakus, S. P. Verevkin, J. Schatzer, H.-D. Beckhaus, and C. Ruechardt, Chem. Ber. 127, 1095 (1994).

253. R. Welle, S. P. Verevkin, M. Keller, H.-D. Beckhaus, and C. Ruechardt, Chem. Ber. 127, 697 (1994).

254. H.-D. Beckhaus, C. Ruechardt, D. R. Lagerwall, L. A. Pauette, F. Wahl, and H. Prinzbach, J. Am. Chem. Soc. 116, 11775 (1994).

255. F. Casellato, C. Vecchi, and A. Girell, Thermochim. Acta 6, 361 (1973).
256. H. Kambe, I. Mita, and R. Yokata, Thermal Analysis, Volume 3, Proceedings Third ICTAC (Davos Swizz, 1971), pp. 387-395.

257. C. W. Jefford, A. Jaber, J. Boukouvalas, and P. Tissot, Thermochim. Acta 188, 337 (1991).

258. P. S. Engel, C. Wang, Y. Chen, C. Ruechardt, and H.-D. Beckhaus, J. Am. Chem. Soc. 115, 65 (1993).

259. M. Nolke, S. P. Verevkin, H.-D. Beckhaus, and C. Ruechardt, Liebigs Ann. (1) 41 (1995).

260. C. Carfagna, V. Busico, V. Salerno, and M. Vacatello, Thermochim. Acta 37, 31 (1980).

261. Z. Z. Liu, X. D. Guo, L. E. Straub, G. Erdos, R. J. Prankerd, R. J. Gonzalez-Rothi, and H. Schreier, Drug Design Discovery 8, 57 (1991). 262. P. Ruelle, E. Sarraf, L. van den Berge, K. Seghers, M. Buchmann, and U. W. Kesselring, Pharm. Acta. Helv. 68, 49 (1993).

263. P. M. Vilalta, G. S. Hammond, and R. G. Weiss, Langmuir 9, 1910 (1993).

264. G. W. Smith, Mol. Cryst. Liq. Cryst. 64, 15 (1979).

265. J. D. Hoffman and B. F. Decker, J. Chem. Phys. 57, 520 (1953).

266. G. D. Mendenhall, S. X. Liang, and E. H.-T. Chen, J. Org. Chem.

55, 3697 (1990).

267. G. Latterman and H. Hoecker, Mol. Cryst. Liq. Cryst. 133, 245 (1986).

268. D. Lourdin, A. H. Roux, J.-P. E. Grolier, and J.-M. Buisine,

Thermochim. Acta 204, 99 (1992).

269. J. Hulnick, K. Van Miltenburg, H. A. Oonk, and A. J. Schuljff, J.

Chem. Eng. Data 34, 99 (1989).

270. W. Kreis and R. H. Wood, J. Chem. Thermodyn. 1, 523 (1969).

271. H. G. M. De Wit, C. G. De Kruif, and J. C. Van Miltenburg, J.

Chem. Thermodyn. 15, 891 (1983).

272. D. Borigeard, N. Le Calve, A. Novak, and B. C. Nguyen, Mol.

Cryst. Liq. Cryst. 44, 113 (1978).

273. M. Leclercq, A. Collet, and J. Jacques, Tetrahedron 32, 821 (1976).

274. Z. Zhang and M. L. Yang, Thermochim. Acta 169, 263 (1990).

275. R. E. Pennington, H. L. Finke, W. N. Hubbard, J. F. Messerly, F. R.

Frow, I. A. Hossenlopp, and G. Waddington, J. Am. Chem. Soc. 78, 2055 (1956).

276. W. K. Busfield, K. J. Ivin, H. Mackel, and P. A. G. O'Hare, Trans. Faraday Soc. 56, 1058 (1960).

277. G. S. Parks, H. M. Huffman, and M. Barmore, J. Am. Chem. Soc. 55, 2733 (1933).

278. D. Gerro, G. Della Gatta, and G. Barone, J. Therm. Anal. 34, 835 (1988).

279. N. D. Lebedeva and Y. A. Katin, J. Appl. Chem. (USSR) 46, 2131 (1973).

280. N. D. Lebedeva, V. L. Ryadnenko, and I. N. Kuznetsova, Russ. J.

Phys. Chem. 45, 549 (1971).

281. D. W. Scott, J. P. McCullough, W. N. Hubbard, J. F. Messerly, I. A.

Hossenlopp, F. R. Frow, and G. Waddington, J. Am. Chem. Soc. 78, 5463 (1956).

282. E. S. Domalski, W. H. Evans, and E. D. Hearing, J. Phys. Chem.

Ref. Data 13, suppl. 1 (1984).

283. D. R. O'Rourke and S. C. Mraw, J. Chem. Thermodyn. 15, 489 (1983).

284. D. M. McEachern, O. Sandoval, and J. C. Iniguez, J. Chem.

Thermodyn. 7, 1029 (1995).

285. F.-W. Schlze, H.-J. Petrick, H. K. Canmenga, and H. Z. Klinge, Z. Phys. Chem. (Wiesboden) 107, 1 (1977).

286. R. D. Chirico, B. E. Gammon, S. E. Kripmeyer, A. Nguyen, M. M. Strube, C. Tsonopoulos, and W. V. Steele, J. Chem. Thermodyn. 22, 1075 (1990).

287. A. S. Carson, D. H. Fine, P. Gray, and P. G. Laye, J. Chem. Soc. Sect. B(8) 1611 (1971).

288. W. T. Berg, D. W. Hubbard, S. S. Todd, J. F. Messerly, I. A. Hassenlopp, A. Osborn, D. R. Douslin, and J. P. McCullough, J. Phys. Chem. 65, 1425 (1961).

289. G. Kratt, H.-D. Beckhaus, W. Behrnohr, and C. Ruechardt, Thermochim. Acta 62, 279 (1983). 
Table 8. References to Tables 5, 6, and 7-Continued

290. G. S. Parks, G. E. Moore, M. L. Renquist, B. F. Naylor, L. A. McClaine, P. S. Fujii, and J. A. Hatton, J. Am. Chem. Soc. 71, 3386 (1949). 291. C. C. Cypcar, G. Johnson, and L. J. Mathias, Polym. Prepr. (Am. Chem. Soc., Div. Polym. Chem.) 36, 402 (1995).

292. M. Sakiyama and A. Imamura, Thermochim. Acta 142, 365 (1989). 293. H. E. Gallis, G. J. K. van den Berg, and H. A. J. Oonk, J. Chem. Eng. Data 41, 1303 (1996).

294. A. E. Sokolovskii and A. K. Baev, Russ. J. Phys. Chem. 58, 1635 (1984).

295. T. Oishi, H. Horie, and H. Shuyama, Polmy. J. 25, 781 (1993).

296. S. D. Roy and G. L. Flynn, Pharm. Res. 5, 580 (1988).

297. B. Lebedev, N. Smirnova, and V. Telnoy, Macromol. Chem. Phys.

197, 3807 (1996).

298. M. V. Roux, P. Jimenez, J. Z. Davalos, J.-L. M. Abboud, and M. T. Molina, J. Chem. Thermodyn. 28, 1029 (1998).

299. M. S. Sheiman, G. P. Kamelova, K. G. Shvetsova, and V. P.

Nistratov, Russ. J. Phys. Chem. 70, 1105 (1996).

300. V. I. Tel'noi, M. S. Sheiman, V. N. Larina, and G. P. Kamelova, Russ. J. Phys. Chem. 70, 1261 (1996).

301. M.-J. Lee, Y.-K. Chang, H.-M. Lin, and C.-H. Chen, J. Chem. Eng. Data 42, 349 (1997).

302. P. Kerkoc, S. Lochran, R. T. Bailey, F. R. Cruickshank, D. Pugh, and J. Sherwood, J. Appl. Phys. 81, 624 (1997).

303. J. Zhang, R. Hu, C. Zhu, F. Geng, and Q. Long, Theory Pract. Energ. Mater. 133, CA 126:133206p (1996).

304. V. F. Ur'yash, O. V. Ronina, E. N. Karataev, I. V. Zimina, T. V. Kuznetsova, T. V. Petrun, I. A. Feshchenko, and A. D. Zorin,

Metalloogranicheskaya Khim. 4, 1050 (1991).

305. I. Ciocazanu and V. Meltzer, J. Thermal Anal. 47, 1755 (1996).

306. T. O. Luddecke and J. W. Magee, Int. J. Thermophys. 17, 823

(1996).

307. J. Yuan, G.-L. Zhang, D.-Y. Huang, and H.-Z. Zhang, Liq. Cryst. 6, 693 (1997)

308. N. N. Smirnova, B. V. Lebedev, and V. G. Vasil'ev, Zh. Obshch. Khim. 66, 199 (1997).

309. B. V. Lebedev and T. G. Kulagina, J. Chem. Thermodyn. 29, 595 (1997).

310. R. M. Varushchenko, A. I. Druzhinina, and E. L. Sorkin, J. Chem. Thermodyn. 29, 623 (1997).

311. T. Ohta, O. Yamamuro, and T. Matsuo, J. Phys. Chem. 99, 2403 (1995).

312. S. Takahara, O. Yamamuro, and T. Matsuo, J. Phys. Chem. 99, 9589 (1995).

313. Y. V. Maksimuk, G. J. Kabo, A. A. Kozyro, V. V. Simirsky, A. P. Krasulin, and V. M. Sevruk, J. Chem. Thermodyn. 29, 687 (1997).

314. L. I. Marachuk, A. A. Kozyro, and V. V. Simirsky, Zh. Prykl. Khim. 64, 875 (1992).

315. G. L. Baughman and E. J. Weber, Dyes Pigments 16, 261 (1991).

316. C.-F. Shieh, D. McNally, and R. H. Boyd, Tetrahedron 25, 3653 (1969).

317. G. Van Dyke Tiers, J. Org. Chem. 26, 2539 (1961).

318. R. E. Pincock, K. R. Wilson, and T. E. Kiovsky, J. Am. Chem. Soc. 89, 6890 (1967).

319. A. Burger, K. T. Koller, and W. M. Schiermeir, Eur. J. Pharm. Biopharm. 42, 142 (1996).

320. A. L. Stinchcomb, R. Dua, A. Paliwal, R. W. Woodard, and G. L. Flynn, Pharm. Res. 12, 1526 (1995).

321. U. Domanska and J. A. Gonzalez, Fluid Phase Equilibr. 129, 139 (1997) (secondary reference).

322. S. Miskiewicz, K. Rieser, and T. Dorfmuller, Ber. Bunsenges. Phys. Chem. 80, 395 (1976).

323. H. D. Burrows, M. da Graca M. Miguel, A. P. Varela, and R. S. Becker, Thermochim. Acta 279, 77 (1996).

324. V. M. Garcia, M. I. Redondo, M. V. Redondo, and M. V. Roux, J. Chem. Soc., Faraday Trans. 93, 2509 (1997).
325. M. Siniti, J. Carre, J. M. Letoffe, J. P. Bastide, and P. Claudy, Thermochim. Acta 224, 97 (1993).

326. B. V. Lebedev, N. N. Smirnova, V. G. Yasil'ev, E. G. Kiparisova, and V. I. Kleiner, Vysokomolekulyarnye Soedineniya Ser. A 36, 1413

(1994).

327. J. W. McCargar and W. E. Acree, Jr. (unpublished data).

328. J. C. M. Li, J. Am. Chem. Soc. 78, 1081 (1956).

329. J. M. Rosen, Microscope (London) 17, 141 (1969).

330. G. Bertholon and R. Perrin, Bull. Soc. Chim. France 7/8, 1537 (1975)

331. I. M. Jalal, G. Zografi, A. K. Rakshit, and F. D. Gunston, Chem. Phys. Lipids 31, 395 (1982).

332. J. Zhang, R. Hu, C. Zhu, G. Feng, and Q. Long, Thermochim. Acta 298, 31 (1997).

333. R. W. Crowe and C. P. Smyth, J. Am. Chem. Soc. 72, 1098 (1950). 334. T. Kubota, J. Chem. Soc. Jpn. 53, 404 (1932).

335. L. L. Burger and G. H. Cady, J. Am. Chem. Soc. 73, 4243 (1951). 336. F. P. Schwarz, J. Solution Chem. 25, 471 (1996).

337. B. V. Lebedev, T. A. Bykova, N. V. Novoselova, N. G. Senchenya, and K. A. Mager, Polym. Sci. USSR, Ser. A 37, 886 (1995).

338. B. V. Lebedev, T. A. Bykova, E. G. Kiparisova, B. G. Velen'kaya, and V. N. Filatova, Polym. Sci. USSR, Ser. A 37, 126 (1995). 339. N. N. Smirnova, B. V. Lebedev, E. G. Kiparisova, K. L. Makovetskii, and I. Y. Ostrovskay, Polym. Sci. USSR, Ser. A 39, 893 (1997).

340. A. Cingolani and G. Berchiesi, J. Therm. Anal. 6, 87 (1974). 341. R. J. L. Andon, J. F. Counsell, and J. F. Martin, J. Chem. Soc. A 1894 (1968)

342. E. M. D. Keegstra, V. van der Mieden, J. W. Zwikker, L. W. Jenneskens, A. Schouten, H. Kooijman, N. Veldman, and A. L. Spek, Chem. Mater. 8, 1092 (1996).

343. J. N. Hay, J. Polym. Sci. Part B 8(6), 395 (1970).

344. G. M. Stack, L. Mandelkern, C. Krohnke, and G. Wegner,

Macromolecules 22, 4351 (1989).

345. C. J. Bowden, T. M. Herrington, A. M. Moseley, and R. Richardson, Liq. Cryst. 6, 825 (1995).

346. F. Hentrich, C. Tschierske, and H. Zaschke, Ang. Chem. Int. Ed. Eng. 30, 440 (1991).

347. F. Hentrich, S. Diele, and C. Tschierske, Liq. Cryst. 17, 827 (1994). 348. J. I. Macnab and J. A. Joy, Thermochim. Acta 259, 31 (1995).

349. N. N. Smirnova, B. V. Lebedev, E. G. Kiparisova, K. L.

Makovetskii, and L. I. Gorbacheva, Polym. Sci. USSR 34, 72 (1992).

350. T. A. Bykova, Y. G. Kiparisova, B. V. Lebedev, K. A. Mager, and Y. G. Gololobov, Polym. Sci. USSR 33, 537 (1991).

351. T. A. Bykova, E. G. Kiparisova, B. V. Lebedev, N. G. Senchenya, K. A. Mager, and Y. G. Gololobov, Polym. Sci. USSR 35, 8 (1993).

352. J. M. Rosen, J. R. Holden, and H. T. Simmons, Mol. Cryst. Liq. Cryst. 6, 205 (1969).

353. T. G. Kulagina and B. V. Lebedev, Russ. J. Phys. Chem. 71, 520 (1997).

354. R. Puliti, C. De Sena, and C. Giancola, J. Thermal Anal. 48, 1249 (1997).

355. R. Puliti, G. Barone, C. Giancola, and C. A. Mattia, J. Mol. Struct. 382, 197 (1996).

356. G. Barone, C. Giancola, T. H. Lilley, C. A. Mattia, and R. Puliti, J. Thermal Anal. 38, 2771 (1992).

357. D. J. Grant and I. K. A. Abougela, Int. J. Pharm. 17, 77 (1983).

358. A. Martin and M. J. Miralles, J. Pharm. Sci. 71, 439 (1982).

359. S. A. Curran and J. Asrar, Mol. Cryst. Liq. Cryst. 148, 255 (1987). 360. J. T. S. Andrews and E. F. Westrum, J. Phys. Chem. 74, 2170 (1970).

361. J. Lohmann, R. Joh, and J. Gmehling, J. Chem. Eng. Data 42, 1170 (1997).

362. W. V. Steele, R. D. Chirico, S. E. Knipmeyer, and A. Nguyen, J. Chem. Eng. Data 42, 1021 (1997).

363. W. V. Steele, R. D. Chirico, A. B. Cowell, S. E. Knipmeyer, and A. Nguyen, J. Chem. Eng. Data 42, 1053 (1997). 
Table 8. References to Tables 5, 6, and 7-Continued

364. V. V. Kafarov, I. N. Dorokhov, V. N. Vetokhin, and L. P. Volkov, Dokl. Akad. Nauk SSSR 298, 641 (1987).

365. J. Sciesinski, J. Mayer, T. Wasiutynski, E. Sciesinska, and J. Wojtowicz, Phase Trans. 54, 15 (1995).

366. A. Terol, B. Pauvert, A. Bouassab, P. Chevallet, and G. Cassanas, Thermochim. Acta 211, 279 (1992).

367. D. J. Wren and A. C. Vikis, J. Chem. Thermodyn. 14, 455 (1982).

368. T. G. Kulagina and B. V. Lebedev, Russ. J. Phys. Chem. 71, 709 (1997).

369. B. V. Lebedev, T. G. Kulagina, A. A. Cheremukhina, and E. N. Karataev, Russ. J. Gen. Chem. 66, 880 (1996).

370. S. J. Price and M. G. Jacko, Can. J. Chem. 43, 3481 (1965).

371. H. Nakazuma, T. Kitao, and K. Ohnishi, J. Heterocyclic Chem. 25, 1427 (1988).

372. A. Tero, A. Boussab, B. Pauvert, P. Chevallet, and G. Cassanas, Thermochim. Acta 225, 97 (1993).

373. J. Nurit, J. M. Leloup, B. Pauvert, O. Doumbia, P. Chevallet, and A. Terol, J. Thermal Anal. 45, 535 (1995).

374. L. Yang, H. Yin, W. Zhu, and S. Niu, J. Thermal Anal. 45, 207 (1995).

375. L. Abate, B. Palecz, C. Giancola, and G. Della Gatta, J. Chem.

Thermodyn. 29, 359 (1997).

376. F. Shafizadeh, G. D. McGinnis, R. A. Susott, and C. W. Philpot, Carbohydrate Res. 15, 165 (1970).

377. G. J. Kabo, A. V. Blokhin, A. A. Kozyro, and V. V. Diky,

Thermochim. Acta 290, 13 (1996).

378. F. P. Schwarz, J. Sol. Chem. 25, 471(1996).

379. M. Kaminski and W. J. Zielenkiewicz, J. Chem. Thermodyn. 28, 153 (1996).

380. L. S. Santos, Jr., S. Roca, and C. Airoldi, J. Chem. Thermodyn. 29, 661 (1997)

381. M. R. Smith, K. J. Irgolic, E. A. Meyers, and R. A. Zingaro,

Thermochim. Acta 1, 51 (1970).

382. C. Sunwoo and H. Eisen, J. Pharm Sci. 60, 238 (1971).
383. J.-L. M. Abboud, P. Jimenez, M. V. Roux, C. Turrion, C. Lopez-Mardomingo, A. Podosenin, D. W. Rogers, and J. F. Liebman, J. Phys. Org. Chem. 8, 15 (1995).

384. F. P. Schwarz, J. Sol. Chem. 25, 471 (1996).

385. S. P. Verevkin, B. Dogan, J. Hadrich, H.-D. Beckhaus, and C. Rüchardt, J. Prakt. Chem. 337, 93 (1995).

386. Z. Yang, G.-E. Yu, J. Cooke, Z. Ali-Adib, K. Viras, H. Matsuura, A. J. Ryan, and C. Booth, J. Chem. Soc., Faraday Trans. 92, 3173 (1997).

387. L. A. Torres, R. Gudino, I. Hernandez-Contreras, M. E. Ochoa, N. Farfan, and R. L. Santillan, J. Chem. Thermodyn. 27, 779 (1995). 388. G. J. Davis, R. S. Porter, and E. M. Barrall, Mol. Cryst. Liq. Cryst. 10, 1 (1970).

389. B. Wunderlich, Thermal Analysis (Academic, New York, 1990), Chap. 5, ATHAS Appendix, pp 417-431.

390. D. W. Van Krevelen, in Computational Modeling of Polymers, edited by J. Bicerano (Marcel Dekker, New York, 1992), part I, Group Contribution Techniques, pp. 55-123.

391. A. M. King and W. E. Garner, J. Chem. Soc. 1372-76 (1936).

392. P. Mura, G. P. Bettinetti, M. T. Faucci, and P. L. Parrini,

Thermochim. Acta 321, 59 (1998).

393. A. Gonthier-Vassal and H. Szwarc, Thermochim. Acta 320, 141 (1998).

394. S. H. Neau, S. V. Bhandarkar, and E. W. Hellmuth, Pharm Res. 14, 601 (1997).

395. A. Ohm and B. C. Lippold, Int. J. Pharm. Tech. Prod. Mfg. 6, 1 (1985)

396. K. C. James and M. Roberts, J. Pharm. Pharmac. 20, 709 (1968).

397. S. Verevkin, J. Morgenthaler, and C. Ruechardt, J. Chem.

Thermodyn. 29, 1175 (1997).

398. U. Domanska, Polish J. Chem. 72, 925 (1998).

399. O. A. Rajewskii, B. P. Solowiew, and L. W. Goworkowa, Zh. Ob. Khim. 55, 1381 (1985).

\section{Acknowledgments}

The Research Board of the University of Missouri and the USEPA are gratefully acknowledged for support of portions of this work.

\section{References}

${ }^{1}$ J. S. Chickos, R. Annunziata, L. H. Ladon, A. S. Hyman, and J. F. Liebman, J. Org. Chem. 51, 4311 (1986).

${ }^{2}$ J. S. Chickos, D. G. Hesse, S. Y. Panshin, D. W. Rogers, M. Saunders, P. M. Uffer, and J. F. Liebman, J. Org. Chem. 57, 1897 (1992).

${ }^{3}$ J. S. Chickos, D. G. Hesse, S. Hosseini, J. F. Liebman, G. D. Mendenhall, S. P. Verevkin, K. Rakus, H.-D. Beckhaus, and C. Rüchardt, J. Chem. Thermodyn. 27, 693 (1995).

${ }^{4}$ E. M. Barral II and J. F. Johnson, in Liquid Crystals and Plastic Crystals, edited by G. W. Gray (1974), Vol. 2.

${ }^{5}$ P. Walden, Z. Elektrochem. 14, 713 (1908).

${ }^{6}$ R. M. Dannenfelser, N. Surendren, and S. H. Yalkowsky, SAR QSAR Environ. Res. 1, 273 (1994).

${ }^{7}$ R. M. Dannenfelser and S. H. Yalkowsky, Ind. Eng. Chem Res. 35, 1483 (1996).

${ }^{8}$ J. S. Chickos, D. G. Hesse, and J. F. Liebman, J. Org. Chem. 55, 3833 (1990).
${ }^{9}$ J. S. Chickos, C. M. Braton, D. G. Hesse, and J. F. Liebman, J. Org. Chem. 56, 927 (1991)

${ }^{10}$ J. S. Chickos, W. Acree, Jr., and J. F. Liebman, in Computational Thermochemistry: Prediction and Estimation of Molecular Thermodynamics, edited by K. K. Irikura and D. J. Frurip, ACS Symp. Ser. 677 (ACS: Washington DC, 1998); pp. 63-91.

${ }^{11}$ E. S. Domalski and E. D. Hearing, J. Phys. Chem. Ref. Data 25, 1 (1996).

${ }^{12}$ S. L. Björnstad and G. Borgen, Acta Chem. Scand 29B, 13 (1975).

${ }^{13}$ J. A. R. Cheda and E. F. Westrum, Jr., J. Phys Chem. 98, 2482 (1994).

${ }^{14}$ W. E. Acree, Jr., Thermochim. Acta 189, 37 (1991).

${ }^{15}$ B. F. Rordorf, Proceedings of the 5th International Symposium on Chlorinated Dioxins and Related Compounds, Bayreuth, Germany, September 16-19 [Chemosphere 15, 1325 (1986)].

${ }^{16}$ W. Zielenkiewicz, A. Zielenkiewicz, and K. L. Wierzchowski, J. Sol. Chem. 23, 1125 (1994).

${ }^{17}$ A. Regosz, A. Chmielewska, T. Pelpinska, and P. Kowalski, Pharmazie 49, 371 (1994).

${ }^{18}$ J. R. Donnelly, L. A. Drewes, R. L. Johnson, W. D. Munslow, K. K. Knapp, and G. W. Sovocol, Thermochim. Acta 167, 155 (1990).

${ }^{19}$ J. S. Chickos and M. J. E. Sternberg, Thermochim. Acta 264, 13 (1995).

${ }^{20}$ S. D. Roy and G. L. Flynn, Pharm. Res. 5, 580 (1988).

${ }^{21}$ L. Yang, H. Yin, W. Zhu, and S. J. Niu, Thermal Anal. 45, 207 (1995).

${ }^{22}$ L. Borka and J. K. Haleblian, Acta Pharm. Jugosl. 40, 71 (1990). 